\title{
Resilience in Children in the Aftermath of Disasters: A Systematic Review and a New Perspective on Individual, Interpersonal, Group, and Intergroup Level Factors
}

\begin{abstract}
Alessia Cadamuro $^{\mathrm{a}}$, Michèle D. Birtel ${ }^{\mathrm{b}}$, Gian Antonio Di Bernardo ${ }^{\mathrm{c}}$, Eleonora Crapolicchio ${ }^{\mathrm{d}}$, Loris Vezzalie, John Drury ${ }^{\mathrm{f}}$
\end{abstract}

${ }^{a}$ University of Modena and Reggio Emilia, Department of Biomedical, Metabolic and Neural

\section{Science}

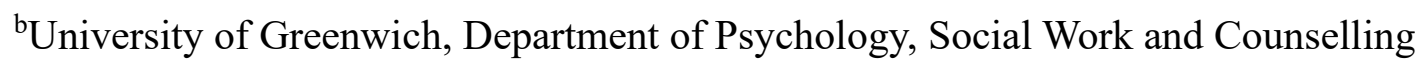

${ }^{c}$ University of Modena and Reggio Emilia, Department of Education and Humanities

${ }^{\mathrm{d}}$ University of Sacred Heart of Milan, Department of Psychology

eUniversity of Modena and Reggio Emilia, Faculty of Medicine

fUniversity of Sussex, School of Psychology

Author Note

Correspondence concerning this article should be addressed to Alessia Cadamuro, University of Modena and Reggio Emilia, viale Allegri 9, 42121, Reggio Emilia, Italy. Email: alessia.cadamuro@unimore.it. 


\title{
Resilience in Children in the Aftermath of Disasters: A Systematic Review and a New Perspective on Individual, Interpersonal, Group, and Intergroup Level Factors
}

\begin{abstract}
Disasters can impact upon individuals, families, and communities in multiple ways. Research has mainly focused on risk and protective factors relating to the child (individual level) and the family (interpersonal level), not taking into account the processes at the level of social groups. The present review aims to (a) review psychological research on disasters determined by natural events in childhood, (b) distinguish individual, interpersonal, group, and intergroup levels, (c) emphasize the importance of considering resilience as a key outcome.

We reviewed 294 studies (in addition to 28 reviews-meta-analyses, and 29 naturalistic interventions), and identified factors at the individual (e.g., demographics, exposure, individual differences), interpersonal (e.g., parent-child relationship, family and school environment), group (e.g., social identity, group membership), and intergroup (relations between different groups) levels. We argue that an integrated model of these factors and their interplay is needed to design interventions to enhance resilience in children and their communities. We extend previous theorizations by providing a wider conceptualization of distress and resilience, and by considering the interplay between factors at different levels. A multidimensional approach to the consequences of disasters in children is crucial to understand their development and well-being, and to design effective interventions.
\end{abstract}

Keywords: disasters; children; PTSD; social identity; resilience; distress 
Disasters, which occur almost every day all over the world, can take many forms such as earthquakes, floods, or tsunamis. They affect many people simultaneously and cause detrimental psychological consequences such as distress, behavioral problems and, for a minority, trauma (Masten \& Osofsky, 2010; Wolmer, Laor, \& Yazgan, 2003). The impact of a disaster cannot be defined by the magnitude of the disruption alone: its consequences are dependent upon the interplay of the hazard and the context (social, economic and political processes) which define the vulnerability of a community (Tanner \& Seballos, 2012).

It is estimated that $11 \%$ of individuals have been exposed to a disaster before 16 years of age (Copeland, Keeler, Angold, \& Costello, 2007). This is particularly worrying, because children may find threatening events especially distressing due to the challenge of dealing with rapid emotional and psychological development, fewer coping resources, and high dependence on caregivers for protection (Baggerly \& Exum, 2008; De Young, Kenardy, \& Cobham, 2011).

Many psychiatric and somatic symptoms have been reported in children following traumatic events (Drury, Scheeringa, \& Zeanah, 2008; Farooqui et al., 2017), including the development of post-traumatic stress disorder (PTSD) symptoms (Bokszczanin, 2007), posttraumatic stress symptoms (PTSS) (Furr, Comer, Edmunds, \& Kendall, 2010), depressive reactions (La Greca, Silverman, \& Wasserstein, 1998; Tang, Liu, Liu, Xue C., \& Zhang, 2014), separation and generalized anxiety (Costa, Weems, \& Pina, 2009), externalizing behavior problems (De Young et al., 2011), and general impaired quality of life (e.g., Copeland et al, 2007). These symptoms may persist over time (La Greca, Silverman, Lai, \& Jaccard 2010; Lai, La Greca, Auslander, \& Short, 2013) and interfere with children's functioning in different domains (Samuelson, Krueger, Burnett, \& Wilson, 2009; Schoeman, Carey, \& Seedat, 2009). 
Children's vulnerabilities have been described as a function of interacting factors, such as age, gender, geographical location, exposure, family characteristics, ethnicity, socio-economic status, disability, health status, and social networks (Gordon-Hollingsworth, Yao, Chen, Qian, \& Chen, 2015; Peek, 2008). The ability of a child to react positively depends on internal factors, but also on how much the adult world is able to accompany them in the traumatic experience and the extent to which this experience is managed and elaborated by the adults themselves. For this reason, parental and, more in general, social support could be critical protective factors and work as moderators of negative outcomes in children exposed to distressing experiences (Cheng, Liang, Zhou, Eli, \& Liu, 2019; Danielson et al., 2016). We argue for the importance of identifying all the variables playing a role in children's reactions to disasters and specifically in resilience.

Typically, research on disasters has largely focused on the individual and, partly, on the interpersonal level, mostly focusing on factors relating to the child as an individual and their immediate social network, such as the family. However, what characterizes a disaster is its collective nature. Distress produced by disasters is distinct from distress experienced at an individual level, as it can also include factors such as discrimination against minority groups, shared and vicarious distress, and threats to the child's social identity, all of which can be fueled by the disaster and in turn exacerbate its effects. In other words, distress can also depend (at least in part) on factors related to individuals' group of belonging. As an example, highly prejudiced majority group children may be reluctant being categorized in a common group (e.g., the group of disaster survivors) including the discriminated minority outgroup, since it may threaten the need for intergroup distinctiveness (Jetten \& Spears, 2003). Again, observing the others' distress, 
therefore experiencing it vicariously, may add to own distress and produce a sense of helplessness which reduces the chance of recovering.

However, social identities can also act as buffers, or even as motivating factors leading to resilient responses in emergencies and disasters (Vezzali, Andrighetto, Drury, Di Bernardo, \& Cadamuro, 2017). In fact, while distress because of unique personal experiences may create barriers toward the others since it concerns the individual only, distress following a disaster can be perceived as a shared experience, fostering a common response and understanding. Distress and trauma are experienced collectively (Kaniasty \& Norris, 1999), and only by taking into account all the components that reciprocally interact in a community (and beyond) at the individual, interpersonal, group, and intergroup level, it is possible to understand how to build personal and collective psychosocial resilience.

Note that there is a substantial body of literature available from different disciplines that has focused on the impact of disasters, also focusing on vulnerable populations. This literature places importance on the child's socio-ecological context in determining the impact of disasters. Disasters impact upon mental and physical health in particular among children belonging to certain social groups, i.e., groups with certain demographics and social vulnerabilities such as ethnic minority status, gender, and disability (for an overview, see Peek, Abramson, Cox, Fothergill, \& Tobin, 2018). Children are embedded in a social ecological system consisting of microsystem (direct relations with the family, peers, schools, institutions), mesosystem (dyadic relations between two microsystems), exosystem (indirect relations in the social system which influence the child such as media or parents' workplace), macrosystem (culture the child lives in, such as ideologies and socioeconomic status), and chronosystem (changes over time such as parent's death or disasters) (Bronfenbrenner, 1979). These factors of person, context, time and 
processes interact with each other (Bronfenbrenner, 2005). In other words, existing literature from different fields recognizes the importance of factors that go beyond the individual, and highlights the relevance of the larger social context. In order to provide a systematization of these indications, our review considers studies demonstrating from a psychological point of view the impact of these different factors, differentiating them in levels (individual, interpersonal, group, and intergroup), and argues for the importance of considering their complex interplay (see Figure 1).

The present review has three aims. First, it provides a systematic review of psychological research on disasters in childhood, and specifically on disasters determined by natural events. Second, extending previous work which has mainly focused on individual-level outcomes, we distinguish between individual, interpersonal, group, and intergroup levels. We argue for an integrated model in which these levels interact in determining psychological outcomes. Third, in contrast to most previous research that has focused on negative outcomes, we argue that the final outcome of the model should be resilience. With this review, we hope to stimulate future research that examines disasters in their social context.

\section{The Present Review}

Computerized searches were conducted between 26 and 30 June 2020, by using the PsycINFO database, using the following keywords and their combinations: child*, natural disaster*, earthquake, flood, hurricane, tornado, tsunami. Studies were selected based on four inclusion criteria: (1) include children up to 12 years old in the sample (as childhood is defined to end at the age of 12; cf. Shaffer \& Kipp, 2009); (2) be in English, (3) concern disasters; (4) be quantitative (since we are interested in statistical effects of identified factors, in addition to their interactions; see Table 1 in online supplemental material - OSM). 
After initial article identification, the title and abstract of each article were read to determine if the article met inclusion criteria. In so doing, we generally referred to criteria provided by PRISMA guidelines for systematic reviews (Moher, Liberati, Tetzlaff, Altman, \& The PRISMA Group, 2009); see Figure 1 in OSM. Each study was coded by the first author.

The literature search yielded 2,055 records. After removing duplicates, books, editorial, letters, book reviews, work written in languages other than English, sample age over 12 years, $(n$ $=1020)$ articles were screened by title and abstract. Of these, $(n=572)$ were selected for full text review for relevance. The inconsistencies were discussed by the team of authors.

After removing articles not meeting inclusion criteria, 294 eligible articles published before $30^{\text {th }}$ June 2020 were included. Studies were coded according to several characteristics, including disaster type, number, type and age of participants, country, study design, level of analysis (individual, interpersonal, and group, intergroup), variable type (independent, mediator, moderator, dependent), time-points of assessment and dependent variables. Studies coded according to these characteristics are shown in Table 1 in OSM. In addition, in order to provide a fuller picture of literature of the field, we also included two additional tables in OSM of articles/studies not included in the 294 studies reported in Table 1 in OSM: in Table 2 in OSM we report 28 reviews-meta-analyses, in Table in OSM 3 we report 29 naturalistic interventions.

Finally, we include tables in OSM taking into account specific study characteristics: type of disaster (Table 4), frequencies of study design (Table 5), level (Table 6), independent (Table 7) and dependent (Table 8) variables, and finally the geographical distribution of the studies (Table 9).

From the analysis of the selected literature, it was possible to evaluate the number of studies divided by type of disaster. As can be seen in Table 4 in OSM, the majority of the studies 
examined children in the aftermath of earthquakes (126) followed by hurricanes (92) and tsunami (27), while few studies were related to cyclones, lightning-strikes, tornadoes, volcanoes and storms. These numbers could be explained by the frequency of these phenomena, or by the destructive impact that earthquakes and hurricanes have on a large part of the population (that makes them an especially interesting target of research). Given the characteristics of the territories, consistent with the type of disasters, the research was carried out mainly in the United States (41\%), followed by China (17\%), Turkey (6\%), Japan (6\%), Southeast Asia (5\%), and Australia (3\%) (Table 9 in OSM). It is also worth noting that most of the studies (62\%) employed a cross-sectional design (Table 5 in OSM). Importantly for our purposes, most studies focused on individual-level factors (59\%), whereas a lower number took into account interpersonal-level (37\%) and intergroup-level factors (2\%); a very small number of studies (3\%) included two or more levels (Table 6 in OSM). It is also interesting to note that, although there are various relevant reviews in the field (Table 2 in OSM), these had a more narrow focus, for instance referring to one type of disaster or focusing on one specific level (e.g., individual), resulting in a smaller number of studies reviewed, while our work provides a more comprehensive overview.

After presenting the outcome variables examined by research, we will review the individual, interpersonal, group, and intergroup level factors that impact upon children. Then, we will argue for the need of a new integrated perspective that takes into account how these factors interact and can lead to resilience.

\section{Consequences of Disasters for Children}

Overall, the dependent variables most frequently investigated concern children's psychological well-being (cf. Table 8 in OSM). As also acknowledged by other reviews (Furr et 
al., 2010), disasters cause psychiatric and somatic symptoms. Post-traumatic stress disorder (PTSD), one of the most studied outcomes (40\%), may persist for months or years. Other consequences include major depressive disorders (15\%), generalized anxiety disorders (8\%), adjustment disorders (7\%), post-traumatic stress symptoms (7\%); internalizing/externalizing problems (5\%), and different types of behavioral and emotional problems (4\%).

Disasters, in addition to impacting upon relations between individuals, can also inhibit family functioning and disrupt many other aspects of life, at the level of peer groups, schools, neighborhoods, communication systems, the economy, and international relations (Masten \& Obradovìc 2008; Terranova, Morris, Myer, Kithakye, \& Morris, 2015).

In sum, research has generally focused on consequences at the individual level, mostly related to health outcomes; less work in psychology has investigated consequences at the interpersonal and even less at the community level (including group and intergroup levels theorized in this review). Notably, these consequences were generally negative. Studies considering the group and intergroup levels sometimes find positive effects of disasters, such as increases in the desire to meet and help other survivors (Vezzali et al., 2017). We argue that future research should examine wider consequences of disasters, to investigate how they impact upon relations at the interpersonal, group, and intergroup level.

\section{Individual Level}

Research has mainly focused on individual factors as predictors of psychological outcomes, which we broadly distinguish in terms of demographic factors, level of exposure, coping, theory of mind and other individual differences (cf. Table 7 in OSM).

Demographic factors such as age and gender are amongst the most frequently investigated factors ( $45 \%$ and $47 \%$ respectively). Studies examining gender showed mixed 
results: while the majority of studies revealed greater stress and depression in girls than boys (Furr et al., 2010; Kronenberg et al. 2010; Silwal, Dybdahl, Chudal, Sourander, \& Lien, 2018), some found a prevalence in boys than girls (Eksi et al., 2007) and others did not reveal any gender effect (e.g., Catani, Jacob, Schauer, Kohila, \& Neuner, 2008; Tao, Duan, \& Shi, 2014).

Gender was also found to influence children's defensive style and coping, the availability and use of social support, and expectations for response and recovery, with girls generally reporting more self-blaming, depression and rationalization compared to boys (Han, Zhang, \& Zheng, 2012).

Studies examining age as a predictor of trauma symptoms also showed mixed results, with some studies finding no age differences (e.g., Hensley-Maloney \& Varela, 2009; Marsee, 2008; Weems et al., 2007), and others showing that older children display greater PTS than younger children (e.g., Saylor, Cowart, Lipovsky, Jackson, \& Finch, 2003). However, most of the studies revealed that younger children are more at risk than older children for developing PTSD symptoms (Kronenberg et al., 2010; Osofsky, Osofsky, Kronenberg, Brennan, \& Hansel, 2009).

Other demographic factors are related to socioeconomic status and living conditions, with individuals with lower socioeconomic status or worse living conditions more likely to develop distress (Kar et al., 2007).

Table 1 in OSM suggests that differences in context or type of disaster are not sufficient to account for inconsistencies in effects found for age and gender. Other individual variables such as levels of exposure or previous emotional difficulties, or interpersonal variables such as social support (see next section), may interact with these variables and allow a clearer understanding of the direction of the effects. 
An individual predictor considered by the majority of studies (57\%) is the level of exposure to the disaster, including objective and/or subjective elements of the child's disaster experience (Tang, $\mathrm{Xu}, \mathrm{Li}, \mathrm{Lu}, \& \mathrm{Xu}, 2018$ ). Regarding objective elements, only a few studies ( $9 \%$ of all the studies considered) found that proximity to the disaster was associated with distress (Catani et al., 2010; Feo et al., 2014), while others have found limited effect for this variable (Evans, \& Oehler-Stinnett, 2006). Furr and colleagues (2010) found a small to medium effect of disaster exposure on PTS in children and youth, with greater effects observed for disasters characterized by greater loss of life, perceived threat, and loss of a loved one (Usami et al., 2019). Felix et al. (2011) found no difference in PTSD rates between exposed and nonexposed samples in the aftermath of a disaster; in contrast, exposed children revealed greater major depression, social phobia, and separation anxiety.

Note that perceptions of exposure can be critically dependent on other variables, like age. For instance, older children experienced greater adversity, possibly because of greater awareness of what is happening, greater mobility, higher direct exposure to community effects and media exposure (Masten \& Osofsky, 2010).

It is also possible that child proximity offers only a simplistic measure of exposure, and what matters more at the psychological level is the perception of the disaster impact (e.g., Pfefferbaum et al., 2000). Consistently, there is considerable evidence that subjective exposure to the disaster and related elements (e.g., perceived life threat) systematically impact on healthrelated outcomes (La Greca et al., 2010) and can be more relevant in determining children's post-disaster response (Silverman \& La Greca, 2002).

Another individual-level variable, relevant to understand how people respond to the disaster, is coping style (8\%). The effects of coping with the consequences of disasters differ 
depending on the type of coping children were able to adopt, with negative coping (venting negative feelings, ruminating, and avoiding stressors) generally showing negative, and active coping (cognitive restructuring, emotional regulation, social support) showing positive effects on health-related, but also on cognitive (e.g., cognitive performance) variables (Cadamuro, Versari, Vezzali, Giovannini, \& Trifiletti, 2015; Terranova, Boxer, \& Morris, 2009).

Individual differences in Theory of Mind (ToM), mental health and cognitive skills also play a relevant role. ToM, that is the ability to understand others' mental states and to understand and share emotions with others, appears to buffer the negative outcomes of disasters. For example, ToM mediated the effect of coping style and was associated with better cognitive performance (Cadamuro et al., 2015).

Sprung (2008) reported that 5- to 8-year-old children who had more developed ToM skills displayed stronger intrusive thoughts after Hurricane Katrina, but were also more receptive to learning strategies to cope with them. Greater competency beliefs promoted more posttraumatic growth in children aged 6 to 15 who were exposed to Hurricane Floyd (Cryder, Kilmer, Tedeschi, \& Calhoun, 2006).

The level of anxiety or the tendency to experience negative emotions are risk factors that have been linked to the development of PTSD. Pre-existing child anxiety and depression have been shown to significantly predict post-disaster PTSD (Weems et al., 2007). Furthermore, cognitive (e.g., general intelligence, cognitive flexibility) and self-regulation skills are protective factors for children in disasters (Masten \& Obradovì 2008). For example, Terranova et al. (2009) reported that effortful control abilities (shifting, planning, and inhibiting unwanted behaviors) buffered children's PTSD symptoms after Hurricane Katrina. 
Importantly, the above individual factors may interact with other factors to ultimately determine the impact of disasters. For instance, children from minority groups (e.g., with disabilities) or living in poverty may be more exposed to negative consequences at physical, psychological, and educational level that may aggravate the impact of disasters (Peek \& Stough, 2010; Takada, 2013). Therefore, it is important to also consider factors relating to the social context in which children live. Children live within a family system (interpersonal level) that is integrated into a community (group level) within a cultural and ethnic context (intergroup level).

\section{Interpersonal Level}

Several factors regarding relations with relevant other individuals contribute to determining children's responses to disasters, such as parents (parents' reactions, parental coping, relations between parents), social (school) environment, and social support (Table 7 in OSM).

The first important determinants of child's adjustment in disaster settings are the parents, in particular the quality of interactions within a family and the family's reactions (Hausman, et al., 2020; Hlodversdottir, et al., 2018; Kessel et al., 2019). Osofsky and colleagues (2009) reported higher levels of post-traumatic stress symptoms among young children who had been separated from caregivers during the hurricane. Children and their parents respond to each other's stress, and parents serve as role models for coping, therefore different studies (15\%) have examined adaptation to disasters on a family level. Parents' reactions to the disaster and their ability to make the child feel secure are critical factors impacting upon the child (Mikyung, Sehwa, \& Lee-jin, 2020) Especially, children's emotional and behavioral problems and their distress are associated with irritable, depressed, distressed, dysfunctional, and overprotective families (Cadamuro, Versari, Vezzali, \& Trifiletti, 2016). Parents and caregivers with such 
characteristics may therefore be unable to provide adequate support as they face their own challenges in dealing with the effects of potentially traumatic events (Felix, Kaniasty, You, \& Canino, 2015). Finally, parental conflict has deleterious effects on children's responses.

Wasserstein and La Greca (1998) found that higher parental conflict after Hurricane Andrew was associated with more stress symptoms among children, possibly because it lowered reciprocal help among family members.

Health and psychological effects on children can manifest even years later, starting from prenatal exposure to disaster. Research has demonstrated associations between various types of prenatal maternal objective exposure (e.g., financial loss) or psychological distress (anxiety, depression, psychological distress, stress), and children's anxiety symptomatology (internalizing behaviors, anxiety symptoms) and cognitive development (intellectual, language and functional play) (Buthmann et al., 2019; Li et al, 2019; Nomura et al., 2019; Simcock et al., 2019; Strahm et al., 2020).

Among the protective factors for children offered by communities in the aftermath of disaster are schools and other safe environments for children to play and learn (Le Brocque, et al., 2017). Schools can provide a stable, supportive environment for children and a sense of safety and security (Mooney, Tarrant, Paton, Johnston, \& Johal, 2020). These institutions serve to re-establish routines in a child's life, and may provide respite for parents, opportunities for peer interaction, constructive activities, and connections to relevant adults (e.g., teachers) or peers. Interventions realized in the schools have helped children to articulate their feelings, process grief, regulate emotions (Powell \& Holleran-Steiker, 2017), improve coping skills and peer relationships (Amin, Nadeem, Iqbal, Asadullah, \& Hussain, 2020; Hansel, Osofsky, Osofsky, \& Speier, 2019). 
Disasters tend to damage children's social networks (La Greca et al., 2010). It is not surprising then that social support ${ }^{1}$ has been the main protective factor identified by research (12\%), being associated with positive outcomes for health and well-being (Kroska, Miller, Roche, Kroska, \& O'Hara, 2018; Usami et al., 2019) ) (cf. Table 8 in OSM). Further indirect evidence for the role of social support on health and well-being comes from studies examining the effects of social exclusion. Being excluded or rejected by other people (especially in threatening situations like after a disaster) can lead to lower self-esteem, loss of control, anxiety, depression, and neurological responses associated with physical pain (Shelton, Richeson, \& Vorauer, 2006). Social support and self-esteem are closely linked and can be intended as coping mechanisms in stressful situations (Taylor \& Stanton, 2007).

\section{Group and Intergroup Level}

Only recently has psychological research on disasters started taking into account the processes involved when focusing on the individual as a member of social groups, shifting the focus from one-to-one interactions (interpersonal level) to group processes and intergroup relations. Social support can be provided not only in interpersonal interactions (e.g., by an individual teacher), but also in groups (e.g., peer groups), as well as by communities composed of different social groups. Specifically, we consider social support given to individuals as members of an ingroup category, to be different from that given to outgroup members, which in turn is different from social support provided to people as unique individuals (i.e., when a social category is not salient). For instance, a child distressed because of the disaster can be helped cope with it individually by a psychologist. In contrast, in order to foster peer support as a means to cope with collective distress after a disaster, the school could organize interventions at the level of the class, explaining the importance of staying together and helping each other to face 
the disaster consequences. When however ingroup-outgroup distinctions are salient, especially when conflictual intergroup relations are considered, helping directed at the outgroup (even if the ingroup received the same help) may be perceived as a threat (since one may fear that the ingroup receives less help than it deserves), unless groups are included in a superordinate identity (Gaertner \& Dovidio, 2000). However, this research is almost non-existent when children are considered. We argue that processes at group and intergroup levels can strongly impact upon children, and need to be included in disaster research.

Note that although group and intergroup level can be distinguished, they are conceptually related (and this is why we considered both of them in the same section). The boundary between group and intergroup level may however be subtle. As we argued, for instance, the disaster can lead to a superordinate social identity as a community (group level), that in some cases re-shape relations between former relevant group identities (e.g., multicultural context - intergroup level) by making salient that they belong to a single group (if such a category already exists) or by creating a new emergent group.

In most studies taking into account the role of 'race', researchers investigated the differential impact of the event depending on one's ethnic group, with mixed findings. March, Amaya-Jackson, Terry, and Constanzo (1997) showed that race represents a risk factor for distress: African (both male and female) and Caucasian (female) Americans were more likely to exhibit post-traumatic stress and comorbid symptoms than Caucasians (males). Similarly, following Hurricane Hugo (Shannon, Lonigan, Finch, \& Taylor, 1994) and Hurricane Floyd (Pullins, McCammon, Lamson, Wuensch, \& Mega, 2005), African American youths reported greater PTSD symptoms than Caucasian children. These findings suggest that children from 
ethnic minority groups may have greater difficulty in responding to and recovering from the impact of the disaster.

By contrast, Jones, Frary, Cunningham,Weddle, and Kaiser (2001) assessed the impact of Hurricane Andrew on 212 African American, Caucasian, and Hispanic elementary and middle school children 6 months after the disaster, reporting no race differences. Similarly, Russoniello et al. (2002) found that ethnicity was not significantly associated with PTSD in a sample of 218 children aged 9-12 years after Hurricane Floyd.

Brown, Mistry, and Bigler (2007) showed that group-level factors may also impact on attributions. They found that Hurricane Katrina raised several issues related to race and class: older African American children were less likely than younger African American children to attribute the delayed relief to individual culpability, and slightly more likely to attribute it to racial discrimination. All youth believed in the role of the government, but younger children were more likely to rate it as effective.

The studies reported above considered group-level factors such as race mainly as a demographic variable, testing differences in distress between groups. Other studies instead focused on the different psychological processes that members of different groups can activate in response to disasters. When considered in these terms, group and intergroup processes can both be risk and protective factors for children in the context of disasters. On the one hand, disasters can exacerbate the detrimental consequences of intergroup processes. According to social identity theory (Tajfel \& Turner, 1979), we seek positive distinctiveness for our social identities, and one of the ways that we might positively differentiate our ingroup from an outgroup under certain conditions is by discrimination against that outgroup. For instance, the risk of discriminating against or excluding the minority group may be particularly relevant when 
resources are (perceived as) limited and need to be shared within other groups (Andrighetto, Vezzali, Bergamini, Nadi, \& Giovannini, 2016). When intergroup conflict is salient, the perception of limited resources may result in increased competition between groups and lower intentions to help the outgroup; or outgroup behavior can be interpreted negatively, and this negative contact may dampen the desire to support outgroup survivors of one's community (Vezzali et al., 2017).

Furthermore, in the long run categorization may cause segregation and conflict, weakening the community collective response (Andrighetto et al., 2016). In a study with elementary school children in affected areas six months after the 2012 Italian earthquake, categorizing as two groups was associated with Italian children's more negative outgroup attitudes toward immigrants (see Vezzali, Cadamuro, Versari, Giovannini, \& Trifiletti, 2015). In addition, different groups have different group norms, which may include hostility and negative stereotypes towards outgroups. When group identities and their negative norms are salient, this would lead to worse intergroup relations and to fewer resilient responses across the community as a whole.

On the other hand, social identities can act as buffers, or even as motivating factors leading to greater resilient responses, such as support and cooperation (Vezzali et al., 2017). Identifying as a member of a group enhances social cohesion and fosters helping among ingroup members (Turner et al., 1987). In a community characterized by several groups (e.g., multiethnic community), individuals can react to disaster threat by categorizing in terms of a specific subgroup (e.g., ethnic group) (Andrighetto et al., 2016). This categorization, that uses group identification as a buffer from threats from other groups, may be beneficial in terms of health outcomes (Shelton et al., 2006). This conclusion is consistent with the social cure approach, 
stating that social identities have a positive impact on post-disaster well-being (Jetten, Haslam, \& Haslam, 2015). Individuals are better able to cope with stressors if they embrace their group identity and if valued social identities can be maintained or new social identities can be developed (Jetten, Haslam, Haslam, \& Branscombe, 2009). The development of new positive and meaningful social identities can be a source of resilience in the face of trauma, and the reaction to trauma is worse if the trauma undermines relevant social identities (Muldoon et al., 2019). However, an important feature of disasters is that they can transform relations between people, including group boundaries. For instance, according to the concept of 'altruism born of suffering', the shared traumatic experience can forge new or reinforce existing relations between individuals (Staub, 2003). In social identity terms, new inclusive shared social identities can develop in disasters as a function of common fate (Drury, 2018). In line with altruism born of suffering (Staub, 2003), self-categorization can be at the superordinate level, including all members of the community irrespective of subgroups. Such identification enables benefits (in particular social support) previously reserved for the ingroup to be extended to outgroup members. In addition to fostering adaptive responses and shielding from negative outcomes typically associated with conflictual intergroup relations, superordinate identification can allow the creation of new or reinforcement of existing ties between individuals and a stronger community response, facilitating collective recovery. Consistent with this argument, among Italian children, higher perceived disaster threat was associated with more positive attitudes toward immigrants, and greater desire to have contact with and help them, via perceptions of belonging to the common group of Italian and immigrant child survivors (see Vezzali et al., 2017). 
Interestingly, even when not explicitly framed in terms of intergroup relations, referring to a shared identity can help strengthening community ties. Vezzali et al. $(2015,2016,2017)$ found that PTSD symptoms, used as independent variables as a form of subjective disaster exposure, were associated with greater identity fusion with other children exposed to the disaster, and in turn with greater perceptions that children survivors were members of a common group. These common group perceptions were associated with greater prosocial intentions toward other disaster survivors.

Depending on the salience of contextual social identities, responses to the disaster may differ. In some cases (intergroup level), formation of new identities may depend at least in part by recategorization of former groups; in other cases (group level), where other group identities are not salient and/or irrelevant, the only meaningful identity can be that of the community that has been struck by the disaster. In both cases, we argue that what is relevant is taking into account the new group identities stemming from the disaster that can be a strong determinant of attitudes and behaviors within, but also outside the community.

\section{Interplay between individual, interpersonal, group, and intergroup levels}

We have reviewed evidence that individual, interpersonal, group and intergroup variables can act as risk and protective factors in children in disasters. We propose that the impact of disasters on children is the result of a complex interplay between these levels (Figure 1). Each of them can buffer or enhance the direct (e.g., PTSD) and indirect (e.g., secondary stressors such as discrimination stemming from increased threat perceptions) consequences of the disaster and enhance (or undermine) resilience.

A likely possibility is that the four levels have additive effects, as also shown by studies testing these factors as independent variables within the same study (Felix, You, \& Canino, 
2013; La Greca et al., 2010) (cf. Table 7 in OSM). However, we argue that a full understanding of the impact of disasters can be understood when taking into account their reciprocal interactions. The aim of this section is to show evidence that factors pertaining to the four dimensions can interact in predicting outcome related to disasters.

With respect to interactions between individual and interpersonal dimensions, for instance, Cadamuro et al. (2015) found that whereas active coping (individual level) was positively associated with ToM abilities (which in turn were associated with better cognitive performance), the association between avoidant coping and ToM depended on perceived social support (interpersonal level), such that avoidant coping was positively associated with ToM only when perceived social support was high.

Furthermore, in a sample of elementary school children and their parents in the aftermath of an earthquake, Cadamuro et al. (2016) found that the indirect association of mothers' PTSD symptoms (interpersonal level) with increased reliance on negative coping (via children's PTSD symptoms), was buffered by ToM (individual level), i.e. this association was significant only when ToM was low. Felix et al. (2015) have shown that parent-child relationship quality (interpersonal level) acted as moderator of the influence of hurricane exposure (individual level) on physical health among children: hurricane exposure had weaker negative consequences when parent-child relationship quality was high.

Looking at characteristics of the school and community microsystem that may affect outcomes in children, Felix, You, and Canino (2013) found that gender (female) and living in poverty, as well as disaster exposure (all individual-level factors) were associated with an increased risk of internalizing psychopathology in children, but these effects were reduced when neighborhood climate was positive and school violence was low (interpersonal level). 
In relation to the interaction between individual and group levels, there is no systematic evidence, but we can suggest the process based on the broader literature on disasters. Thus those individuals who identify most strongly with other survivors of an earthquake have been found to be those most likely to be influenced by the sight of supportive behavior to be supportive themselves (see Drury, 2018; Drury et al., 2019).

Regarding the relation between interpersonal and intergroup level, it was found for instance that ethnic group membership (intergroup level) moderated the relation between parental conflict (interpersonal level) and PTSD following a hurricane, with detrimental effects of parental conflict significant for Hispanics, but not for Whites (Wasserstein \& La Greca, 1998).

With respect to individual-intergroup interplay, Vezzali et al.'s (2017) results showed that perceived disaster threat (individual level) was indirectly associated with greater contact and helping behavioral intentions toward the outgroup via greater perceptions of belonging to a common group among majority (Italian) but not minority (immigrant) children (moderation by ethnicity: intergroup level).

The scarcity (3\%) of studies testing interaction effects between factors related to the different levels in reviewed literature limit our possibility to make predictions about the direction of these interactions, also considering the rather large number of potential factors pertaining to each of these levels. However, from this analysis, it is clear that the different dimensions (individual, interpersonal, group, intergroup) can and do interact; therefore, considering them in isolation, only focusing on their additive effects, may be misleading and limit our capacity to fully understand the impact of disasters in children and how to react to it. In contrast, considering their interplay can also help reconciling mixed findings in literature.

\section{Building Resilience}


From the analysis of the literature, it is now well established that exposure to a disaster increases the risk of developing psychological disorders; however, research on traumatized children has revealed wide variations in outcomes. Despite the numerous consequences, children frequently demonstrate their ability to recover from extreme adversity (Jones, 2008). It is therefore crucial to highlight the factors that can protect children, reduce the impact of stressors, promote capacity for mastery, and help to resume normal functioning (Lazarus \& Folkman, 1984). In this review we have highlighted the need to include the group and intergroup levels of analysis as well as the interaction between all four levels in future research. We argue that this approach allows to capture the multidimensionality and complexity of this context to fully understand how to promote resilience in children and the communities they live in. This is because adaptation to traumatic experiences is a dynamic process involving multiple interacting systems within the individual organism and many interactions, including relationships with other people and the environment (Masten \& Narayan, 2012).

Resilience is defined by the American Psychological Association (2014) as "the process of adapting well in the face of adversity, trauma, tragedy, threats or even significant sources of stress (para. 4)." It refers to the process of adapting well to adversity in life, such as stressful personal life events or disasters, and it can be collective as well as personal (Williams \& Drury, 2010). Resilience factors include a host of biological, psychological, social and cultural factors that interact to determine how a dynamic system responds to stressful experiences adapting successfully to disturbances that threaten the viability, the function, or the development of that system (Masten, 2014).

However, from our analysis, it is clear that the interest of researchers has been on a rather narrow definition of resilience, echoing the literature on trauma more generally. This is because 
outcome variables mostly relate to individual trauma (e.g., depression, PTSD), and predictors or interventions are largely based on individual factors (Tables 1, 6, 7 in OSM). Similarly, interventions to alleviate stress after disaster exposure are targeted at individual level, e.g., Eye Movement Desensitization and Reprocessing (EMDR) or Group Cognitive-Behavioral Interventions (GCBI) (Brown et al., 2017) (Table 3 in OSM). Although disasters lead to individual distress and trauma, distress and trauma are collective and shared by communities as a whole, and we argue for the need to employ actions that can benefit a large number of people. Such actions should not only be "curative" in the sense of targeting individual distress and disorder but, building on a broader understanding of resilience, should support recovery in the community as a whole, for instance promoting reciprocal help, coping, and collective action aimed at obtaining support from relevant institutions and uninvolved bystanders. We therefore argue for the need to rely on the concept proposed by Drury et al. (2019) of collective psychosocial resilience, referring to survivors that face the disaster as a group.

In line with the argument above, the psychological literature on community resilience refers to a network of adaptive capacities, namely community competence, social capital, economic development and communication, that allow the community in which the child lives in to fully recover (Norris et al., 2008). Perceived and actual social support, a sense of a community, collective empowerment and action play important roles in interaction with economic resources or media information. In particular, social capital has been shown to be an efficient source of instrumental and emotional support to establish cohesion. Social capital acts as a bonding source to strengthen existing ties in the family community (Norris et al., 2002; Norris et al., 2008), but also bridges ties to organizations for indirect help, e.g., through church (Hawkins \& Maurer, 2009), and links the community with policy makers (Szreter \& Woolcock, 
2004). Social capital is typically a function of psychological group memberships (Helliwell \& Barrington-Leigh, 2012). Note however that literature on community resilience has been generally detached from the disaster literature, and operationalizations of resilience in terms of community were largely absent from the studies that we reviewed.

Our argument is also in line with research in resilience in development. Factors that enhance child resiliency are effective parenting, positive self-concept, self-regulation, social competence, cognitive flexibility, problem-solving skills, communication skills, empathy, assertiveness, humor, group affiliation, and the ability to elicit caretaking behaviors (Masten, 2007). Furthermore, communities are constituted by several groups such as multiethnic communities but also communities with wealth disparities or vulnerable groups, which should be taken into account when designing interventions.

In our article we argue for the importance of combining individual support with the creation and reinforcement of positive relations between individuals (such as families and peers), within groups, and between groups (such as majority and minority groups). Taking into account all the components that reciprocally interact in a community (and beyond) provides a more powerful way to promote resilience at individual and collective levels (therefore also capitalizing on a more general concept of community resilience, or collective psychosocial resilience; Drury et al., 2019). In doing so, since group membership has an important effect on behavior, and especially collective behavior, future research should build on findings relating to people's social identities and collective empowerment (Drury, 2018).

\section{Conclusion}

We argue that disasters have consequences which are a complex interplay between individual, interpersonal, group, and intergroup factors. Each of the levels contains risk or 
protective factors that can enhance or buffer the direct and indirect consequences of the disaster and lead to resilience. We provided evidence that these levels interact, and that in order to understand children's responses to disasters considering all of them is of vital importance. Our aim is to provide a new perspective that highlights the relevance of all four levels, by offering a wider conceptualization of distress and resilience as determined by multiple individual and social factors. This review hopes to stimulate future research that examines the complex nature of disasters and that eventually allows the development of an integrated model considering all levels. Understanding the consequences of disasters for children is crucial for their development and well-being, as well as for the design of effective interventions that strengthen protective factors in children, families, and the society. 


\section{Footnote}

1. Note that although an individualistic approach considers social support as an interpersonal variable, this may also be a function of group membership (cf. Vezzali et al., 2017).

\section{Funding}

The authors have no funding to disclose.

\section{Conflict of interest}

The authors declare that they have no conflict of interest. 


\section{References}

American Psychological Association. (2014). The road to resilience. Washington, DC: American Psychological Association. Retrieved from http://www.apa.org/helpcenter/roadresilience.aspx

Amin, R., Nadeem, E., Iqbal, K., Asadullah, M. A., \& Hussain, B. (2020). Support for Students Exposed to Trauma (SSET) Program: An Approach for Building Resilience and Social Support Among Flood-Impacted Children. School Mental Health, 12, 493-506. doi:10.1007/s12310-020-09373-y

Andrighetto, L., Vezzali, L., Bergamini, G., Nadi, C., \& Giovannini, D. (2016). Inside the earthquake: Perceived disaster exposure and helping intentions among Italian and immigrant victims of the 2012 Italian earthquakes. Group Processes and Intergroup Relations, 19, 753768. doi:10.1177/1368430215591040

Baggerly, J. N., \& Exum, H. (2008). Counseling children after natural disasters: Guidance for family therapists. American Journal of Family Therapy, 36, 79-93.

Bokszczanin, A. (2007). PTSD symptoms in children and adolescents 28 months after a flood: Age and gender differences. Journal of Traumatic Stress, 20, 347-351. years after the Marmara earthquake. Child and Adolescent Mental Health, 13, 134-139

Bronfenbrenner, U. (1979). The ecology of human development: Experiments by nature and design. Cambridge, MA: Harvard University Press. ISBN 0-674-22457-4.

Bronfenbrenner, U. (2005). Making human beings human: Bioecological perspectives on human development. Thousand Oaks, CA: Sage Publications.

Brown, C. S., Mistry, R. S., \& Bigler, R. S. (2007). Hurricane Katrina: African American Children's Perceptions of Race, Class, and Government Involvement Amid a National Crisis. 
Analyses of Social Issues and Public Policy, 7, 191-208. doi:10.1111/j.15302415.2007.00139.x

Brown, R. C., Witt, A., Fegert, J. M., Keller, F., Rassenhofer, M., \& Plener, P. L. (2017).

Psychosocial interventions for children and adolescents after man-made and natural disasters: a meta-analysis and systematic review. Psychological Medicine, 47, 1893-1905. doi:10.1017/s0033291717000496

Buthmann, J., Finik, J., Ventura, G., Zhang, W., Shereen, A. D., \& Nomura, Y. (2019). The children of Superstorm Sandy: Maternal prenatal depression blunts offspring electrodermal activity. Biological Psychology, 146, Article 107716.

https://doi.org/10.1016/j.biopsycho.2019.107716

Cadamuro, A., Versari, A., Vezzali, L., \& Trifiletti, E. (2016). Preventing the detrimental effect of posttraumatic stress in young children: The role of theory of mind in the aftermath of a natural disaster. European Journal of Developmental Psychology, 13, 52-66. doi:10.1080/17405629.2015.1055240

Cadamuro, A., Versari, A., Vezzali, L., Giovannini, D., \& Trifiletti, E. (2015). Cognitive performance in the aftermath of a natural disaster: The role of coping strategies, theory of mind and peer social support. Child and Youth Care Forum, 44, 93-113. doi:10.1007/s10566$014-9272-z$

Catani, C., Gewirtz, A. H., Wieling, E., Schauer, E., Elbert, T. \& Neuner, F. (2010). Tsunami, war, and cumulative risk in the lives of Sri Lankan schoolchildren. Child Development, 81, 1176-1191. 
Catani, C., Jacob, N., Schauer, E., Kohila, M., \& Neuner, F. (2008). Family violence, war, and natural disasters: A study of the effect of extreme stress on children's mental health in Sri Lanka. BMC Psychiatry, 8, 33. doi:10.1186/1471-244X-8-33

Cheng, J., Liang, Y.-M., Zhou, Y.-Y., Eli, B., \& Liu, Z.-K. (2019). Trajectories of PTSD symptoms among children who survived the Lushan earthquake: a four-year longitudinal study. Journal of Affective Disorders, 252, 421-427. doi:10.1016/j.jad.2019.04.047

Copeland, W. E., Keeler, G., Angold, A., \& Costello, E. J. (2007). Traumatic Events and Posttraumatic Stress in Childhood. Archives of General Psychiatry, 64, 577-584. doi:10.1001/archpsyc.64.5.577

Costa, N., Weems, C., \& Pina, A. (2009). Hurricane Katrina and youth anxiety: the role of perceived attachment beliefs and parenting behaviors. Journal of Anxiety Disorders, 23, 935941.

Cryder, C. H., Kilmer, R. P., Tedeschi, R. G., \& Calhoun, L. G. (2006). An exploratory study of posttraumatic growth in children following a natural disaster. American Journal of Orthopsychiatry, 76, 65- 69.

Danielson, C. K., Cohen, J. R., Adams, Z. W., Youngstrom, E. A., Soltis, K., Amstadter, A. B., \& Ruggiero, K. J. (2016). Clinical Decision-Making Following Disasters: Efficient Identification of PTSD Risk in Adolescents. Journal of Abnormal Child Psychology, 45, $117-$ 129.

De Young, A. C., Kenardy, J. A., \& Cobham, V. E. (2011). Trauma in Early Childhood: A Neglected Population. Clinical Child and Family Psychology Review, 14, 231-250. doi:10.1007/s10567-011-0094-3 
Drury, J. (2018). The role of social identity processes in mass emergency behaviour: An integrative review. European Review of Social Psychology, 29, 38-81. doi:10.1080/10463283.2018.1471948

Drury, J., Carter, H., Cocking, C., Ntontis, E., Guven, S. T., \& Amlot, R. (2019). Facilitating collective psychosocial resilience in the public in emergencies: Twelve recommendations based on the social identity approach. Frontiers in Public Health, 7, 1-21. doi:10.3389/fpubh.2019.00141

Drury, S.S., Scheeringa M.S., \& Zeanah C.H. (2008). The traumatic impact of Hurricane Katrina on children in New Orleans. Child and Adolescent Psychiatric Clinics of North America, 17, $685-702$.

Eksi, A., Braun, K. L., Ertem-Vehid, H., Peyerli, G., Saydam, R., Toparlak, D., \& Alyanak, B. (2007). Risk factors for the development of PTSD and depression among child and adolescent victims following a 7.4 magnitude earthquake. International Journal of Psychiatry in Clinical Practice, 11, 190-199.

Evans, L., \& Oehler-Stinnett, J. (2006). Children and Natural Disasters. School Psychology International, 27, 33-55. doi:10.1177/0143034306062814

Farooqui M., Quadri S.A., Suriya S.S. et al. (2017). Posttraumatic stress disorder: a serious postearthquake complication. Trends in Psychiatry and Psychotherapy, 39, 135-43.

Felix E., Hernández L. A., Bravo M., Ramirez R., Cabiya J., \& Canino G. (2011). Natural disaster and risk of psychiatric disorders in Puerto Rican children. Journal of Abnormal Child Psychology, 39, 589-600. 10.1007/s10802-010-9483-1 
Felix, E., Kaniasty, K., You, S., \& Canino, G. (2015). Parent-child relationship quality and gender as moderators of the influence of hurricane exposure on physical health among children and youth. Journal of Pediatric Psychology, 41, 73-85. doi:10.1093/jpepsy/jsv038

Felix, E., You, S., \& Canino, G. (2013). School and community influences on the long term postdisaster recovery of children and youth following hurricane Georges. Journal of Community Psychology, 41, 1021-1038. doi:10.1002/jcop.21590

Feo, P., Di Gioia, S., Carloni, E., Vitiello, B., Tozzi, A. E., \& Vicari, S. (2014). Prevalence of psychiatric symptoms in children and adolescents one year after the 2009 L'Aquila earthquake. BMC Psychiatry, 14. doi:10.1186/s12888-014-0270-3

Furr, J. M., Comer, J. S., Edmunds, J. M., \& Kendall, P. C. (2010). Disasters and youth: A metaanalytic examination of posttraumatic stress. Journal of Consulting and Clinical Psychology, 78, 765-780. doi:10.1037/a0021482

Gordon-Hollingsworth, A. T., Yao, N., Chen, H., Qian, M., \& Chen, S. (2015). Understanding the Impact of Natural Disasters on Psychological Outcomes in Youth from Mainland China: a Meta-Analysis of Risk and Protective Factors for Post-Traumatic Stress Disorder Symptoms. Journal of Child \& Adolescent Trauma, 11, 205-226.

Han, L., Zhang, Y., \& Zheng, Y. (2012). Responses over time of child and adolescent survivors to the 2008 Wenchuan, China Earthquake. Social Behavior and Personality: An International Journal, 40, 1147-1152. doi:10.2224/sbp.2012.40.7.1147

Hansel, T. C., Osofsky, H. J., Osofsky, J. D., \& Speier, A. H. (2019). Katrina inspired disaster screenings and services: School-based trauma interventions. Traumatology, 25, 133-141. https://doi.org/10.1037/trm0000178 
Hausman, E. M., Black, S. R., Bromet, E., Carlson, G., Danzig, A., Kotov, R., \& Klein, D. N. (2020). Reciprocal effects of maternal and child internalizing symptoms before and after a natural disaster. Journal of family psychology. Advance online publication.

https://doi.org/10.1037/fam0000653

Hawkins, R. L., \& Maurer, K. (2009). Bonding, bridging and linking: How social capital operated in New Orleans following Hurricane Katrina. British Journal of Social Work, 40, 1777-1793. doi:10.1093/bjsw/bcp087

Helliwell J. F., \& Barrington-Leigh, C. P. (2012). How much is social capital worth? In J. Jetten, C. Haslam, \& S. A. Haslam (Eds.), The social cure: Identity, health, and well-being (pp. 5571). Hove, UK: Psychology Press.

Hensley-Maloney, L. \& Varela, R.E. (2009). Child Youth Care Forum, 38, 135-149. https://doi.org/10.1007/s10566-009-9072-z

Hlodversdottir, H., Thorsteinsdottir, H., Thordardottir, E. B., Njardvik, U., Petursdottir, G., \& Hauksdottir, A. (2018). Long-term health of children following the Eyjafjallajökull volcanic eruption: a prospective cohort study. European Journal of Psychotraumatology, 9 (sup2), 1442601.

Jetten, J., Haslam, C, \& Haslam, S. A. (Eds.). (2015). The social cure: Identity, health and wellbeing. Hove, England: Psychology Press.

Jetten, J., Haslam, C., Haslam, S. A., \& Branscombe, N. R. (2009). The social cure. Scientific American Mind, 20, 26-33. doi:10.1038/scientificamericanmind0909-26

Jetten, J., \& Spears, R. (2003). The divisive potential of differences and similarities: The role of intergroup distinctiveness in intergroup differentiation. European Review of Social Psychology, 14, 203-241. doi:10.1080/10463280340000063 
Jones, L. (2008). Responding to the needs of children in crisis. International Review of Psychiatry, 20, 291-303. doi:10.1080/09540260801996081

Jones, R. T., Frary, R., Cunningham, P., Weddle, J. D., \& Kaiser, L. (2001). The psychological effects of Hurricane Andrew on ethnic minority and Caucasian children and adolescents: A case study. Cultural Diversity and Ethnic Minority Psychology, 7, 103-108. doi:10.1037/1099-9809.7.1.103

Kaniasty, K., \& Norris, F. (1999). The experience of disaster: Individuals and communities sharing trauma. In R. Gist \& B. Lubin (Eds.), Response to disaster: Psychosocial, community and ecological approaches (pp. 25-61). Philadelphia, PA: Brunner/Mazel.

Kar, N., Mohapatra, P. K., Nayak, K. C., Pattanaik, P., Swain, S. P., \& Kar, H. C. (2007). Posttraumatic stress disorder in children and adolescents one year after a super-cyclone in Orissa, India: exploring cross-cultural validity and vulnerability factors. BMC Psychiatry, 7. doi:10.1186/1471-244x-7-8

Kessel, E. M., Nelson, B. D., Finsaas, M., Kujawa, A., Meyer, A., Bromet, E., ... Klein, D. N. (2019). Parenting style moderates the effects of exposure to natural disaster-related stress on the neural development of reactivity to threat and reward in children. Development and Psychopathology, 1-10. doi:10.1017/s0954579418001347

Kronenberg, M. E., Hansel, T. C., Brennan, A. M., Osofsky, H. J., Osofsky, J. D., \& Lawrason, B. (2010). Children of Katrina: Lessons learned about postdisaster symptoms and recovery patterns. Child Development, 81, 1241-1259. doi:10.1111/j.1467-8624.2010.01465.x

Kroska, E. B., Miller, M. L., Roche, A. I., Kroska, S. K., \& O'Hara, M. W. (2018). Effects of traumatic experiences on obsessive-compulsive and internalizing symptoms: The role of avoidance and mindfulness. Journal of Affective Disorders, 225, 326-336. 
La Greca, A. M., Silverman, W. K., Lai, B., \& Jaccard, J. (2010). Hurricane-related exposure experiences and stressors, other life events, and social support: Concurrent and prospective impact on children's persistent posttraumatic stress symptoms. Journal of Consulting and Clinical Psychology, 78, 794-805. doi:10.1037/a0020775

La Greca, A.M., Silverman, W.K., \& Wasserstein, S.B. (1998). Children's predisaster functioning hurricane Andrew. Journal of Consulting and Clinical Psychology, 66, 883- 892.

Lai B.S., La Greca A.M., Auslander B.A., \& Short M.B. (2013). Children's symptoms of posttraumatic stress and depression after a natural disaster: Comorbidity and risk factors. Journal of Affective Disorders, 146, 71-78. doi:pii: S0165-0327(12)00612-X

Lazarus. R.S., \& Folkman, S. (1984). Stress, Appraisal and Coping. Springer Publishing.

Le Brocque, R., De Young, A., Montague, G., Pocock, S., March, S., Triggell, N., . . Kenardy, J. (2017). Schools and natural disaster recovery: The unique and vital role that teachers and education professionals play in ensuring the mental health of students following natural disasters. Journal of Psychologists and Counsellors in Schools, 27, 1-23. 10.1017/jgc.2016.17

Li, G., Wang, L., Cao, C., Fang, R., Cao, X., Chen, C., Elhai, J. D., \& Hall, B. J. (2019). Posttraumatic stress disorder and executive dysfunction among children and adolescents: A Latent Profile Analysis. International Journal of Clinical and Health Psychology, 19, 228236. https://doi.org/10.1016/j.ijchp.2019.07.001

March, J. S., Amaya-Jackson, L., Terry, R., \& Costanzo, P. (1997). Posttraumatic Symptomatology in Children and Adolescents After an Industrial Fire. Journal of the American Academy of Child \& Adolescent Psychiatry, 36, 1080-1088. doi:10.1097/00004583199708000-00015

Marsee, M. A. (2008). Reactive aggression and posttraumatic stress in adolescents affected by 
RESILIENCE IN CHILDREN AFTER NATURAL DISASTERS

Hurricane Katrina. Journal of Clinical Child \& Adolescent Psychology, 37, 519-529.

Masten A. S. (2014). Global perspectives on resilience in children and youth. Child Development, 85, 6-20. doi:10.1111/cdev.12205

Masten, A. S. (2007). Resilience in developing systems: Progress and promise as the fourth wave rises. Development and Psychopathology, 19, 921-930. doi:10.1017/s0954579407000442

Masten, A. S., \& Narayan, A. J. (2012). Child development in the context of disaster, war, and terrorism: Pathways of risk and resilience. Annual Review of Psychology, 63, 227-257. doi:10.1146/annurev-psych-120710-100356

Masten, A. S., \& Obradovìc, J. (2008). Disaster preparation and recovery: Lessons from research on resilience in human development. Ecology and Society, 13, 9. doi:10.5751/ES-02282130109

Masten, A. S., \& Osofsky, J. D. (2010). Disasters and their impact on child development: Introduction to the special section. Child Development, 8, 1029-1039. doi:10.1111/j.14678624.2010.01452.x

Mikyung Jang, Se-hwa Lee \& Lee-jin Kim (2020) Post-traumatic stress disorder and behavioral problems of parents and children after the 2015 Nepal earthquakes. International Journal of Mental Health, 49, 3-16, DOI: 10.1080/00207411.2020.1725719

Moher, D., Liberati, A., Tetzlaff, J., Altman D.G, \& The PRISMA Group (2009). Preferred reporting items for systematic reviews and meta-analyses: The PRISMA statement. PLoS Med, 6 (7): e1000097. doi:10.1371/journal.pmed1000097

Mooney, M., Tarrant, R., Paton, D., Johnston, D., \& Johal, S. (2020). The school community contributes to how children cope effectively with a disaster. Pastoral Care in Education, 124. doi:10.1080/02643944.2020.1774632 
Muldoon O. T., Haslam S. A., Haslam C., Cruwys T., Kearns M., \& Jetten J. (2019). The social psychology of responses to trauma: Social identity pathways associated with divergent traumatic responses. European Review of Social Psychology, 30, 311-348. doi:10.1080/10463283.2020.1711628

Nomura, Y., Davey, K., Pehme, P. M., Finik, J., Glover, V., Zhang, W., ... Ham, J. (2019). Influence of in utero exposure to maternal depression and natural disaster-related stress on infant temperament at 6 months: The children of Superstorm Sandy. Infant Mental Health Journal, 40, 204-216.doi:10.1002/imhj.21766

Norris, F. H., Friedman, M. J., Watson, P. J., Byrne, C. M., Diaz, E., \& Kaniasty, K. (2002). 60,000 disaster victims speak: Part I. An empirical review of the empirical literature, 19812001. Psychiatry: Interpersonal and Biological Processes, 65, 207-239. doi:10.1521/psyc.65.3.207.20173

Osofsky, H. J., Osofsky, J. D., Kronenberg, M., Brennan, A., \& Hansel, T. C. (2009). Posttraumatic stress symptoms in Children after Hurricane Katrina: Predicting the need for mental health services. American Journal of Orthopsychiatry, 79, 212-220.

Peek, L. (2008). Children and disasters: Understanding vulnerability, developing capacities, and promoting resilience - an introduction. Children, Youth and Environments, 18, 1-29. doi:10.7721/chilyoutenvi.18.1.0001

Peek, L., Abramson, D. M., Cox, R. S., Fothergill, A., \& Tobin, J. (2018). Children and disasters. In Handbook of disaster research (pp. 243-262). Springer, Cham.

Peek, L., \& Stough, L. M. (2010). Children With Disabilities in the Context of Disaster: A Social Vulnerability Perspective. Child Development, 81, 1260-270. doi:10.1111/j.14678624.2010.01466.x 
Pfefferbaum, B., Seale, T. W., McDonald, N. B., Brandt, E. N., Jr., Rainwater, S. M., Maynard, B. T., .. . Miller, P. D. (2000). Posttraumatic stress two years after the Oklahoma City bombing in youths geographically distant from the explosion. Psychiatry. Interpersonal and Biological Processes, 63, 358-370.

Powell, T., \& Holleran-Steiker, L. K. (2017). Supporting Children After a Disaster: A Case Study of a Psychosocial School-Based Intervention. Clinical Social Work Journal, 45, 176188. doi:10.1007/s10615-015-0557-y

Pullins, L. G., McCammon, S. L., Lamson, A. S., Wuensch, K. L., \& Mega, L. (2005). SchoolBased Post-Flood Screening and Evaluation: Findings and Challenges in One Community. Stress, Trauma, and Crisis, 8, 229-249. doi:10.1080/15434610500406343

Russoniello, C. V., Skalko, T. K., O’Brien, K., McGhee, S. A., Bingham-Alexander, D., \& Beatley, J. (2002). Childhood Posttraumatic Stress Disorder and Efforts to Cope After Hurricane Floyd. Behavioral Medicine, 28, 61-71. doi:10.1080/08964280209596399 Samuelson K.W., Krueger C.E., Burnett C., \& Wilson C.K. (2009). Neuropsychological functioning in children with posttraumatic stress disorder. Child Neuropsychology, 16, 119133. doi: $10.1080 / 09297040903190782$

Saylor, C. F., Cowart, B. L., Lipovsky, J. A., Jackson, C., \& Finch, A. J. (2003). Media exposure to September 11: Elementary school students'experiences and posttraumatic symptoms. American Behavioral Scientist,46, 1622-1642. https://doi.org/10.1177/0002764203254619

Schoeman R, Carey P, Seedat S. (2009). Trauma and posttraumatic stress disorder in South African adolescents: A case-control study of cognitive deficits. Journal of Nervous and Mental Disease, 197, 244-250. doi: 10.1097/NMD.0b013e31819d9533 
Shaffer, D.R., \& Kipp, K. (2009). Developmental psychology: Childhood and adolescence (8th ed.). Belmont, CA: Wadsworth.

Shannon, M. P., Lonigan, C. J., Finch, A. J., \& Taylor, C. M. (1994). Children Exposed to Disaster: I. Epidemiology of Post-Traumatic Symptoms and Symptom Profiles. Journal of the American Academy of Child \& Adolescent Psychiatry, 33, 80-93. doi:10.1097/00004583199401000-00012

Shelton, J. N., Richeson, J. A., \& Vorauer, J. D. (2006). Threatened identities and interethnic interactions. European Review of Social Psychology, 17, 321-358.

doi:10.1080/10463280601095240

Silverman, W. K., La Greca, A. M. (2002). Children experiencing disasters: Definitions, reactions, and predictors of outcomes. In La Greca, Annette M. (Ed); Silverman, Wendy K. (Ed); Vernberg, Eric M. (Ed) \& Roberts, Michael C. (Ed). (2002). Helping children cope with disasters and terrorism, (pp. 11-33). Washington, DC, US: American Psychological Association, xvii, 446 pp.

Silwal S, Dybdahl R, Chudal R, Sourander A, Lien L. (2018). Psychiatric symptoms experienced by adolescents in Nepal following the 2015 earthquakes. Journal of Affective Disorders, 234,239-246. https://doi.org/10.1016/j.jad.2018.03.002

Simcock, G., Cobham, V. E., Laplante, D. P., Elgbeili, G., Gruber, R., Kildea, S., \& King, S. (2019). A cross-lagged panel analysis of children's sleep, attention, and mood in a prenatally stressed cohort: The QF2011 Queensland flood study. Journal of Affective Disorders, 255, 96104. doi:10.1016/j.jad.2019.05.041 
Sprung, M. (2008). Unwanted Intrusive Thoughts and Cognitive Functioning in Kindergarten and Young Elementary School-Age Children Following Hurricane Katrina. Journal of Clinical Child \& Adolescent Psychology, 37, 575-587. doi:10.1080/15374410802148236

Staub, E. (2003). The psychology of good and evil: Why children, adults, and groups help and harm others. Cambridge, UK: Cambridge University Press.

Strahm, A. M., Bagne, A. G., Rued, H. A., Larson, K. J., Roemmich, J. N., \& Hilmert, C. J. (2020). Prenatal traumatic stress and offspring hair cortisol concentration: A nine year follow up to the Red River flood pregnancy study. Psychoneuroendocrinology, 113, 104579. doi:10.1016/j.psyneuen.2019.104579

Szreter, S., \& Woolcock, M. (2004). Rejoinder: Crafting rigorous and relevant social theory for public health policy. International Journal of Epidemiology, 33, 700-704. doi:10.1093/ije/dyh263

Tajfel, H., \& Turner, J. C. (1979). An integrative theory of intergroup conflict. In W. G. Austin, \& S. Worchel (Eds.), The social psychology of intergroup relations (pp. 33-47). Monterey, CA US: Brooks/Cole.

Takada, S. (2013). Post-Traumatic Stress Disorders and mental health care (lessons learned from the Hanshin-Awaji Earthquake, Kobe, 1995). Brain and Development, 35, 214-219. doi:10.1016/j.braindev.2012.09.013

Tang, B., Liu, X., Liu, Y., Xue, C., Zhang, L. (2014). A meta-analysis of risk factors for depression in adults and children after natural disasters. BMC Public Health, 14, 623.doi:10.1186/1471-2458-14-623 
Tang, W., Xu, D., Li, B., Lu, Y., \& Xu, J. (2018). The relationship between the frequency of suicidal ideation and sleep disturbance factors among adolescent earthquake victims in China. General Hospital Psychiatry, 55, 90-97. doi:10.1016/j.genhosppsych.2018.09.013

Tanner, T., \& Seballos, F. (2012). Action research with children: Lessons from tackling disasters and climate change. IDS Bulletin, 43, 59-57.

Tao, T., X. Duan, and J. Shi (2014) 'Posttraumatic stress symptoms of Chinese rural children and adolescents surviving the 2008 Wenchuan earthquake assessed using CRIES'. Journal of Loss and Trauma, 19, 1-11.

Taylor, S. E., \& Stanton, A. L. (2007). Coping Resources, Coping Processes, and Mental Health. Annual Review of Clinical Psychology, 3, 377-401.

doi:10.1146/annurev.clinpsy.3.022806.091520

Terranova, A. M., Boxer, P., \& Morris, A. S. (2009). Factors influencing the course of posttraumatic stress following a natural disaster: Children's reactions to Hurricane Katrina. Journal of Applied Developmental Psychology, 30, 344-355. doi:10.1016/j.appdev.2008.12.017

Terranova, A. S. Morris, S. Myers, M. Kithakya, \& Michael Morris. (2015). Preschool Children's Adjustment Following a Hurricane: Risk and Resilience in the Face of Adversity. Early Education and Development, 26, 534-548.

Turner, J. C., Hogg, M. A., Oakes, P. J., Reicher, S. D., \& Wetherell, M. S. (1987). Rediscovering the social group: A self-categorization theory. Oxford, UK: Blackwell.

Usami, M., Iwadare, Y., Ushijima, H., Inazaki, K., Tanaka, T., Kodaira, M., ... Saito, K. (2019). Did kindergarteners who experienced the Great East Japan earthquake as infants develop 
traumatic symptoms? Series of questionnaire-based cross-sectional surveys. Asian Journal of Psychiatry, 44, 38-44. doi:10.1016/j.ajp.2019.07.011

Vezzali, L., Andrighetto, L., Drury, J., Di Bernardo, G. A., \& Cadamuro, A. (2017). In the aftermath of natural disasters: Fostering helping towards outgroup victims. In: van Leeuwen E., Zagefka H. (Eds), Intergroup Helping (pp. 305-330). Springer, Cham. doi:10.1007/978-3319-53026-0_15

Vezzali, L., Cadamuro, A., Versari, A., Giovannini, D., \& Trifiletti, E. (2015). Feeling like a group after a natural disaster: Common ingroup identity and relations with outgroup victims among majority and minority young children. British Journal of Social Psychology, 54, 519538. doi:10.1111/bjso.12091

Vezzali, L., Drury, J., Cadamuro, A., \& Versari, A. (2016). Sharing distress increases helping and contact intentions via one-group representation and inclusion of the other in the self: Children's prosocial behaviour after an earthquake. Group Processes and Intergroup Relations, 19, 314-327. doi:10.1177/1368430215590492

Wasserstein, S. B., \& La Greca, A. M. (1998). Hurricane Andrew: Parent conflict as a moderator of children's adjustment. Hispanic Journal of Behavioral Sciences, 20, 212-224. doi:10.1177/07399863980202005

Weems, C. F., Pina, A. A., Costa, N. M., Watts, S. E., Taylor, L. K., \& Cannon, M. F. (2007). Predisaster trait anxiety and negative affect predict posttraumatic stress in youths after Hurricane Katrina. Journal of Consulting and Clinical Psychology, 75, 154-159. doi:10.1037/0022-006x.75.1.154

Williams, R., \& Drury, J. (2010). The nature of psychosocial resilience and its significance for managing mass emergencies, disasters and terrorism. In: Awotona A. (Ed.), Rebuilding 
sustainable communities for children and their families after disasters: A global survey (pp. 121-148). Newcastle-upon-Tyne, UK: Cambridge Scholars Publishing.

Wolmer L, Laor N, Yazgan Y. (2003). School reactivation programs after disaster: Could teachers serve as clinical mediators? Child and Adolescent Psychiatric Clinics of North America, 12, 363-381. 


\section{RESILIENCE IN CHILDREN AFTER NATURAL DISASTERS}

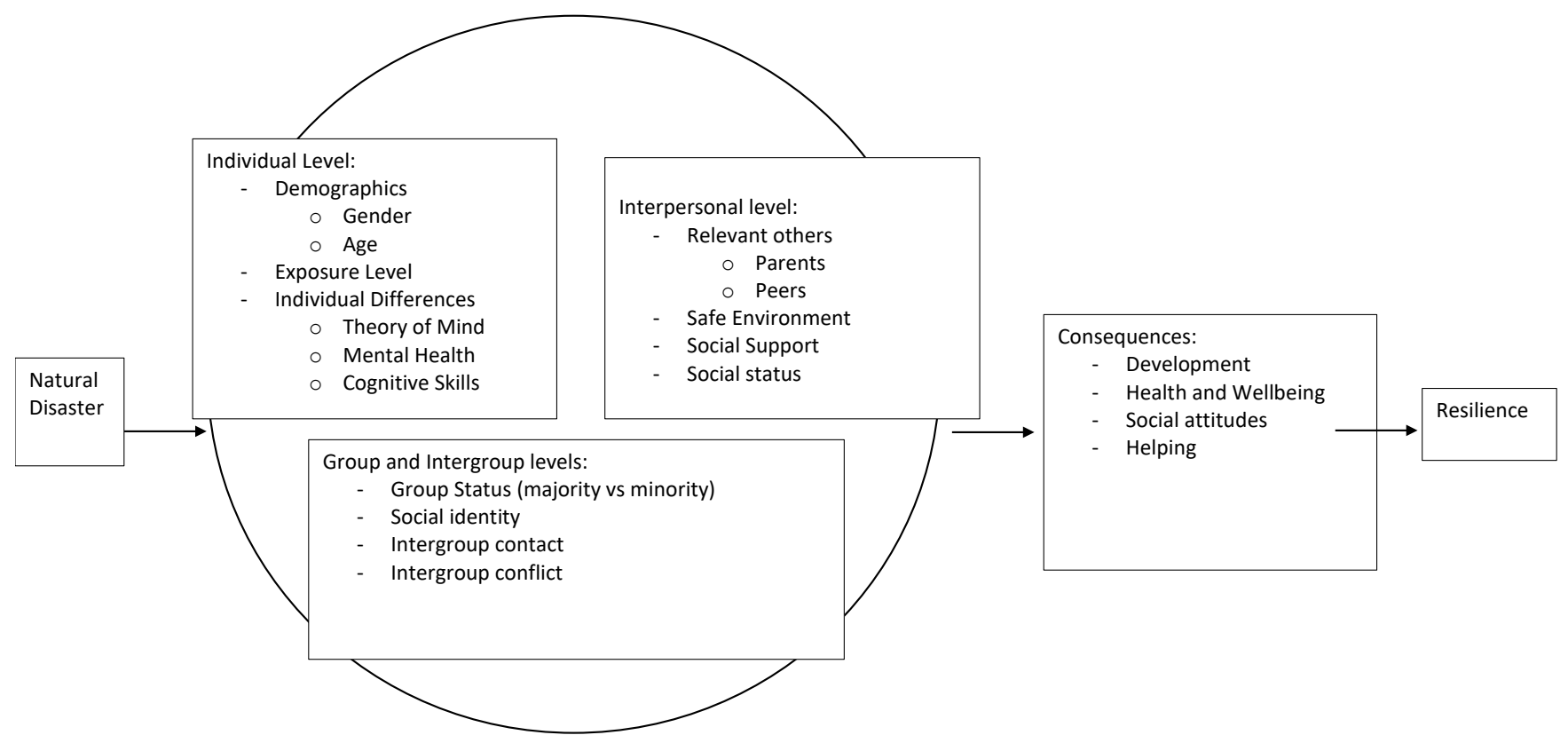

Figure 1. Proposed integrative model. 


\section{Online Supplementary Material}

Figure 1_OSM. Process followed in selecting articles for the review.

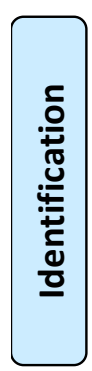

Records identified through database searching $(n=2.055)$

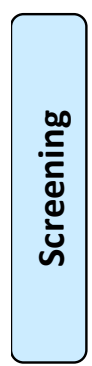

Records after duplicates removed $(n=1.792)$
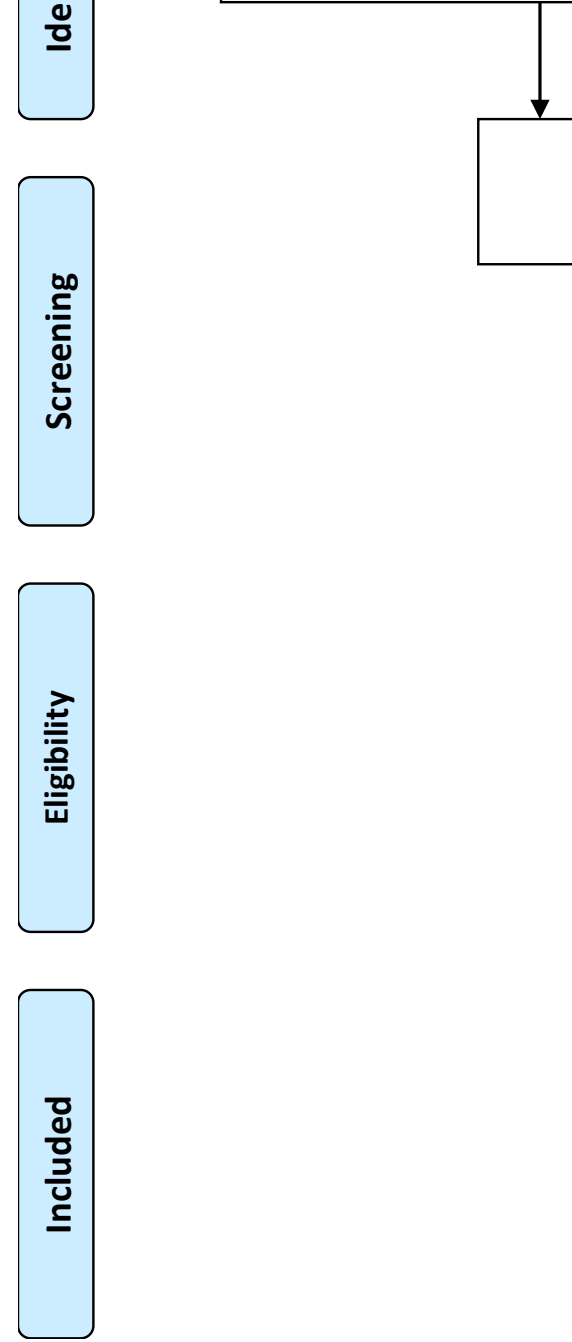

Full-text articles excluded,

Full-text articles assessed for eligibility $(n=572)$ with reasons $(n=278)$

Studies included in review synthesis

$(n=294)$ 
Table 1_OSM. Studies included in the Review ( $N=294$ studies)

\begin{tabular}{|c|c|c|c|c|c|c|c|c|c|}
\hline $\begin{array}{l}\text { Authors and } \\
\text { year }\end{array}$ & $\begin{array}{l}\text { Type of } \\
\text { disaster } \\
\text { (country) }\end{array}$ & Method & Level(s) & Population & $\begin{array}{l}\text { Independen } \\
\text { t } \\
\text { Variable(s) }\end{array}$ & $\begin{array}{l}\text { Mediator( } \\
\text { s) }\end{array}$ & $\begin{array}{l}\text { Moderator( } \\
\text { s) }\end{array}$ & $\begin{array}{l}\text { Assessment(s) } \\
\text { (months } \\
\text { postdisaster) }\end{array}$ & $\begin{array}{l}\text { Dependen } \\
\mathbf{t} \\
\text { Variable(s } \\
\text { ) }\end{array}$ \\
\hline $\begin{array}{l}\text { Adebäck, P., } \\
\text { Schulman, A., } \\
\text { \& Nilsson, D. } \\
\text { (2017). }\end{array}$ & $\begin{array}{l}\text { Tsunami } \\
\text { (Thailand) }\end{array}$ & $\begin{array}{l}\text { Longitudin } \\
\text { al }\end{array}$ & Individual & $\begin{array}{l}210 \text { Swedish } \\
\text { children } \\
\text { aged } 10-15 \\
\text { years }\end{array}$ & $\begin{array}{l}\text { Disaster } \\
\text { exposure, } \\
\text { gender, } \\
\text { age, } \\
\text { education } \\
\text { and living } \\
\text { situation }\end{array}$ & & & $\begin{array}{l}8 \text { years post } \\
\text { disaster }\end{array}$ & $\begin{array}{l}\text { PTSS, } \\
\text { depression, } \\
\text { anxiety } \\
\text { thoughts or } \\
\text { attempted } \\
\text { suicide, } \\
\text { physical } \\
\text { symptoms } \\
\text { or daily } \\
\text { functionin } \\
\mathrm{g}\end{array}$ \\
\hline $\begin{array}{l}\text { Agustini, E. } \\
\text { N., Asniar, I., } \\
\text { \& Matsuo, H. } \\
\text { (2011). }\end{array}$ & $\begin{array}{l}\text { Tsunami } \\
\text { (Indonesia) }\end{array}$ & $\begin{array}{l}\text { Longitudin } \\
\text { al }\end{array}$ & $\begin{array}{l}\text { Individual } \\
\text { and } \\
\text { Interpersona } \\
1\end{array}$ & $\begin{array}{l}482 \\
\text { adolescent } \\
\text { aged 11-19 } \\
\text { years }\end{array}$ & $\begin{array}{l}\text { Gender, loss } \\
\text { of parents, } \\
\text { low support } \\
\text { level and } \\
\text { heavy } \\
\text { somatic } \\
\text { response } \\
\text { age, school } \\
\text { grade, } \\
\text { traumatic } \\
\text { experience }\end{array}$ & & & $\begin{array}{l}4.5 \text { years } \\
\text { post disaster }\end{array}$ & PTSD \\
\hline $\begin{array}{l}\text { Andrades, M., } \\
\text { García, F. E., } \\
\text { Calonge, I., \& } \\
\text { Martínez- } \\
\text { Arias, R. } \\
(2018) .\end{array}$ & $\begin{array}{l}\text { Earthquake } \\
\text { (Chile) }\end{array}$ & $\begin{array}{l}\text { Cross- } \\
\text { sectional }\end{array}$ & Individual & $\begin{array}{l}325 \text { children } \\
\text { aged } 10-15 \\
\text { years }\end{array}$ & $\begin{array}{l}\text { Disaster } \\
\text { exposure, } \\
\text { age, gender, } \\
\text { city of } \\
\text { residence } \\
\text { at the time } \\
\text { of the } \\
\text { earthquake, }\end{array}$ & $\begin{array}{l}\text { Ruminatio } \\
\mathrm{n}\end{array}$ & & $\begin{array}{l}12 \text { months } \\
\text { post disaster }\end{array}$ & $\begin{array}{l}\text { Posttrauma } \\
\text { tic Growth } \\
\text { PTSD }\end{array}$ \\
\hline
\end{tabular}




\begin{tabular}{|c|c|c|c|c|c|c|c|}
\hline & & & & & $\begin{array}{l}\text { and city of } \\
\text { current } \\
\text { residence }\end{array}$ & & \\
\hline $\begin{array}{l}\text { Andrades, M., } \\
\text { García, F. E., } \\
\text { Reyes-Reyes, } \\
\text { A., Martínez- } \\
\text { Arias, \& R., } \\
\text { Calonge, I. } \\
(2016) .\end{array}$ & $\begin{array}{l}\text { Tsunami } \\
\text { (Chile) }\end{array}$ & $\begin{array}{l}\text { Cross- } \\
\text { sectional }\end{array}$ & Individual & $\begin{array}{l}393 \text { children } \\
\text { aged } 10-15 \\
\text { years }\end{array}$ & $\begin{array}{l}\text { Disaster } \\
\text { exposure } \\
\text { age, gender, } \\
\text { city of } \\
\text { residence }\end{array}$ & $\begin{array}{l}20-30 \text { months } \\
\text { post disaster }\end{array}$ & $\begin{array}{l}\text { Posttrauma } \\
\text { tic Growth } \\
\text { PTSD }\end{array}$ \\
\hline $\begin{array}{l}\text { Anthony, J. } \\
\text { L., Lonigan, } \\
\text { C. J., \& } \\
\text { Hecht, S. A. } \\
\text { (1999). }\end{array}$ & $\begin{array}{l}\text { Hurricane } \\
\text { (USA) }\end{array}$ & $\begin{array}{l}\text { Cross- } \\
\text { sectional }\end{array}$ & Individual & $\begin{array}{l}5664 \\
\text { children and } \\
\text { adolescent } \\
\text { aged 9-19 } \\
\text { years }\end{array}$ & $\begin{array}{l}\text { Anxiety, } \\
\text { degree of } \\
\text { disaster } \\
\text { exposure }\end{array}$ & $\begin{array}{l}3 \text { months } \\
\text { post disaster }\end{array}$ & PTSD \\
\hline $\begin{array}{l}\text { Arnberg, F. } \\
\text { K., } \\
\text { Gudmundsdót } \\
\text { tiR., } \\
\text { Butwicka, A., } \\
\text { Fang, F., } \\
\text { Lichtenstein, } \\
\text { P., Hultman, } \\
\text { C. M., \& } \\
\text { Valdimarsdótt } \\
\text { ir, U. A. } \\
\text { (2015). }\end{array}$ & $\begin{array}{l}\text { Tsunami } \\
\text { (Sweden) }\end{array}$ & $\begin{array}{l}\text { Longitudin } \\
\text { al }\end{array}$ & $\begin{array}{l}\text { Individual } \\
\text { and } \\
\text { Interpersona } \\
1\end{array}$ & $\begin{array}{l}8762 \text { adults } \\
3742 \\
\text { children } \\
864088 ; \\
\text { unexposed } \\
\text { adults; } \\
320828 \\
\text { unexposed } \\
\text { children } \\
\text { aged } 12 \\
\text { years }\end{array}$ & $\begin{array}{l}\text { Disaster } \\
\text { exposure, } \\
\text { pre-tsunami } \\
\text { psychiatric } \\
\text { disorders, } \\
\text { age, } \\
\text { gender, } \\
\text { parental pre- } \\
\text { tsunami } \\
\text { disorders, } \\
\text { socioeconom } \\
\text { ic } \\
\text { characteristi } \\
\text { cs }\end{array}$ & $\begin{array}{l}3 \text { and } 11 \\
\text { months } \\
\text { post disaster } \\
\text { (T1-T2) } \\
23 \text { and } 60 \\
\text { months } \\
\text { post disaster } \\
\text { (T3-T4) }\end{array}$ & $\begin{array}{l}\text { Psychiatric } \\
\text { disorders } \\
\text { suicide } \\
\text { attempts }\end{array}$ \\
\hline $\begin{array}{l}\text { Asarnow, J., } \\
\text { Glynn, S., } \\
\text { Pynoos, R. S., } \\
\text { Nahum, J., } \\
\text { Guthrie, D., } \\
\text { Cantwell, D. } \\
\text { P., \& } \\
\text { Franklin, B. } \\
(1999) .\end{array}$ & $\begin{array}{l}\text { Earthquake } \\
\text { (USA) }\end{array}$ & $\begin{array}{l}\text { Cross- } \\
\text { sectional }\end{array}$ & Individual & $\begin{array}{l}63 \text { children } \\
(8.59-18.60 \\
\text { years }) \\
\text { Preselected } \\
\text { for } \\
\text { depression, } \\
\text { attention- } \\
\text { deficit }\end{array}$ & $\begin{array}{l}\text { Disaster } \\
\text { exposure, } \\
\text { predisaster } \\
\text { depression, } \\
\text { predisaster } \\
\text { anxiety } \\
\text { disorder, } \\
\text { pre disaster } \\
\text { psychiatric } \\
\text { disorder, }\end{array}$ & $\begin{array}{l}12 \text { months } \\
\text { post disaster }\end{array}$ & PTSD \\
\hline
\end{tabular}




\begin{tabular}{|c|c|c|c|c|c|c|c|c|}
\hline & & & & $\begin{array}{l}\text { hyperactivity } \\
\text { disorder } \\
\text { and siblings } \\
\text { not } \\
\text { preselected } \\
\text { for the } \\
\text { presence of } \\
\text { psychopatho } \\
\text { lgy }\end{array}$ & $\begin{array}{l}\text { social } \\
\text { impairment, } \\
\text { coping } \\
\text { strategies }\end{array}$ & & & \\
\hline $\begin{array}{l}\text { Ayub, M., } \\
\text { Poongan, I., } \\
\text { Masood, K., } \\
\text { Gul, H., Ali, } \\
\text { M., Farrukh, } \\
\text { A., Shaheen, } \\
\text { A., Chaudhry, } \\
\text { H. \& R., } \\
\text { Naeem, F. } \\
(2012) .\end{array}$ & $\begin{array}{l}\text { Earthquake } \\
\text { (Pakistan) }\end{array}$ & $\begin{array}{l}\text { Cross- } \\
\text { sectional }\end{array}$ & $\begin{array}{l}\text { Individual } \\
\text { and } \\
\text { Interpersona } \\
1\end{array}$ & $\begin{array}{l}1100 \\
\text { children, } \\
\text { aged } 7-16 \\
\text { years }\end{array}$ & $\begin{array}{l}\text { Age, gender, } \\
\text { demographic } \\
\text { socioeconom } \\
\text { ic factors, } \\
\text { family } \\
\text { trauma } \\
\text { related } \\
\text { factors, } \\
\text { psychologica } \\
\text { l } \\
\text { morbidity }\end{array}$ & & $\begin{array}{l}18 \text { months } \\
\text { post disaster }\end{array}$ & $\begin{array}{l}\text { PTSD and } \\
\text { behavioura } \\
1 \text { and } \\
\text { emotional } \\
\text { problems }\end{array}$ \\
\hline $\begin{array}{l}\text { Azarian, A., } \\
\text { Miller, T. W., } \\
\& \\
\text { Skriptchenko- } \\
\text { Gregorian, V. } \\
(1996) .\end{array}$ & $\begin{array}{l}\text { Earthquake } \\
\text { (Armenia) }\end{array}$ & $\begin{array}{l}\text { Cross- } \\
\text { sectional }\end{array}$ & Individual & $\begin{array}{l}90 \text { children } \\
\text { aged } 15-48 \\
\text { month }\end{array}$ & $\begin{array}{l}\text { Disaster } \\
\text { exposure, } \\
\text { gender }\end{array}$ & & $\begin{array}{l}6 \text { months post } \\
\text { disaster }\end{array}$ & 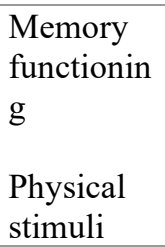 \\
\hline
\end{tabular}




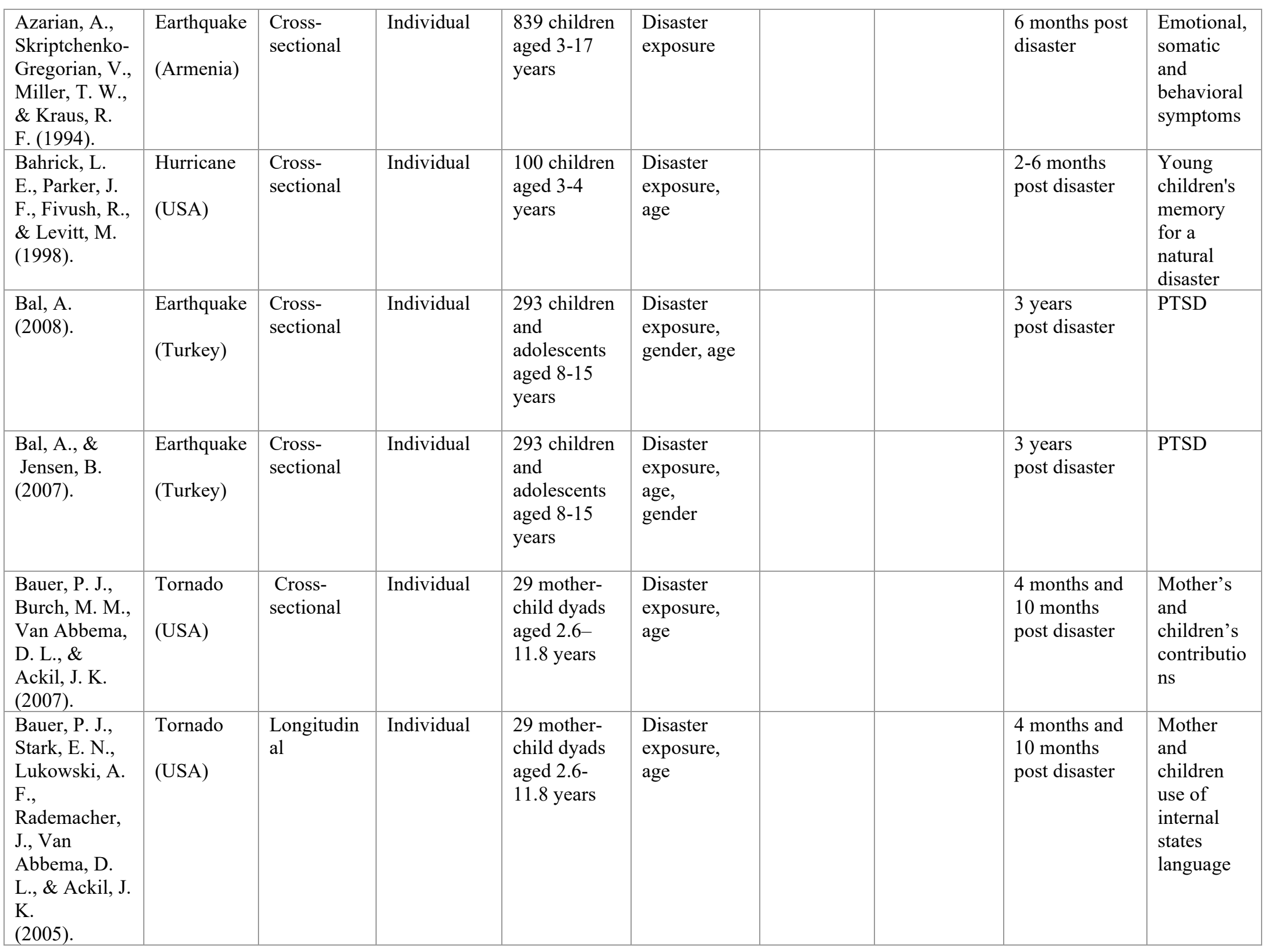




\section{RESILIENCE IN CHILDREN AFTER NATURAL DISASTERS}

\begin{tabular}{|c|c|c|c|c|c|c|c|c|}
\hline $\begin{array}{l}\text { Belter, R. W., } \\
\text { Dunn, S. E., } \\
\& \\
\text { Jeney, P. } \\
\text { (1991). }\end{array}$ & $\begin{array}{l}\text { Hurricane } \\
\text { (USA) }\end{array}$ & $\begin{array}{l}\text { Cross- } \\
\text { sectional }\end{array}$ & Individual & $\begin{array}{l}260 \text { children, } \\
\text { aged } 3-5 \\
\text { years }\end{array}$ & $\begin{array}{l}\text { Disaster } \\
\text { exposure, } \\
\text { demographic } \\
\text { information }\end{array}$ & & $\begin{array}{l}5 \text { months } \\
\text { post disaster }\end{array}$ & $\begin{array}{l}\text { Depression } \\
\text { PTSD } \\
\text { Adjustmen } \\
\mathrm{t}\end{array}$ \\
\hline $\begin{array}{l}\text { Bhushan, B., } \\
\& \\
\text { Sathya- } \\
\text { Kumar, J. } \\
\text { (2007). }\end{array}$ & $\begin{array}{l}\text { Tsunami } \\
\text { (India) }\end{array}$ & $\begin{array}{l}\text { Cross- } \\
\text { sectional }\end{array}$ & Individual & $\begin{array}{l}130 \text { children } \\
\text { aged 10-16 } \\
\text { years }\end{array}$ & $\begin{array}{l}\text { Gender, age } \\
\text { Type of } \\
\text { family, } \\
\text { loss of } \\
\text { family } \\
\text { members, }\end{array}$ & & $\begin{array}{l}12 \text { months } \\
\text { post disaster }\end{array}$ & $\begin{array}{l}\text { Emotional } \\
\text { distress } \\
\text { and } \\
\text { posttrauma } \\
\text { tic } \\
\text { stress in } \\
\text { children }\end{array}$ \\
\hline $\begin{array}{l}\text { Blanc, J., Bui, } \\
\text { E., } \\
\text { Mouchenik, } \\
\text { Y., Derivois, } \\
\text { D., \& Birmes, } \\
\text { P. (2015). }\end{array}$ & $\begin{array}{l}\text { Earthquake } \\
\text { (Haiti) }\end{array}$ & $\begin{array}{l}\text { Cross- } \\
\text { sectional }\end{array}$ & $\begin{array}{l}\text { Individual } \\
\text { and } \\
\text { Interpersona } \\
1\end{array}$ & $\begin{array}{l}96 \text { children } \\
\text { aged } 7-13 \\
\text { years }\end{array}$ & $\begin{array}{l}\text { Social } \\
\text { support } v s \\
\text { no social } \\
\text { support } \\
\text { (control } \\
\text { group) }\end{array}$ & & $\begin{array}{l}12 \text { months } \\
\text { post disaster }\end{array}$ & $\begin{array}{l}\text { PTSD } \\
\text { and } \\
\text { depression }\end{array}$ \\
\hline $\begin{array}{l}\text { Bödvarsdóttir, } \\
\text { Í., Elklit, A, } \\
\& \\
\text { Gudmundsdót } \\
\text { tir, D. B. } \\
(2006) \text {. }\end{array}$ & $\begin{array}{l}\text { Earthquake } \\
\text { (Iceland) }\end{array}$ & $\begin{array}{l}\text { Cross- } \\
\text { sectional }\end{array}$ & Individual & $\begin{array}{l}67 \text { exposed } \\
\text { and } \\
73 \text { no } \\
\text { exposed } \\
\text { children } \\
\text { aged } 10-15 \\
\text { years }\end{array}$ & $\begin{array}{l}\text { Disaster } \\
\text { exposure, } \\
\text { demographic } \\
\text { information, } \\
\text { stressors } \\
\text { relating to } \\
\text { the disaster, } \\
\text { academic } \\
\text { skills and } \\
\text { behavioural } \\
\text { adjustment } \\
\text { in class, } \\
\text { social } \\
\text { support }\end{array}$ & & $\begin{array}{l}3 \text { months } \\
\text { post disaster }\end{array}$ & $\begin{array}{l}\text { PTSD } \\
\text { Psychologi } \\
\text { cal distress }\end{array}$ \\
\hline $\begin{array}{l}\text { Bokszczanin, } \\
\text { A. }(2008) .\end{array}$ & $\begin{array}{l}\text { Flood } \\
\text { (Poland) }\end{array}$ & $\begin{array}{l}\text { Cross- } \\
\text { sectional }\end{array}$ & $\begin{array}{l}\text { Individual } \\
\text { and } \\
\text { Interpersona } \\
1\end{array}$ & $\begin{array}{l}533 \text { children } \\
\text { and } \\
\text { adolescents } \\
\text { age } \mathrm{M}= \\
15.96\end{array}$ & $\begin{array}{l}\text { Gender } \\
\text { age } \\
\text { place of } \\
\text { residence, } \\
\text { exposure to } \\
\text { trauma }\end{array}$ & $\begin{array}{l}\text { Parental } \\
\text { support, } \\
\text { family } \\
\text { conflict, } \\
\text { overprotecti } \\
\text { veness }\end{array}$ & $\begin{array}{l}28 \text { months } \\
\text { post disaster }\end{array}$ & PTSD \\
\hline
\end{tabular}


RESILIENCE IN CHILDREN AFTER NATURAL DISASTERS

\begin{tabular}{|c|c|c|c|c|c|c|c|c|}
\hline $\begin{array}{l}\text { Bradburn, I. } \\
\text { S. (1991). }\end{array}$ & $\begin{array}{l}\text { Earthquake } \\
\text { (USA) }\end{array}$ & $\begin{array}{l}\text { Cross- } \\
\text { sectional }\end{array}$ & Individual & $\begin{array}{l}22 \text { children } \\
\text { aged } 10-12 \\
\text { years }\end{array}$ & $\begin{array}{l}\text { Experience } \\
\text { of and } \\
\text { proximity to } \\
\text { loss, } \\
\text { family } \\
\text { reactions } \\
\text { previously } \\
\text { traumatic } \\
\text { event }\end{array}$ & & $\begin{array}{l}6-8 \text { months } \\
\text { post disaster }\end{array}$ & PTSD \\
\hline $\begin{array}{l}\text { Brown, C. S., } \\
\text { Mistry, R. S., } \\
\text { \& Bigler, R. } \\
\text { S. (2007). }\end{array}$ & $\begin{array}{l}\text { Hurricane } \\
\text { (USA) }\end{array}$ & $\begin{array}{l}\text { Cross- } \\
\text { sectional }\end{array}$ & $\begin{array}{l}\text { Individual } \\
\text { and } \\
\text { Intergroup }\end{array}$ & $\begin{array}{l}77 \text { african } \\
\text { americans } \\
\text { children } \\
\text { aged } 6-14 \\
\text { years }\end{array}$ & Attributions & $\begin{array}{l}\text { Age } \\
\text { victim group } \\
\text { (race class) } \\
\text { socialization }\end{array}$ & $\begin{array}{l}\text { within } 3 \\
\text { months } \\
\text { post disaster }\end{array}$ & $\begin{array}{l}\text { Type of } \\
\text { attribution, } \\
\text { aid and } \\
\text { effectivene } \\
\text { ss } \\
\text { of the } \\
\text { governmen } \\
\text { t }\end{array}$ \\
\hline $\begin{array}{l}\text { Bulut, S. } \\
\text { (2013). }\end{array}$ & $\begin{array}{l}\text { Earthquake } \\
\text { (Turkey) }\end{array}$ & $\begin{array}{l}\text { Cross- } \\
\text { sectional }\end{array}$ & Individual & $\begin{array}{l}191 \text { children } \\
\text { aged } 12 \\
\text { years }\end{array}$ & $\begin{array}{l}\text { Gender } \\
\text { PTSD }\end{array}$ & & $\begin{array}{l}12 \text { months } \\
\text { post disaster }\end{array}$ & $\begin{array}{l}\text { Emotional } \\
\text { and } \\
\text { behavioral } \\
\text { disorders }\end{array}$ \\
\hline $\begin{array}{l}\text { Bulut, S. } \\
\text { (2006). }\end{array}$ & $\begin{array}{l}\text { Earthquake } \\
\text { (Turkey) }\end{array}$ & $\begin{array}{l}\text { Cross- } \\
\text { sectional }\end{array}$ & Individual & $\begin{array}{l}400 \text { children } \\
\text { aged } 9-12 \\
\text { years }\end{array}$ & $\begin{array}{l}\text { Disaster } \\
\text { exposure }\end{array}$ & & $\begin{array}{l}11 \text { months } \\
\text { post disaster }\end{array}$ & PTSD \\
\hline $\begin{array}{l}\text { Bulut, S., } \\
\text { Bulut, S., \& } \\
\text { Tayli, A. } \\
\text { (2005). }\end{array}$ & $\begin{array}{l}\text { Earthquake } \\
\text { (Turkey) }\end{array}$ & $\begin{array}{l}\text { Cross- } \\
\text { sectional }\end{array}$ & Individual & $\begin{array}{l}200 \text { children } \\
4 \text { th and } 5 \text { th } \\
\text { grade }\end{array}$ & $\begin{array}{l}\text { High-impact } \\
\text { trauma and } \\
\text { low-impact } \\
\text { trauma } \\
\text { groups }\end{array}$ & & $\begin{array}{l}11 \text { months } \\
\text { post disaster }\end{array}$ & PTSD \\
\hline $\begin{array}{l}\text { Burke Jr, J. } \\
\text { D., Borus, J. } \\
\text { F., Burns, B. } \\
\text { J., Millstein, } \\
\text { K. H., \& } \\
\text { Beasley, M. } \\
\text { C. (1982). }\end{array}$ & $\begin{array}{l}\text { Storm } \\
\text { (USA) }\end{array}$ & $\begin{array}{l}\text { Longitudin } \\
\text { al }\end{array}$ & Individual & $\begin{array}{l}64 \text { children } \\
\text { age } M=6,5 \\
\text { years }\end{array}$ & $\begin{array}{l}\text { Storm- } \\
\text { related } \\
\text { problems, } \\
\text { age, } \\
\text { time }\end{array}$ & & $\begin{array}{l}6 \text { months } \\
\text { post disaster } \\
5 \text { months } \\
\text { post disaster }\end{array}$ & $\begin{array}{l}\text { Externalizi } \\
\text { ng } \\
\text { behaviour } \\
\text { School } \\
\text { Behavior } \\
\text { Anxiety }\end{array}$ \\
\hline
\end{tabular}




\section{RESILIENCE IN CHILDREN AFTER NATURAL DISASTERS}

\begin{tabular}{|c|c|c|c|c|c|c|c|}
\hline $\begin{array}{l}\text { Burke Jr, J. } \\
\text { D., Moccia, } \\
\text { P., Borus, J. } \\
\text { F., \& Burns, } \\
\text { B. J. (1986). }\end{array}$ & $\begin{array}{l}\text { Flood } \\
\text { (USA) }\end{array}$ & $\begin{array}{l}\text { Cross- } \\
\text { sectional }\end{array}$ & Individual & $\begin{array}{l}47 \text { children } \\
5 \text { th graders }\end{array}$ & $\begin{array}{l}\text { Disaster } \\
\text { exposure, } \\
\text { flood vs no- } \\
\text { flood, } \\
\text { gender }\end{array}$ & $\begin{array}{l}10 \text { months } \\
\text { post disaster }\end{array}$ & Distress \\
\hline $\begin{array}{l}\text { Burnham, J. } \\
\text { J., Hooper, L. } \\
\text { M., Edwards, } \\
\text { E. E., Tippey, } \\
\text { J. M., } \\
\text { McRaney, A. } \\
\text { C., Morrison, } \\
\text { M. A., ... \& } \\
\text { Woodroof, E. } \\
\text { K. } \\
(2008) \text {. }\end{array}$ & $\begin{array}{l}\text { Hurricane } \\
\text { (USA) }\end{array}$ & $\begin{array}{l}\text { Cross- } \\
\text { sectional }\end{array}$ & Individual & $\begin{array}{l}98 \text { children } \\
\text { aged } 6-12 \\
\text { years } \\
147 \text { children } \\
\text { and } \\
\text { adolescents } \\
\text { aged 6-18 } \\
\text { years }\end{array}$ & $\begin{array}{l}\text { Age, } \\
\text { gender, } \\
\text { disaster } \\
\text { exposure }\end{array}$ & & Fears \\
\hline $\begin{array}{l}\text { Buthmann J, } \\
\text { Finik J, } \\
\text { Ventura G, } \\
\text { Zhang W, } \\
\text { Shereen AD, } \\
\text { Nomura Y. } \\
(2019)\end{array}$ & $\begin{array}{l}\text { Storm } \\
\text { (USA) }\end{array}$ & $\begin{array}{l}\text { Cross- } \\
\text { sectional }\end{array}$ & $\begin{array}{l}\text { Individual } \\
\text { Interpersona } \\
1\end{array}$ & $\begin{array}{l}198 \text { children, } \\
\text { 3-4 years old }\end{array}$ & $\begin{array}{l}\text { Maternal } \\
\text { prenatal } \\
\text { depression } \\
\text { Prenatal } \\
\text { exposure to } \\
\text { the disaster } \\
\text { Ethnicity } \\
\text { Maternal } \\
\text { anxiety and } \\
\text { PTSD } \\
\text { Maternal } \\
\text { education } \\
\text { and marital } \\
\text { status }\end{array}$ & $\begin{array}{l}4 \text { years after } \\
\text { storm }\end{array}$ & $\begin{array}{l}\text { Electroder } \\
\text { mal } \\
\text { activity }\end{array}$ \\
\hline $\begin{array}{l}\text { Buthmann, J., } \\
\text { Ham, J., } \\
\text { Davey, K., } \\
\text { Finik, J., } \\
\text { Dana, K., } \\
\text { Pehme, P., ... } \\
\text { Nomura, Y. } \\
\text { (2018) }\end{array}$ & $\begin{array}{l}\text { Storm } \\
\text { (USA) }\end{array}$ & $\begin{array}{l}\text { Cross- } \\
\text { sectional }\end{array}$ & $\begin{array}{l}\text { Individual } \\
\text { Interpersona } \\
1\end{array}$ & $\begin{array}{l}380 \text { pregnant } \\
\text { women } \\
380 \text { children, } \\
6 \text { months old }\end{array}$ & $\begin{array}{l}\text { Maternal } \\
\text { state anxiety, } \\
\text { stressful life } \\
\text { events, } \\
\text { prenatal } \\
\text { depression, } \\
\text { postnatal } \\
\text { depression, } \\
\text { the number } \\
\text { of birth }\end{array}$ & $\begin{array}{l}\text { before, during } \\
\text { and } 6 \text { months } \\
\text { after storm }\end{array}$ & $\begin{array}{l}\text { Child } \\
\text { Temperam } \\
\text { ent } \\
\text { (Negative } \\
\text { Affect, } \\
\text { Emotion } \\
\text { Regulation } \\
\text { ' } \\
\text { and } \\
\text { Surgency) }\end{array}$ \\
\hline
\end{tabular}




\section{RESILIENCE IN CHILDREN AFTER NATURAL DISASTERS}

\begin{tabular}{|c|c|c|c|c|c|c|c|c|c|}
\hline & & & & & $\begin{array}{l}\text { complication } \\
\text { s, maternal } \\
\text { age, parity, } \\
\text { education } \\
\text { attainment, } \\
\text { endocrine } \\
\text { disorders } \\
\text { and } \\
\text { infections. } \\
\text { Child } \\
\text { birthweight } \\
\text { gestational } \\
\text { age at birth, } \\
\text { sex } \\
\text { Objective } \\
\text { and } \\
\text { subjective } \\
\text { disaster- } \\
\text { related } \\
\text { stress, } \\
\text { and } \\
\text { gestational } \\
\text { timing of } \\
\text { exposure }\end{array}$ & & & & \\
\hline $\begin{array}{l}\text { Cadamuro, } \\
\text { A., Versari, } \\
\text { A., Vezzali, } \\
\text { L., \& } \\
\text { Trifiletti, E. } \\
\text { (2016). }\end{array}$ & $\begin{array}{l}\text { Earthquake } \\
\text { (Italy) }\end{array}$ & $\begin{array}{l}\text { Cross- } \\
\text { sectional }\end{array}$ & $\begin{array}{l}\text { Individual } \\
\text { and } \\
\text { Interpersona } \\
1\end{array}$ & $\begin{array}{l}147 \text { children, } \\
\text { aged 6-8 } \\
\text { years, } \\
294 \text { parent's }\end{array}$ & $\begin{array}{l}\text { Parent's } \\
\text { posttraumati } \\
\text { c stress } \\
\text { symptoms }\end{array}$ & $\begin{array}{l}\text { Children's } \\
\text { posttrauma } \\
\text { tic stress } \\
\text { symptoms }\end{array}$ & $\begin{array}{l}\text { ToM } \\
\text { Abilities }\end{array}$ & $\begin{array}{l}6 \text { months } \\
\text { post disaster }\end{array}$ & $\begin{array}{l}\text { Negative } \\
\text { Coping }\end{array}$ \\
\hline $\begin{array}{l}\text { Cadamuro, } \\
\text { A., Versari, } \\
\text { A., Vezzali, } \\
\text { L., } \\
\text { Giovannini, } \\
\text { D., \& } \\
\text { Trifiletti, E. } \\
(2015) .\end{array}$ & $\begin{array}{l}\text { Earthquake } \\
\text { (Italy) }\end{array}$ & $\begin{array}{l}\text { Cross- } \\
\text { sectional }\end{array}$ & $\begin{array}{l}\text { Individual } \\
\text { and } \\
\text { Interpersona } \\
1\end{array}$ & $\begin{array}{l}517 \\
\text { elementary } \\
\text { school } \\
\text { children } \\
\text { aged } 7-12 \\
\text { years }\end{array}$ & $\begin{array}{l}\text { Coping } \\
\text { styles }\end{array}$ & $\begin{array}{l}\text { Theory of } \\
\text { mind }\end{array}$ & $\begin{array}{l}\text { Social } \\
\text { support }\end{array}$ & $\begin{array}{l}6 \text { months } \\
\text { post disaster }\end{array}$ & $\begin{array}{l}\text { Cognitive } \\
\text { Performan } \\
\text { ce }\end{array}$ \\
\hline $\begin{array}{l}\text { Cao, X., } \\
\text { Laplante, D. }\end{array}$ & Ice Storm & $\begin{array}{l}\text { Cross- } \\
\text { sectional }\end{array}$ & $\begin{array}{l}\text { Individual } \\
\text { and }\end{array}$ & 89 children & $\begin{array}{l}\text { Maternal } \\
\text { distress, }\end{array}$ & & & $\begin{array}{l}5 \text { years } \\
\text { post disaster }\end{array}$ & $\begin{array}{l}\text { Neuromoto } \\
\mathrm{r} \text { function }\end{array}$ \\
\hline
\end{tabular}




\begin{tabular}{|c|c|c|c|c|c|c|c|c|}
\hline $\begin{array}{l}\text { P., Brunet, A., } \\
\text { Ciampi, A., \& } \\
\text { King, S. } \\
(2014) .\end{array}$ & (Canada) & & $\begin{array}{l}\text { Interpersona } \\
1\end{array}$ & $\begin{array}{l}\text { age } \mathrm{M}=5,5 \\
\text { years } \\
\text { and } \\
\text { their } \\
\text { mothers }\end{array}$ & $\begin{array}{l}\text { timing of } \\
\text { exposure, } \\
\text { level of } \\
\text { exposure, } \\
\text { major life } \\
\text { events, } \\
\text { gender, } \\
\text { trimester of } \\
\text { pregnancy, } \\
\text { socioeconom } \\
\text { ic status }\end{array}$ & & & \\
\hline $\begin{array}{l}\text { Catani, C., } \\
\text { Gewirtz, A. } \\
\text { H., Wieling, } \\
\text { E., Schauer, } \\
\text { E., Elbert, T., } \\
\text { \& Neuner, F. } \\
(2010) .\end{array}$ & $\begin{array}{l}\text { Tsunami, } \\
\text { War and } \\
\text { Family } \\
\text { Violence } \\
\text { (4 Studies) } \\
\text { (Sri Lanka) }\end{array}$ & $\begin{array}{l}\text { Cross- } \\
\text { sectional }\end{array}$ & Individual & $\begin{array}{l}1398 \\
\text { children } \\
\text { aged 9-15 } \\
\text { years }\end{array}$ & $\begin{array}{l}\text { Severity of } \\
\text { disaster } \\
\text { exposure, } \\
\text { economic } \\
\text { status, } \\
\text { family loss, } \\
\text { war } \\
\text { experiences, } \\
\text { family } \\
\text { violence, } \\
\text { exposure to } \\
\text { other } \\
\text { traumatic } \\
\text { experiences, } \\
\text { multiplicity } \\
\text { of risks }\end{array}$ & & $\begin{array}{l}1 \text { month } \\
\text { post disaster } \\
\text { (study } 3 \text { ) } \\
\text { and } \\
12 \text { months } \\
\text { post disaster } \\
\text { (study } 4 \text { ) }\end{array}$ & $\begin{array}{l}\text { Adaptation } \\
\text { score } \\
\text { (Physical } \\
\text { Health, } \\
\text { Functionin } \\
\text { g, Mental } \\
\text { Health, } \\
\text { School } \\
\text { performan } \\
\text { ce) }\end{array}$ \\
\hline $\begin{array}{l}\text { Celebi Oncu, } \\
\text { E., \& } \\
\text { Metindogan } \\
\text { Wise, A. } \\
(2010) .\end{array}$ & $\begin{array}{l}\text { Earthquake } \\
\text { (Turkey) }\end{array}$ & $\begin{array}{l}\text { Experiment } \\
\text { al (with } \\
\text { control } \\
\text { group) }\end{array}$ & Individual & $\begin{array}{l}103 \text { children } \\
\text { aged } 9 \text { years }\end{array}$ & $\begin{array}{l}\text { Disaster } \\
\text { exposure, } \\
\text { group, }\end{array}$ & Gender & $\begin{array}{l}20 \text { months } \\
\text { post disaster }\end{array}$ & $\begin{array}{l}\text { Trauma- } \\
\text { related } \\
\text { symptoms }\end{array}$ \\
\hline $\begin{array}{l}\text { Celebi Oncu, } \\
\text { E., Akman, } \\
\text { B., Guler, T., } \\
\text { \& Karaaslan, } \\
\text { T. (2009). }\end{array}$ & $\begin{array}{l}\text { Earthquake } \\
\text { (Turkey) }\end{array}$ & $\begin{array}{l}\text { Cross- } \\
\text { Sectional }\end{array}$ & Individual & $\begin{array}{l}64 \text { children } \\
\text { aged } 6 \text { years }\end{array}$ & $\begin{array}{l}\text { Disaster } \\
\text { exposure }\end{array}$ & & & $\begin{array}{l}\text { Emotions } \\
\text { in } \\
\text { drawings }\end{array}$ \\
\hline $\begin{array}{l}\text { Cénat, J. M., } \\
\& \text { Derivois, } \\
\text { D. }(2015) .\end{array}$ & $\begin{array}{l}\text { Earthquake } \\
\text { (Haiti) }\end{array}$ & $\begin{array}{l}\text { Cross- } \\
\text { sectional }\end{array}$ & Individual & $\begin{array}{l}872 \text { children } \\
\text { aged } 7-17 \\
\text { years }\end{array}$ & $\begin{array}{l}\text { Peritraumati } \\
\text { c distress, }\end{array}$ & & & $\begin{array}{l}\text { PTSD } \\
\text { Depression }\end{array}$ \\
\hline
\end{tabular}




\begin{tabular}{|c|c|c|c|c|c|c|c|c|}
\hline & & & & & $\begin{array}{l}\text { disaster } \\
\text { exposure, } \\
\text { socio- } \\
\text { demographic } \\
\text { characteristi } \\
\text { cs }\end{array}$ & & & \\
\hline $\begin{array}{l}\text { Chen, J., \& } \\
\text { Wu, X. } \\
(2017) .\end{array}$ & $\begin{array}{l}\text { Earthquake } \\
\text { (China) }\end{array}$ & $\begin{array}{l}\text { Longitudin } \\
\text { al }\end{array}$ & Individual & $\begin{array}{l}757 \text { children } \\
9-16 \text { years } \\
\text { old }\end{array}$ & $\begin{array}{l}\text { Gender } \\
\text { Age } \\
\text { Educational } \\
\text { level } \\
\text { Disaster } \\
\text { Exposure }\end{array}$ & & $\begin{array}{l}\text { between } 8 \text { and } \\
20 \text { months } \\
\text { after the } \\
\text { earthquake }\end{array}$ & $\begin{array}{l}\text { Posttrauma } \\
\text { tic stress } \\
\text { disorder } \\
\text { (PTSD) } \\
\text { and } \\
\text { posttrauma } \\
\text { tic growth } \\
\text { (PTG) }\end{array}$ \\
\hline $\begin{array}{l}\text { Chen, W., } \\
\text { Wang, L., } \\
\text { Zhang, X. L., } \\
\text { \& Shi, J. N. } \\
\text { (2012). }\end{array}$ & $\begin{array}{l}\text { Earthquake } \\
\text { (China) }\end{array}$ & $\begin{array}{l}\text { Cross- } \\
\text { sectional }\end{array}$ & Individual & $\begin{array}{l}156 \text { children } \\
\text { age } M=11 \\
\text { years }\end{array}$ & $\begin{array}{l}\text { Disaster } \\
\text { exposure, } \\
\text { emotional } \\
\text { focus, } \\
\text { coping } \\
\text { strategy }\end{array}$ & $\begin{array}{l}\text { Emotional } \\
\text { coping } \\
\text { strategy } \\
\text { Self- } \\
\text { esteem }\end{array}$ & $\begin{array}{l}6 \text { months } \\
\text { post disaster }\end{array}$ & PTSS \\
\hline $\begin{array}{l}\text { Cheng, J., } \\
\text { Liang, Y., Fu, } \\
\text { L. \& Liu, Z. } \\
(2018)\end{array}$ & $\begin{array}{l}\text { Earthquake } \\
\text { (China) }\end{array}$ & $\begin{array}{l}\text { Longitudin } \\
\text { al }\end{array}$ & $\begin{array}{l}\text { Individual } \\
\text { and } \\
\text { Interpersona } \\
1\end{array}$ & $\begin{array}{l}301 \text { children } \\
\text { aged } 9.6- \\
14.6 \text { years }\end{array}$ & $\begin{array}{l}\text { Disaster } \\
\text { exposure, } \\
\text { gender, } \\
\text { grade, } \\
\text { parental } \\
\text { relationship, } \\
\text { PTSD and } \\
\text { depression } \\
(\mathrm{T} 1, \mathrm{~T} 2, \mathrm{~T} 3)\end{array}$ & & $\begin{array}{l}\text { 4-29 months } \\
\text { post disaster } \\
(\mathrm{T} 1-\mathrm{T} 2) \\
40-52 \text { months } \\
\text { post disaster }\end{array}$ & $\begin{array}{l}\text { PTSD } \\
\text { Depression } \\
\text { and } \\
\text { comorbilit } \\
\text { y }\end{array}$ \\
\hline $\begin{array}{l}\text { Cheng, J., } \\
\text { Liang, Y.-M., } \\
\text { Zhou, Y.-Y., } \\
\text { Eli, B., \& Liu, } \\
\text { Z.-K. (2019) }\end{array}$ & $\begin{array}{l}\text { Earthquake } \\
\text { (China) }\end{array}$ & $\begin{array}{l}\text { Longitudin } \\
\text { al }\end{array}$ & $\begin{array}{l}\text { Individual } \\
\text { Interpersona } \\
1\end{array}$ & $\begin{array}{l}304 \\
\text { children, 9- } \\
17 \text { years old }\end{array}$ & $\begin{array}{l}\text { Trauma } \\
\text { exposure } \\
\text { Quality } \\
\text { Parental } \\
\text { relationships } \\
\text { Grade } \\
\text { Gender } \\
\text { Social } \\
\text { support }\end{array}$ & & $\begin{array}{l}1.5,6, \\
12,24 \text { and } 48 \\
\text { months after } \\
\text { the earthquake }\end{array}$ & PTSD \\
\hline
\end{tabular}




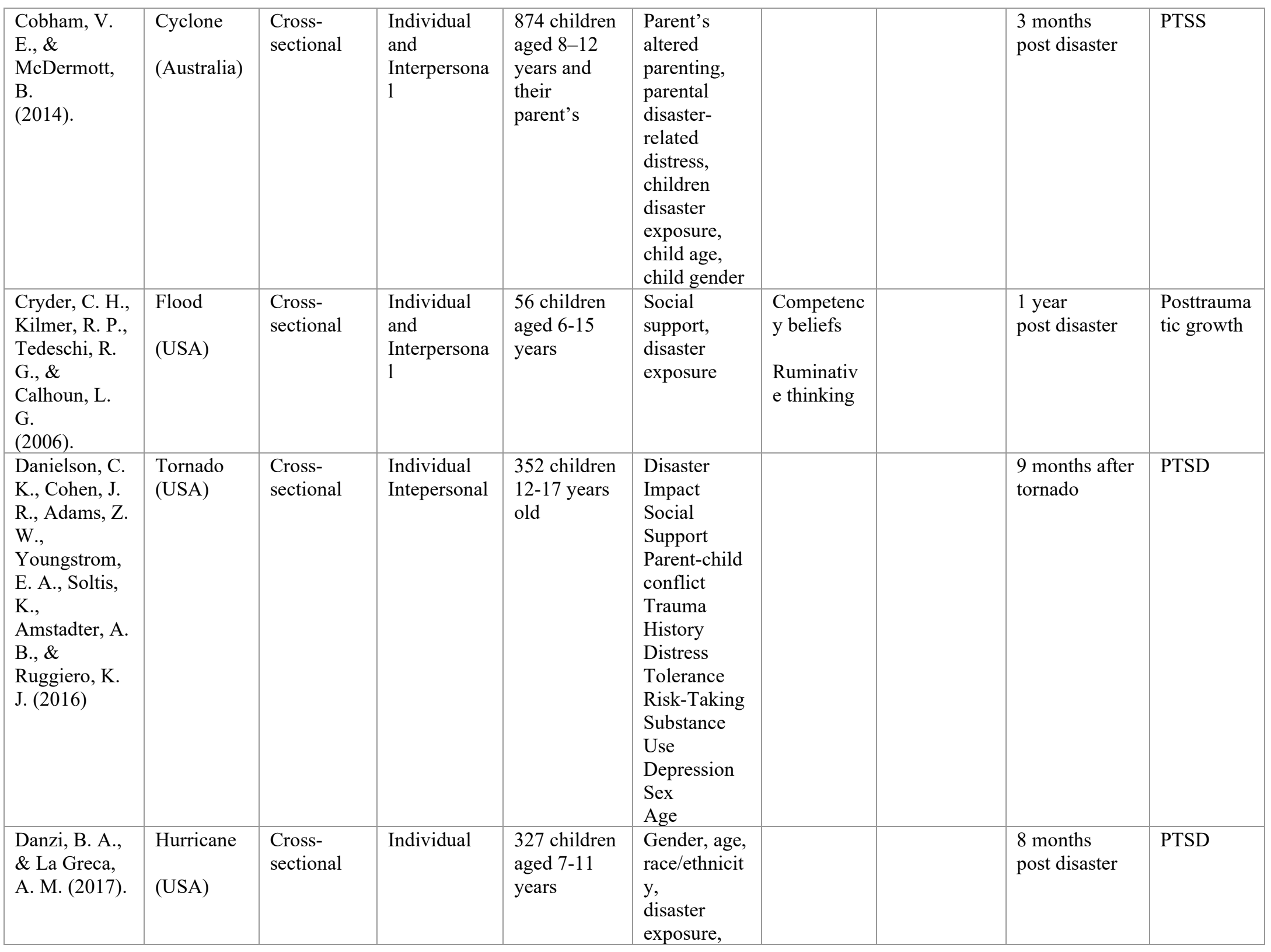




\begin{tabular}{|c|c|c|c|c|c|c|c|}
\hline & & & & & $\begin{array}{l}\text { hurricane } \\
\text { related life } \\
\text { stressors, } \\
\text { impairment }\end{array}$ & & \\
\hline $\begin{array}{l}\text { Dass- } \\
\text { Brailsford, P., } \\
\text { Thomley, R. } \\
\text { S. H., } \\
\text { Talisman, N. } \\
\text { W., \& } \\
\text { Unverferth, } \\
\text { K. (2015). }\end{array}$ & $\begin{array}{l}\text { Earthquake } \\
\text { (Haiti) }\end{array}$ & $\begin{array}{l}\text { Cross- } \\
\text { sectional }\end{array}$ & Individual & $\begin{array}{l}59 \text { children } \\
\text { aged 9-12 } \\
\text { years }\end{array}$ & $\begin{array}{l}\text { Distance } \\
\text { from the } \\
\text { epicenter } \\
\text { (urban vs. } \\
\text { rural), } \\
\text { children } \\
\text { displayed or } \\
\text { not }\end{array}$ & $\begin{array}{l}12 \text { months } \\
\text { post disaster }\end{array}$ & $\begin{array}{l}\text { Psychologi } \\
\text { cal } \\
\text { consequen } \\
\text { ces } \\
\text { (depressio } \\
\text { n, } \\
\text { anxiety, } \\
\text { and } \\
\text { trauma) }\end{array}$ \\
\hline $\begin{array}{l}\text { Dawson, K. } \\
\text { S., Joscelyne, } \\
\text { A., Meijer, C., } \\
\text { Tampubolon, } \\
\text { A., Steel, Z., } \\
\text { \& Bryant, R. } \\
\text { A. (2014). }\end{array}$ & $\begin{array}{l}\text { Tsunami } \\
\text { (Indonesia) }\end{array}$ & $\begin{array}{l}\text { Cross- } \\
\text { sectional }\end{array}$ & Individual & $\begin{array}{l}110 \text { children } \\
\text { aged } 7-13 \\
\text { years }\end{array}$ & $\begin{array}{l}\text { Religious } \\
\text { belief, } \\
\text { disaster } \\
\text { exposure, } \\
\text { cognitive } \\
\text { avoidance, } \\
\text { age }\end{array}$ & $\begin{array}{l}5 \text { years } \\
\text { post disaster }\end{array}$ & $\begin{array}{l}\text { PTSD, } \\
\text { depression, } \\
\text { grief }\end{array}$ \\
\hline $\begin{array}{l}\text { Delamater, A. } \\
\text { M., \& } \\
\text { Applegate, E. } \\
\text { B. (1999). }\end{array}$ & $\begin{array}{l}\text { Hurricane } \\
\text { (USA) }\end{array}$ & $\begin{array}{l}\text { Longitudin } \\
\text { al }\end{array}$ & Individual & $\begin{array}{l}175 \text { children } \\
\text { aged } 3-5 \\
\text { years }\end{array}$ & $\begin{array}{l}\text { Disaster } \\
\text { exposure, } \\
\text { PTSD, age, } \\
\text { gender, } \\
\text { ethnic status, } \\
\text { marital } \\
\text { status, and } \\
\text { highest } \\
\text { grade } \\
\text { completed } \\
\text { by mothers }\end{array}$ & $\begin{array}{l}12 \text { and } 18 \\
\text { months post } \\
\text { disaster }\end{array}$ & $\begin{array}{l}\text { Child } \\
\text { Developm } \\
\text { ent }\end{array}$ \\
\hline
\end{tabular}




\begin{tabular}{|c|c|c|c|c|c|c|c|}
\hline $\begin{array}{l}\text { \& Kayaalp, L. } \\
(2010) .\end{array}$ & & & & & $\begin{array}{l}\text { was } \\
\text { experience, } \\
\text { degree of } \\
\text { losses, } \\
\text { bodily } \\
\text { injuries, } \\
\text { damage to } \\
\text { the residence }\end{array}$ & & \\
\hline $\begin{array}{l}\text { Derivois, D., } \\
\text { Cénat, J. M., } \\
\text { Joseph, N. E., } \\
\text { Karray, A., \& } \\
\text { Chahraoui, K. } \\
(2017) .\end{array}$ & $\begin{array}{l}\text { Earthquake } \\
\text { (Haiti) }\end{array}$ & $\begin{array}{l}\text { Cross- } \\
\text { sectional }\end{array}$ & Individual & $\begin{array}{l}128 \text { children } \\
\text { and } \\
\text { adolescents } \\
\text { aged } 7-18 \\
\text { years (living } \\
\text { on the } \\
\text { streets) }\end{array}$ & $\begin{array}{l}\text { Age, gender, } \\
\text { disaster } \\
\text { exposure, } \\
\text { life events, } \\
\text { peritraumati } \\
\text { c distress, } \\
\text { reason for } \\
\text { living in the } \\
\text { street }\end{array}$ & $\begin{array}{l}4 \text { years } \\
\text { post disaster }\end{array}$ & $\begin{array}{l}\text { PTSD, } \\
\text { anxiety } \\
\text { depression }\end{array}$ \\
\hline $\begin{array}{l}\text { Derivois, D., } \\
\text { Mérisier, G. } \\
\text { G., Cenat, J. } \\
\text { M., \& } \\
\text { Castelot, V. } \\
(2014) .\end{array}$ & $\begin{array}{l}\text { Earthquake } \\
\text { (Haiti) }\end{array}$ & $\begin{array}{l}\text { Cross- } \\
\text { sectional }\end{array}$ & $\begin{array}{l}\text { Individual } \\
\text { and } \\
\text { Interpersona } \\
1\end{array}$ & $\begin{array}{l}540 \text { children } \\
\text { and } \\
\text { adolescents } \\
\text { aged } 2-18 \\
\text { years }\end{array}$ & $\begin{array}{l}\text { Age, gender, } \\
\text { level of } \\
\text { education, } \\
\text { religion, } \\
\text { health } \\
\text { conditions, } \\
\text { trauma } \\
\text { exposure, } \\
\text { others } \\
\text { traumatic } \\
\text { events, } \\
\text { post- } \\
\text { earthquake } \\
\text { situation and } \\
\text { the } \\
\text { peritraumati } \\
\text { c distress, } \\
\text { social } \\
\text { support }\end{array}$ & $\begin{array}{l}16 \text { to } 18 \\
\text { months } \\
\text { post disaster }\end{array}$ & PTSD \\
\hline
\end{tabular}




\begin{tabular}{|c|c|c|c|c|c|c|c|}
\hline & & & & & & $\begin{array}{l}9 \text { months post } \\
\text { incident (T2) }\end{array}$ & $\begin{array}{l}\text { Adjustmen } \\
\mathrm{t} \text { reactions } \\
\text { Sleep } \\
\text { disturbanc } \\
\text { e. }\end{array}$ \\
\hline $\begin{array}{l}\text { Dollinger, S. } \\
\text { J. (1986). }\end{array}$ & $\begin{array}{l}\text { Lightning- } \\
\text { Strike } \\
\text { (USA) }\end{array}$ & $\begin{array}{l}\text { Cross- } \\
\text { sectional }\end{array}$ & Individual & $\begin{array}{l}38 \text { children } \\
\text { aged } 10-12 \\
\text { years }\end{array}$ & $\begin{array}{l}\text { Disaster } \\
\text { exposure, } \\
\text { fears of } \\
\text { storms, } \\
\text { death and } \\
\text { dying, } \\
\text { separation } \\
\text { from parents }\end{array}$ & $\begin{array}{l}1-2 \\
\text { months post } \\
\text { incident }\end{array}$ & $\begin{array}{l}\text { Sleep } \\
\text { disturbanc } \\
\text { es and } \\
\text { somatic } \\
\text { complaints }\end{array}$ \\
\hline $\begin{array}{l}\text { Dollinger, S. } \\
\text { J., O'donnell, } \\
\text { J. P., \& } \\
\text { Staley, A. A. } \\
(1984) \text {. }\end{array}$ & $\begin{array}{l}\text { Lightning- } \\
\text { Strike } \\
\text { (USA) }\end{array}$ & $\begin{array}{l}\text { Cross- } \\
\text { sectional }\end{array}$ & $\begin{array}{l}\text { Individual } \\
\text { and } \\
\text { Interpersona } \\
1\end{array}$ & $\begin{array}{l}87 \text { children } \\
\text { aged } 10-13 \\
\text { years }\end{array}$ & $\begin{array}{l}\text { Age, gender, } \\
\text { socio } \\
\text { economics } \\
\text { status, } \\
\text { disaster } \\
\text { exposure, } \\
\text { fears of } \\
\text { storms, } \\
\text { separation } \\
\text { from } \\
\text { parents, } \\
\text { death and } \\
\text { dying }\end{array}$ & $\begin{array}{l}1-2 \text { months } \\
\text { post incident }\end{array}$ & $\begin{array}{l}\text { Sleep } \\
\text { disturbanc } \\
\text { es and } \\
\text { somatic } \\
\text { complaints }\end{array}$ \\
\hline $\begin{array}{l}\text { Durkin, M. S., } \\
\text { Khan, N., } \\
\text { Davidson, L. } \\
\text { L., Zaman, S. } \\
\text { S., \& Stein, Z. } \\
\text { A. (1993). }\end{array}$ & $\begin{array}{l}\text { Flood } \\
\text { (Banglades } \\
\text { h) }\end{array}$ & $\begin{array}{l}\text { Pre post } \\
\text { (without } \\
\text { control } \\
\text { group) }\end{array}$ & Individual & $\begin{array}{l}\begin{array}{l}2667 \\
\text { children } \\
\text { aged 2-9 } \\
\text { years, (pre } \\
\text { disaster) }\end{array} \\
162 \text { children } \\
\text { (post } \\
\text { disaster) }\end{array}$ & $\begin{array}{l}\text { Disaster } \\
\text { exposure }\end{array}$ & $\begin{array}{l}6 \text { months } \\
\text { pre disaster } \\
5 \text { months } \\
\text { post disaster }\end{array}$ & $\begin{array}{l}\text { Behaviour } \\
\text { al } \\
\text { disorders } \\
\text { in children }\end{array}$ \\
\hline
\end{tabular}




\begin{tabular}{|c|c|c|c|c|c|c|c|c|}
\hline $\begin{array}{l}\text { Dyb, G., } \\
\text { Jensen, T. K., } \\
\text { \& Nygaard, E. } \\
(2011) .\end{array}$ & $\begin{array}{l}\text { Tsunami } \\
\text { (Southeast } \\
\text { Asia) }\end{array}$ & $\begin{array}{l}\text { Cross- } \\
\text { sectional }\end{array}$ & $\begin{array}{l}\text { Individual } \\
\text { and } \\
\text { Interpersona } \\
1\end{array}$ & $\begin{array}{l}319 \text { parents } \\
\text { and } \\
\text { adolescents } \\
\text { aged } 6-18 \\
\text { years }\end{array}$ & $\begin{array}{l}\text { Disaster } \\
\text { exposure } \\
\text { (parents and } \\
\text { child), } \\
\text { parent's } \\
\text { level of } \\
\text { posttraumati } \\
\text { c stress }\end{array}$ & $\begin{array}{l}\text { Parental } \\
\text { PTSD }\end{array}$ & $\begin{array}{l}6-8 \text { months } \\
\text { post } \\
\text { tsunami }\end{array}$ & PTSD \\
\hline $\begin{array}{l}\text { Earls, F., } \\
\text { Smith, E., } \\
\text { Reich, W., \& } \\
\text { Jung, K. G. } \\
\text { (1988). }\end{array}$ & $\begin{array}{l}\text { Flood } \\
\text { (USA) }\end{array}$ & $\begin{array}{l}\text { Cross- } \\
\text { sectional }\end{array}$ & $\begin{array}{l}\text { Individual } \\
\text { and } \\
\text { Interpersona } \\
1\end{array}$ & $\begin{array}{l}\text { Parents and } \\
32 \text { children } \\
\text { and } \\
\text { adolescent } \\
\text { aged 6-17 } \\
\text { years }\end{array}$ & $\begin{array}{l}\text { Disaster } \\
\text { exposure, } \\
\text { preexisting } \\
\text { psychiatric } \\
\text { disorders, } \\
\text { parent's } \\
\text { reactions to } \\
\text { the flood }\end{array}$ & & $\begin{array}{l}12 \text { months } \\
\text { post disaster }\end{array}$ & PTSS \\
\hline
\end{tabular}




\begin{tabular}{|c|c|c|c|c|c|c|c|}
\hline & & & & & $\begin{array}{l}\text { injured, } \\
\text { family } \\
\text { member } \\
\text { injured, lost } \\
\text { (3) post- } \\
\text { disaster } \\
\text { response } \\
\text { factors: } \\
\text { reaction, } \\
\text { feelings, } \\
\text { relocation }\end{array}$ & & \\
\hline $\begin{array}{l}\text { Endo, T., } \\
\text { Shioiri, T., } \\
\text { Someya, T., } \\
\text { Toyabe, S., \& } \\
\text { Akazawa, K. } \\
\text { (2007). }\end{array}$ & $\begin{array}{l}\text { Earthquake } \\
\text { (Japan) }\end{array}$ & $\begin{array}{l}\text { Cross- } \\
\text { sectional }\end{array}$ & $\begin{array}{l}\text { Individual } \\
\text { and } \\
\text { Interpersona } \\
1\end{array}$ & $\begin{array}{l}756 \text { children } \\
\text { including } \\
170 \text { pre- } \\
\text { school } \\
(<7 \text { years } \\
\text { old) and } \\
586 \\
\text { school- } \\
\text { children ( }>7 \\
\text { years old) }\end{array}$ & $\begin{array}{l}\text { Demographi } \\
\text { c } \\
\text { information, } \\
\text { parents and } \\
\text { children's } \\
\text { physical } \\
\text { state after } \\
\text { the } \\
\text { earthquake } \\
\text { (injury and } \\
\text { illness), } \\
\text { damage to } \\
\text { house }\end{array}$ & $\begin{array}{l}5 \text { months post } \\
\text { disaster }\end{array}$ & $\begin{array}{l}\text { Behavioral } \\
\text { changes in } \\
\text { children }\end{array}$ \\
\hline $\begin{array}{l}\text { Evans, L. G., } \\
\text { \& Oehler- } \\
\text { Stinnett, J. } \\
\text { (2006). }\end{array}$ & $\begin{array}{l}\text { Tornado } \\
\text { (USA) }\end{array}$ & $\begin{array}{l}\text { Cross- } \\
\text { sectional }\end{array}$ & Individual & $\begin{array}{l}152 \text { children } \\
\text { aged } 6-12 \\
\text { years }\end{array}$ & $\begin{array}{l}\text { Age, gender, } \\
\text { ethnicity, } \\
\text { disaster } \\
\text { exposure }\end{array}$ & $\begin{array}{l}\text { 1-year } \\
\text { post disaster }\end{array}$ & PTSD \\
\hline $\begin{array}{l}\text { Evans, L. G., } \\
\text { \& Oehler- } \\
\text { Stinnett, J. } \\
(2008) .\end{array}$ & $\begin{array}{l}\text { Tornado } \\
\text { (USA) }\end{array}$ & $\begin{array}{l}\text { Cross- } \\
\text { sectional }\end{array}$ & Individual & $\begin{array}{l}88 \text { children } \\
\text { aged } 7-12 \\
\text { years }\end{array}$ & $\begin{array}{l}\text { Age, gender, } \\
\text { ethnicity, } \\
\text { disaster } \\
\text { exposure, } \\
\text { PTSD }\end{array}$ & & $\begin{array}{l}\text { PTSD } \\
\text { Internalizi } \\
\text { ng } \\
\text { symptoms } \\
\text { and } \\
\text { adjustment }\end{array}$ \\
\hline $\begin{array}{l}\text { Exenberger, } \\
\text { S., } \\
\text { Ramalingam, } \\
\text { P., \& Höfer, } \\
\text { S. (2016). }\end{array}$ & $\begin{array}{l}\text { Tsunami } \\
\text { (Southeast } \\
\text { Asia) }\end{array}$ & $\begin{array}{l}\text { Cross- } \\
\text { sectional }\end{array}$ & Individual & $\begin{array}{l}177 \text { South } \\
\text { Indian } \\
\text { children } \\
\text { aged } 8-17 \\
\text { years }\end{array}$ & $\begin{array}{l}\text { PTSS, } \\
\text { growth, } \\
\text { gender, age }\end{array}$ & $\begin{array}{l}4 \text { years } \\
\text { post disaster }\end{array}$ & $\begin{array}{l}\text { Posttrauma } \\
\text { tic Growth } \\
\text { (PTG) } \\
\text { PTSD }\end{array}$ \\
\hline
\end{tabular}




\section{RESILIENCE IN CHILDREN AFTER NATURAL DISASTERS}

\begin{tabular}{|c|c|c|c|c|c|c|c|c|}
\hline $\begin{array}{l}\text { Exenberger, } \\
\text { S., Riedl, D., } \\
\text { Rangaramanuj } \\
\text { am, K., } \\
\text { Amirtharaj, } \\
\text { V., \& Juen, F. } \\
\text { (2019) }\end{array}$ & $\begin{array}{l}\text { Tsunami } \\
\text { (India) }\end{array}$ & $\begin{array}{l}\text { Cross- } \\
\text { sectional }\end{array}$ & $\begin{array}{l}\text { Individual } \\
\text { Intepersonal }\end{array}$ & $\begin{array}{l}80 \text { mothers } \\
\text { and } 164 \\
\text { children } 8 \text { - } \\
17 \text { years old }\end{array}$ & $\begin{array}{l}\text { mother's } \\
\text { PTSD } \\
\text { symptoms } \\
\text { living } \\
\text { arrangement } \\
\text { age, gender } \\
\text { religion }\end{array}$ & & $\begin{array}{l}4 \text { years post } \\
\text { tsunami }\end{array}$ & PTSD \\
\hline $\begin{array}{l}\text { Fan, F., Long, } \\
\text { K., Zhou, Y., } \\
\text { Zheng, Y., \& } \\
\text { Liu, X. } \\
(2015) .\end{array}$ & $\begin{array}{l}\text { Earthquake } \\
\text { (China) }\end{array}$ & $\begin{array}{l}\text { Longitudin } \\
\text { al }\end{array}$ & $\begin{array}{l}\text { Individual } \\
\text { and } \\
\text { Interpersona } \\
1\end{array}$ & $\begin{array}{l}1573 \\
\text { adolescents } \\
\text { aged 12-16 } \\
\text { years }\end{array}$ & $\begin{array}{l}\text { Demographi } \\
\text { cs, disaster } \\
\text { exposure, } \\
\text { exposure, } \\
\text { gender, age, } \\
\text { severity } \\
\text { of negative } \\
\text { life events, } \\
\text { social } \\
\text { support, } \\
\text { coping style }\end{array}$ & & $\begin{array}{l}6 \text { months post } \\
\text { disaster } \\
\text { (T1) } \\
12 \text { months } \\
\text { post disaster } \\
\text { (T2) } \\
24 \text { months } \\
\text { post disaster } \\
\text { (T3) }\end{array}$ & PTSD \\
\hline $\begin{array}{l}\text { Felix, E. D., } \\
\text { You, S. K., \& } \\
\text { Canino, G. } \\
(2013) .\end{array}$ & $\begin{array}{l}\text { Hurricane } \\
\text { (Puerto } \\
\text { Rico) }\end{array}$ & $\begin{array}{l}\text { Cross- } \\
\text { sectional }\end{array}$ & $\begin{array}{l}\text { Individual } \\
\text { and } \\
\text { Interpersona } \\
1\end{array}$ & $\begin{array}{l}1637 \\
\text { children and } \\
\text { adolescents } \\
\text { aged } 6-17 \\
\text { years and } \\
\text { parents }\end{array}$ & $\begin{array}{l}3 \text { age } \\
\text { groups, } \\
\text { gender, } \\
\text { perception } \\
\text { of poverty, } \\
\text { disaster } \\
\text { exposure, } \\
\text { school } \\
\text { violence, } \\
\text { teacher's } \\
\text { attitudes, } \\
\text { neighborhoo } \\
\text { d climate, } \\
\text { neighborhoo } \\
\text { d } \\
\text { monitoring, } \\
\text { community } \\
\text { violence, } \\
\text { violence } \\
\text { exposure }\end{array}$ & $\begin{array}{l}\text { School } \\
\text { microsystem } \\
\text { s (violence), } \\
\text { Poor teacher } \\
\text { attitudes, } \\
\text { Community } \\
\text { characteristi } \\
\text { cs } \\
\text { (neighborho } \\
\text { od climate) }\end{array}$ & $\begin{array}{l}12-27 \text { months } \\
\text { post disaster }\end{array}$ & $\begin{array}{l}\text { Internalizi } \\
\text { ng } \\
\text { psychopath } \\
\text { ology }\end{array}$ \\
\hline
\end{tabular}




\begin{tabular}{|c|c|c|c|c|c|c|c|c|c|}
\hline $\begin{array}{l}\text { Felix, E. D., } \\
\text { You, S., \& } \\
\text { Canino, G. } \\
(2015)\end{array}$ & $\begin{array}{l}\text { Hurricane } \\
\text { (Puerto } \\
\text { Rico) }\end{array}$ & $\begin{array}{l}\text { Cross- } \\
\text { sectional }\end{array}$ & $\begin{array}{l}\text { Individual } \\
\text { and } \\
\text { Interpersona } \\
1\end{array}$ & $\begin{array}{l}582 \text { children } \\
\text { aged 4-10 } \\
\text { years } \\
\text { and } \\
\text { adolescent } \\
\text { aged 4-17 } \\
\text { years }\end{array}$ & $\begin{array}{l}\text { Family } \\
\text { environment } \\
\text { disaster } \\
\text { exposure, } \\
\text { parent-child } \\
\text { relationship } \\
\text { quality, age }\end{array}$ & $\begin{array}{l}\text { Family } \\
\text { environme } \\
\mathrm{nt} \\
\text { Parent } \\
\text { relationshi } \\
\text { p quality } \\
\text { (mediated } \\
\text { for } \\
\text { adolescent } \\
\text { s) }\end{array}$ & & $\begin{array}{l}18 \text { months } \\
\text { post disaster }\end{array}$ & $\begin{array}{l}\text { Ataques de } \\
\text { nervios }\end{array}$ \\
\hline $\begin{array}{l}\text { Felix, E., } \\
\text { Hernández, L. } \\
\text { A., Bravo, M., } \\
\text { Ramirez, R., } \\
\text { Cabiya, J., \& } \\
\text { Canino, G. } \\
(2011) .\end{array}$ & $\begin{array}{l}\text { Hurricane } \\
\text { (Puerto } \\
\text { Rico) }\end{array}$ & $\begin{array}{l}\text { Longitudin } \\
\text { al }\end{array}$ & Individual & $\begin{array}{l}1886 \\
\text { children } \\
(\mathrm{T} 1) \\
1788 \\
\text { children } \\
(\mathrm{T} 2) \\
\text { aged 4-17 } \\
\text { years }\end{array}$ & $\begin{array}{l}\text { Disaster } \\
\text { exposure, } \\
\text { gender }\end{array}$ & & Age & $\begin{array}{l}18-30 \text { months } \\
\text { post disaster }\end{array}$ & $\begin{array}{l}\text { Internalizi } \\
\text { ng, } \\
\text { externalizi } \\
\text { ng }\end{array}$ \\
\hline $\begin{array}{l}\text { Felix, E., } \\
\text { Kaniasty, K., } \\
\text { You, S., \& } \\
\text { Canino, G. } \\
(2015) .\end{array}$ & $\begin{array}{l}\text { Hurricane } \\
\text { (Puerto } \\
\text { Rico) }\end{array}$ & $\begin{array}{l}\text { Cross- } \\
\text { sectional }\end{array}$ & $\begin{array}{l}\text { Individual } \\
\text { and } \\
\text { Interpersona } \\
1\end{array}$ & $\begin{array}{l}1886 \text { parent- } \\
\text { child dyads } \\
\text { aged } 4-17 \\
\text { years }\end{array}$ & $\begin{array}{l}\text { economic } \\
\text { status, age, } \\
\text { exposure } \\
\text { time, parent- } \\
\text { child, } \\
\text { relationship } \\
\text { quality }\end{array}$ & & $\begin{array}{l}\text { Parent-Child } \\
\text { Relationship } \\
\text { Quality }\end{array}$ & $\begin{array}{l}18-30 \text { months } \\
\text { post disaster }\end{array}$ & $\begin{array}{l}\text { Physical } \\
\text { health }\end{array}$ \\
\hline $\begin{array}{l}\text { Felix, E., } \\
\text { You, S., } \\
\text { Vernberg, E., } \\
\text { \& Canino, G. } \\
(2013) .\end{array}$ & $\begin{array}{l}\text { Hurricane } \\
\text { (Puerto } \\
\text { Rico) }\end{array}$ & $\begin{array}{l}\text { Cross- } \\
\text { sectional }\end{array}$ & $\begin{array}{l}\text { Individual } \\
\text { and } \\
\text { Interpersona } \\
1\end{array}$ & $\begin{array}{l}1886 \text { dyads } \\
\text { Two ages } \\
\text { groups: } \\
4-10 \\
\text { and } \\
11-17\end{array}$ & $\begin{array}{l}\text { Disaster } \\
\text { exposure, } \\
\text { age }\end{array}$ & $\begin{array}{l}\text { Family } \\
\text { Environme } \\
\text { nt., } \\
\text { Parent- } \\
\text { child } \\
\text { relationshi } \\
\text { p quality }\end{array}$ & $\begin{array}{l}\text { Parental } \\
\text { history of } \\
\text { mental } \\
\text { health } \\
\text { problem }\end{array}$ & $\begin{array}{l}18 \text { months } \\
\text { post disaster }\end{array}$ & $\begin{array}{l}\text { Psychopat } \\
\text { hology:inte } \\
\text { rnalizing } \\
\text { disorder in } \\
\text { children }\end{array}$ \\
\hline
\end{tabular}




\begin{tabular}{|c|c|c|c|c|c|c|c|c|c|}
\hline $\begin{array}{l}\text { Felton, J. W., } \\
\text { Cole, D. A., } \\
\text { \& Martin, N. } \\
\text { C. }(2013) .\end{array}$ & $\begin{array}{l}\text { Flood } \\
\text { (USA) }\end{array}$ & $\begin{array}{l}\text { Pre-post } \\
\text { (without } \\
\text { control } \\
\text { group) }\end{array}$ & Individual & $\begin{array}{l}239 \text { students } \\
\text { aged 10-15 } \\
\text { years }\end{array}$ & $\begin{array}{l}\text { preflood } \\
\text { rumination, } \\
\text { depressive } \\
\text { symptoms, } \\
\text { flood-related } \\
\text { experiences }\end{array}$ & $\begin{array}{l}\text { Preflood } \\
\text { rumination } \\
\text { Preflood } \\
\text { depression }\end{array}$ & Age & $\begin{array}{l}6 \text { months } \\
\text { pre-disaster } \\
\text { (T1) } \\
10 \text { days post } \\
\text { disaster } \\
\text { (T2) }\end{array}$ & $\begin{array}{l}\text { Depressive } \\
\text { Reactions } \\
\text { Postflood } \\
\text { rumination } \\
\text { Postflood } \\
\text { depression }\end{array}$ \\
\hline $\begin{array}{l}\text { Feo, P., Di } \\
\text { Gioia, S., } \\
\text { Carloni, E., } \\
\text { Vitiello, B., } \\
\text { Tozzi, A. E., } \\
\text { \& Vicari, S. } \\
\text { (2014). }\end{array}$ & $\begin{array}{l}\text { Earthquake } \\
\text { (Italy) }\end{array}$ & $\begin{array}{l}\text { Cross- } \\
\text { sectional }\end{array}$ & $\begin{array}{l}\text { Individual } \\
\text { and } \\
\text { Interpersona } \\
1\end{array}$ & $\begin{array}{l}1839 \\
\text { children } \\
\text { aged 3-14 } \\
\text { years }\end{array}$ & $\begin{array}{l}\text { socio- } \\
\text { demographic } \\
\text {, health, } \\
\text { family data, } \\
\text { proximity to } \\
\text { the } \\
\text { epicentre, } \\
\text { damages to } \\
\text { the house, } \\
\text { internally } \\
\text { displaced } \\
\text { condition. } \\
\text { parent's loss } \\
\text { of job, } \\
\text { gender, age }\end{array}$ & & & $\begin{array}{l}12 \text { to } 17 \\
\text { months } \\
\text { post disaster }\end{array}$ & $\begin{array}{l}\text { Psychiatric } \\
\text { symptoms }\end{array}$ \\
\hline $\begin{array}{l}\text { Fivush, R., } \\
\text { McDermott } \\
\text { Sales, J., } \\
\text { Goldberg, A., } \\
\text { Bahrick, L., \& } \\
\text { Parker, J. } \\
\text { (2004). }\end{array}$ & $\begin{array}{l}\text { Hurricane } \\
\text { (USA) }\end{array}$ & $\begin{array}{l}\text { Longitudin } \\
\text { al }\end{array}$ & Individual & $\begin{array}{l}100 \text { children } \\
\text { aged } 3-4 \\
\text { years }\end{array}$ & $\begin{array}{l}\text { Time, stress } \\
\text { group, } \\
\text { phase of } \\
\text { hurricane }\end{array}$ & & & $\begin{array}{l}\text { Few months } \\
\text { post disaster } \\
\text { (T1) } \\
6 \text { years post } \\
\text { disaster } \\
(\mathrm{T} 2)\end{array}$ & $\begin{array}{l}\text { Recall of } \\
\text { Hurricane }\end{array}$ \\
\hline $\begin{array}{l}\text { Foa, E. B., } \\
\text { Johnson, K. } \\
\text { M., Feeny, N. } \\
\text { C., \& } \\
\text { Treadwell, K. } \\
\text { R. (2001). }\end{array}$ & $\begin{array}{l}\text { Earthquake } \\
\text { (USA) }\end{array}$ & $\begin{array}{l}\text { Cross- } \\
\text { sectional }\end{array}$ & Individual & $\begin{array}{l}75 \text { children } \\
\text { aged 8-15 } \\
\text { years }\end{array}$ & $\begin{array}{l}\text { Age, } \\
\text { gender }\end{array}$ & & & $\begin{array}{l}2 \text { years } \\
\text { post disaster }\end{array}$ & $\begin{array}{l}\text { PTSD } \\
\text { Depression } \\
\text { Anxiety }\end{array}$ \\
\hline
\end{tabular}




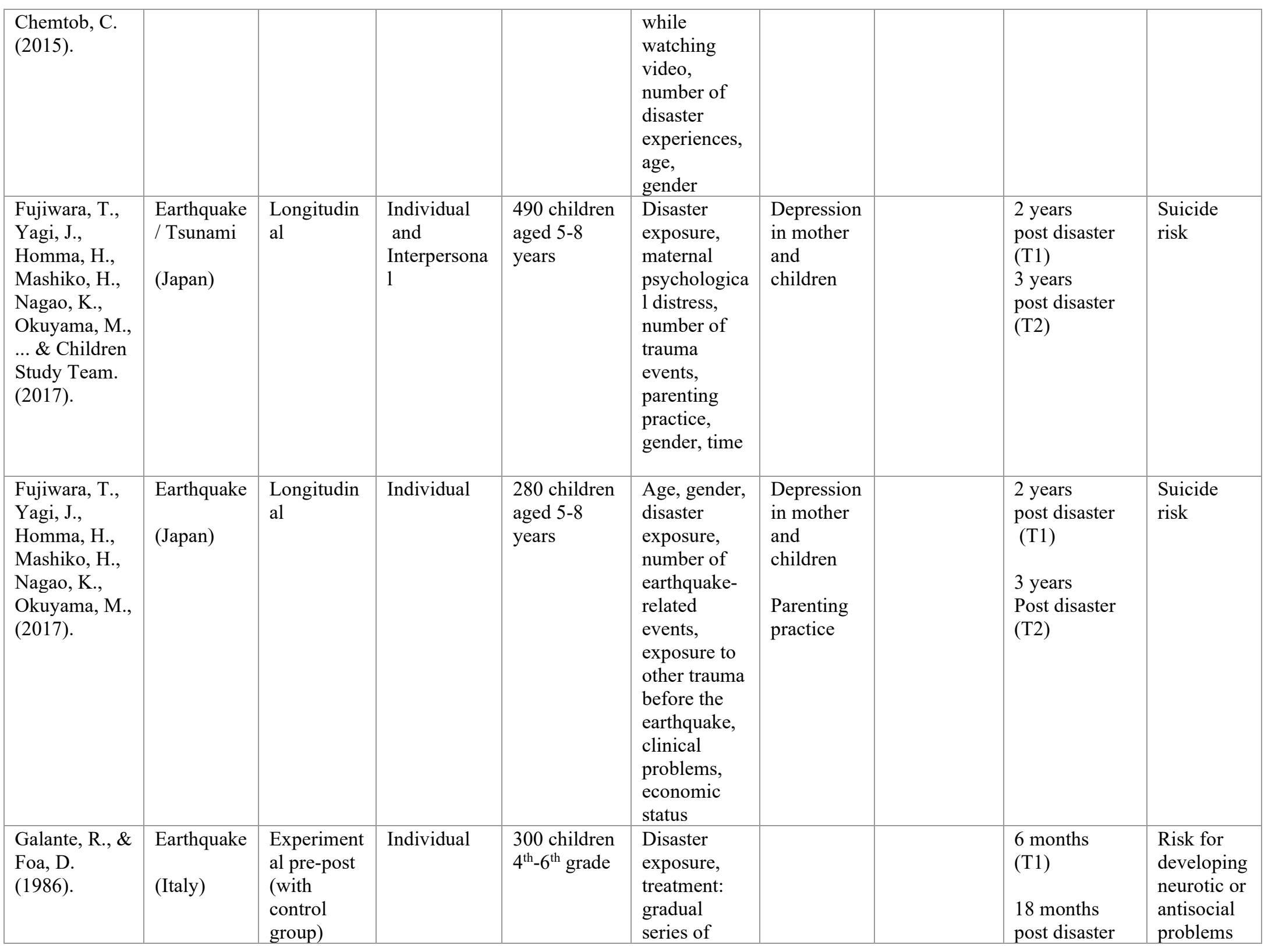




\begin{tabular}{|c|c|c|c|c|c|c|c|}
\hline & & & & & $\begin{array}{l}\text { steps that led } \\
\text { to a } \\
\text { replaying of } \\
\text { the } \\
\text { earthquake }\end{array}$ & $\begin{array}{l}\text { (1 after } \\
\text { pretesting) }\end{array}$ & \\
\hline $\begin{array}{l}\text { Garrison, C. } \\
\text { Z., Bryant, E. } \\
\text { S., Addy, C. } \\
\text { L., Spurrier, } \\
\text { P. G., Freedy, } \\
\text { J. R., \& } \\
\text { Kilpatrick, D. } \\
\text { G. (1995). }\end{array}$ & $\begin{array}{l}\text { Hurricane } \\
\text { (Florida) }\end{array}$ & $\begin{array}{l}\text { Cross- } \\
\text { sectional }\end{array}$ & $\begin{array}{l}\text { Individual, } \\
\text { Interpersona } \\
1 \\
\text { and } \\
\text { Intergroup }\end{array}$ & $\begin{array}{l}370 \text { parent- } \\
\text { adolescent } \\
\text { pairs: } \\
158 \\
\text { hispanics, } \\
116 \text { black } \\
104 \text { white } \\
\\
\text { aged } 12-17 \\
\text { years }\end{array}$ & $\begin{array}{l}\text { Disaster } \\
\text { exposure, } \\
\text { gender, } \\
\text { ethnicity, } \\
\text { household } \\
\text { income, } \\
\text { lifetime } \\
\text { exposure to } \\
\text { undesirable } \\
\text { events } \\
\text { parent fear, } \\
\text { parent PTSD } \\
\text { symptoms }\end{array}$ & $\begin{array}{l}6 \text { months post } \\
\text { hurricane }\end{array}$ & PTSD \\
\hline $\begin{array}{l}\text { Ge, F., Yuan, } \\
\text { M., Li, Y., } \\
\text { Zhang, J., \& } \\
\text { Zhang, W. } \\
\text { (2019) }\end{array}$ & $\begin{array}{l}\text { Earthquake } \\
\text { (China) }\end{array}$ & $\begin{array}{l}\text { Longitudin } \\
\text { al }\end{array}$ & Individual & $\begin{array}{l}1623 \\
\text { primary and } \\
\text { secondary } \\
\text { school } \\
\text { children }\end{array}$ & $\begin{array}{l}\text { socio- } \\
\text { demographic } \\
\text { characteristi } \\
\text { cs } \\
\text { PTSD }\end{array}$ & $\begin{array}{l}2 \text { weeks, } 3 \\
\text { months and } 6 \\
\text { months } \\
\text { after the } \\
\text { earthquake }\end{array}$ & PTSD \\
\hline $\begin{array}{l}\text { Giannopoulou } \\
\text {, I., Smith, P., } \\
\text { Ecker, C., } \\
\text { Strouthos, M., } \\
\text { Dikaiakou, } \\
\text { A., \& Yule, } \\
\text { W. (2006). }\end{array}$ & $\begin{array}{l}\text { Earthquake } \\
\text { (Greece) }\end{array}$ & $\begin{array}{l}\text { Cross- } \\
\text { sectional }\end{array}$ & Individual & $\begin{array}{l}2037 \\
\text { children and } \\
\text { adolescents, } \\
\text { aged 9-17 } \\
\text { years }\end{array}$ & Age, gender & $\begin{array}{l}6-7 \text { months } \\
\text { post disaster }\end{array}$ & $\begin{array}{l}\text { Post- } \\
\text { traumatic } \\
\text { stress } \\
\text { reactions. } \\
\text { PTSD }\end{array}$ \\
\hline $\begin{array}{l}\text { Giannopoulou } \\
\text {, I., Strouthos, } \\
\text { M., Smith, P., } \\
\text { Dikaiakou, } \\
\text { A., } \\
\text { Galanopoulou }\end{array}$ & $\begin{array}{l}\text { Earthquake } \\
\text { (Greece) }\end{array}$ & $\begin{array}{l}\text { Cross- } \\
\text { sectional }\end{array}$ & Individual & $\begin{array}{l}2037 \text { childre } \\
\mathrm{n} \text { and } \\
\text { adolescents } \\
\text { aged } 9-17 \\
\text { years }\end{array}$ & $\begin{array}{l}\text { Different } \\
\text { types of } \\
\text { exposure, } \\
\text { objective } \\
\text { and } \\
\text { subjective }\end{array}$ & $\begin{array}{l}\text { 6-7 months } \\
\text { post disaster }\end{array}$ & $\begin{array}{l}\text { PTSD, } \\
\text { anxiety } \\
\text { and } \\
\text { depression } \\
\text { symptoms }\end{array}$ \\
\hline
\end{tabular}




\section{RESILIENCE IN CHILDREN AFTER NATURAL DISASTERS}

\begin{tabular}{|c|c|c|c|c|c|c|c|}
\hline $\begin{array}{l}\text { V., \& Yule, } \\
\text { W. (2006). }\end{array}$ & & & & & $\begin{array}{l}\text { aspects of } \\
\text { exposure to } \\
\text { the } \\
\text { earthquake, } \\
\text { age, } \\
\text { gender }\end{array}$ & & \\
\hline $\begin{array}{l}\text { Gil-Rivas, V., } \\
\& \text { Kilmer, R. } \\
\text { P. (2013). }\end{array}$ & $\begin{array}{l}\text { Hurricane } \\
\text { (USA) }\end{array}$ & $\begin{array}{l}\text { Longitudin } \\
\text { al }\end{array}$ & $\begin{array}{l}\text { Individual } \\
\text { and } \\
\text { Interpersona } \\
1\end{array}$ & $\begin{array}{l}68 \\
\text { caregiver- } \\
\text { child dyads } \\
\text { age } \mathrm{M}=8.5 \\
\text { years }\end{array}$ & $\begin{array}{l}\text { Caregiver } \\
\text { PTSS and } \\
\text { depressive } \\
\text { symptoms, } \\
\text { coping } \\
\text { advice, } \\
\text { child } \\
\text { perceptions } \\
\text { of caregiver } \\
\text { distress, } \\
\text { unavailabilit } \\
\text { y, } \\
\text { warmth, } \\
\text { caregiver- } \\
\text { child } \\
\text { conflict }\end{array}$ & $\begin{array}{l}12 \text { months } \\
\text { post disaster } \\
\text { (T1) } \\
6-10 \text { months } \\
\text { later } \\
\text { (T2) }\end{array}$ & $\begin{array}{l}\text { PTSS } \\
\text { depressive } \\
\text { symptoms }\end{array}$ \\
\hline $\begin{array}{l}\text { Goenjian, A. } \\
\text { K., Pynoos, R. } \\
\text { S., Steinberg, } \\
\text { A. M., } \\
\text { Najarian, L. } \\
\text { M., Asarnow, } \\
\text { J. R., } \\
\text { Karayan, I., ... } \\
\text { \& Fairbanks, } \\
\text { L. A. (1995). }\end{array}$ & $\begin{array}{l}\text { Earthquake } \\
\text { (Armenia) }\end{array}$ & $\begin{array}{l}\text { Cross- } \\
\text { sectional }\end{array}$ & Individual & $\begin{array}{l}218 \text { children } \\
\text { Spirak } \\
(\mathrm{n}=63, \\
\mathrm{M}=13.7) \\
\text { Gumri } \\
(\mathrm{n}=94, \\
\mathrm{M}=13,1) \\
\text { Yerevan } \\
(\mathrm{n}=61, \\
\mathrm{M}=12.1)\end{array}$ & $\begin{array}{l}\text { Age, gender, } \\
\text { disaster } \\
\text { exposure, } \\
\text { extent of } \\
\text { loss of } \\
\text { relatives and } \\
\text { destruction } \\
\text { of residence }\end{array}$ & $\begin{array}{l}18 \text { months } \\
\text { post disaster }\end{array}$ & $\begin{array}{l}\text { PTSD } \\
\text { depression } \\
\text { separation } \\
\text { anxiety } \\
\text { disorder }\end{array}$ \\
\hline $\begin{array}{l}\text { Goenjian, A. } \\
\text { K., Walling, } \\
\text { D., Steinberg, } \\
\text { A. M., } \\
\text { Roussos, A., }\end{array}$ & $\begin{array}{l}\text { Earthquake } \\
\text { (Armenia) }\end{array}$ & $\begin{array}{l}\text { Cross- } \\
\text { sectional }\end{array}$ & Individual & $\begin{array}{l}48 \text { parentally } \\
\text { bereaved } \\
\text { adolescents } \\
\text { and }\end{array}$ & $\begin{array}{l}\text { Parentally } \\
\text { bereaved } \\
\text { and a } \\
\text { comparison } \\
\text { group after a }\end{array}$ & $\begin{array}{l}\text { Six and an } \\
\text { half year post } \\
\text { disaster }\end{array}$ & $\begin{array}{l}\text { Depression } \\
\text { and PTSD } \\
\text { symptoms }\end{array}$ \\
\hline
\end{tabular}




\begin{tabular}{|c|c|c|c|c|c|c|c|}
\hline $\begin{array}{l}\text { Goenjian, H. } \\
\text { A., \& Pynoos, } \\
\text { R. S. (2009). }\end{array}$ & & & & $\begin{array}{l}44 \text { subjects } \\
\text { with no } \\
\text { parental loss, } \\
\text { aged 11-16 } \\
\text { years }\end{array}$ & $\begin{array}{l}\text { catastrophic } \\
\text { natural } \\
\text { disaster, } \\
\text { loss of both } \\
\text { parents, } \\
\text { loss of } \\
\text { father, } \\
\text { loss of } \\
\text { mother, } \\
\text { no parental } \\
\text { loss }\end{array}$ & & \\
\hline $\begin{array}{l}\text { Gökçen, C., } \\
\text { Şahingöz, M., } \\
\text { \& Annagür, } \\
\text { B. B. (2013). }\end{array}$ & $\begin{array}{l}\text { Earthquake } \\
\text { (Turkey) }\end{array}$ & $\begin{array}{l}\text { Cross- } \\
\text { sectional }\end{array}$ & $\begin{array}{l}\text { Individual } \\
\text { and } \\
\text { Interpersona } \\
1\end{array}$ & $\begin{array}{l}450 \text { children } \\
\text { aged } 12-14 \\
\text { years }\end{array}$ & $\begin{array}{l}\text { Gender, } \\
\text { family type } \\
\text { (traditional } \\
\text { or core), } \\
\text { previous } \\
\text { traumatic } \\
\text { experience } \\
\text { personal, } \\
\text { family } \\
\text { history of } \\
\text { psychiatric } \\
\text { disorders }\end{array}$ & $\begin{array}{l}6 \text { months } \\
\text { post disaster }\end{array}$ & PTSD \\
\hline $\begin{array}{l}\text { Gomez, C. J., } \\
\text { \& Yoshikawa, } \\
\text { H. (2017). }\end{array}$ & $\begin{array}{l}\text { Earthquake } \\
\text { (Chile) }\end{array}$ & $\begin{array}{l}\text { Experiment } \\
\text { al (with } \\
\text { control } \\
\text { group) }\end{array}$ & $\begin{array}{l}\text { Individual } \\
\text { and } \\
\text { Interpersona } \\
1\end{array}$ & $\begin{array}{l}698 \text { children } \\
\text { exposed } \\
\text { and } \\
720 \text { children } \\
\text { no-exposed } \\
\text { aged } 53 \\
\text { months }\end{array}$ & $\begin{array}{l}\text { Disaster } \\
\text { exposure, } \\
\text { earthquake } \\
\text { related } \\
\text { stressors, } \\
\text { gender, age, } \\
\text { parent's } \\
\text { education, } \\
\text { child prior } \\
\text { experience } \\
\text { in an early } \\
\text { education } \\
\text { setting, } \\
\text { socio- } \\
\text { economic } \\
\text { status, }\end{array}$ & $\begin{array}{l}\text { 3-12 weeks } \\
\text { post disaster }\end{array}$ & $\begin{array}{l}\text { Cognitive } \\
\text { function } \\
\text { skills } \\
\text { Early } \\
\text { language, } \\
\text { pre- } \\
\text { literacy, } \\
\text { mathemati } \\
\text { cs and } \\
\text { executive } \\
\text { function } \\
\text { outcomes }\end{array}$ \\
\hline
\end{tabular}




\section{RESILIENCE IN CHILDREN AFTER NATURAL DISASTERS}

\begin{tabular}{|c|c|c|c|c|c|c|c|}
\hline & & & & & $\begin{array}{l}\text { classroom- } \\
\text { level } \\
\text { teacher's, } \\
\text { parent's } \\
\text { earthquake } \\
\text { related- } \\
\text { stressors }\end{array}$ & & \\
\hline $\begin{array}{l}\text { Green, B. L., } \\
\text { Korol, M., } \\
\text { Grace, M. C., } \\
\text { Vary, M. G., } \\
\text { Leonard, A. } \\
\text { C., Gleser, G. } \\
\text { C., \& } \\
\text { Smitson- } \\
\text { Cohen, S. } \\
\text { (1991). }\end{array}$ & $\begin{array}{l}\text { Flood } \\
\text { (USA) }\end{array}$ & $\begin{array}{l}\text { Cross- } \\
\text { sectional }\end{array}$ & $\begin{array}{l}\text { Individual } \\
\text { and } \\
\text { Interpersona } \\
1\end{array}$ & $\begin{array}{l}179 \text { children } \\
\text { aged } 2-15 \\
\text { years }\end{array}$ & $\begin{array}{l}\text { Age, gender, } \\
\text { disaster } \\
\text { exposure, } \\
\text { parental } \\
\text { functioning, } \\
\text { family } \\
\text { atmosphere }\end{array}$ & $\begin{array}{l}2 \text { years } \\
\text { post disaster }\end{array}$ & PTSD \\
\hline $\begin{array}{l}\text { Groome, D., } \\
\& \text { Soureti, A. } \\
(2004) .\end{array}$ & $\begin{array}{l}\text { Earthquake } \\
\text { (Greece) }\end{array}$ & $\begin{array}{l}\text { Cross- } \\
\text { sectional }\end{array}$ & Individual & $\begin{array}{l}178 \text { children } \\
\text { aged } 9-14 \\
\text { years }\end{array}$ & $\begin{array}{l}\text { Pproximity } \\
\text { to the } \\
\text { epicentre, } \\
\text { exposure to } \\
\text { threat, } \\
\text { gender, } \\
\text { age }\end{array}$ & $\begin{array}{l}5 \text { months } \\
\text { post disaster }\end{array}$ & $\begin{array}{l}\text { PTSD } \\
\text { Anxiety }\end{array}$ \\
\hline $\begin{array}{l}\text { Hafstad, G. } \\
\text { S., Gil-Rivas, } \\
\text { V., Kilmer, R. } \\
\text { P., \& Raeder, } \\
\text { S. (2010). }\end{array}$ & $\begin{array}{l}\text { Tsunami } \\
\text { (Thailand) }\end{array}$ & $\begin{array}{l}\text { Longitudin } \\
\text { al }\end{array}$ & $\begin{array}{l}\text { Individual } \\
\text { and } \\
\text { Interpersona } \\
1\end{array}$ & $\begin{array}{l}105 \\
\text { morwegian } \\
\text { children } \\
\text { aged 6-17 } \\
\text { years } \\
\text { and their } \\
\text { parents } \\
(\mathrm{N}=67)\end{array}$ & $\begin{array}{l}\text { Subjective } \\
\text { exposure } \\
\text { and } \\
\text { concurrent } \\
\text { PTSS, } \\
\text { objective } \\
\text { exposure, } \\
\text { parent ptg }\end{array}$ & $\begin{array}{l}6 \text { months post } \\
\text { disaster } \\
\text { (T1), } \\
10 \text { months } \\
\text { (T2), } \\
2,5 \text { years } \\
\text { post disaster } \\
\text { (T3) }\end{array}$ & $\begin{array}{l}\text { Posttrauma } \\
\text { tic Growth }\end{array}$ \\
\hline $\begin{array}{l}\text { Hafstad, G. } \\
\text { S., Kilmer, R. } \\
\text { P., \& Gil- } \\
\text { Rivas, V. } \\
(2011) .\end{array}$ & $\begin{array}{l}\text { Tsunami } \\
\text { (Southeast } \\
\text { Asia) }\end{array}$ & $\begin{array}{l}\text { Longitudin } \\
\text { al }\end{array}$ & Individual & $\begin{array}{l}105 \\
\text { norwegian } \\
\text { children } \\
\text { aged 6-17 } \\
\text { years }\end{array}$ & $\begin{array}{l}\text { Age gender } \\
\text { prior } \\
\text { adversity } \\
\text { exposure } \\
\text { objective } \\
\text { tsunami- }\end{array}$ & $\begin{array}{l}10 \text { and } 30 \\
\text { months } \\
\text { post disaster }\end{array}$ & $\begin{array}{l}\text { Posttrauma } \\
\text { tic Growth }\end{array}$ \\
\hline
\end{tabular}




\section{RESILIENCE IN CHILDREN AFTER NATURAL DISASTERS}

\begin{tabular}{|c|c|c|c|c|c|c|c|}
\hline & & & & & $\begin{array}{l}\text { related } \\
\text { exposure } \\
\text { subjective } \\
\text { tsunami- } \\
\text { related } \\
\text { reactions, } \\
\text { tsunami- } \\
\text { related } \\
\text { traumatic } \\
\text { events, } \\
\text { post } \\
\text { traumatic } \\
\text { symptoms }\end{array}$ & & \\
\hline $\begin{array}{l}\text { Hall, A., \& } \\
\text { Kirby, H. } \\
(2010) .\end{array}$ & $\begin{array}{l}\text { Earthquake } \\
\text { (Pakistan) }\end{array}$ & $\begin{array}{l}\text { Cross- } \\
\text { sectional }\end{array}$ & $\begin{array}{l}\text { Individual } \\
\text { and } \\
\text { Interpersona } \\
1\end{array}$ & $\begin{array}{l}2032 \\
\text { children } \\
5-14 \text { years }\end{array}$ & $\begin{array}{l}\text { Age, gender, } \\
\text { enrolment } \\
\text { status, } \\
\text { socio- } \\
\text { economic } \\
\text { characteristi } \\
\text { cs }\end{array}$ & $\begin{array}{l}12 \text { months } \\
\text { post disaster }\end{array}$ & $\begin{array}{l}\text { Health and } \\
\text { nutritional } \\
\text { status }\end{array}$ \\
\hline $\begin{array}{l}\text { Hambrick, E. } \\
\text { P., O'connor, } \\
\text { B. M., \& } \\
\text { Vernberg, E. } \\
\text { M. (2016). }\end{array}$ & $\begin{array}{l}\text { Tornado } \\
\text { (USA) }\end{array}$ & $\begin{array}{l}\text { Longitudin } \\
\text { al }\end{array}$ & Individual & $\begin{array}{l}50 \text { children } \\
\text { aged } 8-12 \\
\text { years }\end{array}$ & $\begin{array}{l}\text { Disaster } \\
\text { exposure, } \\
\text { life- } \\
\text { threatening } \\
\text { experiences, } \\
\text { disruptive } \\
\text { life } \\
\text { experiences } \\
\text { during and } \\
\text { after the } \\
\text { tornado, } \\
\text { PTSS } \\
\text { severity, } \\
\text { time }\end{array}$ & $\begin{array}{l}12 \text { months } \\
\text { post disaster } \\
\text { (T1) } \\
3 \text { montsh after } \\
\text { (T2) }\end{array}$ & $\begin{array}{l}\text { Happiness, } \\
\text { upset and } \\
\text { nervousnes } \\
\mathrm{s} \\
\text { Perception } \\
\text { s of } \\
\text { research }\end{array}$ \\
\hline $\begin{array}{l}\text { Hambrick, E. } \\
\text { P., Vernberg, } \\
\text { E. M., } \\
\text { Greenhoot, A. } \\
\text { F., \& }\end{array}$ & $\begin{array}{l}\text { Tornado } \\
\text { (USA) }\end{array}$ & $\begin{array}{l}\text { Cross- } \\
\text { sectional }\end{array}$ & Individual & $\begin{array}{l}50 \text { children } \\
8-12 \text { year- } \\
\text { olds } \\
\text { and their } \\
\text { mothers }\end{array}$ & $\begin{array}{l}\text { Children's } \\
\text { processing } \\
\text { in trauma } \\
\text { recollections }\end{array}$ & $\begin{array}{l}1 \text { week after } \\
\text { tornado }\end{array}$ & $\begin{array}{l}\text { PTSD, } \\
\text { depression, } \\
\text { and } \\
\text { anxiety }\end{array}$ \\
\hline
\end{tabular}




\begin{tabular}{|c|c|c|c|c|c|c|c|c|}
\hline $\begin{array}{l}\text { Hendrickson, } \\
\text { M. L. (2017) }\end{array}$ & & & & & $\begin{array}{l}\text { level of } \\
\text { exposure, } \\
\text { Family } \\
\text { income, } \\
\text { Age } \\
\text { Gender } \\
\text { Verbal } \\
\text { ability }\end{array}$ & & & \\
\hline $\begin{array}{l}\text { Han, L., } \\
\text { Zhang, Y., \& } \\
\text { Zheng, Y. } \\
\text { (2012). }\end{array}$ & $\begin{array}{l}\text { Earthquake } \\
\text { (China) }\end{array}$ & $\begin{array}{l}\text { Longitudin } \\
\text { al }\end{array}$ & Individual & $\begin{array}{l}188 \text { children } \\
\text { aged } 11-15 \\
\text { years }\end{array}$ & $\begin{array}{l}\text { Time, } \\
\text { gender }\end{array}$ & & $\begin{array}{l}2 \text { weeks and } \\
12 \text { months } \\
\text { post disaster }\end{array}$ & $\begin{array}{l}\text { Mental } \\
\text { health and } \\
\text { coping } \\
\text { styles }\end{array}$ \\
\hline $\begin{array}{l}\text { Hansel, T. C., } \\
\text { Osofsky, J. } \\
\text { D., Osofsky, } \\
\text { H. J., \& } \\
\text { Friedrich, P. } \\
(2013) .\end{array}$ & $\begin{array}{r}\text { Hurricane } \\
\text { (USA) }\end{array}$ & $\begin{array}{l}\text { Cross- } \\
\text { sectional }\end{array}$ & Individual & $\begin{array}{l}795 \text { children } \\
\text { and } \\
\text { adolescents } \\
\left(5^{\text {th }}-12^{\text {th }}\right. \\
\text { grades })\end{array}$ & $\begin{array}{l}\text { Age (3 } \\
\text { groups), } \\
\text { relocation } \\
\text { groupings, } \\
\text { disaster } \\
\text { exposure }\end{array}$ & & $\begin{array}{l}4 \text { years } \\
\text { post disaster }\end{array}$ & $\begin{array}{l}\text { Longer- } \\
\text { term } \\
\text { psychologi } \\
\text { cal } \\
\text { symptoms: } \\
\text { trauma, } \\
\text { depression, } \\
\text { and } \\
\text { posttrauma } \\
\text { tic stress }\end{array}$ \\
\hline $\begin{array}{l}\text { Hausman, E. } \\
\text { M., Black, S. } \\
\text { R., Bromet, } \\
\text { E., Carlson, } \\
\text { G., Danzig, } \\
\text { A., Kotov, R., } \\
\text { \& Klein, D. } \\
\text { N. (2020) }\end{array}$ & $\begin{array}{l}\text { Hurricane } \\
\text { (U.S.A.) }\end{array}$ & $\begin{array}{l}\text { Longitudin } \\
\text { al }\end{array}$ & $\begin{array}{l}\text { Individual } \\
\text { Intepersonal }\end{array}$ & $\begin{array}{l}347 \text { pairs } \\
\text { mother-child } \\
9 \text { years old }\end{array}$ & $\begin{array}{l}\text { Family's } \\
\text { stress } \\
\text { exposure } \\
\text { Predisaster } \\
\text { symptom }\end{array}$ & & $\begin{array}{l}1 \text { year before } \\
\text { hurricane,. } 8 \\
\text { weeks after } \\
\text { the hurricane }\end{array}$ & $\begin{array}{l}\text { depression } \\
\text { anxiety }\end{array}$ \\
\hline $\begin{array}{l}\text { Hensley- } \\
\text { Maloney, L., } \\
\text { \& Varela, R. } \\
\text { E. (2009). }\end{array}$ & $\begin{array}{l}\text { Hurricane } \\
\text { (USA) }\end{array}$ & $\begin{array}{l}\text { Cross- } \\
\text { sectional }\end{array}$ & Individual & $\begin{array}{l}302 \text { children } \\
6 \text { th and } 7 \text { th } \\
\text { graders }(\mathrm{T} 1) \\
110 \text { children } \\
6 \text { th and } 7 \text { th } \\
\text { graders (T2) }\end{array}$ & $\begin{array}{l}\text { Anxiety } \\
\text { sensitivity, } \\
\text { gender }\end{array}$ & $\begin{array}{l}\text { Trauma } \\
\text { exposure }\end{array}$ & $\begin{array}{l}5-8 \text { months } \\
\text { post disaster } \\
\text { (T1) } \\
\text { 17-18 months } \\
\text { post disaster } \\
\text { (T2) }\end{array}$ & $\begin{array}{l}\text { Panic } \\
\text { symptoms }\end{array}$ \\
\hline
\end{tabular}


RESILIENCE IN CHILDREN AFTER NATURAL DISASTERS

\begin{tabular}{|c|c|c|c|c|c|c|c|}
\hline $\begin{array}{l}\text { Hizli, F. G., } \\
\text { Taskintuna, } \\
\text { N., Isikli, S., } \\
\text { Kilic, C., \& } \\
\text { Zileli, L. } \\
\text { (2009). }\end{array}$ & $\begin{array}{l}\text { Earthquake } \\
\text { (Turkey) }\end{array}$ & $\begin{array}{l}\text { Cross- } \\
\text { sectional }\end{array}$ & $\begin{array}{l}\text { Individual } \\
\text { and } \\
\text { Interpersona } \\
1\end{array}$ & $\begin{array}{l}1999 \\
\text { children and } \\
\text { adolescents, } \\
\text { aged 8-18 } \\
\text { years }\end{array}$ & $\begin{array}{l}\text { Socio- } \\
\text { demographic } \\
\text { characteristi } \\
\text { cs, } \\
\text { loss of } \\
\text { source, and } \\
\text { loss of social } \\
\text { support, } \\
\text { fear and } \\
\text { avoidance, } \\
\text { post- } \\
\text { earthquake } \\
\text { life events, } \\
\text { impact of } \\
\text { earthquake }\end{array}$ & $\begin{array}{l}4 \text { years } \\
\text { post disaster }\end{array}$ & $\begin{array}{l}\text { PTSD } \\
\text { Depression }\end{array}$ \\
\hline $\begin{array}{l}\text { Hlodversdottir } \\
\text {, H., } \\
\text { Thorsteinsdott } \\
\text { ir, H., } \\
\text { Thordardottir, } \\
\text { E. B., } \\
\text { Njardvik, U., } \\
\text { Petursdottir, } \\
\text { G., \& } \\
\text { Hauksdottir, } \\
\text { A. (2018) }\end{array}$ & $\begin{array}{l}\text { Volcanic } \\
\text { eruption } \\
\text { (Iceland) }\end{array}$ & $\begin{array}{l}\text { Cross- } \\
\text { sectional }\end{array}$ & $\begin{array}{l}\text { Individual } \\
\text { Interpersona } \\
1\end{array}$ & $\begin{array}{l}1615 \text { adult } \\
\text { and their } \\
\text { children }\end{array}$ & $\begin{array}{l}\text { exposure } \\
\text { level } \\
\text { gender } \\
\text { age } \\
\text { education } \\
\text { level } \\
\text { marital } \\
\text { status } \\
\text { house } \\
\text { damage } \\
\text { Parental } \\
\text { mental } \\
\text { health }\end{array}$ & $\begin{array}{l}6-9 \text { months } \\
\text { after the } \\
\text { eruption } \\
3 \text { years after } \\
\text { eruption }\end{array}$ & $\begin{array}{l}\text { physical } \\
\text { and } \\
\text { mental } \\
\text { health } \\
\text { symptoms }\end{array}$ \\
\hline $\begin{array}{l}\text { Houlihan, D., } \\
\text { Ries, B. J., } \\
\text { Polusny, M. } \\
\text { A., \& Hanson, } \\
\text { C. N. (2008). }\end{array}$ & $\begin{array}{l}\text { Tornado } \\
\text { (USA) }\end{array}$ & $\begin{array}{l}\text { Cross- } \\
\text { sectional }\end{array}$ & Individual & $\begin{array}{l}95 \text { students } \\
3^{\text {rd }}-12^{\text {th }} \\
\text { grade }\end{array}$ & $\begin{array}{l}\text { Disaster } \\
\text { exposure, } \\
\text { home } \\
\text { relocation, } \\
\text { PTSD } \\
\text { symptoms }\end{array}$ & $\begin{array}{l}\text { 5-6 weeks } \\
\text { post disaster }\end{array}$ & $\begin{array}{l}\text { Level of } \\
\text { life } \\
\text { satisfaction } \\
\text { Total } \\
\text { behavior } \\
\text { problems }\end{array}$ \\
\hline
\end{tabular}




\begin{tabular}{|c|c|c|c|c|c|c|c|}
\hline & & & & & & & $\begin{array}{l}\text { Internalizi } \\
\text { ng } \\
\text { behaviors, } \\
\text { and } \\
\text { externalizi } \\
\text { ng } \\
\text { behaviors }\end{array}$ \\
\hline $\begin{array}{l}\text { Hsu, C. C., } \\
\text { Chong, M. Y., } \\
\text { Yang, P., \& } \\
\text { Yen, C. F. } \\
(2002) .\end{array}$ & $\begin{array}{l}\text { Earthquake } \\
\text { (Taiwan) }\end{array}$ & $\begin{array}{l}\text { Cross- } \\
\text { sectional }\end{array}$ & Individual & $\begin{array}{l}323 \\
\text { adolescents } \\
\text { aged 12-14 } \\
\text { years }\end{array}$ & $\begin{array}{l}\text { Disaster } \\
\text { exposure, } \\
\text { PTSD }\end{array}$ & $\begin{array}{l}6 \text { weeks } \\
\text { post disaster }\end{array}$ & PTSD \\
\hline $\begin{array}{l}\text { Huzziff, C. } \\
\text { A., \& Ronan, } \\
\text { K. R. (1999). }\end{array}$ & $\begin{array}{l}\text { Eruptions } \\
\text { (New } \\
\text { Zealand) }\end{array}$ & $\begin{array}{l}\text { Longitudin } \\
\text { al }\end{array}$ & $\begin{array}{l}\text { Individual } \\
\text { and } \\
\text { Interpersona } \\
1\end{array}$ & $\begin{array}{l}187 \text { students } \\
\text { age } M=10 \\
\text { years }\end{array}$ & $\begin{array}{l}\text { PTSD. } \\
\text { disaster } \\
\text { exposure, } \\
\text { demographic } \\
\text { s, home } \\
\text { factors, } \\
\text { negative, } \\
\text { cognitive } \\
\text { and } \\
\text { emotional } \\
\text { style } \\
\text { coping, } \\
\text { time }\end{array}$ & $\begin{array}{l}1 \text { month } \\
\text { post-disaster } \\
3 \text { months } \\
\text { post-disaster }\end{array}$ & Coping \\
\hline $\begin{array}{l}\text { Itagaki, S., } \\
\text { Harigane, M., } \\
\text { Maeda, M., } \\
\text { Yasumura, S., } \\
\text { Suzuki, Y., } \\
\text { Mashiko, H., } \\
\text {... \& Mental } \\
\text { Health Group } \\
\text { of the } \\
\text { Fukushima } \\
\text { Health } \\
\text { Management } \\
\text { Survey. } \\
\text { (2017). }\end{array}$ & $\begin{array}{l}\text { Earthquake } \\
\text { (Japan) }\end{array}$ & $\begin{array}{l}\text { Cross- } \\
\text { sectional }\end{array}$ & Individual & $\begin{array}{l}10824 \\
\text { children } \\
\text { aged } 6-15 \\
\text { years }\end{array}$ & $\begin{array}{l}\text { Gender, } \\
\text { age, place } \\
\text { of residence, } \\
\text { exercise } \\
\text { category, } \\
\text { treatment for } \\
\text { illnesses and } \\
\text { experienced } \\
\text { the nuclear } \\
\text { reactor } \\
\text { accident }\end{array}$ & $\begin{array}{l}12 \text { months } \\
\text { post disaster }\end{array}$ & $\begin{array}{l}\text { Mental } \\
\text { health }\end{array}$ \\
\hline
\end{tabular}




\section{RESILIENCE IN CHILDREN AFTER NATURAL DISASTERS}

\begin{tabular}{|c|c|c|c|c|c|c|c|}
\hline $\begin{array}{l}\text { Iwadare, Y., } \\
\text { Usami, M., } \\
\text { Suzuki, Y., } \\
\text { Ushijima, H., } \\
\text { Tanaka, T., } \\
\text { Watanabe, K., } \\
\text {.. \& Saito, K. } \\
\text { (2014). }\end{array}$ & $\begin{array}{l}\text { Earthquake } \\
\text { and } \\
\text { Tsunami } \\
\text { (Japan) }\end{array}$ & $\begin{array}{l}\text { Longitudin } \\
\text { al }\end{array}$ & Individual & $\begin{array}{l}3795 \\
\text { children }\left(5^{\text {th }}-\right. \\
\left.6^{\text {th }} \text { grades }\right) \\
\text { and } \\
\text { adolescents } \\
\left(8^{\text {th }}-9^{\text {th }}\right. \\
\text { grades })\end{array}$ & $\begin{array}{l}\text { Age, gender, } \\
\text { degree of } \\
\text { disaster } \\
\text { experienced }\end{array}$ & $\begin{array}{l}8-20 \text { months } \\
\text { post disaster }\end{array}$ & $\begin{array}{l}\text { Posttrauma } \\
\text { tic } \\
\text { symptoms }\end{array}$ \\
\hline $\begin{array}{l}\text { Jeney- } \\
\text { Gammon, P., } \\
\text { Daugherty, T. } \\
\text { K., Finch Jr, } \\
\text { A. J., Belter, } \\
\text { R. W., \& } \\
\text { Foster, K. Y. } \\
\text { (1993). }\end{array}$ & $\begin{array}{l}\text { Hurricane } \\
\text { (USA) }\end{array}$ & $\begin{array}{l}\text { Cross- } \\
\text { sectional }\end{array}$ & $\begin{array}{l}\text { Individual } \\
\text { and } \\
\text { Interpersona } \\
1\end{array}$ & $\begin{array}{l}257 \text { children } \\
3^{\text {rd }}-5^{\text {th }} \text { grade }\end{array}$ & $\begin{array}{l}\text { Children's } \\
\text { coping } \\
\text { styles, } \\
\text { cognitive } \\
\text { restructuring } \\
\text {, seeking } \\
\text { social } \\
\text { support, } \\
\text { social } \\
\text { withdrawal, } \\
\text { self-blame, } \\
\text { emotional } \\
\text { regulation }\end{array}$ & $\begin{array}{l}5 \text { months } \\
\text { post disaster }\end{array}$ & $\begin{array}{l}\text { Depressive } \\
\text { symptoms }\end{array}$ \\
\hline $\begin{array}{l}\text { Jia, Z., Tian, } \\
\text { W., He, X., } \\
\text { Liu, W., Jin, } \\
\text { C., \& Ding, } \\
\text { H. (2010). }\end{array}$ & $\begin{array}{l}\text { Earthquake } \\
\text { (China) }\end{array}$ & $\begin{array}{l}\text { Cross- } \\
\text { sectional }\end{array}$ & Individual & $\begin{array}{l}596 \text { children } \\
\text { aged } 8-16 \\
\text { years }\end{array}$ & $\begin{array}{l}\text { Disaster } \\
\text { exposure, } \\
\text { utilization of } \\
\text { mental } \\
\text { health } \\
\text { services, } \\
\text { health- } \\
\text { related } \\
\text { quality of } \\
\text { life }\end{array}$ & $\begin{array}{l}15 \text { months } \\
\text { post disaster }\end{array}$ & $\begin{array}{l}\text { Mental } \\
\text { health and } \\
\text { quality of } \\
\text { life }\end{array}$ \\
\hline $\begin{array}{l}\text { Jieling, C., \& } \\
\text { Xinchun, W. } \\
(2017) .\end{array}$ & $\begin{array}{l}\text { Earthquake } \\
\text { (China) }\end{array}$ & $\begin{array}{l}\text { Cross- } \\
\text { sectional }\end{array}$ & $\begin{array}{l}\text { Individual } \\
\text { and } \\
\text { Interpersona } \\
1\end{array}$ & $\begin{array}{l}618 \\
\text { participants } \\
\text { (aged } \\
\mathrm{M}=12.26 \text { ) }\end{array}$ & $\begin{array}{l}\text { Disaster } \\
\text { exposure, } \\
\text { social } \\
\text { support }\end{array}$ & $\begin{array}{l}8 \text { months } \\
\text { post disaster }\end{array}$ & $\begin{array}{l}\text { Post- } \\
\text { traumatic } \\
\text { stress } \\
\text { symptoms. } \\
\text { Post- } \\
\text { traumatic } \\
\text { growth }\end{array}$ \\
\hline
\end{tabular}




\section{RESILIENCE IN CHILDREN AFTER NATURAL DISASTERS}

\begin{tabular}{|c|c|c|c|c|c|c|c|c|}
\hline $\begin{array}{l}\text { Jones, R. T., } \\
\text { Frary, R., } \\
\text { Cunningham, } \\
\text { P., Weddle, J. } \\
\text { D., \& Kaiser, } \\
\text { L. (2001). }\end{array}$ & $\begin{array}{l}\text { Hurricane } \\
\text { (USA) }\end{array}$ & $\begin{array}{l}\text { Cross- } \\
\text { sectional }\end{array}$ & Individual & $\begin{array}{l}142 \\
\text { elementary } \\
\text { school } \\
\text { children } \\
\text { aged } 8-11 \\
\text { years, } \\
69 \\
\text { adolescents } \\
\text { aged 12-15 } \\
\text { years }\end{array}$ & $\begin{array}{l}\text { Gender, } \\
\text { age and race } \\
\text { appraisal } \\
\text { of the } \\
\text { severity of } \\
\text { the event, } \\
\text { life threat, } \\
\text { loss/injury } \\
\text { factor }\end{array}$ & & $\begin{array}{l}6 \text { months } \\
\text { post disaster }\end{array}$ & $\begin{array}{l}\text { Psychologi } \\
\text { cal effects: } \\
\text { intrusion } \\
\text { and } \\
\text { avoidance }\end{array}$ \\
\hline $\begin{array}{l}\text { Jurgens, J. J., } \\
\text { Houlihan, D., } \\
\text { \& Schwartz, } \\
\text { C. (1996). }\end{array}$ & $\begin{array}{l}\text { Tornado } \\
\text { (USA) }\end{array}$ & $\begin{array}{l}\text { Cross - } \\
\text { sectional }\end{array}$ & Individual & $\begin{array}{l}62 \\
\text { adolescents } \\
12-18 \text { years }\end{array}$ & $\begin{array}{l}\text { School } \\
\text { relocation, } \\
\text { disaster } \\
\text { exposure, } \\
\text { age }\end{array}$ & & $\begin{array}{l}3 \text { months } \\
\text { post disaster }\end{array}$ & $\begin{array}{l}\text { PTSD } \\
\begin{array}{l}\text { Self } \\
\text { efficacy }\end{array} \\
\begin{array}{l}\text { Future } \\
\text { orientation }\end{array} \\
\text { Academic } \\
\text { achieveme } \\
\text { nt }\end{array}$ \\
\hline $\begin{array}{l}\text { Juth, V., } \\
\text { Silver, R. C., } \\
\text { Seyle, D. C., } \\
\text { Widyatmoko, } \\
\text { C. S., \& Tan, } \\
\text { E. T. } \\
(2015) .\end{array}$ & $\begin{array}{l}\text { Earthquake } \\
\text { (Indonesia) }\end{array}$ & $\begin{array}{l}\text { Cross- } \\
\text { sectional }\end{array}$ & $\begin{array}{l}\text { Individual } \\
\text { and } \\
\text { Interpersona } \\
1\end{array}$ & $\begin{array}{l}397 \text { parent- } \\
\text { child dyads } \\
\text { aged } 8-17 \\
\text { years }\end{array}$ & $\begin{array}{l}\text { Disaster } \\
\text { exposure, } \\
\text { parent's pts } \\
\text { symptoms, } \\
\text { children's } \\
\text { pts } \\
\text { symptoms }\end{array}$ & $\begin{array}{l}\text { Parent's or } \\
\text { children's } \\
\text { genders }\end{array}$ & $\begin{array}{l}3 \text { years } \\
\text { post disaster }\end{array}$ & $\begin{array}{l}\text { Children } \\
\text { and } \\
\text { Parent's } \\
\text { psychologi } \\
\text { cal } \\
\text { distress }\end{array}$ \\
\hline $\begin{array}{l}\text { Kalantari, M., } \\
\text { \& Vostanis, P. } \\
(2010) .\end{array}$ & $\begin{array}{l}\text { Earthquake } \\
\text { (Iran) }\end{array}$ & $\begin{array}{l}\text { Cross- } \\
\text { sectional }\end{array}$ & $\begin{array}{l}\text { Individual } \\
\text { and } \\
\text { Interpersona } \\
1\end{array}$ & $\begin{array}{l}166 \text { children } \\
\text { aged } 7-13 \\
\text { years }\end{array}$ & $\begin{array}{l}\text { Parental loss } \\
\text { vs intact } \\
\text { families, } \\
\text { parent's } \\
\text { psychopatho } \\
\text { logy, gender, } \\
\text { age, } \\
\text { socioeconom } \\
\text { ic status, }\end{array}$ & & $\begin{array}{l}4 \text { years } \\
\text { post disaster }\end{array}$ & $\begin{array}{l}\text { Behaviour } \\
\text { al and } \\
\text { emotional } \\
\text { problems }\end{array}$ \\
\hline
\end{tabular}




\begin{tabular}{|c|c|c|c|c|c|c|c|c|}
\hline & & & & & $\begin{array}{l}\text { housing } \\
\text { status }\end{array}$ & & & \\
\hline $\begin{array}{l}\text { Kar, N., } \\
\text { Mohapatra, P. } \\
\text { K., Nayak, K. } \\
\text { C., Pattanaik, } \\
\text { P., Swain, S. } \\
\text { P., \& Kar, H. } \\
\text { C. (2007). }\end{array}$ & $\begin{array}{l}\text { Cyclone } \\
\text { (India) }\end{array}$ & $\begin{array}{l}\text { Cross- } \\
\text { sectional }\end{array}$ & $\begin{array}{l}\text { Individual } \\
\text { and } \\
\text { Interpersona } \\
1\end{array}$ & $\begin{array}{l}447 \text { children } \\
\text { aged } 7-17 \\
\text { years }\end{array}$ & $\begin{array}{l}\text { Disaster } \\
\text { exposure, } \\
\text { educational } \\
\text { level, } \\
\text { socioeconom } \\
\text { ic status, } \\
\text { disaster } \\
\text { related } \\
\text { experiences }\end{array}$ & & $\begin{array}{l}12 \text { months } \\
\text { post disaster }\end{array}$ & PTSD \\
\hline $\begin{array}{l}\text { Kelley, M. L., } \\
\text { Self-Brown, } \\
\text { S., Le, B., } \\
\text { Bosson, J. V., } \\
\text { Hernandez, B. } \\
\text { C., \& Gordon, } \\
\text { A. T. (2010). }\end{array}$ & $\begin{array}{l}\text { Hurricane } \\
\text { (USA) }\end{array}$ & $\begin{array}{l}\text { Longitudin } \\
\text { al }\end{array}$ & $\begin{array}{l}\text { Individual } \\
\text { and } \\
\text { Interpersona } \\
1\end{array}$ & $\begin{array}{l}381 \text { children } \\
\text { aged } 8-16 \\
\text { years } \\
\text { and } \\
\text { parent }\end{array}$ & $\begin{array}{l}\text { Disaster } \\
\text { exposure, } \\
\text { levels of } \\
\text { child PTSD } \\
\text { at T1 }\end{array}$ & $\begin{array}{l}\text { parent } \\
\text { distress } \\
\text { and } \\
\text { negative } \\
\text { parenting } \\
\text { practices, }\end{array}$ & $\begin{array}{l}\text { 3-7 months } \\
\text { post disaster } \\
\text { (T1) } \\
4-17 \text { months } \\
\text { (T2) } \\
\text { post-disaster }\end{array}$ & PTSD risk \\
\hline $\begin{array}{l}\text { Kessel, E. M., } \\
\text { Nelson, B. D., } \\
\text { Finsaas, M., } \\
\text { Kujawa, A., } \\
\text { Meyer, A., } \\
\text { Bromet, E., } \\
\text {... Klein, D. } \\
\text { N. (2019) }\end{array}$ & $\begin{array}{l}\text { Hurricane } \\
\text { (USA) }\end{array}$ & $\begin{array}{l}\text { Cross- } \\
\text { sectional }\end{array}$ & $\begin{array}{l}\text { Indivudual } \\
\text { Interpersona } \\
1\end{array}$ & $\begin{array}{l}74 \text { children, } \\
9 \text { years old }\end{array}$ & $\begin{array}{l}\text { parenting } \\
\text { styles and } \\
\text { hurricane- } \\
\text { related stress }\end{array}$ & & $\begin{array}{l}8 \text { months } \\
\text { before } 6 \\
\text { weeks and } 9 \\
\text { months after } \\
\text { Hurricane }\end{array}$ & $\begin{array}{l}\text { neural } \\
\text { reactivity }\end{array}$ \\
\hline $\begin{array}{l}\text { Kessel, E. M., } \\
\text { Nelson, B. D., } \\
\text { Kujawa, A., } \\
\text { Hajcak, G., } \\
\text { Kotov, R., } \\
\text { Bromet, E. J., } \\
\text {.. \& Klein, D. } \\
\text { N. (2018). }\end{array}$ & $\begin{array}{l}\text { Hurricane } \\
\text { (USA) }\end{array}$ & $\begin{array}{l}\text { Pre-post } \\
\text { (without } \\
\text { control } \\
\text { group) }\end{array}$ & Individual & $\begin{array}{l}77 \text { children } \\
\text { aged } 9 \text { years }\end{array}$ & $\begin{array}{l}\text { Disaster } \\
\text { exposure }\end{array}$ & & $\begin{array}{l}8 \text { months } \\
\text { pre disaster } \\
9 \text { months } \\
\text { post disaster }\end{array}$ & $\begin{array}{l}\text { Neural } \\
\text { Reactivity } \\
\text { to negative } \\
\text { stimuli }\end{array}$ \\
\hline $\begin{array}{l}\text { Kiliç, C., } \\
\text { Kiliç, E. Z., \& } \\
\text { Aydin, I. O. } \\
\text { (2011). }\end{array}$ & $\begin{array}{l}\text { Earthquake } \\
\text { (Turkey) }\end{array}$ & $\begin{array}{l}\text { Cross- } \\
\text { sectional }\end{array}$ & $\begin{array}{l}\text { Individual } \\
\text { and } \\
\text { Interpersona } \\
1\end{array}$ & $\begin{array}{l}104 \text { children } \\
\text { aged } 8-15 \\
\text { years and } \\
\text { parents }\end{array}$ & $\begin{array}{l}\text { Parental } \\
\text { psychopatho } \\
\text { logy, } \\
\text { disaster } \\
\text { exposure }\end{array}$ & & $\begin{array}{l}4 \text { years } \\
\text { post disaster }\end{array}$ & $\begin{array}{l}\text { Traumatic } \\
\text { stress and } \\
\text { depression }\end{array}$ \\
\hline
\end{tabular}




\section{RESILIENCE IN CHILDREN AFTER NATURAL DISASTERS}

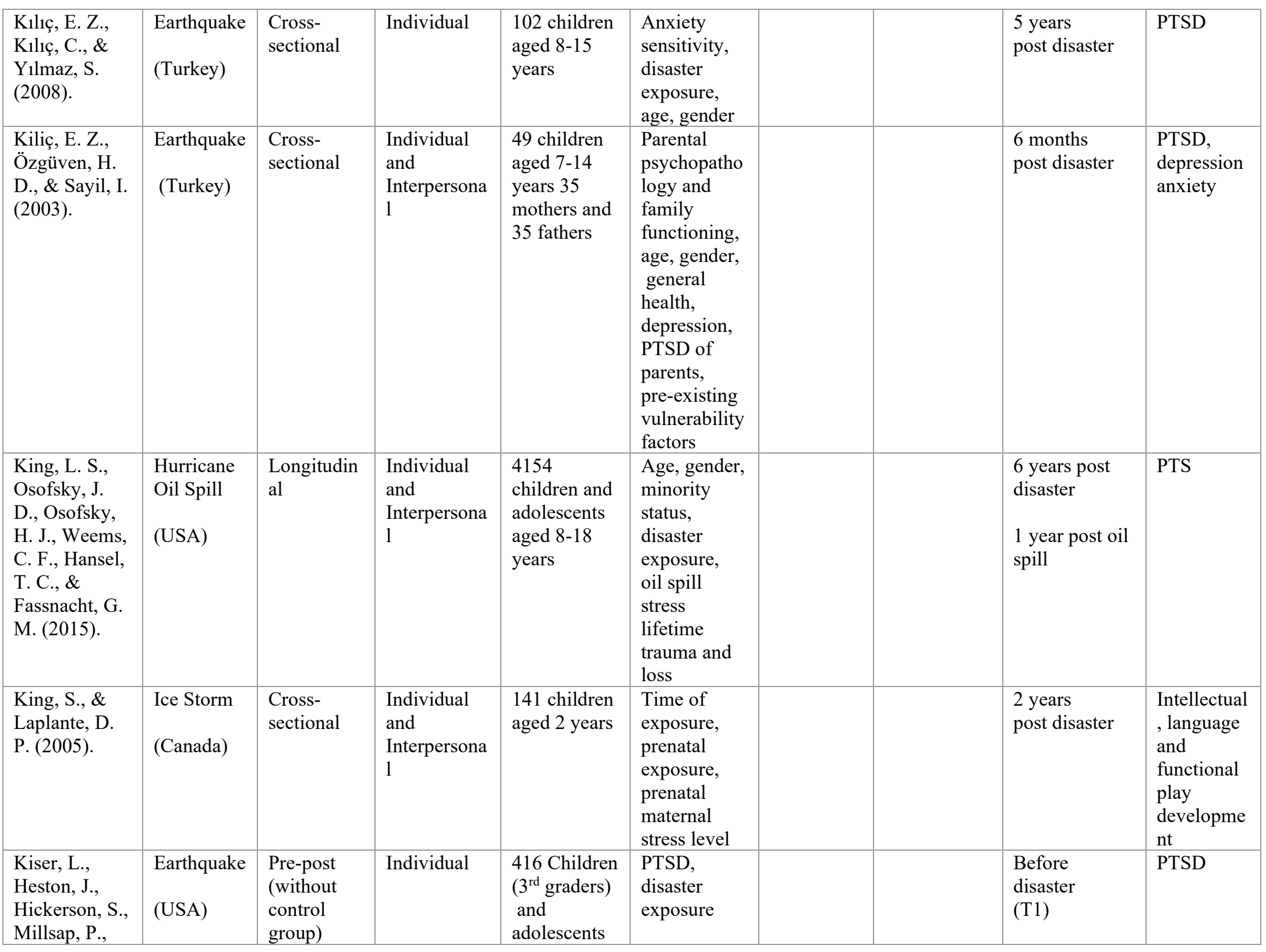




\section{RESILIENCE IN CHILDREN AFTER NATURAL DISASTERS}

\begin{tabular}{|c|c|c|c|c|c|c|c|c|}
\hline $\begin{array}{l}\text { Nunn, W., \& } \\
\text { Pruitt, D. } \\
\text { (1993). }\end{array}$ & & & & $\begin{array}{l}\left(10^{\text {th }}\right. \\
\text { graders })\end{array}$ & & & $\begin{array}{l}\text { 6-8 weeks post } \\
\text { disaster } \\
\text { (T2) }\end{array}$ & \\
\hline $\begin{array}{l}\text { Knight, L. } \\
\text { A., \& } \\
\text { Sullivan, M. } \\
\text { A. (2006). }\end{array}$ & $\begin{array}{l}\text { Tornado } \\
\text { (USA) }\end{array}$ & $\begin{array}{l}\text { Cross- } \\
\text { sectional }\end{array}$ & Individual & $\begin{array}{l}52 \text { children. } \\
\text { aged } 8-12 \\
\text { years }\end{array}$ & $\begin{array}{l}\text { Disaster } \\
\text { exposure, } \\
\text { trauma } \\
\text { attribution }\end{array}$ & & $\begin{array}{l}12 \text { Months } \\
\text { post disaster }\end{array}$ & PTSD \\
\hline $\begin{array}{l}\text { Kolaitis, G., } \\
\text { Kotsopoulos, } \\
\text { J., Tsiantis, J., } \\
\text { Haritaki, S., } \\
\text { Rigizou, F., } \\
\text { Zacharaki, L., } \\
\text {... \& } \\
\text { Liakopoulou, } \\
\text { M. } \\
(2003) .\end{array}$ & $\begin{array}{l}\text { Earthquake } \\
\text { (Greece) }\end{array}$ & $\begin{array}{l}\text { Cross- } \\
\text { sectional }\end{array}$ & Individual & $\begin{array}{l}163 \text { children, } \\
4^{\text {th }}-5^{\text {th }}-6^{\text {th }} \\
\text { grades }\end{array}$ & $\begin{array}{l}\text { Disaster } \\
\text { exposure, } \\
\text { gender, } \\
\text { native vs. } \\
\text { immigrant } \\
\text { status, where } \\
\text { the child was } \\
\text { at the time } \\
\text { of the } \\
\text { earthquake, } \\
\text { child } \\
\text { injured, } \\
\text { others } \\
\text { injured, } \\
\text { property } \\
\text { damage, } \\
\text { previous } \\
\text { disaster } \\
\text { exposure, } \\
\text { age, } \\
\text { education } \\
\text { level of the } \\
\text { father, } \\
\text { mother's } \\
\text { reaction }\end{array}$ & & $\begin{array}{l}6 \text { Months post } \\
\text { disaster }\end{array}$ & $\begin{array}{l}\text { PTSD } \\
\text { Depression } \\
\text { Anxiety }\end{array}$ \\
\hline $\begin{array}{l}\text { Kopala- } \\
\text { Sibley, D. C., } \\
\text { Danzig, A. P., } \\
\text { Kotov, R., } \\
\text { Bromet, E. J., } \\
\text { Carlson, G. } \\
\text { A., Olino, T. } \\
\text { M., ... \& }\end{array}$ & $\begin{array}{l}\text { Hurricane } \\
\text { (USA) }\end{array}$ & $\begin{array}{l}\text { Pre-post } \\
\text { (without } \\
\text { control } \\
\text { group) }\end{array}$ & Individual & $\begin{array}{l}332 \text { children } \\
\text { aged } 3 \text { years }\end{array}$ & $\begin{array}{l}\text { Disaster } \\
\text { exposure, } \\
\text { temperament }\end{array}$ & $\begin{array}{l}\text { Early } \\
\text { childhood } \\
\text { temperament } \\
\text { negative } \\
\text { emotionality } \\
\text { and its facets } \\
\text { Temperamen } \\
\text { t }\end{array}$ & $\begin{array}{l}7 \text { years } \\
\text { pre disaster } \\
6 \text { years post } \\
\text { disaster }\end{array}$ & $\begin{array}{l}\text { Depression } \\
\text { and } \\
\text { anxiety } \\
\text { symptoms }\end{array}$ \\
\hline
\end{tabular}




\begin{tabular}{|c|c|c|c|c|c|c|c|c|c|}
\hline $\begin{array}{l}\text { Klein, D. N. } \\
(2016) .\end{array}$ & & & & & & & & & \\
\hline $\begin{array}{l}\text { Kreuger, L., } \\
\text { \& Stretch, J. } \\
(2003) .\end{array}$ & $\begin{array}{l}\text { Flood } \\
\text { (USA) }\end{array}$ & $\begin{array}{l}\text { Experiment } \\
\text { al } \\
\text { (without } \\
\text { control } \\
\text { group) }\end{array}$ & Individual & $\begin{array}{l}3876 \\
\text { children and } \\
\text { adolescents } \\
4^{\text {th }}-5^{\text {th }} \text { and } \\
12^{\text {th }} \text { grades }\end{array}$ & $\begin{array}{l}\text { Disaster } \\
\text { exposure, } \\
\text { stayed/ } \\
\text { evacuated } \\
\text { residence, } \\
\text { ability of } \\
\text { family to } \\
\text { recover }\end{array}$ & & & & PTSD \\
\hline $\begin{array}{l}\text { Kronenberg, } \\
\text { M. E., Hansel, } \\
\text { T. C., } \\
\text { Brennan, A. } \\
\text { M., Osofsky, } \\
\text { H. J., } \\
\text { Osofsky, J. } \\
\text { D., \& } \\
\text { Lawrason, B. } \\
(2010) \text {. }\end{array}$ & $\begin{array}{l}\text { Hurricane } \\
\text { (USA) }\end{array}$ & $\begin{array}{l}\text { Longitudin } \\
\text { al }\end{array}$ & Individual & $\begin{array}{l}387 \text { children } \\
\text { aged 9-18 } \\
\text { years }\end{array}$ & $\begin{array}{l}\text { Time, } \\
\text { disaster } \\
\text { exposure, } \\
\text { gender, } \\
\text { age, } \\
\text { life stressor }\end{array}$ & & & $\begin{array}{l}2-3 \text { years } \\
\text { post disaster }\end{array}$ & $\begin{array}{l}\text { Outcome } \\
\text { Trajectorie } \\
\mathrm{s} \\
\text { Depression } \\
\text { PTSD }\end{array}$ \\
\hline $\begin{array}{l}\text { Kroska, E. B., } \\
\text { O’Hara, M. } \\
\text { W., Elgbeili, } \\
\text { G., Hart, K. } \\
\text { J., Laplante, } \\
\text { D. P., } \\
\text { Dancause, K. } \\
\text { N., \& King, S. } \\
(2018) .\end{array}$ & $\begin{array}{l}\text { Flood } \\
\text { (USA) }\end{array}$ & $\begin{array}{l}\text { Cross- } \\
\text { sectional }\end{array}$ & $\begin{array}{l}\text { Individual } \\
\text { Interpersona } \\
1\end{array}$ & $\begin{array}{l}103 \text { mothers } \\
\text { who were } \\
\text { pregnant } \\
\text { when } \\
\text { disastrous } \\
\text { occurred }\end{array}$ & $\begin{array}{l}\text { Pprenatal } \\
\text { maternal } \\
\text { stress, } \\
\text { cognitive } \\
\text { appraisal of } \\
\text { the flood } \\
\text { consequence } \\
\text { s, } \\
\text { timing of in } \\
\text { utero } \\
\text { exposure } \\
\text { maternal } \\
\text { depression }\end{array}$ & $\begin{array}{l}\text { Offspring } \\
\text { birthweigh } \\
\mathrm{t}\end{array}$ & $\begin{array}{l}\text { Social } \\
\text { support }\end{array}$ & $\begin{array}{l}30 \text { months } \\
\text { post disaster }\end{array}$ & $\begin{array}{l}\text { Childhood } \\
\text { body mass } \\
\text { index }\end{array}$ \\
\hline
\end{tabular}




\begin{tabular}{|c|c|c|c|c|c|c|c|}
\hline $\begin{array}{l}\text { Küçükoğlu, } \\
\text { S., Yıldırım, } \\
\text { N., \& Dursun, } \\
\text { O. B. (2015). }\end{array}$ & $\begin{array}{l}\text { Earthquake } \\
\text { (Turkey) }\end{array}$ & $\begin{array}{l}\text { Cross- } \\
\text { sectional }\end{array}$ & Individual & $\begin{array}{l}304 \text { children } \\
\text { aged } 7-12 \\
\text { years }\end{array}$ & $\begin{array}{l}\text { Disaster } \\
\text { exposure, } \\
\text { demographic } \\
\text { characteristi } \\
\text { cs }\end{array}$ & $\begin{array}{l}\text { Within the } \\
3 \text { months } \\
\text { post disaster }\end{array}$ & PTSS \\
\hline $\begin{array}{l}\text { Kujawa, A., } \\
\text { Hajcak, G., } \\
\text { Danzig, A. P., } \\
\text { Black, S. R., } \\
\text { Bromet, E. J., } \\
\text { Carlson, G. } \\
\text { A., ... \& } \\
\text { Klein, D. N. } \\
(2016) .\end{array}$ & $\begin{array}{l}\text { Hurricane } \\
\text { (USA) }\end{array}$ & $\begin{array}{l}\text { Longitudin } \\
\text { al }\end{array}$ & Individual & $\begin{array}{l}260 \text { children } \\
\text { aged } 9-12 \\
\text { years }\end{array}$ & $\begin{array}{l}\text { Late positive } \\
\text { potential, } \\
\text { electroencep } \\
\text { halogram, } \\
\text { hurricane- } \\
\text { related } \\
\text { stress, } \\
\text { prehurricane } \\
\text { internalizing } \\
\text { and } \\
\text { externalizing } \\
\text { symptoms } \\
\text { and child, } \\
\text { gender }\end{array}$ & $\begin{array}{l}8 \text { weeks } \\
\text { post disaster } \\
8 \text { months } \\
\text { post disaster }\end{array}$ & $\begin{array}{l}\text { Children's } \\
\text { internalizin } \\
\mathrm{g} \\
\text { and } \\
\text { externalizi } \\
\text { ng } \\
\text { symptoms }\end{array}$ \\
\hline $\begin{array}{l}\text { Kumar, M., \& } \\
\text { Fonagy, P. } \\
(2012) .\end{array}$ & $\begin{array}{l}\text { Earthquake } \\
\text { (India) }\end{array}$ & $\begin{array}{l}\text { Experiment } \\
\text { al } \\
\text { (with } \\
\text { control } \\
\text { group }\end{array}$ & Individual & $\begin{array}{l}\text { Exposed } \\
\text { children } \\
\text { sample } \\
(\mathrm{n}=48), \\
\text { riots sample } \\
(\mathrm{n}=37) \\
\text { control } \\
\text { sample } \\
(\mathrm{n}=42) \\
\\
3 \text { age } \\
\text { groups: } \\
8-9 \text { years } \\
10-11 \text { years } \\
12-13 \text { years } \\
\text { Above } 14 \\
\text { years }\end{array}$ & $\begin{array}{l}\text { Trauma } \\
\text { groups }\end{array}$ & & $\begin{array}{l}\text { Attachmen } \\
\text { t styles } \\
\text { psychologi } \\
\text { cal } \\
\text { adjustment }\end{array}$ \\
\hline
\end{tabular}




\begin{tabular}{|c|c|c|c|c|c|c|c|}
\hline $\begin{array}{l}\text { Kumar, M., \& } \\
\text { Fonagy, P. } \\
(2013) .\end{array}$ & $\begin{array}{l}\text { Earthquake } \\
\text { (India) }\end{array}$ & $\begin{array}{l}\text { Experiment } \\
\text { al (with } \\
\text { control } \\
\text { group) }\end{array}$ & Individual & $\begin{array}{l}299 \text { children } \\
\text { aged } 8-15 \\
\text { years } \\
\text { Three } \\
\text { groups: } \\
\text { highly } \\
\text { exposed } \\
\text { earthquake } \\
\text { sites (n = } \\
\text { 128) } \\
\text { and riot sites } \\
\text { (n = 171) } \\
\text { Control } \\
\text { sample of } \\
351 \\
\text { no-trauma } \\
\text { exposed }\end{array}$ & $\begin{array}{l}\text { Type of } \\
\text { disaster }\end{array}$ & $\begin{array}{l}3.4 \text { years } \\
\text { post disaster }\end{array}$ & $\begin{array}{l}\text { Adjustmen } \\
\mathrm{t} \\
\text { difficulties } \\
\text { PTSD }\end{array}$ \\
\hline $\begin{array}{l}\text { Kuwabara, H., } \\
\text { Araki, T., } \\
\text { Yamasaki, S., } \\
\text { Ando, S., } \\
\text { Kano, Y., \& } \\
\text { Kasai, K. } \\
(2015) .\end{array}$ & $\begin{array}{l}\text { Tsunami } \\
\text { (Japan) }\end{array}$ & $\begin{array}{l}\text { Cross- } \\
\text { sectional }\end{array}$ & Individual & $\begin{array}{l}1102 \\
\text { children and } \\
1157 \\
\text { adolescents }\end{array}$ & $\begin{array}{l}\text { Age, gender, } \\
\text { high impact } \\
\text { group, } \\
\text { lower- } \\
\text { impact } \\
\text { group (effect } \\
\text { only for } \\
\text { PTSS) }\end{array}$ & $\begin{array}{l}6 \text { weeks } \\
\text { post disaster }\end{array}$ & $\begin{array}{l}\text { PTSS } \\
\text { PTSD, } \\
\text { depression }\end{array}$ \\
\hline $\begin{array}{l}\text { La Greca, A. } \\
\text { M., Danzi, B. } \\
\text { A., \& Chan, } \\
\text { S. F. (2017). }\end{array}$ & $\begin{array}{l}\text { Hurricane } \\
\text { (USA) }\end{array}$ & $\begin{array}{l}\text { Cross- } \\
\text { sectional }\end{array}$ & Individual & $\begin{array}{l}327 \text { children } \\
\text { aged } 7-11 \\
\text { years }\end{array}$ & $\begin{array}{l}\text { Gender, } \\
\text { ethnicity, } \\
\text { age, } \\
\text { dsm-IV, } \\
\text { dsm-V } \\
\text { and ICD-11 }\end{array}$ & $\begin{array}{l}8 \text { months } \\
\text { post disaster }\end{array}$ & $\begin{array}{l}\text { PTSD, } \\
\text { anxiety } \\
\text { and } \\
\text { depression }\end{array}$ \\
\hline
\end{tabular}




\begin{tabular}{|c|c|c|c|c|c|c|c|c|c|}
\hline $\begin{array}{l}\text { Auslander, B. } \\
\text { B., \& Short, } \\
\text { M. A. (2013). }\end{array}$ & & & $\begin{array}{l}\text { Interpersona } \\
1\end{array}$ & & $\begin{array}{l}\text { disaster } \\
\text { exposure, } \\
\text { hurricane- } \\
\text { related } \\
\text { stressors, } \\
\text { social } \\
\text { support, } \\
\text { genetic } \\
\text { markers }\end{array}$ & & & & \\
\hline $\begin{array}{l}\text { La Greca, A. } \\
\text { M., Lai, B. S., } \\
\text { Llabre, M. } \\
\text { M., } \\
\text { Silverman, W. } \\
\text { K., Vernberg, } \\
\text { E. M., \& } \\
\text { Prinstein, M. } \\
\text { J. } \\
(2013) .\end{array}$ & $\begin{array}{l}\text { Hurricane } \\
\text { (USA) }\end{array}$ & $\begin{array}{l}\text { Longitudin } \\
\text { al }\end{array}$ & $\begin{array}{l}\text { Individual } \\
\text { and } \\
\text { Interpersona } \\
1\end{array}$ & $\begin{array}{l}568 \text { children } \\
\text { aged } 9 \text { years }\end{array}$ & $\begin{array}{l}\text { Disaster } \\
\text { exposure, } \\
\text { stressors, } \\
\text { gender, } \\
\text { social } \\
\text { support, } \\
\text { coping, } \\
\text { general } \\
\text { anxiety }\end{array}$ & & & $\begin{array}{l}3 \text { months } \\
\text { post disaster } \\
\text { (T1) } \\
7 \text { months } \\
\text { post disaster } \\
\text { (T2) } \\
10 \text { months } \\
\text { post disaster } \\
\text { (T3) }\end{array}$ & PTSS \\
\hline $\begin{array}{l}\text { La Greca, A. } \\
\text { M., } \\
\text { Silverman, W. } \\
\text { K., \& } \\
\text { Wasserstein, } \\
\text { S. B. } \\
\text { (1998). }\end{array}$ & $\begin{array}{l}\text { Hurricane } \\
\text { (USA) }\end{array}$ & $\begin{array}{l}\text { Longitudin } \\
\text { al }\end{array}$ & $\begin{array}{l}\text { Individual, } \\
\text { Interpersona } \\
1 \text { and } \\
\text { Intergroup }\end{array}$ & $\begin{array}{l}92 \text { children } \\
\text { of } 4^{\text {th }}, 5^{\text {th }} \text { and } \\
6^{\text {th }} \text { grades }\end{array}$ & $\begin{array}{l}\text { Disaster } \\
\text { exposure: } \\
\text { life threat } \\
\text { during the } \\
\text { event, } \\
\text { loss/disrupti } \\
\text { on following } \\
\text { the event }\end{array}$ & $\begin{array}{l}\text { Efforts to } \\
\text { Process } \\
\text { and Cope } \\
\text { with } \\
\text { Events }\end{array}$ & 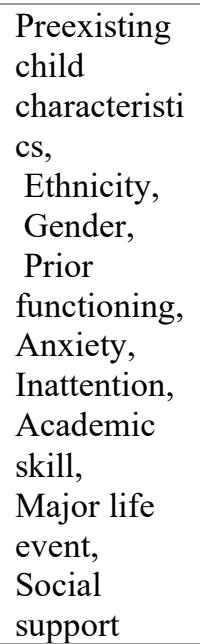 & $\begin{array}{l}15 \text { months } \\
\text { Pre-disaster } \\
\text { (T1), } \\
3 \text { months } \\
\text { post disaster } \\
\text { (T2), } \\
7 \text { months } \\
\text { post disaster } \\
\text { (T3) }\end{array}$ & PTS \\
\hline
\end{tabular}




\begin{tabular}{|c|c|c|c|c|c|c|c|c|}
\hline $\begin{array}{l}\text { Jaccard, J. } \\
\text { (2010). }\end{array}$ & & & & $\begin{array}{l}\text { aged } \\
M=8.74 \\
\text { years } \\
245 \text { children } \\
\text { (T2) }\end{array}$ & & & $\begin{array}{l}21 \text { months } \\
\text { post disaster } \\
\text { (T2) }\end{array}$ & \\
\hline $\begin{array}{l}\text { La Greca, A. } \\
\text { M., } \\
\text { Silverman, W. } \\
\text { K., Vernberg, } \\
\text { E. M., \& } \\
\text { Prinstein, M. } \\
\text { J. } \\
\text { (1996). }\end{array}$ & $\begin{array}{l}\text { Hurricane } \\
\text { (USA) }\end{array}$ & $\begin{array}{l}\text { Longitudin } \\
\text { al }\end{array}$ & Individual & $\begin{array}{l}442 \\
\text { Children, } \\
3^{\text {rd }}-5^{\text {th }} \\
\text { grades }\end{array}$ & $\begin{array}{l}\text { Disaster } \\
\text { exposure, } \\
\text { preexisting } \\
\text { child } \\
\text { characteristi } \\
\text { cs (e.g.., } \\
\text { ethnicity), } \\
\text { characteristi } \\
\text { cs of the } \\
\text { post disaster } \\
\text { recovery, } \\
\text { environment } \\
\text { : major life } \\
\text { stressors, } \\
\text { social } \\
\text { support }\end{array}$ & Coping & $\begin{array}{l}3 \text { months } \\
\text { post disaster } \\
\text { (T1) } \\
7 \text { months } \\
\text { post disaster } \\
\text { (T2) } \\
10 \text { months } \\
\text { post disaster } \\
\text { (T3) }\end{array}$ & PTSS \\
\hline $\begin{array}{l}\text { Lack, C. W., } \\
\text { Sullivan, M. } \\
\text { A., (2008). }\end{array}$ & $\begin{array}{l}\text { Tornado } \\
\text { (USA) }\end{array}$ & $\begin{array}{l}\text { Cross- } \\
\text { sectional }\end{array}$ & Individual & $\begin{array}{l}102 \text { children } \\
\text { aged } 8-12 \\
\text { years and } \\
96 \text { parents }\end{array}$ & $\begin{array}{l}\text { Initial } \\
\text { disaster } \\
\text { exposure, } \\
\text { attributions } \\
\text { about the } \\
\text { disaster, } \\
\text { coping style }\end{array}$ & & $\begin{array}{l}13 \text { months } \\
\text { post disaster }\end{array}$ & $\begin{array}{l}\text { Long-term } \\
\text { posttrauma } \\
\text { tic } \\
\text { distress }\end{array}$ \\
\hline $\begin{array}{l}\text { Lai, B. S., } \\
\text { Kelley M. L., } \\
\text { Harrison, K. } \\
\text { M., } \\
\text { Thompson, J. } \\
\text { E., \& Brown, } \\
\text { S-S. } \\
(2014) .\end{array}$ & $\begin{array}{l}\text { Hurricane } \\
\text { (USA) }\end{array}$ & $\begin{array}{l}\text { Longitudin } \\
\text { al }\end{array}$ & $\begin{array}{l}\text { Individual } \\
\text { and } \\
\text { Interpersona } \\
1\end{array}$ & $\begin{array}{l}353 \text { mother- } \\
\text { child dyads } \\
\text { aged } 8-15 \\
\text { years }\end{array}$ & $\begin{array}{l}\text { Levels of } \\
\text { disaster } \\
\text { stressor } \\
\text { agreement } \\
\text { among } \\
\text { mother- } \\
\text { child dyads, } \\
\text { social } \\
\text { support from } \\
\text { parents }\end{array}$ & & $\begin{array}{l}\text { 3-7 months } \\
\text { post disaster } \\
\text { (T1) } \\
14-17 \text { months } \\
\text { post disaster } \\
\text { (T2) }\end{array}$ & $\begin{array}{l}\text { PTS in } \\
\text { children } \\
\text { PTS in } \\
\text { mothers }\end{array}$ \\
\hline
\end{tabular}




\begin{tabular}{|c|c|c|c|c|c|c|c|c|}
\hline & & & & & $\begin{array}{l}\text { or a } \\
\text { classmate/fri } \\
\text { end } \\
\text { disaster } \\
\text { related } \\
\text { experiences, } \\
\text { actual life } \\
\text { threat }\end{array}$ & & & \\
\hline $\begin{array}{l}\text { Lai, B. S., La } \\
\text { Greca, A. M., } \\
\text { \& Llabre, M. } \\
\text { M. (2014). }\end{array}$ & $\begin{array}{l}\text { Hurricane } \\
\text { (USA) }\end{array}$ & $\begin{array}{l}\text { Cross- } \\
\text { sectional }\end{array}$ & Individual & $\begin{array}{l}204 \text { children } \\
\text { aged } 7-11 \\
\text { years }\end{array}$ & $\begin{array}{l}\text { Impact of a } \\
\text { natural } \\
\text { disaster, } \\
\text { disaster } \\
\text { exposure, } \\
\text { recovery } \\
\text { stressors, } \\
\text { gender }\end{array}$ & $\begin{array}{l}\text { PTS } \\
\text { symptoms } \\
\text { activity }\end{array}$ & $\begin{array}{l}8 \text { months } \\
\text { post disaster }\end{array}$ & $\begin{array}{l}\text { PTS } \\
\text { symptoms } \\
\text { Sedentary } \\
\text { activity }\end{array}$ \\
\hline $\begin{array}{l}\text { Lai, B. S., La } \\
\text { Greca, A. M., } \\
\text { Auslander, B. } \\
\text { A., \& Short, } \\
\text { M. B. } \\
(2013) .\end{array}$ & $\begin{array}{l}\text { Hurricane } \\
\text { (USA) }\end{array}$ & $\begin{array}{l}\text { Longitudin } \\
\text { al }\end{array}$ & Individual & $\begin{array}{l}277 \text { children } \\
\text { (between } \\
8.70 \text { and } \\
9.40 \text { years) }\end{array}$ & $\begin{array}{l}\text { Disaster } \\
\text { exposure, } \\
\text { recovery } \\
\text { stressors, } \\
\text { time }\end{array}$ & & $\begin{array}{l}8 \text { months } \\
\text { post disaster } \\
\text { (T1) } \\
15 \text { months } \\
\text { post disaster } \\
\text { (T2) }\end{array}$ & $\begin{array}{l}\text { PTSD and } \\
\text { depression }\end{array}$ \\
\hline $\begin{array}{l}\text { Lai, B. S., } \\
\text { Osborne, M. } \\
\text { C., Piscitello, } \\
\text { J., Self- } \\
\text { Brown, S., \& } \\
\text { Kelley, M. L. } \\
(2018) .\end{array}$ & $\begin{array}{l}\text { Hurricane } \\
\text { (USA) }\end{array}$ & $\begin{array}{l}\text { Longitudin } \\
\text { al }\end{array}$ & $\begin{array}{l}\text { Individual } \\
\text { and } \\
\text { Interpersona } \\
1\end{array}$ & $\begin{array}{l}426 \text { children } \\
\text { aged } 8-16 \\
\text { years }\end{array}$ & $\begin{array}{l}\text { Social } \\
\text { support, } \\
\text { time }\end{array}$ & & $\begin{array}{l}\text { 3-7 } \\
\text { months (T1) } \\
13-17 \\
\text { months (T2) } \\
\text { 19-22 } \\
\text { months (T3) }\end{array}$ & PTSS \\
\hline
\end{tabular}




\section{RESILIENCE IN CHILDREN AFTER NATURAL DISASTERS}

\begin{tabular}{|c|c|c|c|c|c|c|c|}
\hline & & & & & & $\begin{array}{l}25-27 \\
\text { months (T4) }\end{array}$ & \\
\hline $\begin{array}{l}\text { Lai, B. S., } \\
\text { Tiwari, A., } \\
\text { Beaulieu, B. } \\
\text { A., Self- } \\
\text { Brown, S., \& } \\
\text { Kelley, M. L. } \\
(2015) .\end{array}$ & $\begin{array}{l}\text { Hurricane } \\
\text { (USA) }\end{array}$ & $\begin{array}{l}\text { Longitudin } \\
\text { al }\end{array}$ & $\begin{array}{l}\text { Individual } \\
\text { and } \\
\text { Interpersona } \\
1\end{array}$ & $\begin{array}{l}283 \text { mother- } \\
\text { child dyads } \\
\text { aged } 8-15 \\
\text { years }\end{array}$ & $\begin{array}{l}\text { Mothers } \\
\text { posttraumati } \\
\text { c stress } \\
\text { symptoms, } \\
\text { disaster } \\
\text { exposure, } \\
\text { traumatic } \\
\text { life events, } \\
\text { social } \\
\text { support, } \\
\text { time 1 }\end{array}$ & $\begin{array}{l}\text { 3-7 months } \\
\text { post disaster } \\
\text { (T1) } \\
\text { 13-17 months } \\
\text { post disaster } \\
\text { (T2) } \\
\text { 19-22 months } \\
\text { post disaster } \\
\text { (T3) } \\
25-27 \\
\text { months } \\
\text { post disaster }\end{array}$ & $\begin{array}{l}\text { PTSS } \\
\text { depression } \\
\text { anxiety }\end{array}$ \\
\hline $\begin{array}{l}\text { Laor, N., } \\
\text { Wolmer, L., } \\
\text { Kora, M., } \\
\text { Yucel, D., } \\
\text { Spirman, S., } \\
\text { \& Yazgan, Y. } \\
(2002) .\end{array}$ & $\begin{array}{l}\text { Earthquake } \\
\text { (Turkey) }\end{array}$ & $\begin{array}{l}\text { Cross- } \\
\text { sectional }\end{array}$ & Individual & $\begin{array}{l}303 \text { children } \\
\text { aged } 8 \text { years }\end{array}$ & $\begin{array}{l}\text { Disaster } \\
\text { exposure, } \\
\text { gender, } \\
\text { age, } \\
\text { past trauma } \\
\text { experiences, } \\
\text { predisaster } \\
\text { functioning, } \\
\text { grief, } \\
\text { dissociation }\end{array}$ & $\begin{array}{l}4 \text { months } \\
\text { post disaster }\end{array}$ & PTSD \\
\hline $\begin{array}{l}\text { Laplante, D. } \\
\text { P., Brunet, A., } \\
\text { Schmitz, N., } \\
\text { Ciampi, A., \& } \\
\text { King, S. } \\
(2008) .\end{array}$ & $\begin{array}{l}\text { Ice Storm } \\
\text { (Canada) }\end{array}$ & $\begin{array}{l}\text { Cross- } \\
\text { sectional }\end{array}$ & $\begin{array}{l}\text { Individual } \\
\text { and } \\
\text { Interpersona } \\
1\end{array}$ & $\begin{array}{l}89 \text { mothers- } \\
\text { child dyads } \\
\text { aged } M=5.5 \\
\text { years }\end{array}$ & 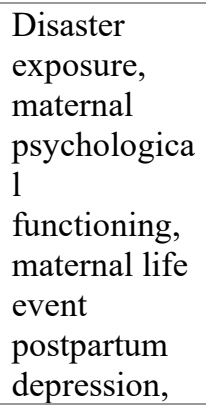 & $\begin{array}{l}5 \text { years } \\
\text { post disaster }\end{array}$ & $\begin{array}{l}\text { Intellectual } \\
\text { and } \\
\text { language } \\
\text { performan } \\
\text { ce of } \\
\text { children }\end{array}$ \\
\hline
\end{tabular}




\begin{tabular}{|c|c|c|c|c|c|c|c|}
\hline & & & & & $\begin{array}{l}\text { time of utero } \\
\text { exposure, } \\
\text { demographic } \\
\text { factors }\end{array}$ & & \\
\hline $\begin{array}{l}\text { Laplante, D. } \\
\text { P., Zelazo, P. } \\
\text { R., Brunet, } \\
\text { A., \& King, S. } \\
(2007) .\end{array}$ & $\begin{array}{l}\text { Ice Storm } \\
\text { (Canada) }\end{array}$ & $\begin{array}{l}\text { Cross- } \\
\text { sectional }\end{array}$ & $\begin{array}{l}\text { Individual } \\
\text { and } \\
\text { Interpersona } \\
1\end{array}$ & $\begin{array}{l}52 \text { children } \\
\text { aged } 2 \text { years }\end{array}$ & $\begin{array}{l}\text { Prenatal } \\
\text { maternal } \\
\text { stress, } \\
\text { trimester of } \\
\text { pregnancy, } \\
\text { maternal and } \\
\text { pregnancy } \\
\text { factors, } \\
\text { maternal } \\
\text { anxiety and } \\
\text { depression, } \\
\text { parental } \\
\text { socioeconom } \\
\text { ic status }\end{array}$ & $\begin{array}{l}2 \text { years } \\
\text { post disaster }\end{array}$ & $\begin{array}{l}\text { Functional } \\
\text { play }\end{array}$ \\
\hline $\begin{array}{l}\text { Lee, O. } \\
(1999) \text {. }\end{array}$ & $\begin{array}{l}\text { Hurricane } \\
\text { (USA) }\end{array}$ & $\begin{array}{l}\text { Cross- } \\
\text { sectional }\end{array}$ & $\begin{array}{l}\text { Individual } \\
\text { and } \\
\text { Intergroup }\end{array}$ & $\begin{array}{l}1274 \text { and } 5^{\text {th }} \\
\text { grades }\end{array}$ & $\begin{array}{l}\text { Ethnicity, } \\
\text { socioeconom } \\
\text { ic status, } \\
\text { gender }\end{array}$ & $\begin{array}{l}18 \text { months } \\
\text { post hurricane }\end{array}$ & $\begin{array}{l}\text { Science } \\
\text { knowledge } \\
\text { world } \\
\text { views, } \\
\text { informatio } \\
\text { n } \\
\text { sources }\end{array}$ \\
\hline $\begin{array}{l}\text { Li, G., Wang, } \\
\text { L., Cao, C., } \\
\text { Fang, R., Cao, } \\
\text { X., Chen, C., } \\
\text { Elhai, J. D., \& } \\
\text { Hall, B. J. } \\
\text { (2019) }\end{array}$ & $\begin{array}{l}\text { Earthquake } \\
\text { (China) }\end{array}$ & $\begin{array}{l}\text { Cross- } \\
\text { sectional }\end{array}$ & $\begin{array}{l}\text { Individual, } \\
\text { Intergroup }\end{array}$ & $\begin{array}{l}13.438 \\
\text { children 6- } \\
18 \text { years old }\end{array}$ & $\begin{array}{l}\text { Trauma } \\
\text { exposure } \\
\text { Quality of } \\
\text { life } \\
\text { Sex } \\
\text { Age } \\
\text { Ethnicity }\end{array}$ & & $\begin{array}{l}\text { PTSD } \\
\text { Executive } \\
\text { dysfunctio } \\
\mathrm{n} \\
\text { symptoms }\end{array}$ \\
\hline $\begin{array}{l}\text { Li, X., Huang, } \\
\text { X., Tan, H., } \\
\text { Liu, A., Zhou, } \\
\text { J., \& Yang, T. } \\
(2010) .\end{array}$ & $\begin{array}{l}\text { Flood } \\
\text { (China) }\end{array}$ & $\begin{array}{l}\text { Cross- } \\
\text { sectional }\end{array}$ & $\begin{array}{l}\text { Individual } \\
\text { and } \\
\text { Interpersona } \\
1\end{array}$ & $\begin{array}{l}3698 \\
\text { families } \\
\text { Children } \\
\text { aged 7-15 } \\
\text { years }\end{array}$ & $\begin{array}{l}\text { PTSD in } \\
\text { parents, } \\
\text { flood } \\
\text { exposure } \\
\text { level of the } \\
\text { children }\end{array}$ & $\begin{array}{l}18 \text { months } \\
\text { post disaster }\end{array}$ & $\begin{array}{l}\text { Children } \\
\text { PTSD }\end{array}$ \\
\hline
\end{tabular}




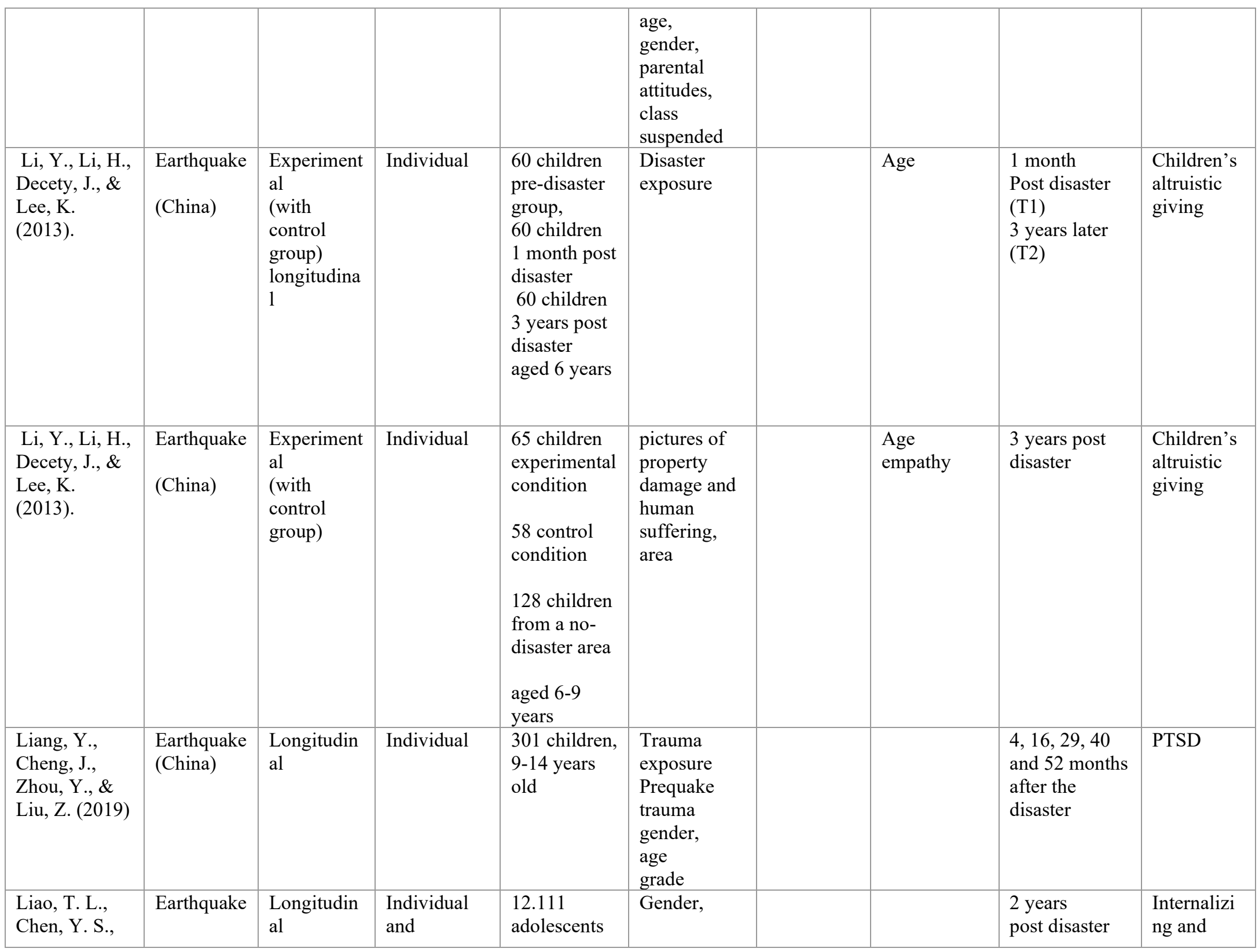




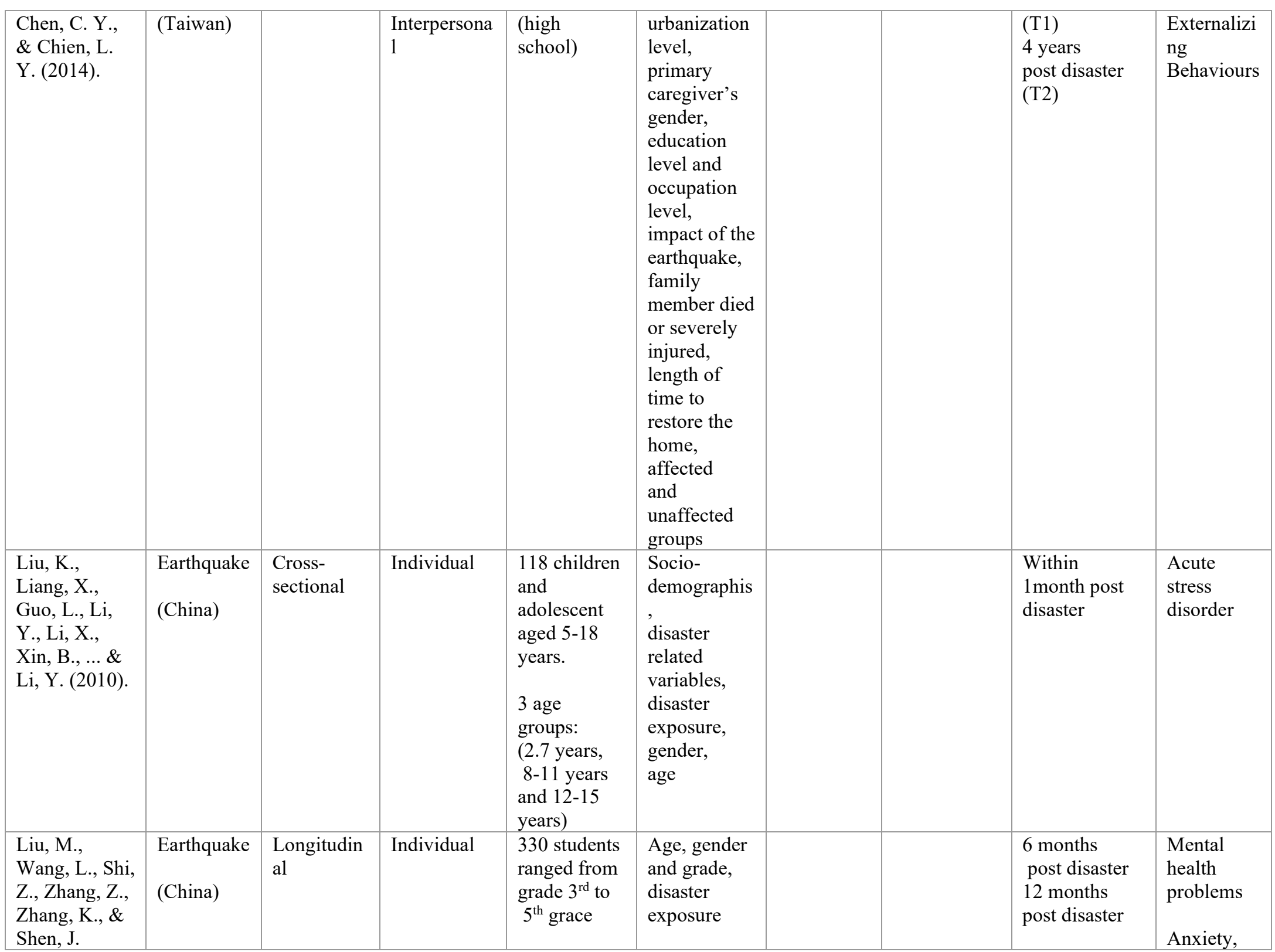




\begin{tabular}{|c|c|c|c|c|c|c|c|c|}
\hline (2011). & & & & & & & & $\begin{array}{l}\text { depression } \\
\text { and PTSD }\end{array}$ \\
\hline $\begin{array}{l}\text { Liu, X., Yang, } \\
\text { H., Tang, B., } \\
\text { Liu, Y., \& } \\
\text { Zhang, L. } \\
\text { (2017). }\end{array}$ & $\begin{array}{l}\text { Earthquake } \\
\text { (China) }\end{array}$ & $\begin{array}{l}\text { Cross- } \\
\text { sectional }\end{array}$ & $\begin{array}{l}\text { Individual, } \\
\text { Interpersona } \\
1 \text { and } \\
\text { Intergroup }\end{array}$ & $\begin{array}{l}591 \text { children } \\
\text { and } \\
\text { adolescent } \\
\text { aged } 12-16 \\
\text { years }\end{array}$ & $\begin{array}{l}\text { Gender, age, } \\
\text { ethnicity, } \\
\text { religious } \\
\text { faith, } \\
\text { chronic } \\
\text { disease } \\
\text { history, } \\
\text { residential } \\
\text { area, family } \\
\text { resident } \\
\text { population, } \\
\text { main } \\
\text { source of } \\
\text { income for } \\
\text { the family, } \\
\text { disaster } \\
\text { related } \\
\text { experiences, } \\
\text { psychologica } \\
\text { l counselling } \\
\text { and training }\end{array}$ & & $\begin{array}{l}6 \text { years } \\
\text { post disaster }\end{array}$ & $\begin{array}{l}\text { Physical } \\
\text { and mental } \\
\text { health } \\
\text { status }\end{array}$ \\
\hline $\begin{array}{l}\text { Lochman, J. } \\
\text { E., Vernberg, } \\
\text { E., Powell, N. } \\
\text { P., Boxmeyer, } \\
\text { C. L., Jarrett, } \\
\text { M., } \\
\text { McDonald, } \\
\text { K., ... \& } \\
\text { Kassing, F. } \\
\text { (2016) }\end{array}$ & $\begin{array}{l}\text { Tornado } \\
\text { (USA) }\end{array}$ & $\begin{array}{l}\text { Pre post } \\
\text { (without } \\
\text { control } \\
\text { group) } \\
\text { and } \\
\text { longitudina } \\
1\end{array}$ & Individual & $\begin{array}{l}360 \text { children } \\
\text { aged } 9-11 \\
\text { years and } \\
\text { parents }\end{array}$ & $\begin{array}{l}\text { Severity and } \\
\text { duration of } \\
\text { disaster } \\
\text { exposure } \\
\text { and loss, } \\
\text { gender, } \\
\text { parental } \\
\text { depression, } \\
3 \text { cohort of } \\
\text { coping } \\
\text { power } \\
\text { program }\end{array}$ & $\begin{array}{l}\text { Pre-tornado, } \\
\text { levels of } \\
\text { anxiety }\end{array}$ & $\begin{array}{l}\text { Pre disaster } \\
\text { (T1) } \\
6 \text { months } \\
\text { post disaster } \\
\text { (T2) } \\
12 \text { months } \\
\text { post disaster } \\
\text { (T3) }\end{array}$ & $\begin{array}{l}\text { Child } \\
\text { psychologi } \\
\text { cal } \\
\text { and } \\
\text { behavioral } \\
\text { adjustment } \\
\text { Aggressive } \\
\text { behavior } \\
\text { Internalizi } \\
\text { ng } \\
\text { behavior }\end{array}$ \\
\hline
\end{tabular}




\section{RESILIENCE IN CHILDREN AFTER NATURAL DISASTERS}

\begin{tabular}{|c|c|c|c|c|c|c|c|c|}
\hline $\begin{array}{l}\text { Daugherty, T. } \\
\text { K., \& Taylor, } \\
\text { C. M. } \\
\text { (1991). }\end{array}$ & & & & & & & & \\
\hline $\begin{array}{l}\text { Lowe, S. R., } \\
\text { Godoy, L., } \\
\text { Rhodes, J. E., } \\
\text { \& Carter, A. } \\
\text { S. (2013). }\end{array}$ & $\begin{array}{l}\text { Hurricane } \\
\text { (USA) }\end{array}$ & $\begin{array}{l}\text { Pre-post } \\
\text { and } \\
\text { Longitudin } \\
\text { al }\end{array}$ & $\begin{array}{l}\text { Individual } \\
\text { and } \\
\text { Interpersona } \\
1\end{array}$ & $\begin{array}{l}184 \text { mothers } \\
\text { and } \\
251 \text { children } \\
\text { aged } 5-10\end{array}$ & $\begin{array}{l}\text { Age, sex, } \\
\text { disaster } \\
\text { exposures } \\
\text { and related } \\
\text { experiences, } \\
\text { stressor }\end{array}$ & $\begin{array}{l}\text { Maternal } \\
\text { psychologi } \\
\text { cal distress } \\
\text { School } \\
\text { mobility }\end{array}$ & $\begin{array}{l}12 \text { months } \\
\text { pre disaster } \\
\text { (T1) } \\
12 \text { months } \\
\text { post disaster } \\
\text { (T2) } \\
3 \text { years } \\
\text { post disaster } \\
\text { (T3) }\end{array}$ & $\begin{array}{l}\text { Child } \\
\text { symptoms } \\
\text { (internalizi } \\
\text { ng, } \\
\text { externalizi } \\
\text { ng) }\end{array}$ \\
\hline $\begin{array}{l}\text { Ma, X., Liu, } \\
\text { X., Hu, X., } \\
\text { Qiu, C., } \\
\text { Wang, Y., } \\
\text { Huang, Y., ... } \\
\text { \& Li, T. } \\
\text { (2011). }\end{array}$ & $\begin{array}{l}\text { Earthquake } \\
\text { (China) }\end{array}$ & $\begin{array}{l}\text { Cross- } \\
\text { sectional }\end{array}$ & $\begin{array}{l}\text { Individual } \\
\text { and } \\
\text { Interpersona } \\
1\end{array}$ & $\begin{array}{l}3645 \\
\text { adolescents } \\
\text { aged 12-18 } \\
\text { years }\end{array}$ & $\begin{array}{l}\text { Demographi } \\
\text { c data, } \\
\text { disaster } \\
\text { exposure, } \\
\text { cognitive } \\
\text { status, } \\
\text { social } \\
\text { supports }\end{array}$ & & $\begin{array}{l}6 \text { months } \\
\text { post disaster }\end{array}$ & $\begin{array}{l}\text { PTSD } \\
\text { Post- } \\
\text { trauma } \\
\text { stress } \\
\text { symptoms }\end{array}$ \\
\hline
\end{tabular}




\section{RESILIENCE IN CHILDREN AFTER NATURAL DISASTERS}

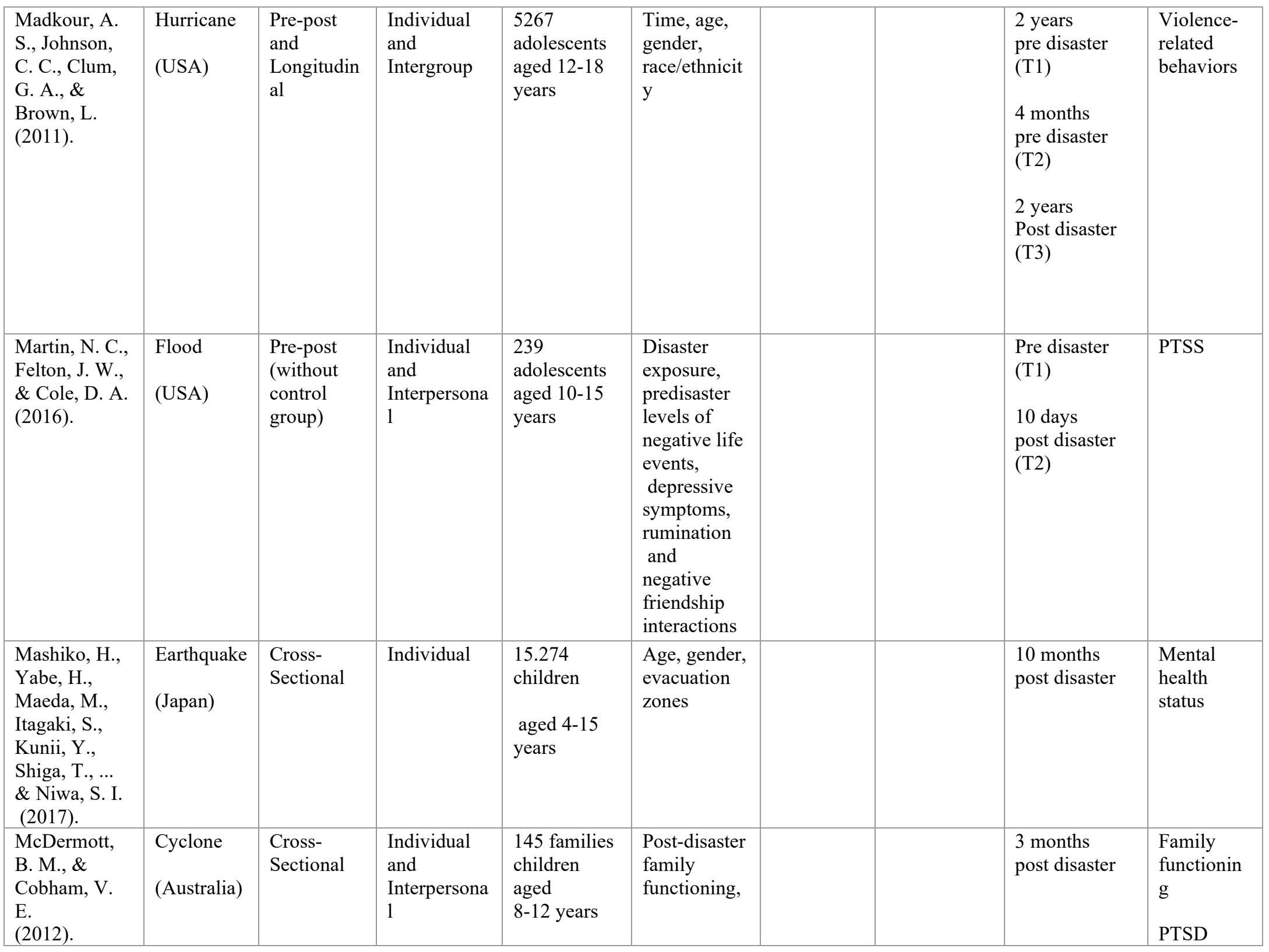




\begin{tabular}{|c|c|c|c|c|c|c|c|}
\hline & & & & & $\begin{array}{l}\text { disaster- } \\
\text { related } \\
\text { variables } \\
\text { threat to self } \\
\text { family } \\
\text { health and/or } \\
\text { dysfunction, } \\
\text { age, gender }\end{array}$ & & \\
\hline $\begin{array}{l}\text { McDermott, } \\
\text { B. M., } \\
\text { Cobham, V. } \\
\text { E., Berry, H., } \\
\text { \& Stallman, } \\
\text { H. M. (2010). }\end{array}$ & $\begin{array}{l}\text { Cyclone } \\
\text { (Australia) }\end{array}$ & $\begin{array}{l}\text { Cross- } \\
\text { Sectional }\end{array}$ & $\begin{array}{l}\text { Individual } \\
\text { and } \\
\text { Interpersona } \\
1\end{array}$ & $\begin{array}{l}568 \text { children } \\
\text { aged } \\
8-15 \text { years }\end{array}$ & $\begin{array}{l}\text { Family } \\
\text { resilience, } \\
\text { event-related } \\
\text { factors, } \\
\text { previous } \\
\text { child mental } \\
\text { illness, } \\
\text { social } \\
\text { connectedne } \\
\text { ss, } \\
\text { disaster } \\
\text { exposure, } \\
\text { age, grade }\end{array}$ & $\begin{array}{l}3 \text { months } \\
\text { post disaster }\end{array}$ & PTSD \\
\hline $\begin{array}{l}\text { McDermott, } \\
\text { B., Berry, H., } \\
\text { \& Cobham, } \\
\text { V. (2012). }\end{array}$ & $\begin{array}{l}\text { Cyclone } \\
\text { (Australia) }\end{array}$ & $\begin{array}{l}\text { Cross- } \\
\text { Sectional }\end{array}$ & $\begin{array}{l}\text { Individual } \\
\text { and } \\
\text { Interpersona } \\
1\end{array}$ & $\begin{array}{l}804 \text { children } \\
\text { aged } 8-13 \\
\text { years }\end{array}$ & $\begin{array}{l}\text { Age, } \\
\text { gender, } \\
\text { school } \\
\text { system, } \\
\text { trauma } \\
\text { exposure, } \\
\text { threat } \\
\text { perception, } \\
\text { new social } \\
\text { connectedne } \\
\text { ss }\end{array}$ & $\begin{array}{l}3 \text { months } \\
\text { post disaster }\end{array}$ & PTSD \\
\hline $\begin{array}{l}\text { McDermott, } \\
\text { B., Cobham, } \\
\text { V., Berry, H., } \\
\text { \& Kim, B. } \\
(2014) .\end{array}$ & $\begin{array}{l}\text { Cyclone } \\
\text { (Australia) }\end{array}$ & $\begin{array}{l}\text { Longitudin } \\
\text { al }\end{array}$ & $\begin{array}{l}\text { Individual } \\
\text { and } \\
\text { Interpersona } \\
1\end{array}$ & $\begin{array}{l}71 \text { children } \\
\text { And } \\
191 \\
\text { adolescents } \\
\text { grades } 5^{\text {th }}- \\
10^{\text {th }}\end{array}$ & $\begin{array}{l}\text { Disaster } \\
\text { exposure, } \\
\text { social } \\
\text { connectedne } \\
\text { ss, } \\
\text { PTSD, age, } \\
\text { time }\end{array}$ & $\begin{array}{l}3 \text { months } \\
\text { post disaster } \\
18 \text { months } \\
\text { Post disaster }\end{array}$ & PTSD \\
\hline
\end{tabular}




\begin{tabular}{|c|c|c|c|c|c|c|c|c|}
\hline $\begin{array}{l}\text { McDonald } \\
\text { KL, Vernberg } \\
\text { EM, Lochman } \\
\text { JE, et al. } \\
(2019)\end{array}$ & $\begin{array}{l}\text { Tornado } \\
\text { (USA) }\end{array}$ & $\begin{array}{l}\text { Longitudin } \\
\text { al }\end{array}$ & $\begin{array}{l}\text { Individual } \\
\text { Interpersona } \\
1\end{array}$ & $\begin{array}{l}346 \text { 4th-6th- } \\
\text { graders and } \\
\text { their } \\
\text { caregivers }\end{array}$ & $\begin{array}{l}\text { Predisaster } \\
\text { individual } \\
\text { and family } \\
\text { characteristi } \\
\text { cs } \\
\text { Disaster } \\
\text { exposure }\end{array}$ & $\begin{array}{l}\text { Positive } \\
\text { parenting } \\
\text { and } \\
\text { pretornado } \\
\text { caregiver } \\
\text { trauma } \\
\text { exposure }\end{array}$ & $\begin{array}{l}4 \text { years after } \\
\text { tornado. }\end{array}$ & PTSS \\
\hline $\begin{array}{l}\text { McFarlane, } \\
\text { A.C. } \\
(1987)\end{array}$ & $\begin{array}{l}\text { Bushfires } \\
\text { (South } \\
\text { Australia) }\end{array}$ & $\begin{array}{l}\text { Longitudin } \\
\text { al }\end{array}$ & $\begin{array}{l}\text { Individual } \\
\text { and } \\
\text { Interpersona } \\
1\end{array}$ & $\begin{array}{l}150 \text { children } \\
\text { (T1-T2) } \\
101 \text { children } \\
\text { (T3) } \\
\text { and parents } \\
\text { (T2-T3) } \\
\text { aged 6-13 } \\
\text { years }\end{array}$ & $\begin{array}{l}\text { Disaster } \\
\text { exposure }\end{array}$ & $\begin{array}{l}\text { Family } \\
\text { functions: } \\
\text { - irritable } \\
\text { distress } \\
\text { - } \\
\text { involvement } \\
\text { Overprotecti } \\
\text { on }\end{array}$ & $\begin{array}{l}2 \text { months } \\
\text { post disaster } \\
8 \text { months } \\
\text { post disaster } \\
\text { (T2) } \\
26 \text { months } \\
\text { post disaster } \\
\text { (T3) }\end{array}$ & $\begin{array}{l}\text { Emotional } \\
\text { and } \\
\text { behavioura } \\
1 \text { problems } \\
\text { Children } \\
\text { disorders }\end{array}$ \\
\hline $\begin{array}{l}\text { McLaughlin, } \\
\text { K. A., } \\
\text { Fairbank, J. } \\
\text { A., Gruber, } \\
\text { M. J., Jones, } \\
\text { R. T., } \\
\text { Osofsky, J. } \\
\text { D., } \\
\text { Pfefferbaum, } \\
\text { B., ... \& } \\
\text { Kessler, R. C. } \\
\text { (2010). }\end{array}$ & $\begin{array}{l}\text { Hurricane } \\
\text { (USA) }\end{array}$ & $\begin{array}{l}\text { Longitudin } \\
\text { al }\end{array}$ & Individual & $\begin{array}{l}576 \text { children } \\
\text { and } \\
\text { adolescents } \\
\text { aged } 4-17 \\
\text { years }\end{array}$ & $\begin{array}{l}\text { Stressors } \\
\text { experienced } \\
\text { in the } \\
\text { hurricane, } \\
\text { ongoing } \\
\text { stressors }\end{array}$ & & $\begin{array}{l}5-7 \text { months } \\
\text { post disaster } \\
\text { (T1) } \\
7 \text { to } 10 \text { months } \\
\text { post disaster } \\
\text { (T2) } \\
15-19 \text { months } \\
\text { post disaster } \\
\text { (T3) }\end{array}$ & $\begin{array}{l}\text { Emotional } \\
\text { functionin } \\
\mathrm{g} \text { in } \\
\text { children } \\
\text { Emotional } \\
\text { disturbanc } \\
\mathrm{e}\end{array}$ \\
\hline $\begin{array}{l}\text { McLaughlin, } \\
\text { K. A., } \\
\text { Fairbank, J. } \\
\text { A., Gruber, } \\
\text { M. J., Jones, } \\
\text { R. T., } \\
\text { Lakoma, M. } \\
\text { D., } \\
\text { Pfefferbaum, } \\
\text { B., ... \& } \\
\text { Kessler, R. C. } \\
\text { (2009). }\end{array}$ & $\begin{array}{l}\text { Hurricane } \\
\text { (USA) }\end{array}$ & $\begin{array}{l}\text { Cross- } \\
\text { Sectional }\end{array}$ & $\begin{array}{l}\text { Individual } \\
\text { and } \\
\text { Interpersona } \\
1\end{array}$ & $\begin{array}{l}797 \text { children } \\
\text { aged } 4-17 \\
\text { years }\end{array}$ & $\begin{array}{l}\text { Hurricane- } \\
\text { related } \\
\text { stressors, } \\
\text { demographic } \\
\text { s, family } \\
\text { factors }\end{array}$ & & $\begin{array}{l}18-27 \text { months } \\
\text { post disaster }\end{array}$ & $\begin{array}{l}\text { Emotional } \\
\text { disturbanc } \\
\mathrm{e}\end{array}$ \\
\hline
\end{tabular}




\section{RESILIENCE IN CHILDREN AFTER NATURAL DISASTERS}

\begin{tabular}{|c|c|c|c|c|c|c|c|c|}
\hline $\begin{array}{l}\text { McLean, M. } \\
\text { A., Cobham, } \\
\text { V. E., } \\
\text { Simcock, G., } \\
\text { Elgbeili, G., } \\
\text { Kildea, S., \& } \\
\text { King, S. } \\
\text { (2018) }\end{array}$ & $\begin{array}{l}\text { Flood } \\
\text { (USA) }\end{array}$ & $\begin{array}{l}\text { Cross- } \\
\text { sectional }\end{array}$ & $\begin{array}{l}\text { Individual } \\
\text { Interpersona } \\
1\end{array}$ & $\begin{array}{l}230 \text { mothers } \\
\text { and } 118 \\
\text { children } 4 \\
\text { years old }\end{array}$ & $\begin{array}{l}\text { prenatal } \\
\text { maternal } \\
\text { stress } \\
\text { maternal } \\
\text { socioeconom } \\
\text { ic status, } \\
\text { education, } \\
\text { income, and } \\
\text { marital } \\
\text { status. } \\
\text { Major life } \\
\text { events. } \\
\text { Infant birth } \\
\text { weight and } \\
\text { gestational } \\
\text { age }\end{array}$ & $\begin{array}{l}\text { Timing of } \\
\text { flood } \\
\text { exposure } \\
\text { during } \\
\text { pregnancy } \\
\text { and child sex }\end{array}$ & $\begin{array}{l}12 \text { months } \\
\text { postflood }\end{array}$ & $\begin{array}{l}\text { Children } \\
\text { Anxiety }\end{array}$ \\
\hline $\begin{array}{l}\text { Meyer, A., } \\
\text { Danielson, C. } \\
\text { K., Danzig, A. } \\
\text { P., Bhatia, V., } \\
\text { Black, S. R., } \\
\text { Bromet, E., ... } \\
\text { \& Klein, D. } \\
\text { N. (2017). }\end{array}$ & $\begin{array}{l}\text { Hurricane } \\
\text { (USA) }\end{array}$ & $\begin{array}{l}\text { Pre-post } \\
\text { and } \\
\text { Longitudin } \\
\text { al }\end{array}$ & Individual & $\begin{array}{l}223 \text { children, } \\
\text { aged } \\
\text { between } \\
3.00-4.09 \\
\text { years and } \\
\text { theirs } \\
\text { mothers }\end{array}$ & $\begin{array}{l}\text { Age, } \\
\text { temperament } \\
\text { al fear, } \\
\text { biomarker of } \\
\text { risk for } \\
\text { anxiety, } \\
\text { fearfulness, } \\
\text { hurricane } \\
\text { stressors, } \\
\text { ern (error- } \\
\text { related } \\
\text { negativity) }\end{array}$ & & $\begin{array}{l}3 \text { years of age, } \\
6 \text { years of age, } \\
\text { and } 9 \text { years of } \\
\text { age } \\
\text { (pre disaster } \\
\text { assessment) } \\
6 \text { weeks } \\
\text { post disaster }\end{array}$ & $\begin{array}{l}\text { Internalizi } \\
\text { ng } \\
\text { symptoms }\end{array}$ \\
\hline $\begin{array}{l}\text { Mikolajewski, } \\
\text { A. J., \& } \\
\text { Scheeringa, } \\
\text { M. S. (2018) }\end{array}$ & $\begin{array}{l}\text { Hurricane } \\
\text { (USA) }\end{array}$ & $\begin{array}{l}\text { Cross- } \\
\text { sectional }\end{array}$ & Individual & $\begin{array}{l}36 \text { children } \\
3-6 \text { years } \\
\text { old }\end{array}$ & $\begin{array}{l}\text { Age } \\
\text { Respiratory } \\
\text { Sinus } \\
\text { Arrhythmia }\end{array}$ & & $\begin{array}{l}19 \text { to } 681 \text { days } \\
\text { before disaster } \\
8 \text { months to } 3 \\
\text { yars after } \\
\text { disaster }\end{array}$ & PTSD \\
\hline $\begin{array}{l}\text { Mikyung, J., } \\
\text { Se-hwa, L. \& } \\
\text { Lee-jin, K. } \\
(2020)\end{array}$ & $\begin{array}{l}\text { Earthquake } \\
\text { (Nepal) }\end{array}$ & $\begin{array}{l}\text { Cross- } \\
\text { sectional }\end{array}$ & $\begin{array}{l}\text { Individual } \\
\text { Intepersonal }\end{array}$ & $\begin{array}{l}200 \text { pairs } \\
\text { parents and } \\
\text { children } \\
6-17 \text { years } \\
\text { old }\end{array}$ & $\begin{array}{l}\text { PTSD } \\
\text { and } \\
\text { internalizing } \\
\text { and } \\
\text { externalizing } \\
\text { problems in } \\
\text { parents }\end{array}$ & & $\begin{array}{l}24 \text { months } \\
\text { after } \\
\text { earthquake }\end{array}$ & $\begin{array}{l}\text { PTSD } \\
\text { internalizin } \\
\mathrm{g} \text { and } \\
\text { externalizi } \\
\text { ng } \\
\text { problems } \\
\text { in children }\end{array}$ \\
\hline
\end{tabular}




\begin{tabular}{|c|c|c|c|c|c|c|c|}
\hline & & & & & $\begin{array}{l}\text { Education } \\
\text { Occupation }\end{array}$ & & \\
\hline $\begin{array}{l}\text { Miller, P. A., } \\
\text { Roberts, N. } \\
\text { A., Zamora, } \\
\text { A. D., Weber, } \\
\text { D. J., } \\
\text { Burleson, M. } \\
\text { H., Robles, } \\
\text { E., \& Tinsley, } \\
\text { B. J. (2012). }\end{array}$ & $\begin{array}{l}\text { Wildfire } \\
\text { And } \\
\text { Tornado } \\
\text { (USA) }\end{array}$ & $\begin{array}{l}\text { Cross- } \\
\text { Sectional }\end{array}$ & $\begin{array}{l}\text { Individual } \\
\text { and } \\
\text { Interpersona } \\
1\end{array}$ & $\begin{array}{l}24 \text { parents } \\
\text { and } 44 \\
\text { children } \\
\text { aged 5-17 } \\
\text { years } \\
32 \text { parents } \\
\text { and } \\
63 \text { children } \\
\text { aged from } 6 \\
\text { weeks to } 18 \\
\text { years }\end{array}$ & $\begin{array}{l}\text { Coping } \\
\text { activities, } \\
\text { prior and } \\
\text { current } \\
\text { disaster } \\
\text { exposure } \\
\text { levels, } \\
\text { family } \\
\text { coping, } \\
\text { social } \\
\text { support }\end{array}$ & $\begin{array}{l}\text { Within } 4 \text { days } \\
\text { of each } \\
\text { disaster }\end{array}$ & $\begin{array}{l}\text { Psychologi } \\
\text { cal impact } \\
\text { of disasters } \\
\text { on children }\end{array}$ \\
\hline $\begin{array}{l}\text { Milne, G. } \\
\text { (1977). }\end{array}$ & $\begin{array}{l}\text { Cyclone } \\
\text { (Australia) }\end{array}$ & $\begin{array}{l}\text { Cross- } \\
\text { Sectional }\end{array}$ & Individual & $\begin{array}{l}649 \text { children } \\
\text { aged 5-16 } \\
\text { years }\end{array}$ & $\begin{array}{l}\text { Evacuated or } \\
\text { not, } \\
\text { age, } \\
\text { gender }\end{array}$ & & $\begin{array}{l}\begin{array}{l}\text { School } \\
\text { problem }\end{array} \\
\text { Fearful, } \\
\text { regressive, } \\
\text { and } \\
\text { aggressive } \\
\text { behaviour } \\
\text { Injuries, } \\
\text { diseases } \\
\text { and } \\
\text { infections } \\
\text { Emotional } \\
\text { and } \\
\text { physical } \\
\text { disorders }\end{array}$ \\
\hline $\begin{array}{l}\text { Moore, K. W., } \\
\& \text { Varela, R. } \\
\text { E. (2010). }\end{array}$ & $\begin{array}{l}\text { Hurricane } \\
\text { (USA) }\end{array}$ & $\begin{array}{l}\text { Cross- } \\
\text { Sectional }\end{array}$ & $\begin{array}{l}\text { Individual } \\
\text { and } \\
\text { Interpersona } \\
1\end{array}$ & $\begin{array}{l}156 \text { children } \\
\text { aged } 9-14 \\
\text { years }\end{array}$ & $\begin{array}{l}\text { Gender, } \\
\text { disaster } \\
\text { exposure, } \\
\text { parent } \\
\text { support, }\end{array}$ & $\begin{array}{l}33 \text { months } \\
\text { post disaster }\end{array}$ & PTSS \\
\hline
\end{tabular}




\section{RESILIENCE IN CHILDREN AFTER NATURAL DISASTERS}

\begin{tabular}{|c|c|c|c|c|c|c|c|c|c|}
\hline & & & & & $\begin{array}{l}\text { classmate } \\
\text { support, } \\
\text { teacher } \\
\text { support } \\
\text { friend } \\
\text { support } \\
\text { immediate } \\
\text { loss } \\
\text { disruption } \\
\text { continued } \\
\text { loss } \\
\text { and } \\
\text { disruption } \\
\text { negative } \\
\text { events }\end{array}$ & & & & \\
\hline $\begin{array}{l}\text { Mordeno, I. } \\
\text { G., Galela, D. } \\
\text { S., Nalipay, } \\
\text { M. J. N., \& } \\
\text { Cue, M. P. } \\
\text { (2018) }\end{array}$ & $\begin{array}{l}\text { Typhoon } \\
\text { (Philippine } \\
\text { ) }\end{array}$ & $\begin{array}{l}\text { Cross- } \\
\text { sectional }\end{array}$ & Individual & $\begin{array}{l}225 \text { children } \\
9-17 \text { years } \\
\text { old }\end{array}$ & $\begin{array}{l}\text { Centrality } \\
\text { of Event }\end{array}$ & $\begin{array}{l}\text { Sensory- } \\
\text { based } \\
\text { trauma } \\
\text { memory } \\
\text { quality }\end{array}$ & & & $\begin{array}{l}\text { Acute } \\
\text { Stress } \\
\text { Disorder } \\
\text { Depression }\end{array}$ \\
\hline $\begin{array}{l}\text { Moss, K. M., } \\
\text { Simcock, G., } \\
\text { Cobham, V., } \\
\text { Kildea, S., } \\
\text { Elgbeili, G., } \\
\text { Laplante, D. } \\
\text { P., \& King, S. } \\
\text { (2017). }\end{array}$ & $\begin{array}{l}\text { Flood } \\
\text { (Australia) }\end{array}$ & $\begin{array}{l}\text { Longitudin } \\
\text { al }\end{array}$ & $\begin{array}{l}\text { Individual } \\
\text { and } \\
\text { Interpersona } \\
1\end{array}$ & $\begin{array}{l}145 \\
\text { mothers }\end{array}$ & $\begin{array}{l}\text { Prenatal } \\
\text { maternal } \\
\text { stress, } \\
\text { maternal } \\
\text { severity of } \\
\text { flood } \\
\text { exposure } \\
\text { subjective } \\
\text { stress } \\
\text { reactions } \\
\text { cognitive } \\
\text { appraisal }\end{array}$ & & $\begin{array}{l}\text { Timing } \\
\text { of exposure }\end{array}$ & $\begin{array}{l}12 \text { months } \\
\text { post disaster } \\
16 \text { months } \\
\text { postpartum }\end{array}$ & $\begin{array}{l}\text { Child } \\
\text { cognitive } \\
\text { and motor } \\
\text { developme } \\
\text { nt }\end{array}$ \\
\hline $\begin{array}{l}\text { Mouchenik, } \\
\text { Y., Marty- } \\
\text { Chevreuil, A., } \\
\text { Marquer, C., } \\
\text { Joseph, N. E., } \\
\text { Ducasse, J. } \\
\text { W., Ryswick, }\end{array}$ & $\begin{array}{l}\text { Earthquake } \\
\text { (Haiti) }\end{array}$ & $\begin{array}{l}\text { Cross- } \\
\text { Sectional }\end{array}$ & Individual & $\begin{array}{l}166 \text { children } \\
\text { aged 3-6 } \\
\text { years }\end{array}$ & $\begin{array}{l}\text { Demographi } \\
\text { c aspects, } \\
\text { gender, } \\
\text { age, trauma } \\
\text { exposure }\end{array}$ & & & $\begin{array}{l}11 \text { months } \\
\text { post disaster }\end{array}$ & $\begin{array}{l}\text { Psychologi } \\
\text { cal } \\
\text { disturbanc } \\
\text { e }\end{array}$ \\
\hline
\end{tabular}




\section{RESILIENCE IN CHILDREN AFTER NATURAL DISASTERS}

\begin{tabular}{|c|c|c|c|c|c|c|c|c|}
\hline $\begin{array}{l}\text { C., ... \& } \\
\text { Baubet, T. } \\
(2014) .\end{array}$ & & & & & & & & \\
\hline $\begin{array}{l}\text { Mumper, E. } \\
\text { E., Dyson, M. } \\
\text { W., Finsaas, } \\
\text { M. C., Olino, } \\
\text { T. M., \& } \\
\text { Klein, D. N. } \\
(2019)\end{array}$ & $\begin{array}{l}\text { Hurricane } \\
\text { (USA) }\end{array}$ & $\begin{array}{l}\text { Longitudin } \\
\text { al }\end{array}$ & Invidual & $\begin{array}{l}392 \text { children } \\
3 \text { years-old }\end{array}$ & $\begin{array}{l}\text { Sex } \\
\text { Age } \\
\text { behavioral } \\
\text { inhibition }\end{array}$ & $\begin{array}{l}\text { Life stress } \\
\text { events and } \\
\text { natural } \\
\text { disaster } \\
\text { exposure }\end{array}$ & $\begin{array}{l}7 \text { year before } \\
\text { hurricane, six } \\
\text { weeks after } \\
\text { hurricane and } \\
2 \text { years after } \\
\text { the hurricane }\end{array}$ & Anxiety \\
\hline $\begin{array}{l}\text { Muris, P., } \\
\text { Meesters, C., } \\
\text { Merckelbach, } \\
\text { H., } \\
\text { Verschuren, } \\
\text { M., Geebelen, } \\
\text { E., \& Aleva, } \\
\text { E. (2002). }\end{array}$ & $\begin{array}{l}\text { Hurricane } \\
\text { (Antilles) }\end{array}$ & $\begin{array}{l}\text { Experiment } \\
\text { al (with } \\
\text { control } \\
\text { group) }\end{array}$ & Individual & $\begin{array}{l}161 \\
\text { Antillean } \\
\text { children } \\
\text { disaster } \\
\text { exposure } \\
185 \text { Belgian } \\
\text { control } \\
\text { group } \\
\text { aged 8-11 } \\
\text { years }\end{array}$ & $\begin{array}{l}\text { Age, } \\
\text { gender, } \\
\text { different } \\
\text { forms } \\
\text { of fear } \\
\text { related and } \\
\text { not related to } \\
\text { disasters }\end{array}$ & & & $\begin{array}{l}\text { Fear of } \\
\text { storms }\end{array}$ \\
\hline $\begin{array}{l}\text { Najarian, L. } \\
\text { M., Goenjian, } \\
\text { A. K., } \\
\text { Pelcovttz, D., } \\
\text { Mandel, F., \& } \\
\text { Najarian, B. } \\
\text { (1996). }\end{array}$ & $\begin{array}{l}\text { Earthquake } \\
\text { (Armenia) }\end{array}$ & $\begin{array}{l}\text { Cross- } \\
\text { Sectional }\end{array}$ & Individual & $\begin{array}{l}74 \text { children } \\
\text { aged 11-13 } \\
\text { years }\end{array}$ & $\begin{array}{l}\text { Trauma } \\
\text { exposure, } \\
\text { relocation }\end{array}$ & & $\begin{array}{l}2.5 \text { years } \\
\text { post disaster }\end{array}$ & $\begin{array}{l}\text { PTSD, } \\
\text { Depression } \\
\text { behavioral } \\
\text { difficulties }\end{array}$ \\
\hline $\begin{array}{l}\text { Navarro, J., } \\
\text { Pulido, R., } \\
\text { Berger, C., } \\
\text { Arteaga, M., } \\
\text { Osofsky, H. } \\
\text { J., Martinez, } \\
\text { M., ... \& } \\
\text { Hansel, T. C. } \\
\text { (2016). }\end{array}$ & $\begin{array}{l}\text { Earthquake } \\
\text { Tsunami } \\
\text { Hurricane } \\
\text { (USA) }\end{array}$ & $\begin{array}{l}\text { Cross- } \\
\text { Sectional }\end{array}$ & Individual & $\begin{array}{l}827 \text { children } \\
\text { and } \\
\text { adolescents } \\
\text { aged } 8-17 \\
\text { years }\end{array}$ & $\begin{array}{l}\text { Type of } \\
\text { disaster }\end{array}$ & & $\begin{array}{l}4 \text { months } \\
\text { post disaster }\end{array}$ & $\begin{array}{l}\text { Depression } \\
\text { PTS }\end{array}$ \\
\hline
\end{tabular}




\section{RESILIENCE IN CHILDREN AFTER NATURAL DISASTERS}

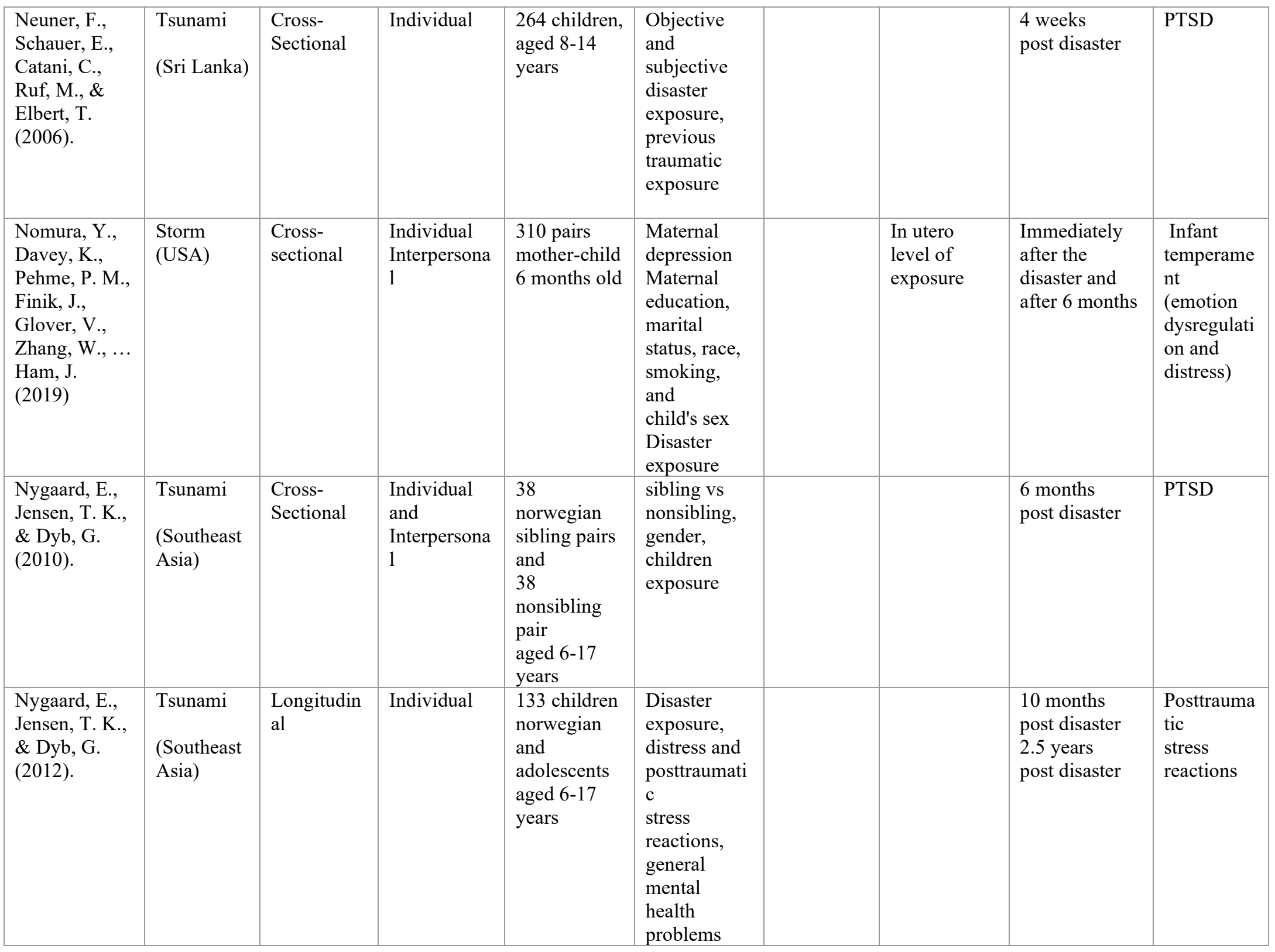




\section{RESILIENCE IN CHILDREN AFTER NATURAL DISASTERS}

\begin{tabular}{|c|c|c|c|c|c|c|c|c|}
\hline $\begin{array}{l}\text { Ortiz, C. D., } \\
\text { Silverman, W. } \\
\text { K., Jaccard, } \\
\text { J., \& La } \\
\text { Greca, A. M. } \\
(2011) .\end{array}$ & $\begin{array}{l}\text { Hurricane } \\
\text { (USA) }\end{array}$ & $\begin{array}{l}\text { Experiment } \\
\text { al (with } \\
\text { control } \\
\text { group) }\end{array}$ & $\begin{array}{l}\text { Individual } \\
\text { and } \\
\text { Interpersona } \\
1\end{array}$ & $\begin{array}{l}248 \text { children } \\
\text { from a } \\
\text { hurricane } \\
\text { prone region } \\
63 \text { control } \\
\text { group } \\
\text { (neutral } \\
\text { film) } \\
185 \\
\text { exposure to } \\
\text { disaster } \\
\text { media } \\
\text { aged } 7-12 \\
\text { years }\end{array}$ & $\begin{array}{l}\text { Life event, } \\
\text { anxiety } \\
\text { depression } \\
\text { social } \\
\text { support and } \\
\text { use of } \\
\text { coping } \\
\text { strategies }\end{array}$ & $\begin{array}{l}\text { Grade } \\
\text { Sex }\end{array}$ & & $\begin{array}{l}\text { State } \\
\text { anxiety }\end{array}$ \\
\hline $\begin{array}{l}\text { Osofsky, H. } \\
\text { J., Osofsky, J. } \\
\text { D., } \\
\text { Kronenberg, } \\
\text { M., Brennan, } \\
\text { A., \& Hansel, } \\
\text { T. C. (2009). }\end{array}$ & $\begin{array}{l}\text { Hurricane } \\
\text { (USA) }\end{array}$ & $\begin{array}{l}\text { Cross- } \\
\text { sectional }\end{array}$ & Individual & $\begin{array}{l}7258 \\
\text { children } \\
\text { aged } 7-19\end{array}$ & $\begin{array}{l}\text { Disaster } \\
\text { exposure, } \\
\text { demographic } \\
\text { variables }\end{array}$ & & $\begin{array}{l}2 \text { years } \\
\text { post disaster }\end{array}$ & PTSS \\
\hline $\begin{array}{l}\text { Osofsky, J. } \\
\text { D., Osofsky, } \\
\text { H. J., Weems, } \\
\text { C. F., Hansel, } \\
\text { T. C., \& King, } \\
\text { L. S. (2014). }\end{array}$ & $\begin{array}{l}\text { Gulf Oil } \\
\text { Spill and } \\
\text { Hurricane } \\
\text { (USA) }\end{array}$ & $\begin{array}{l}\text { Pre - post } \\
\text { (without } \\
\text { control } \\
\text { group) }\end{array}$ & Individual & $\begin{array}{l}1730 \\
\text { children and } \\
\text { adolescents } \\
\text { aged } 3-18 \\
\text { years }\end{array}$ & $\begin{array}{l}\text { Level of oil } \\
\text { spill stress, } \\
\text { preexisting } \\
\text { PTSD } \\
\text { symptoms, } \\
\text { previous } \\
\text { hurricane } \\
\text { exposure, } \\
\text { gender } \\
\text { minority } \\
\text { status }\end{array}$ & & $\begin{array}{l}1 \text { year } \\
\text { Pre-disaster } \\
1 \text { year } \\
\text { post disaster }\end{array}$ & PTSD \\
\hline $\begin{array}{l}\text { Osofsky, J.D., } \\
\text { Kronenberg, } \\
\text { M., Bocknek, } \\
\text { E., \& Hansel, } \\
\text { T. C. (2015). }\end{array}$ & $\begin{array}{l}\text { Hurricane } \\
\text { (USA) }\end{array}$ & $\begin{array}{l}\text { Longitudin } \\
\text { al }\end{array}$ & $\begin{array}{l}\text { Individual } \\
\text { and } \\
\text { Interpersona } \\
1\end{array}$ & $\begin{array}{l}914 \text { children } \\
\text { aged } 3-5 \\
\text { years }\end{array}$ & $\begin{array}{l}\text { Attachment, } \\
\text { caregiver } \\
\text { disruption, } \\
\text { nonhuman } \\
\text { losses, } \\
\text { disaster } \\
\text { exposure }\end{array}$ & & $\begin{array}{l}\text { During the } \\
\text { first year post } \\
\text { disaster } \\
\text { (T1) } \\
\text { After 1 year } \\
\text { (T2) }\end{array}$ & $\begin{array}{l}\text { Children's } \\
\text { long-term } \\
\text { post- } \\
\text { hurricane } \\
\text { psychologi } \\
\text { cal } \\
\text { outcome }\end{array}$ \\
\hline
\end{tabular}




\begin{tabular}{|c|c|c|c|c|c|c|c|c|}
\hline & & & & & & & $\begin{array}{l}\text { After } 2 \text { years } \\
\text { (T3) } \\
\text { After } 3 \text { years } \\
\text { (T4) }\end{array}$ & \\
\hline $\begin{array}{l}\text { Pina, A. A., } \\
\text { Villalta, I. K., } \\
\text { Ortiz, C. D., } \\
\text { Gottschall, A. } \\
\text { C., Costa, N. } \\
\text { M., \& } \\
\text { Weems, C. F. } \\
\text { (2008). }\end{array}$ & $\begin{array}{l}\text { Hurricane } \\
\text { (USA) }\end{array}$ & $\begin{array}{l}\text { Pre-post } \\
\text { (without } \\
\text { control } \\
\text { group) }\end{array}$ & Individual & $\begin{array}{l}46 \text { youth } \\
(\mathrm{M}=11.43)\end{array}$ & $\begin{array}{l}\text { Disaster } \\
\text { exposure, } \\
\text { social } \\
\text { support, } \\
\text { discriminatio } \\
\mathrm{n} \text { and coping }\end{array}$ & & $\begin{array}{l}\text { Pre-disaster } \\
\text { 6-7 months } \\
\text { post disaster }\end{array}$ & $\begin{array}{l}\text { PTSD, } \\
\text { anxiety, } \\
\text { and } \\
\text { depression }\end{array}$ \\
\hline $\begin{array}{l}\text { Piotrowski, } \\
\text { C., \& } \\
\text { Dunham, F. } \\
\text { Y. } \\
\text { (1983). }\end{array}$ & $\begin{array}{l}\text { Hurricane } \\
\text { (USA) }\end{array}$ & $\begin{array}{l}\text { Experiment } \\
\text { al } \\
\text { (withour } \\
\text { control } \\
\text { group) }\end{array}$ & Individual & $\begin{array}{l}269 \text { Children } \\
5^{\text {th }} \text { grade } \\
\text { from city hit } \\
\text { by the } \\
\text { hurricane } \\
194 \text { in the } \\
\text { city not hit }\end{array}$ & $\begin{array}{l}\text { Disaster } \\
\text { exposure }\end{array}$ & $\begin{array}{l}\text { Locus of } \\
\text { control }\end{array}$ & $\begin{array}{l}5-8 \text { months } \\
\text { post disaster }\end{array}$ & $\begin{array}{l}\text { Perception } \\
\text { of the } \\
\text { hurricane } \\
\text { concept } \\
\text { "hurricane } \\
\text { " }\end{array}$ \\
\hline $\begin{array}{l}\text { Ponnamperum } \\
\text { a, T., \& } \\
\text { Nicolson, N. } \\
\text { A. } \\
(2016) .\end{array}$ & $\begin{array}{l}\text { Tsunami } \\
\text { (Sri Lanka) }\end{array}$ & $\begin{array}{l}\text { Cross- } \\
\text { Sectional }\end{array}$ & $\begin{array}{l}\text { Individual } \\
\text { and } \\
\text { Interpersona } \\
1\end{array}$ & $\begin{array}{l}414 \\
\text { adolescent } \\
\text { aged 12-16 } \\
\text { years }\end{array}$ & $\begin{array}{l}\text { Negative } \\
\text { appraisals, } \\
\text { lifetime } \\
\text { traumatic } \\
\text { events, } \\
\text { ongoing } \\
\text { adversity, } \\
\text { social } \\
\text { support }\end{array}$ & & $\begin{array}{l}3 \text { years after } \\
\text { the tsunami }\end{array}$ & $\begin{array}{l}\text { PTSS, } \\
\text { internalizin } \\
\text { g } \\
\text { Symptoms } \\
\text { Negative } \\
\text { appraisal } \\
\text { score }\end{array}$ \\
\hline $\begin{array}{l}\text { Prinstein, M. } \\
\text { J., La Greca, } \\
\text { A. M., } \\
\text { Vernberg, E. } \\
\text { M., \& } \\
\text { Silverman, W. } \\
\text { K. } \\
\text { (1996). }\end{array}$ & $\begin{array}{l}\text { Hurricane } \\
\text { (USA) }\end{array}$ & $\begin{array}{l}\text { Cross- } \\
\text { Sectional }\end{array}$ & $\begin{array}{l}\text { Individual } \\
\text { and } \\
\text { Interpersona } \\
1\end{array}$ & $\begin{array}{l}506 \text { children } \\
\text { in the } 3^{\text {rd }}, 4^{\text {th }} \\
\text { and } 5^{\text {th }} \\
\text { grades of } \\
\text { elementary } \\
\text { schools }\end{array}$ & $\begin{array}{l}\text { Children's } \\
\text { social } \\
\text { support, } \\
\text { coping } \\
\text { emotional } \\
\text { processing } \\
\text { and } \\
\text { distraction, } \\
\text { coping }\end{array}$ & & $\begin{array}{l}7 \text { months after } \\
\text { hurricane } \\
\text { Andrew }\end{array}$ & PTSD \\
\hline
\end{tabular}




\section{RESILIENCE IN CHILDREN AFTER NATURAL DISASTERS}

\begin{tabular}{|c|c|c|c|c|c|c|c|c|c|}
\hline $\begin{array}{l}\text { Proctor, L. J., } \\
\text { Fauchier, A., } \\
\text { Oliver, P. H., } \\
\text { Ramos, M. C., } \\
\text { Rios, M. A., } \\
\text { \& Margolin, } \\
\text { G. (2007). }\end{array}$ & $\begin{array}{l}\text { Earthquake } \\
\text { (USA) }\end{array}$ & $\begin{array}{l}\text { Pre-post } \\
\text { (without } \\
\text { control } \\
\text { group) }\end{array}$ & $\begin{array}{l}\text { Individual } \\
\text { and } \\
\text { Interpersona } \\
1\end{array}$ & $\begin{array}{l}117 \text { two- } \\
\text { parent } \\
\text { exposed } \\
\text { families } \\
\text { Children } \\
\text { aged } \\
\text { 4-5 years }\end{array}$ & $\begin{array}{l}\text { Predisaster } \\
\text { parenting } \\
\text { behaviors } \\
\text { and } \\
\text { postdisaster, } \\
\text { parental } \\
\text { stress, } \\
\text { earthquake } \\
\text { impact }\end{array}$ & $\begin{array}{l}\text { Post } \\
\text { parental } \\
\text { stress }\end{array}$ & $\begin{array}{l}\text { Pre- } \\
\text { earthquake } \\
\text { parental } \\
\text { behaviors }\end{array}$ & $\begin{array}{l}8 \text { months after } \\
\text { the earthquake }\end{array}$ & $\begin{array}{l}\text { Parental } \\
\text { stress and } \\
\text { children's } \\
\text { distress }\end{array}$ \\
\hline $\begin{array}{l}\text { Pullins, L. G., } \\
\text { McCammon, } \\
\text { S. L., } \\
\text { Lamson, A. } \\
\text { S., Wuensch, } \\
\text { K. L., \& } \\
\text { Mega, L. } \\
\text { (2005). }\end{array}$ & $\begin{array}{l}\text { Hurricane } \\
\text { and } \\
\text { Flood } \\
\text { (USA) }\end{array}$ & $\begin{array}{l}\text { Cross- } \\
\text { Sectional }\end{array}$ & Individual & $\begin{array}{l}612 \text { children } \\
\text { aged } 5-19 \\
\text { years } \\
248 \text { children } \\
\text { and } 86 \\
\text { parents } \\
\text { participated } \\
\text { in the } \\
\text { evaluation } \\
\text { phase }\end{array}$ & $\begin{array}{l}\text { Grade, } \\
\text { severity of } \\
\text { exposure, } \\
\text { race, gender }\end{array}$ & & & & $\begin{array}{l}\text { Adjustmen } \\
\text { t problems } \\
\text { in children }\end{array}$ \\
\hline $\begin{array}{l}\text { Pynoos, R. S., } \\
\text { Goenjian, A., } \\
\text { Tashjian, M., } \\
\text { Karakashian, } \\
\text { M., } \\
\text { Manjikian, R., } \\
\text { Manoukian, } \\
\text { G., ... \& } \\
\text { Fairbanks, L. } \\
\text { A. (1993). }\end{array}$ & $\begin{array}{l}\text { Earthquake } \\
\text { (Armenia) }\end{array}$ & $\begin{array}{l}\text { Cross- } \\
\text { Sectional }\end{array}$ & Individual & $\begin{array}{l}231 \text { children } \\
\text { aged } 8-16 \\
\text { years }\end{array}$ & $\begin{array}{l}\text { Gender, } \\
\text { proximity to } \\
\text { the } \\
\text { epicentre, } \\
\text { severity of } \\
\text { posttraumati } \\
\text { c stress } \\
\text { reaction }\end{array}$ & & & $\begin{array}{l}18 \text { months } \\
\text { post disaster }\end{array}$ & PTSD \\
\hline $\begin{array}{l}\text { Raccanello, } \\
\text { D., Burro, R., } \\
\text { \& Hall, R. } \\
(2017) \text {. }\end{array}$ & $\begin{array}{l}\text { Earthquake } \\
\text { (Italy) }\end{array}$ & $\begin{array}{l}\text { Experiment } \\
\text { al (with } \\
\text { control } \\
\text { Group) }\end{array}$ & Individual & $\begin{array}{l}127 \text { children } \\
\text { aged } 7-10 \\
\text { years }\end{array}$ & $\begin{array}{l}\text { Group } \\
\text { (experimenta } \\
1, \text { control), } \\
\text { class level } \\
\left(2^{\text {nd }} \text { to } 5^{\text {th }}\right. \\
\text { graders }), \\
\text { gender }\end{array}$ & & & $\begin{array}{l}2 \text { years } \\
\text { post disaster }\end{array}$ & $\begin{array}{l}\text { Children's } \\
\text { emotional } \\
\text { competenc } \\
\text { e: } \\
\text { Understan } \\
\text { ding, } \\
\text { regulating } \\
\text { and }\end{array}$ \\
\hline
\end{tabular}




\begin{tabular}{|c|c|c|c|c|c|c|c|}
\hline & & & & & & & $\begin{array}{l}\text { expressing } \\
\text { emotions } \\
\text { Knowledg } \\
\text { e of } \\
\text { earthquake } \\
\text { s }\end{array}$ \\
\hline $\begin{array}{l}\text { Roberts, Y. } \\
\text { H., Mitchell, } \\
\text { M. J., } \\
\text { Witman, M., } \\
\text { \& Taffaro, C. } \\
(2010) .\end{array}$ & $\begin{array}{l}\text { Hurricane } \\
\text { (USA) }\end{array}$ & $\begin{array}{l}\text { Pre-post } \\
\text { (without } \\
\text { control } \\
\text { group) } \\
\text { and } \\
\text { longitudina } \\
1\end{array}$ & Individual & $\begin{array}{l}43 \text { children } \\
\text { and } \\
\text { adolescent } \\
\text { aged } 11-18 \\
\text { years }\end{array}$ & $\begin{array}{l}\text { Disaster- } \\
\text { related } \\
\text { variables, } \\
\text { demographic } \\
\text { information }\end{array}$ & $\begin{array}{l}\text { Before the } \\
\text { disaster } \\
1 \text { year } \\
\text { post disaster } \\
2 \text { years } \\
\text { postdisaster }\end{array}$ & $\begin{array}{l}\text { Depressive } \\
\text {, Anxious } \\
\text { PTSS }\end{array}$ \\
\hline $\begin{array}{l}\text { Roussos, A., } \\
\text { Goenjian, A. } \\
\text { K., Steinberg, } \\
\text { A. M., } \\
\text { Sotiropoulou, } \\
\text { C., Kakaki, } \\
\text { M., Kabakos, } \\
\text { C., ... \& } \\
\text { Manouras, V. } \\
(2005) \text {. }\end{array}$ & $\begin{array}{l}\text { Earthquake } \\
\text { (Greece) }\end{array}$ & $\begin{array}{l}\text { Cross- } \\
\text { Sectional }\end{array}$ & Individual & $\begin{array}{l}1937 \\
\text { children and } \\
\text { adolescent } \\
\text { aged 9-18 } \\
\text { years, of } \\
\text { two } \\
\text { differentially } \\
\text { exposed } \\
\text { cities (ano } \\
\text { liosia, at the } \\
\text { epicenter, } \\
\text { and dafni, } 10 \\
\text { kilometers } \\
\text { from the } \\
\text { epicenter) }\end{array}$ & $\begin{array}{l}\text { Objective } \\
\text { and } \\
\text { subjective } \\
\text { features of } \\
\text { earthquake } \\
\text { exposure, } \\
\text { gender, } \\
\text { school level, } \\
\text { postearthqua } \\
\text { ke } \\
\text { difficulties, } \\
\text { death of a } \\
\text { family } \\
\text { member, } \\
\text { thoughts of } \\
\text { revenge }\end{array}$ & $\begin{array}{l}3 \text { months } \\
\text { post disaster }\end{array}$ & $\begin{array}{l}\text { Posttrauma } \\
\text { tic stress } \\
\text { and } \\
\text { depressive } \\
\text { reactions }\end{array}$ \\
\hline $\begin{array}{l}\text { Roysircar, G., } \\
\text { Colvin, K. F., } \\
\text { Afolayan, A. } \\
\text { G., } \\
\text { Thompson, } \\
\text { A., \& } \\
\text { Robertson, T. } \\
\text { W. } \\
(2017) \text {. }\end{array}$ & $\begin{array}{l}\text { Earthquake } \\
\text { (Haiti) }\end{array}$ & $\begin{array}{l}\text { Cross- } \\
\text { sectional }\end{array}$ & Individual & $\begin{array}{l}131 \text { children } \\
\text { and } \\
\text { adolescent } \\
\text { aged } 6-15 \\
\text { years }\end{array}$ & $\begin{array}{l}\text { Age, } \\
\text { gender, } \\
\text { year, } \\
\text { location }\end{array}$ & & $\begin{array}{l}\text { Resilience } \\
\text { and } \\
\text { vulnerabili } \\
\text { ty }\end{array}$ \\
\hline
\end{tabular}




\begin{tabular}{|c|c|c|c|c|c|c|c|}
\hline $\begin{array}{l}\text { Russell, J. D., } \\
\text { Neill, E. L., } \\
\text { Carrión, V. } \\
\text { G., \& Weems, } \\
\text { C. F. (2017). }\end{array}$ & $\begin{array}{l}\text { Hurricane } \\
\text { (USA) }\end{array}$ & $\begin{array}{l}\text { Cross- } \\
\text { sectional }\end{array}$ & Individual & $\begin{array}{l}786 \text { children } \\
\text { aged } 8-13 \\
\text { years and } \\
\text { adolescent } \\
14-18 \text { years }\end{array}$ & $\begin{array}{l}\text { Severity } \\
\text { symptoms, } \\
\text { symptom } \\
\text { indices }\end{array}$ & $\begin{array}{l}36 \text { months } \\
\text { post disaster }\end{array}$ & $\begin{array}{l}\text { Network } \\
\text { structure of } \\
\text { PTSD }\end{array}$ \\
\hline $\begin{array}{l}\text { Russoniello, } \\
\text { C. V., Skalko, } \\
\text { T. K., O'brien, } \\
\text { K., McGhee, } \\
\text { S. A., } \\
\text { Bingham- } \\
\text { Alexander, } \\
\text { D., \& Beatley, } \\
\text { J. (2002). }\end{array}$ & $\begin{array}{l}\text { Hurricane } \\
\text { and } \\
\text { Flood } \\
\text { (USA) }\end{array}$ & $\begin{array}{l}\text { Cross- } \\
\text { Sectional }\end{array}$ & $\begin{array}{l}\text { Individual, } \\
\text { Interpersona } \\
1 \\
\text { and } \\
\text { Intergroup }\end{array}$ & $\begin{array}{l}218 \text { children } \\
\text { aged } 9-12 \\
\text { years }\end{array}$ & $\begin{array}{l}\text { Experiencin } \\
\text { g flooding at } \\
\text { home, } \\
\text { gender, } \\
\text { coping, } \\
\text { social } \\
\text { support, } \\
\text { race }\end{array}$ & $\begin{array}{l}6 \text { months } \\
\text { post disaster }\end{array}$ & $\begin{array}{l}\text { PTSD } \\
\text { symptom }\end{array}$ \\
\hline $\begin{array}{l}\text { Şahin, N. H., } \\
\text { Batıün, A. } \\
\text { D., \& Yilmaz, } \\
\text { B. (2007). }\end{array}$ & $\begin{array}{l}\text { Earthquake } \\
\text { (Turkey) }\end{array}$ & $\begin{array}{l}\text { Longitudin } \\
\text { al }\end{array}$ & $\begin{array}{l}\text { Individual } \\
\text { and } \\
\text { Interpersona } \\
1\end{array}$ & $\begin{array}{l}\text { Study 1: } \\
420 \text { children } \\
\text { aged 6-11 } \\
\text { years } \\
\text { Study 2: } \\
948 \\
\text { adolescents } \\
\text { aged } 12-16 \\
\text { years }\end{array}$ & $\begin{array}{l}\text { Gender, } \\
\text { location, } \\
\text { impact of the } \\
\text { disaster, } \\
\text { separation } \\
\text { from } \\
\text { family, } \\
\text { losses, } \\
\text { injuries, } \\
\text { disaster } \\
\text { exposure, } \\
\text { school } \\
\text { performance } \\
\text {, } \\
\text { reasons for } \\
\text { living, } \\
\text { future } \\
\text { expectation }\end{array}$ & $\begin{array}{l}5 \text { months } \\
\text { post second } \\
\text { earthquake } \\
\text { (T1) } \\
3 \text { months after } \\
\text { the first data } \\
\text { Collection } \\
\text { (T2) }\end{array}$ & $\begin{array}{l}\text { PTSS } \\
\text { Psychologi } \\
\text { cal } \\
\text { symptoms }\end{array}$ \\
\hline $\begin{array}{l}\text { Sales, J. M., } \\
\text { Fivush, R., } \\
\text { Parker, J., \& } \\
\text { Bahrick, L. } \\
(2005) .\end{array}$ & $\begin{array}{l}\text { Hurricane } \\
\text { (USA) }\end{array}$ & $\begin{array}{l}\text { Longitudin } \\
\text { al }\end{array}$ & Individual & $\begin{array}{l}35 \text { children } \\
\text { aged 3-4 } \\
\text { years }\end{array}$ & $\begin{array}{l}\text { Low, } \\
\text { moderate, } \\
\text { and high- } \\
\text { stress groups }\end{array}$ & $\begin{array}{l}\text { Immediately } \\
\text { post disaster } \\
\text { (T1) } \\
6 \text { years later } \\
\text { (T2) }\end{array}$ & $\begin{array}{l}\text { Recall } \\
\text { and PTSD }\end{array}$ \\
\hline $\begin{array}{l}\text { Salloum, A., } \\
\text { \& Lewis, M. } \\
\text { L. (2010). }\end{array}$ & $\begin{array}{l}\text { Hurricane } \\
\text { (USA) }\end{array}$ & $\begin{array}{l}\text { Cross- } \\
\text { sectional }\end{array}$ & $\begin{array}{l}\text { Individual } \\
\text { and }\end{array}$ & $\begin{array}{l}42 \text { African } \\
\text { American } \\
\text { children }\end{array}$ & $\begin{array}{l}\text { Disaster } \\
\text { exposure, }\end{array}$ & $\begin{array}{l}\text { 5-8 months } \\
\text { post disaster }\end{array}$ & $\begin{array}{l}\text { Children's } \\
\text { coping } \\
\text { strategy }\end{array}$ \\
\hline
\end{tabular}




\begin{tabular}{|c|c|c|c|c|c|c|c|c|c|}
\hline & & & $\begin{array}{l}\text { Interpersona } \\
1\end{array}$ & $\begin{array}{l}\text { aged } 7-12 \\
\text { years and } \\
38 \text { parents }\end{array}$ & $\begin{array}{l}\text { parent's } \\
\text { coping } \\
\text { strategy }\end{array}$ & & & & \\
\hline $\begin{array}{l}\text { Salloum, A., } \\
\text { Carter, P., } \\
\text { Burch, B., } \\
\text { Garfinkel, A., } \\
\text { \& Overstreet, } \\
\text { S. (2011). }\end{array}$ & $\begin{array}{l}\text { Hurricane } \\
\text { and } \\
\text { Exposure } \\
\text { to } \\
\text { Communit } \\
\text { y Violence } \\
\text { (USA) }\end{array}$ & $\begin{array}{l}\text { Cross- } \\
\text { Sectional }\end{array}$ & $\begin{array}{l}\text { Individual } \\
\text { and } \\
\text { Interpersona } \\
1\end{array}$ & $\begin{array}{l}122 \text { children } \\
\text { aged } 7-12 \\
\text { years }\end{array}$ & $\begin{array}{l}\text { Disaster } \\
\text { exposure }\end{array}$ & & $\begin{array}{l}\text { Prior } \\
\text { experiences } \\
\text { with } \\
\text { hurricane } \\
\text { Katrina } \\
\text { Exposure to } \\
\text { community } \\
\text { violence }\end{array}$ & & $\begin{array}{l}\text { PTSS and } \\
\text { depression }\end{array}$ \\
\hline $\begin{array}{l}\text { Saylor, C. F., } \\
\text { Swenson, C. } \\
\text { C., Stokes } \\
\text { Reynolds, S., } \\
\text { \& Taylor, M. } \\
(1999) .\end{array}$ & $\begin{array}{l}\text { Hurricane } \\
\text { (USA) }\end{array}$ & $\begin{array}{l}\text { Cross- } \\
\text { Sectional }\end{array}$ & Individual & $\begin{array}{l}475 \text { children } \\
\text { aged } 2-10 \\
\text { years }\end{array}$ & $\begin{array}{l}\text { Disaster } \\
\text { exposure, } \\
\text { different } \\
\text { type of } \\
\text { trauma, } \\
\text { age, } \\
\text { gender }\end{array}$ & & & $\begin{array}{l}14 \text { months } \\
\text { post disaster }\end{array}$ & $\begin{array}{l}\text { PTSD } \\
\text { Emotional } \\
\text { distress }\end{array}$ \\
\hline $\begin{array}{l}\text { Scaramella, L. } \\
\text { V., Sohr- } \\
\text { Preston, S. L., } \\
\text { Callahan, K. } \\
\text { L., \& } \\
\text { Mirabile, S. P. } \\
(2008) \text {. }\end{array}$ & $\begin{array}{l}\text { Hurricane } \\
\text { (USA) }\end{array}$ & $\begin{array}{l}\text { Pre-post } \\
\text { and } \\
\text { longitudina } \\
1\end{array}$ & $\begin{array}{l}\text { Individual } \\
\text { and } \\
\text { Interpersona } \\
1\end{array}$ & $\begin{array}{l}2 \text { groups of } \\
\text { low-income } \\
\text { mothers and } \\
\text { their } \\
\text { children } \\
\text { aged } 2 \text { years } \\
\text { pre-disaster: } \\
n=55 \\
\text { post- } \\
\text { disaster: } \\
n=47\end{array}$ & & $\begin{array}{l}\text { Mother's } \\
\text { depression } \\
\text { Parenting } \\
\text { efficacy } \\
\text { Perceived } \\
\text { financial } \\
\text { strain, } \\
\text { Neighborh } \\
\text { ood } \\
\text { violence } \\
\text { Number of } \\
\text { adults in } \\
\text { the home }\end{array}$ & & $\begin{array}{l}1 \text { to } 2 \text { years } \\
\text { pre disaster } \\
\text { (T1) } \\
6 \text { months } \\
\text { post disaster } \\
\text { (T2) } \\
18 \text { months } \\
\text { post disaster } \\
\text { (T3) }\end{array}$ & $\begin{array}{l}\text { Child } \\
\text { problem } \\
\text { behaviour }\end{array}$ \\
\hline
\end{tabular}




\begin{tabular}{|c|c|c|c|c|c|c|c|}
\hline $\begin{array}{l}\text { Scheeringa, } \\
\text { M. S. (2014). }\end{array}$ & $\begin{array}{l}\text { Olis Spill } \\
\text { and } \\
\text { Hurricane } \\
\text { Katrina } \\
\text { (USA) }\end{array}$ & $\begin{array}{l}\text { Cross- } \\
\text { Sectional }\end{array}$ & Individual & $\begin{array}{l}284 \text { children } \\
\text { aged } 3-6 \\
\text { years }\end{array}$ & $\begin{array}{l}\text { Type of } \\
\text { disaster: } \\
\text { oils spill, } \\
\text { disaster } \\
\text { exposure } \\
\text { repeated } \\
\text { traumas } \\
\text { (cumulative } \\
\text { number of } \\
\text { events) }\end{array}$ & & PTSD \\
\hline $\begin{array}{l}\text { Scheeringa, } \\
\text { M. S., \& } \\
\text { Zeanah, C. H. } \\
(2008) .\end{array}$ & $\begin{array}{l}\text { Hurricane } \\
\text { (USA) }\end{array}$ & $\begin{array}{l}\text { Cross- } \\
\text { Sectional }\end{array}$ & $\begin{array}{l}\text { Individual } \\
\text { and } \\
\text { Interpersona } \\
1\end{array}$ & $\begin{array}{l}70 \text { preschool } \\
\text { children } \\
\text { ages } 3-6 \\
\text { and their } \\
\text { caregiver }\end{array}$ & $\begin{array}{l}\text { Disaster } \\
\text { exposure } \\
\text { and } \\
\text { experiences, } \\
\text { groups } \\
\text { (stayed/evac } \\
\text { uated, } \\
\text { separated/no } \\
\text { t separated, } \\
\text { black/non- } \\
\text { black and } \\
\text { boy/girl }\end{array}$ & & $\begin{array}{l}\text { PTSD } \\
\text { Comorbid } \\
\text { Disorders }\end{array}$ \\
\hline $\begin{array}{l}\text { Schwind, J. } \\
\text { S., Formby, } \\
\text { C. B., } \\
\text { Santangelo, S. } \\
\text { L., Norman, } \\
\text { S. A., Brown, } \\
\text { R., Hoffman } \\
\text { Frances, R., } \\
\text {... } \\
\text { Karmacharya, } \\
\text { D. (2018) }\end{array}$ & $\begin{array}{l}\text { Earthquake } \\
\text { (Nepal) }\end{array}$ & $\begin{array}{l}\text { Cross- } \\
\text { sectional }\end{array}$ & $\begin{array}{l}\text { Individual } \\
\text { Interpersona } \\
1 \\
\text { Intergroup }\end{array}$ & $\begin{array}{l}62 \text { children } \\
8-17 \text { years } \\
\text { old and } \\
\text { parents }\end{array}$ & $\begin{array}{l}\text { Earthquake } \\
\text { exposures } \\
\text { age } \\
\text { gender } \\
\text { marital } \\
\text { status } \\
\text { household } \\
\text { identifed } \\
\text { religion } \\
\text { household } \\
\text { identifed } \\
\text { ethnic } \\
\text { group } \\
\text { level of } \\
\text { education } \\
\text { occupation }\end{array}$ & $\begin{array}{l}1 \text { year after } \\
\text { disaster }\end{array}$ & $\begin{array}{l}\text { Depression } \\
\text { PTSD } \\
\text { Resilience }\end{array}$ \\
\hline
\end{tabular}




\section{RESILIENCE IN CHILDREN AFTER NATURAL DISASTERS}

\begin{tabular}{|c|c|c|c|c|c|c|c|}
\hline & & & & & $\begin{array}{l}\text { number of } \\
\text { members in } \\
\text { household }\end{array}$ & & \\
\hline $\begin{array}{l}\text { Self-Brown, } \\
\text { S., Lai, B. S., } \\
\text { Harbin, S., \& } \\
\text { Kelley, M. L. } \\
(2014) .\end{array}$ & $\begin{array}{l}\text { Hurricane } \\
\text { (USA) }\end{array}$ & $\begin{array}{l}\text { Longitudin } \\
\text { al }\end{array}$ & $\begin{array}{l}\text { Individual } \\
\text { and } \\
\text { Interpersona } \\
1\end{array}$ & $\begin{array}{l}423 \text { mother- } \\
\text { child dyads } \\
\text { Children } \\
\text { aged } \\
8-16 \text { years }\end{array}$ & $\begin{array}{l}\text { Maternal } \\
\text { posttraumati } \\
\text { c stress } \\
\text { disorder } \\
\text { symptom } \\
\text { trajectories }\end{array}$ & $\begin{array}{l}\text { 3-7 months } \\
\text { post disaster } \\
\text { (T1) } \\
\text { 13-17months } \\
\text { post disaster } \\
\text { (T2) } \\
\text { 19-22 months } \\
\text { post disaster } \\
\text { (T3) } \\
25-27 \text { months } \\
\text { post disaster } \\
\text { (T4) }\end{array}$ & PTSS \\
\hline $\begin{array}{l}\text { Shannon, M. } \\
\text { P., Lonigan, } \\
\text { C. J., Finch Jr, } \\
\text { A. J., \& } \\
\text { Taylor, C. M. } \\
\text { (1994). }\end{array}$ & $\begin{array}{l}\text { Hurricane } \\
\text { (USA) }\end{array}$ & $\begin{array}{l}\text { Cross- } \\
\text { sectional }\end{array}$ & $\begin{array}{l}\text { Individual } \\
\text { and } \\
\text { Intergroup }\end{array}$ & $\begin{array}{l}5687 \\
\text { school-aged } \\
\text { children } \\
\text { aged 9-13 } \\
\text { years }\end{array}$ & $\begin{array}{l}\text { Disaster } \\
\text { exposure, } \\
\text { race, } \\
\text { gender, } \\
\text { age }\end{array}$ & $\begin{array}{l}3 \text { months } \\
\text { post disaster }\end{array}$ & $\begin{array}{l}\text { PTSD } \\
\text { School } \\
\text { performan } \\
\text { ce }\end{array}$ \\
\hline $\begin{array}{l}\text { Shaw, J. A., } \\
\text { Applegate, B., } \\
\text { \& Schorr, C. } \\
\text { (1996). }\end{array}$ & $\begin{array}{l}\text { Hurricane } \\
\text { (USA) }\end{array}$ & $\begin{array}{l}\text { Longitudin } \\
\text { al }\end{array}$ & Individual & $\begin{array}{l}30 \text { school } \\
\text { children } \\
\text { aged } \\
7-13 \text { years }\end{array}$ & $\begin{array}{l}\text { Disaster } \\
\text { exposure, } \\
\text { gender }\end{array}$ & $\begin{array}{l}2 \text { months } \\
\text { post disaster } \\
\text { (T1) } \\
8 \text { months } \\
\text { post disaster } \\
\text { (T2) } \\
21 \text { months } \\
\text { post disaster } \\
\text { (T3) }\end{array}$ & $\begin{array}{l}\text { PTSS } \\
\text { and } \\
\text { psychologi } \\
\text { cal } \\
\text { morbidity }\end{array}$ \\
\hline $\begin{array}{l}\text { Shaw, J. A., } \\
\text { Applegate, B., } \\
\text { Tanner, S., } \\
\text { Perez, D., } \\
\text { Rothe, E., }\end{array}$ & $\begin{array}{l}\text { Hurricane } \\
\text { (USA) }\end{array}$ & $\begin{array}{l}\text { Longitudin } \\
\text { al }\end{array}$ & Individual & $\begin{array}{l}106 \text { school } \\
\text { children } \\
\text { aged } \\
6-11 \text { years }\end{array}$ & $\begin{array}{l}\text { High and } \\
\text { low impact } \\
\text { groups }\end{array}$ & $\begin{array}{l}8 \text { weeks } \\
\text { post disaster } \\
\text { (T1) } \\
32 \text { weeks }\end{array}$ & PTSS \\
\hline
\end{tabular}




\begin{tabular}{|c|c|c|c|c|c|c|c|c|}
\hline $\begin{array}{l}\text { Campo- } \\
\text { Bowen, A. E., } \\
\text { \& Lahey, B. } \\
\text { L. (1995). }\end{array}$ & & & & & & & $\begin{array}{l}\text { post disaster } \\
\text { (T2) }\end{array}$ & \\
\hline $\begin{array}{l}\text { Silwal, S., } \\
\text { Dybdahl, R., } \\
\text { Chudal, R., } \\
\text { Sourander, A., } \\
\text { \& Lien, L. } \\
\text { (2018). }\end{array}$ & $\begin{array}{l}\text { Earthquake } \\
\text { (Nepal) }\end{array}$ & $\begin{array}{l}\text { Cross- } \\
\text { Sectional }\end{array}$ & Individual & $\begin{array}{l}893 \text { students } \\
\text { aged } 11-17 \\
\text { years }\end{array}$ & $\begin{array}{l}\text { Age, } \\
\text { gender, } \\
\text { ethnicity, } \\
\text { parent's } \\
\text { education } \\
\text { and prior } \\
\text { exposure to } \\
\text { trauma, } \\
\text { disaster } \\
\text { exposure } \\
\text { and } \\
\text { experience }\end{array}$ & Gender & $\begin{array}{l}12 \text { months } \\
\text { post disaster }\end{array}$ & $\begin{array}{l}\text { PTSS } \\
\text { and } \\
\text { depressive } \\
\text { symptoms }\end{array}$ \\
\hline $\begin{array}{l}\text { Simcock, G., } \\
\text { Cobham, V. } \\
\text { E., Laplante, } \\
\text { D. P., } \\
\text { Elgbeili, G., } \\
\text { Gruber, R., } \\
\text { Kildea, S., \& } \\
\text { King, S. } \\
\text { (2019) }\end{array}$ & $\begin{array}{l}\text { Flood } \\
\text { (USA) }\end{array}$ & $\begin{array}{l}\text { Longitudin } \\
\text { al }\end{array}$ & $\begin{array}{l}\text { Individual } \\
\text { Interpersona } \\
1\end{array}$ & $\begin{array}{l}86 \text { mother } \\
\text { and children, } \\
3-4 \text { years old }\end{array}$ & $\begin{array}{l}\text { Prenatal } \\
\text { maternal } \\
\text { stress } \\
\text { Maternal } \\
\text { marital } \\
\text { status, } \\
\text { socioeconom } \\
\text { ic status } \\
\text { and } \\
\text { education } \\
\text { level } \\
\text { Maternal } \\
\text { cognitive } \\
\text { appraisal }\end{array}$ & & $\begin{array}{l}12 \\
\text { months post- } \\
\text { flood } \\
30 \text { months and } \\
48 \text { month after } \\
\text { flood }\end{array}$ & $\begin{array}{l}\text { sleep, } \\
\text { attention } \\
\text { anxious/de } \\
\text { pressed } \\
\text { symptoms }\end{array}$ \\
\hline $\begin{array}{l}\text { Simcock, G., } \\
\text { Elgbeili, G., } \\
\text { Laplante, D. } \\
\text { P., Kildea, S., } \\
\text { Cobham, V., } \\
\text { Stapleton, H., } \\
\text {... King, S. } \\
\text { (2017) }\end{array}$ & $\begin{array}{l}\text { Flood } \\
\text { (Queenslan } \\
\text { d) }\end{array}$ & $\begin{array}{l}\text { Cross- } \\
\text { sectional }\end{array}$ & $\begin{array}{l}\text { Individual } \\
\text { Intepersonal }\end{array}$ & $\begin{array}{l}126 \text { mother } \\
\text { and children } \\
6 \text { months old }\end{array}$ & $\begin{array}{l}\text { Prenatal } \\
\text { Maternal } \\
\text { Stress } \\
\text { Mather } \\
\text { exposure, } \\
\text { subjective } \\
\text { stress, } \\
\text { Cognitive } \\
\text { appraisal, } \\
\text { school level, }\end{array}$ & $\begin{array}{l}\text { infant sex, } \\
\text { timing of the } \\
\text { flood in } \\
\text { gestation, } \\
\text { and mother's } \\
\text { emotional } \\
\text { response to } \\
\text { the disaster. }\end{array}$ & $\begin{array}{l}3-10 \text { months } \\
\text { after flood }\end{array}$ & $\begin{array}{l}\text { Infant } \\
\text { temperame } \\
\text { nt }\end{array}$ \\
\hline
\end{tabular}


RESILIENCE IN CHILDREN AFTER NATURAL DISASTERS

\begin{tabular}{|c|c|c|c|c|c|c|c|}
\hline & & & & & $\begin{array}{l}\text { socioeconom } \\
\text { ic status, } \\
\text { anxiety, } \\
\text { depression, } \\
\text { gestational } \\
\text { age at birth } \\
\text { Infant sex }\end{array}$ & & \\
\hline $\begin{array}{l}\text { Simcock, G., } \\
\text { Laplante, D. } \\
\text { P., Elgbeili, } \\
\text { G., Kildea, S., } \\
\text { \& King, S. } \\
(2018)\end{array}$ & $\begin{array}{l}\text { Flood } \\
\text { (USA) }\end{array}$ & $\begin{array}{l}\text { Longitudin } \\
\text { al }\end{array}$ & $\begin{array}{l}\text { Individual } \\
\text { Interpersona } \\
1\end{array}$ & $\begin{array}{l}206 \text { mothers } \\
\text { and children } \\
\text { aged: } 2 \\
\text { Months } 6 \\
\text { Months } 16 \\
\text { Months } 2 \frac{1}{2} 2 \\
\text { Years } 4 \\
\text { Years }\end{array}$ & $\begin{array}{l}\text { Mothers' } \\
\text { objective } \\
\text { exposure } \\
\text { Mothers' } \\
\text { post- } \\
\text { traumatic } \\
\text { subjective } \\
\text { stress } \\
\text { Mothers' } \\
\text { peritraumati } \\
\text { c distress } \\
\text { Mothers' } \\
\text { Cognitive } \\
\text { appraisal } \\
\text { Age of } \\
\text { gestation } \\
\text { exposure to } \\
\text { the flood. } \\
\text { Maternal } \\
\text { depression } \\
\text { and anxiety } \\
\text { Maternal } \\
\text { socioeconom } \\
\text { ic status } \\
\text { Maternal } \\
\text { education } \\
\text { level. } \\
\text { Child } \\
\text { gestational } \\
\text { age, birth } \\
\text { weight, and } \\
\text { head }\end{array}$ & $\begin{array}{l}2 \text { Months } 6 \\
\text { Months } 16 \\
\text { Months } 2 \frac{1}{2} \\
\text { Years } 4 \text { Years } \\
\text { after flood }\end{array}$ & $\begin{array}{l}\text { Child } \\
\text { motor } \\
\text { developme } \\
\text { nt }\end{array}$ \\
\hline
\end{tabular}




\section{RESILIENCE IN CHILDREN AFTER NATURAL DISASTERS}

\begin{tabular}{|c|c|c|c|c|c|c|c|c|}
\hline & & & & & $\begin{array}{l}\text { circumferenc } \\
\text { e }\end{array}$ & & & \\
\hline $\begin{array}{l}\text { Siqveland, J., } \\
\text { Hafstad, G. } \\
\text { S., \& } \\
\text { Tedeschi, R. } \\
\text { G. } \\
(2012) .\end{array}$ & $\begin{array}{l}\text { Tsunami } \\
\text { (Southeast } \\
\text { Asia) }\end{array}$ & $\begin{array}{l}\text { Longitudin } \\
\text { al }\end{array}$ & $\begin{array}{l}\text { Individual } \\
\text { and } \\
\text { Interpersona } \\
1\end{array}$ & $\begin{array}{l}68 \text { parents } \\
\text { and their } \\
105 \\
\text { norwegian } \\
\text { children }\end{array}$ & $\begin{array}{l}\text { Parent's } \\
\text { disaster } \\
\text { exposure, } \\
\text { child- } \\
\text { disaster } \\
\text { exposure }\end{array}$ & & $\begin{array}{l}6 \text { months } \\
\text { post disaster } \\
\text { (T1) } \\
10 \text { months } \\
\text { post disaster } \\
\text { (T2) } \\
30 \text { months } \\
\text { post disaster } \\
\text { (T3) }\end{array}$ & $\begin{array}{l}\text { PTG in } \\
\text { parents } \\
\text { PTSS in } \\
\text { parents and } \\
\text { children }\end{array}$ \\
\hline $\begin{array}{l}\text { Siswa } \\
\text { Widyatmoko, } \\
\text { C., Tan, E. T., } \\
\text { Conor Seyle, } \\
\text { D., Haksi } \\
\text { Mayawati, E., } \\
\text { \& Cohen } \\
\text { Silver, R. } \\
(2011) .\end{array}$ & $\begin{array}{l}\text { Earthquake } \\
\text { (Indonesia) }\end{array}$ & $\begin{array}{l}\text { Cross- } \\
\text { sectional }\end{array}$ & Individual & $\begin{array}{l}3115 \\
\text { children } \\
\text { aged 6-14 } \\
\text { years } \\
\text { and teachers }\end{array}$ & $\begin{array}{l}\text { Children } \\
\text { symptoms } \\
\text { identified } \\
\text { from } \\
\text { teachers, }\end{array}$ & & $\begin{array}{l}2 \text { years } \\
\text { post disaster }\end{array}$ & PTSS \\
\hline $\begin{array}{l}\text { Soysa, C. K. } \\
(2013) .\end{array}$ & $\begin{array}{l}\text { Tsunami } \\
\text { War } \\
\text { (Sri Lanka) }\end{array}$ & $\begin{array}{l}\text { Longitudin } \\
\text { al }\end{array}$ & & $\begin{array}{l}\text { War sample: } \\
60 \text { youths } \\
\text { aged 9-16 } \\
\text { years olds } \\
\text { Tsunami } \\
\text { sample: } \\
60 \\
\text { adolescent } \\
\text { aged } 12-14 \\
\text { years }\end{array}$ & $\begin{array}{l}\text { Trauma } \\
\text { exposure } \\
\text { (war } \\
\text { or tsunami) }\end{array}$ & & $\begin{array}{l}3 \text { months } \\
\text { post a major } \\
\text { war-related } \\
\text { event } \\
3 \text { months post } \\
\text { tsunami }\end{array}$ & PTSD \\
\hline
\end{tabular}




\section{RESILIENCE IN CHILDREN AFTER NATURAL DISASTERS}

\begin{tabular}{|c|c|c|c|c|c|c|c|c|}
\hline $\begin{array}{l}\text { Self-Brown, } \\
\text { S., Davidson, } \\
\text { K. L., } \\
\text { Pellegrin, A., } \\
\text {.. \& } \\
\text { Baumeister, } \\
\text { A. } \\
(2008) .\end{array}$ & & & $\begin{array}{l}\text { Interpersona } \\
1\end{array}$ & $\begin{array}{l}\text { their } \\
\text { mothers }\end{array}$ & & $\begin{array}{l}1 \text { distress and } \\
\text { maternal } \\
\text { posttraumati } \\
\text { c stress } \\
\text { disorder }\end{array}$ & & $\begin{array}{l}\text { distress: } \\
\text { child } \\
\text { internalizin } \\
\text { g and } \\
\text { externalizi } \\
\text { ng } \\
\text { symptoms }\end{array}$ \\
\hline $\begin{array}{l}\text { Sprung, M. } \\
(2008) .\end{array}$ & $\begin{array}{l}\text { Hurricane } \\
\text { (USA) }\end{array}$ & $\begin{array}{l}\text { Cross- } \\
\text { sectional }\end{array}$ & Individual & $\begin{array}{l}183 \text { children } \\
\text { aged 5-8 } \\
\text { years: } \\
145 \text { from the } \\
\text { hurricane } \\
\text { Katrina } \\
\text { disaster area, } \\
95 \text { from } \\
\text { Coastal } \\
\text { Mississippi, } \\
\text { and } \\
50 \text { children } \\
\text { were from } \\
\text { Hattiesburg, } \\
\text { Mississippi. } \\
38 \text { children } \\
\text { composing } \\
\text { the control } \\
\text { group from } \\
\text { Boston area. }\end{array}$ & $\begin{array}{l}\text { Gender, } \\
\text { age, } \\
\text { ethnicity, } \\
\text { socioeconom } \\
\text { ic resources, } \\
\text { higher or } \\
\text { low levels of } \\
\text { understandin } \\
\text { g of the } \\
\text { mind }\end{array}$ & & $\begin{array}{l}7 \text { months } \\
\text { post disaster }\end{array}$ & $\begin{array}{l}\text { Intrusive } \\
\text { thoughts } \\
\text { and } \\
\text { level of } \\
\text { cognitive } \\
\text { functionin } \\
\mathrm{g}\end{array}$ \\
\hline $\begin{array}{l}\text { Sprung, M., \& } \\
\text { Harris, P. L. } \\
(2010) .\end{array}$ & $\begin{array}{l}\text { Hurricane } \\
\text { (USA) }\end{array}$ & $\begin{array}{l}\text { Cross- } \\
\text { Sectional }\end{array}$ & Individual & $\begin{array}{l}165 \text { children } \\
\text { aged } 5-9 \\
\text { years }\end{array}$ & $\begin{array}{l}\text { Level of } \\
\text { exposure: } \\
\text { proximity } \\
\text { to the } \\
\text { hurricane } \\
\text { and the loss- } \\
\text { disruption }\end{array}$ & & $\begin{array}{l}7 \text { months } \\
\text { post disaster }\end{array}$ & $\begin{array}{l}\begin{array}{l}\text { Intrusive } \\
\text { thoughts }\end{array} \\
\text { Attention } \\
\text { Knowledg } \\
\text { e about } \\
\text { thinking: } \\
\text { i.e., }\end{array}$ \\
\hline
\end{tabular}




\begin{tabular}{|c|c|c|c|c|c|c|c|c|}
\hline & & & & & & & & $\begin{array}{l}\text { theory-of- } \\
\text { mind or } \\
\text { meta- } \\
\text { cognitive } \\
\text { competenc } \\
\text { ies } \\
\text { General } \\
\text { language } \\
\text { abilities }\end{array}$ \\
\hline $\begin{array}{l}\text { Stoppelbein, } \\
\text { L., \& } \\
\text { Greening, L. } \\
(2000) .\end{array}$ & $\begin{array}{l}\text { Tornado } \\
\text { (n.d.) }\end{array}$ & $\begin{array}{l}\text { Experiment } \\
\text { al } \\
\text { (with } \\
\text { control } \\
\text { group) }\end{array}$ & Individual & $\begin{array}{l}226 \text { children } \\
\text { and } \\
\text { adolescents } \\
\text { aged } 7-17 \\
\text { years: } \\
\text { - } 39 \\
\text { bereaved } \\
\text { group } \\
\text {-118 disaster } \\
\text { group } \\
\text { - } 69 \text { non- } \\
\text { trauma } \\
\text { control } \\
\text { group }\end{array}$ & $\begin{array}{l}\text { Age, gender, } \\
\text { type of } \\
\text { stressors: } \\
\text { (bereved, } \\
\text { disaster), } \\
\text { daily } \\
\text { stressors, } \\
\text { parent PTSD }\end{array}$ & & $\begin{array}{l}\text { Tornado group } \\
1 \text { year } \\
\text { post disaster } \\
\text { bereaved } \\
\text { group } \\
\text { an average of } \\
3 \text { years } \\
\text { post their loss }\end{array}$ & $\begin{array}{l}\text { PTSD } \\
\text { Emotional } \\
\text { adjustment } \\
\text { Depression }\end{array}$ \\
\hline
\end{tabular}




\section{RESILIENCE IN CHILDREN AFTER NATURAL DISASTERS}

\begin{tabular}{|c|c|c|c|c|c|c|c|}
\hline $\begin{array}{l}\text { A., Larson, K. } \\
\text { J., Roemmich, } \\
\text { J. N., \& } \\
\text { Hilmert, C. J. } \\
(2020)\end{array}$ & & & & & $\begin{array}{l}\text { Socioecono } \\
\text { mic status } \\
\text { maternal } \\
\text { age, child } \\
\text { sex }\end{array}$ & & $\begin{array}{l}\text { on in } \\
\text { children }\end{array}$ \\
\hline $\begin{array}{l}\text { Sullivan, M. } \\
\text { A., Saylor, C. } \\
\text { F., \& Foster, } \\
\text { K. Y. (1991). }\end{array}$ & $\begin{array}{l}\text { Hurricane } \\
\text { (USA) }\end{array}$ & $\begin{array}{l}\text { Pre-post } \\
\text { (without } \\
\text { control } \\
\text { group) }\end{array}$ & $\begin{array}{l}\text { Individual } \\
\text { and } \\
\text { Interpersona } \\
1\end{array}$ & $\begin{array}{l}238 \text { families } \\
278 \text { children } \\
\text { aged } 1,11- \\
6,3 \text { years }\end{array}$ & $\begin{array}{l}\text { Pre-post } \\
\text { hurricane } \\
\text { behavioural } \\
\text { problems }\end{array}$ & $\begin{array}{l}6-8 \text { weeks } \\
\text { post disaster }\end{array}$ & $\begin{array}{l}\text { Post- } \\
\text { hurricane } \\
\text { adjustment } \\
\text { of pre- } \\
\text { schoolers }\end{array}$ \\
\hline $\begin{array}{l}\text { Sun, X. Y., } \\
\text { Fan, H. M., } \\
\text { Bai, B., Song, } \\
\text { H. T., Tao, F. } \\
\text { Y., Song, Z. } \\
\text { X., ... \& } \\
\text { Zhang, L. Y. } \\
\text { (2014). }\end{array}$ & $\begin{array}{l}\text { Earthquake } \\
\text { (China) }\end{array}$ & $\begin{array}{l}\text { Cross- } \\
\text { Sectional }\end{array}$ & Individual & $\begin{array}{l}1828 \\
\text { children } \\
\text { aged 6-16 } \\
\text { years }\end{array}$ & $\begin{array}{l}\text { Affected vs. } \\
\text { non-affected } \\
\text { group, age, } \\
\text { gender }\end{array}$ & $\begin{array}{l}2 \text { weeks } \\
\text { post disaster }\end{array}$ & $\begin{array}{l}\text { Psychosom } \\
\text { atic } \\
\text { symptoms }\end{array}$ \\
\hline $\begin{array}{l}\text { Sun, Y., \& } \\
\text { Yan, T. } \\
(2020)\end{array}$ & $\begin{array}{l}\text { Earthquake } \\
\text { Tsunami } \\
\text { (Japan) }\end{array}$ & $\begin{array}{l}\text { Cross- } \\
\text { sectional }\end{array}$ & $\begin{array}{l}\text { Individual } \\
\text { Interpersona } \\
1 \\
\text { Intergroup }\end{array}$ & $\begin{array}{l}498 \text { parents } \\
35 \text { children }\end{array}$ & $\begin{array}{l}\text { City of } \\
\text { residence } \\
\text { Age } \\
\text { Marital } \\
\text { status } \\
\text { Household } \\
\text { composition } \\
\text { Gender } \\
\text { Education } \\
\text { Economic } \\
\text { status } \\
\text { Impact of } \\
\text { event } \\
\text { Peer support }\end{array}$ & $\begin{array}{l}4 \text { years post } \\
\text { disaster }\end{array}$ & $\begin{array}{l}\text { mental } \\
\text { health }\end{array}$ \\
\hline
\end{tabular}




\section{RESILIENCE IN CHILDREN AFTER NATURAL DISASTERS}

\begin{tabular}{|c|c|c|c|c|c|c|c|c|}
\hline $\begin{array}{l}\text { Y., \& Belter, } \\
\text { R. W. (1996). }\end{array}$ & & & & $\begin{array}{l}170 \text { children } \\
\text { aged } 2-10 \\
\text { years no- } \\
\text { trauma } \\
\text { group } \\
\text { with } \\
\text { classroom } \\
\text { behavior } \\
\text { problems }\end{array}$ & & & & $\begin{array}{l}\text { Anxiety } \\
\text { and } \\
\text { withdrawal }\end{array}$ \\
\hline $\begin{array}{l}\text { Takada, S. } \\
\text { (2013). }\end{array}$ & $\begin{array}{l}\text { Earthquake } \\
\text { (Japan) }\end{array}$ & $\begin{array}{l}\text { Cross- } \\
\text { Sectional }\end{array}$ & Individual & $\begin{array}{l}8000 \\
\text { families with } \\
\text { pre-school } \\
\text { children and } \\
466 \text { families } \\
\text { with } \\
\text { disabled } \\
\text { children } \\
\text { Aged 4-6 } \\
\text { years }\end{array}$ & $\begin{array}{l}\text { Disaster } \\
\text { exposure, } \\
\text { house } \\
\text { damage, } \\
\text { disabilities }\end{array}$ & & $\begin{array}{l}12 \text { months } \\
\text { post disaster }\end{array}$ & PTSD \\
\hline $\begin{array}{l}\text { Tang, W., Lu, } \\
\text { Y., \& Xu, J. } \\
(2018)\end{array}$ & $\begin{array}{l}\text { Earthquake } \\
\text { (China) }\end{array}$ & $\begin{array}{l}\text { Cross- } \\
\text { sectional }\end{array}$ & Individual & $\begin{array}{l}6132 \\
\text { children 9- } \\
18 \text { years old }\end{array}$ & $\begin{array}{l}\text { Sleeping } \\
\text { problems } \\
\text { Negative life } \\
\text { events } \\
\text { Earthquake } \\
\text { exposure } \\
\text { Age } \\
\text { Gender }\end{array}$ & & $\begin{array}{l}3 \text { years after } \\
\text { earthquake }\end{array}$ & $\begin{array}{l}\text { PTSD } \\
\text { Depression } \\
\text { Anxiety }\end{array}$ \\
\hline $\begin{array}{l}\text { Tang, W., Xu, } \\
\text { D., Li, B., Lu, } \\
\text { Y., \& Xu, J. } \\
(2018)\end{array}$ & $\begin{array}{l}\text { Earthquake } \\
\text { (China) }\end{array}$ & $\begin{array}{l}\text { Cross- } \\
\text { sectional }\end{array}$ & Individual & $\begin{array}{l}5.563 \\
\text { children, 9- } \\
20 \text { years old }\end{array}$ & $\begin{array}{l}\text { Earthquake } \\
\text { exposure } \\
\text { Age } \\
\text { Gender }\end{array}$ & $\begin{array}{l}\text { depression } \\
\text { sleep } \\
\text { anxiety } \\
\text { PTSD }\end{array}$ & & $\begin{array}{l}\text { Suicidal } \\
\text { ideation }\end{array}$ \\
\hline $\begin{array}{l}\text { Tang, W., } \\
\text { Zhao, J., Lu, } \\
\text { Y., Yan, T., } \\
\text { Wang, L., } \\
\text { Zhang, J., \& } \\
\text { Xu, J. (2017). }\end{array}$ & $\begin{array}{l}\text { Earthquake } \\
\text { (China) }\end{array}$ & $\begin{array}{l}\text { Longitudin } \\
\text { al }\end{array}$ & $\begin{array}{l}\text { Individual, } \\
\text { Interpersona } \\
1 \\
\text { and } \\
\text { Intergroup }\end{array}$ & $\begin{array}{l}435 \text { children } \\
\text { age } M= \\
13,7 \text { years }\end{array}$ & $\begin{array}{l}\text { Age, } \\
\text { gender, } \\
\text { ethnic group, } \\
\text { school } \\
\text { grade, } \\
\text { disaster } \\
\text { exposure, }\end{array}$ & & $\begin{array}{l}12 \text { months } \\
\text { post disaster } \\
30 \text { months } \\
\text { post disaster }\end{array}$ & $\begin{array}{l}\text { PTSD } \\
\text { Depression } \\
\text { Anxiety }\end{array}$ \\
\hline
\end{tabular}




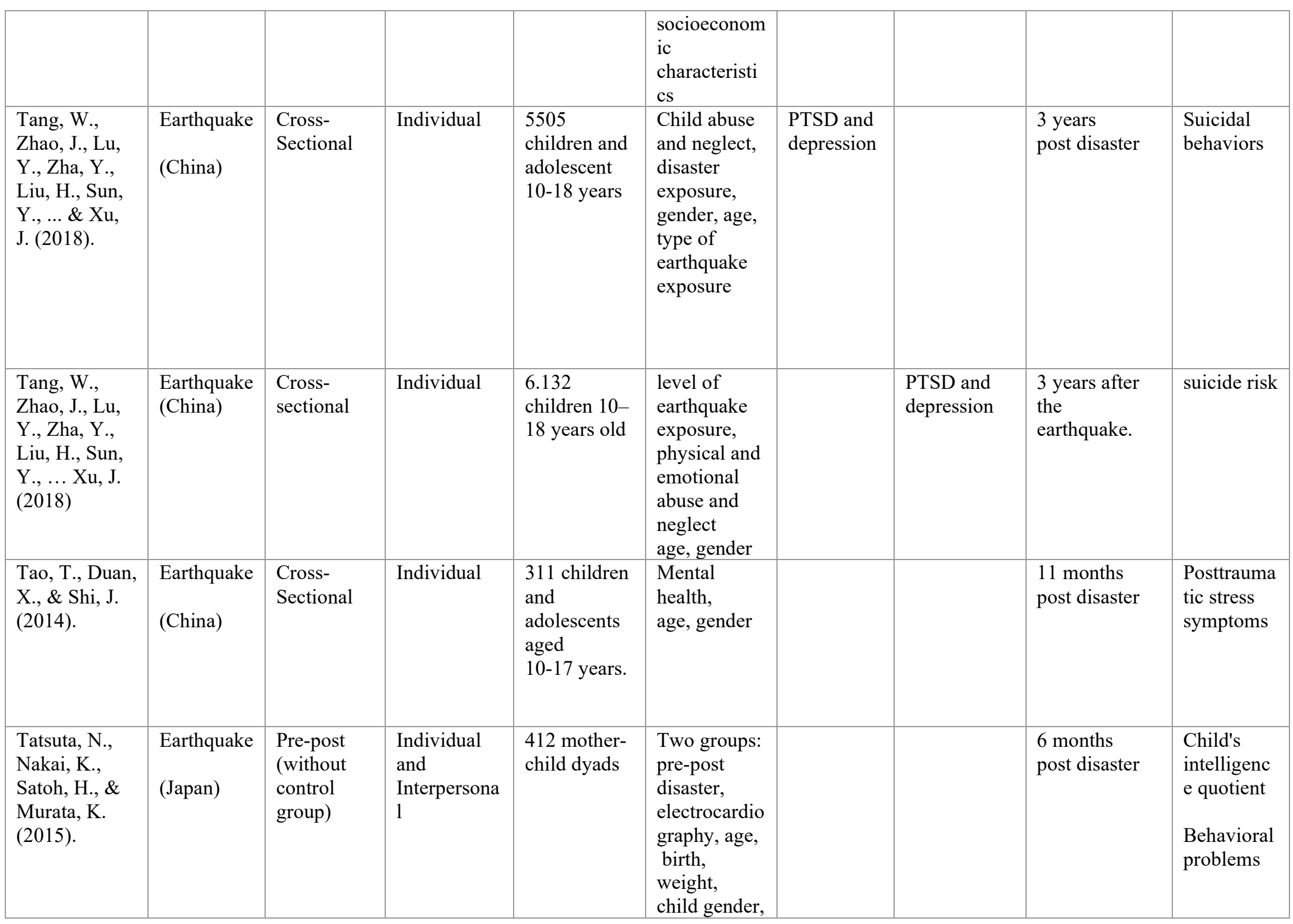




\begin{tabular}{|c|c|c|c|c|c|c|c|c|}
\hline & & & & & $\begin{array}{l}\text { maternal age } \\
\text { at } \\
\text { parturition, } \\
\text { birth order } \\
\text { delivery } \\
\text { type, } \\
\text { drinking and } \\
\text { smoking } \\
\text { habits during } \\
\text { pregnancy } \\
\text { maternal and } \\
\text { paternal } \\
\text { education, } \\
\text { levels and } \\
\text { annual } \\
\text { family } \\
\text { income, } \\
\text { cord blood } \\
\text { and maternal } \\
\text { blood, } \\
\text { home } \\
\text { environment }\end{array}$ & & & \\
\hline $\begin{array}{l}\text { Tees, M. T., } \\
\text { Harville, E. } \\
\text { W., Xiong, } \\
\text { X., Buekens, } \\
\text { P., Pridjian, } \\
\text { G., \& Elkind- } \\
\text { Hirsch, K. } \\
(2010) .\end{array}$ & $\begin{array}{l}\text { Hurricane } \\
\text { (USA) }\end{array}$ & $\begin{array}{l}\text { Longitudin } \\
\text { al }\end{array}$ & $\begin{array}{l}\text { Individual } \\
\text { and } \\
\text { Interpersona } \\
1\end{array}$ & 288 mothers & $\begin{array}{l}\text { Ddisaster } \\
\text { exposure, } \\
\text { maternal } \\
\text { mental } \\
\text { health: } \\
\text { - PTSD } \\
\text { - depression } \\
\text { post-partum } \\
\text { - hostility }\end{array}$ & & $\begin{array}{l}\begin{array}{l}\text { During the } \\
\text { disaster }\end{array} \\
2 \text { months } \\
\text { post disaster } \\
12 \text { months } \\
\text { postpartum }\end{array}$ & $\begin{array}{l}\text { Early } \\
\text { infant } \\
\text { temperame } \\
\text { nt } \\
\text { Activity, } \\
\text { adaptabilit } \\
\text { y, } \\
\text { approach, } \\
\text { intensity, } \\
\text { and mood } \\
\text { scales }\end{array}$ \\
\hline $\begin{array}{l}\text { Terranova, A. } \\
\text { M., Boxer, P., } \\
\text { \& Morris, A. } \\
\text { S. (2009). }\end{array}$ & $\begin{array}{l}\text { Hurricane } \\
\text { (USA) }\end{array}$ & $\begin{array}{l}\text { Longitudin } \\
\text { al }\end{array}$ & Individual & $\begin{array}{l}152 \text { children } \\
\text { ( } 23 \\
\text { participants } \\
\text { missing at } \\
\text { T2) }\end{array}$ & $\begin{array}{l}\text { Disaster } \\
\text { exposure, } \\
\text { gender and } \\
\text { ethnicity } \\
\text { peer }\end{array}$ & $\begin{array}{l}\text { Regulatory } \\
\text { abilities: } \\
\text { (effortful } \\
\text { control) }\end{array}$ & $\begin{array}{l}1.5 \text { months } \\
\text { post disaster } \\
\text { (T1) } \\
8 \text { months post } \\
\text { disaster }\end{array}$ & $\begin{array}{l}\text { PTSD (T1- } \\
\text { T2) }\end{array}$ \\
\hline
\end{tabular}




\begin{tabular}{|c|c|c|c|c|c|c|c|c|c|}
\hline & & & & $\begin{array}{l}\text { M age= } \\
11.52\end{array}$ & $\begin{array}{l}\text { victimization } \\
\text {, prosocial } \\
\text { behaviour, } \\
\text { fear } \\
\text { reactivity, } \\
\text { effortful } \\
\text { control, } \\
\text { negative } \\
\text { coping, } \\
\text { PTSD (t1) }\end{array}$ & & $\begin{array}{l}\text { Negative } \\
\text { coping }\end{array}$ & (T2) & \\
\hline $\begin{array}{l}\text { Terranova, A. } \\
\text { M., Morris, A. } \\
\text { S., Myers, S., } \\
\text { Kithakye, M., } \\
\text { \& Morris, M. } \\
\text { D. (2015). }\end{array}$ & $\begin{array}{l}\text { Hurricane } \\
\text { (USA) }\end{array}$ & $\begin{array}{l}\text { Pre-post } \\
\text { (without } \\
\text { experiment } \\
\text { al group) }\end{array}$ & $\begin{array}{l}\text { Individual } \\
\text { and } \\
\text { Interpersona } \\
1\end{array}$ & $\begin{array}{l}118 \text { children } \\
47 \text { aged } \\
\text { from } 4 \text { years } \\
\text { (pre and } \\
\text { post) } \\
71 \text { aged } \\
\text { from } \\
4-6 \text { years } \\
\text { (post) }\end{array}$ & $\begin{array}{l}\text { Family } \\
\text { functioning: } \\
\text { (parental } \\
\text { separation) } \\
\text { child } \\
\text { characteristi } \\
\text { cs, } \\
\text { disaster } \\
\text { exposure }\end{array}$ & $\begin{array}{l}\text { Parental } \\
\text { depression } \\
\text { Parental } \\
\text { functionin } \\
\mathrm{g} \\
\text { Parental } \\
\text { hostility }\end{array}$ & & $\begin{array}{l}\text { Pre disaster } \\
5 \text { months } \\
\text { post disaster }\end{array}$ & $\begin{array}{l}\text { Internalizi } \\
\text { ng } \\
\text { symptoms, } \\
\text { aggressive } \\
\text { behaviors } \\
\text { and fewer } \\
\text { prosocial } \\
\text { behaviors } \\
\text { in children }\end{array}$ \\
\hline $\begin{array}{l}\text { Thienkrua, } \\
\text { W., Cardozo, } \\
\text { B. L., } \\
\text { Chakkraband, } \\
\text { M. S., } \\
\text { Guadamuz, T. } \\
\text { E., Pengjuntr, } \\
\text { W., } \\
\text { Tantipiwatana } \\
\text { skul, P., ... \& } \\
\text { Tappero, J. } \\
\text { W. (2006). }\end{array}$ & $\begin{array}{l}\text { Tsunami } \\
\text { (Thailand) }\end{array}$ & $\begin{array}{l}\text { Longitudin } \\
\text { al }\end{array}$ & Individual & $\begin{array}{l}371 \text { children } \\
\text { aged } 7-14 \\
\text { years }\end{array}$ & $\begin{array}{l}\text { Living in } \\
\text { camps } \\
\text { displaced, } \\
\text { disaster } \\
\text { exposure, } \\
\text { age }\end{array}$ & & & $\begin{array}{l}2 \text { months } \\
\text { post disaster } \\
9 \text { months } \\
\text { post disaster }\end{array}$ & $\begin{array}{l}\text { PTSD } \\
\text { depression }\end{array}$ \\
\hline $\begin{array}{l}\text { Thomson, J., } \\
\text { Seers, K., } \\
\text { Frampton, C., } \\
\text { Hider, P., \& } \\
\text { Moor, S. } \\
(2016) .\end{array}$ & $\begin{array}{l}\text { Earthquake } \\
\text { (New } \\
\text { Zeeland) }\end{array}$ & $\begin{array}{l}\text { Longitudin } \\
\text { al }\end{array}$ & Individual & $\begin{array}{l}12014 \\
\text { children } \\
\text { aged 4-5 } \\
\text { years post- } \\
\text { disaster }\end{array}$ & $\begin{array}{l}\text { Time, } \\
\text { gender, } \\
\text { socio- } \\
\text { economic } \\
\text { status, } \\
\text { exposure }\end{array}$ & & & $\begin{array}{l}\text { Pre disaster, } \\
\text { september } \\
2010 \\
\text { (T1) }\end{array}$ & $\begin{array}{l}\text { Behaviour } \\
\text { al and } \\
\text { emotional } \\
\text { problems }\end{array}$ \\
\hline
\end{tabular}




\section{RESILIENCE IN CHILDREN AFTER NATURAL DISASTERS}

\begin{tabular}{|c|c|c|c|c|c|c|c|}
\hline & & & & $\begin{array}{l}\text { Parents } \\
\text { Teachers }\end{array}$ & & $\begin{array}{l}\text { Between } \\
\text { september } \\
2010 \text { and } \\
\text { february } 2011 \\
\text { (T2) } \\
\text { february- } \\
\text { december } \\
2011 \\
\text { (T3) }\end{array}$ & \\
\hline $\begin{array}{l}\text { Tian W, Jia } \\
\text { Z, Duan } \\
\text { G, Liu W, Pan } \\
\text { X, Guo } \\
\text { Q, Chen } \\
\text { R, Zhang X. } \\
(2013) .\end{array}$ & $\begin{array}{l}\text { Earthquake } \\
\text { (China) }\end{array}$ & $\begin{array}{l}\text { Longitudin } \\
\text { al }\end{array}$ & Individual & $\begin{array}{l}596 \text { children } \\
\text { and } \\
\text { adolescent } \\
\text { aged } 8-16 \\
\text { years }\end{array}$ & $\begin{array}{l}\text { Socio- } \\
\text { demographic } \\
\text { s, disaster } \\
\text { exposure } \\
\text { and } \\
\text { experience, } \\
\text { mental } \\
\text { health status }\end{array}$ & $\begin{array}{l}15 \text { months } \\
\text { post disaster } \\
36 \text { months } \\
\text { post disaster }\end{array}$ & $\begin{array}{l}\text { Health- } \\
\text { related } \\
\text { quality of } \\
\text { life }\end{array}$ \\
\hline $\begin{array}{l}\text { Uemoto, M., } \\
\text { Asakawa, A., } \\
\text { Takamiya, S., } \\
\text { Asakawa, K., } \\
\text { \& Inui, A. } \\
(2012) .\end{array}$ & $\begin{array}{l}\text { Earthquake } \\
\text { (Japan) }\end{array}$ & $\begin{array}{l}\text { Longitudin } \\
\text { al }\end{array}$ & Individual & $\begin{array}{l}8800 \text { school } \\
\text { children } \\
\text { exposed and } \\
\text { control } \\
\text { subjects } \\
\text { were } 1886 \\
\text { control } \\
\text { group } \\
3^{\text {rd }}, 5^{\text {th }} \text {, or } 8^{\text {th }} \\
\text { grade }\end{array}$ & $\begin{array}{l}\text { Disaster } \\
\text { exposure, } \\
\text { grade, } \\
\text { gender, } \\
\text { time }\end{array}$ & $\begin{array}{l}3 \text { and } 6 \\
\text { months post } \\
\text { disaster } \\
(\mathrm{T} 1-\mathrm{T} 2) \\
1 \text { and } 2 \text { years } \\
\text { Post disaster } \\
\text { (T3-T4) }\end{array}$ & PTSD \\
\hline $\begin{array}{l}\text { Usami, M., } \\
\text { Iwadare, Y., } \\
\text { Kodaira, M., } \\
\text { Watanabe, K., } \\
\text { Aoki, M., } \\
\text { Katsumi, C., } \\
\text {... \& Tanaka, } \\
\text { H. (2012). }\end{array}$ & $\begin{array}{l}\text { Earthquake } \\
\text { and } \\
\text { Tsunami } \\
\text { (Japan) }\end{array}$ & $\begin{array}{l}\text { Cross- } \\
\text { sectional }\end{array}$ & Individual & $\begin{array}{l}12524 \\
\text { children in } \\
\text { kindergarten } \\
\text { s, } \\
\text { elementary } \\
\text { schools and } \\
\text { junior high } \\
\text { schools in }\end{array}$ & $\begin{array}{l}\begin{array}{l}\text { Disaster } \\
\text { exposure, } \\
\text { bereavement }\end{array} \\
\text { 'experience } \\
\text { and life } \\
\text { in } \\
\text { evacuation } \\
\text { centers, } \\
\text { gender }\end{array}$ & $\begin{array}{l}8 \text { months } \\
\text { post disaster }\end{array}$ & PTSS \\
\hline
\end{tabular}




\begin{tabular}{|c|c|c|c|c|c|c|c|}
\hline $\begin{array}{l}\text { Usami, M., } \\
\text { Iwadare, Y., } \\
\text { Ushijima, H., } \\
\text { Inazaki, K., } \\
\text { Tanaka, T., } \\
\text { Kodaira, M., } \\
\text {.. Saito, K. } \\
\text { (2019) }\end{array}$ & $\begin{array}{l}\text { Earthquake } \\
\text { (Japan) }\end{array}$ & $\begin{array}{l}\text { Cross- } \\
\text { sectional }\end{array}$ & Invividual & $\begin{array}{l}262 \text { children, } \\
1-4 \text { years old }\end{array}$ & $\begin{array}{l}\text { Gender, age, } \\
\text { damage to } \\
\text { environment } \\
\text { al conditions } \\
\text { (house } \\
\text { damage, } \\
\text { evacuation } \\
\text { conditions, } \\
\text { and } \\
\text { bereavement } \\
\text { experience) }\end{array}$ & $\begin{array}{l}8,20,30, \text { and } \\
42 \text { months } \\
\text { after } \\
\text { earthquake }\end{array}$ & PTSS \\
\hline $\begin{array}{l}\text { Usami, M., } \\
\text { Iwadare, Y., } \\
\text { Watanabe, K., } \\
\text { Kodaira, M., } \\
\text { Ushijima, H., } \\
\text { Tanaka, T., ... } \\
\text { \& Saito, K. } \\
\text { (2014). }\end{array}$ & $\begin{array}{l}\text { Earthquake } \\
\text { and } \\
\text { Tsunami } \\
\text { (Japan) }\end{array}$ & $\begin{array}{l}\text { Longitudin } \\
\text { al }\end{array}$ & Individual & $\begin{array}{l}11639 \\
\text { children } \\
\text { aged } 10 \\
\text { years }\end{array}$ & $\begin{array}{l}\text { Time from } \\
\text { disaster }\end{array}$ & $\begin{array}{l}8 \text { months } \\
\text { post disaster } \\
(\mathrm{T} 1) \\
20 \text { months } \\
\text { post disaster } \\
30 \text { months } \\
\text { post disaster }\end{array}$ & $\begin{array}{l}\text { PTSS } \\
\text { PTSD } \\
\text { Depression }\end{array}$ \\
\hline $\begin{array}{l}\text { Valenti, M., } \\
\text { Ciprietti, T., } \\
\text { Di Egidio, C., } \\
\text { Gabrielli, M., } \\
\text { Masedu, F., } \\
\text { Tomassini, A. } \\
\text { R., \& Sorge, } \\
\text { G. (2012). }\end{array}$ & $\begin{array}{l}\text { Earthquake } \\
\text { (Italy) }\end{array}$ & $\begin{array}{l}\text { Pre-post } \\
\text { and } \\
\text { Longitudin } \\
\text { al }\end{array}$ & Individual & $\begin{array}{l}18 \\
\text { participants } \\
\text { with ASD } \\
\text { exposed } \\
42 \\
\text { participants } \\
\text { with ASD } \\
\text { no exposed }\end{array}$ & $\begin{array}{l}\text { Exposed and } \\
\text { non-exposed } \\
\text { participants, } \\
\text { age, } \\
\text { exposure } \\
\text { effect, } \\
\text { time effect }\end{array}$ & $\begin{array}{l}\text { A few days } \\
\text { before the } \\
\text { disaster } \\
6 \text { months } \\
\text { post disaster } \\
12 \text { months } \\
\text { post disaster }\end{array}$ & $\begin{array}{l}\text { Adaptive } \\
\text { behaviour } \\
\text { of } \\
\text { participant } \\
\text { S with } \\
\text { ASD }\end{array}$ \\
\hline $\begin{array}{l}\text { Vernberg, E. } \\
\text { M., La Greca, } \\
\text { A. M., } \\
\text { Silverman, W. } \\
\text { K., \& } \\
\text { Prinstein, M. } \\
\text { J. } \\
(1996) .\end{array}$ & $\begin{array}{l}\text { Hurricane } \\
\text { (USA) }\end{array}$ & $\begin{array}{l}\text { Cross- } \\
\text { Sectional }\end{array}$ & $\begin{array}{l}\text { Individual } \\
\text { and } \\
\text { Interpersona } \\
1\end{array}$ & $\begin{array}{l}568 \\
\text { Elementary, } \\
3^{\text {rd }}, 4^{\text {th }} \text { and } \\
5^{\text {th }} \text { grade }\end{array}$ & $\begin{array}{l}\text { Exposure to } \\
\text { traumatic } \\
\text { events, } \\
\quad \text { child } \\
\text { characteristi } \\
\text { cs, } \\
\text { access to } \\
\text { social } \\
\text { support, } \\
\text { children's } \\
\text { coping }\end{array}$ & $\begin{array}{l}3 \text { months } \\
\text { post disaster }\end{array}$ & PTSD \\
\hline
\end{tabular}




\section{RESILIENCE IN CHILDREN AFTER NATURAL DISASTERS}

\begin{tabular}{|c|c|c|c|c|c|c|c|c|c|}
\hline $\begin{array}{l}\text { Vezzali, L., } \\
\text { Cadamuro, } \\
\text { A., Versari, } \\
\text { A., } \\
\text { Giovannini, } \\
\text { D., \& } \\
\text { Trifiletti, E. } \\
(2015) \text {. }\end{array}$ & $\begin{array}{l}\text { Earthquake } \\
\text { (Italy) }\end{array}$ & $\begin{array}{l}\text { Cross- } \\
\text { Sectional }\end{array}$ & $\begin{array}{l}\text { Individual } \\
\text { and } \\
\text { Intergroup }\end{array}$ & $\begin{array}{l}517 \text { children } \\
\text { aged } 7-12 \\
\text { years }\end{array}$ & $\begin{array}{l}\text { Perceived } \\
\text { disaster } \\
\text { threat } \\
\text { exposure }\end{array}$ & 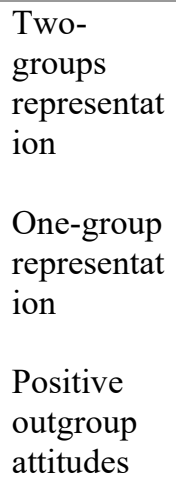 & $\begin{array}{l}\text { Participant's } \\
\text { group } \\
\text { of belonging }\end{array}$ & $\begin{array}{l}6 \text { months } \\
\text { post disaster }\end{array}$ & $\begin{array}{l}\text { Contact } \\
\text { behavioura } \\
1 \\
\text { intentions } \\
\text { Helping } \\
\text { behavioura } \\
1 \\
\text { intentions }\end{array}$ \\
\hline $\begin{array}{l}\text { Vezzali, L., } \\
\text { Drury, J., } \\
\text { Versari, A., \& } \\
\text { Cadamuro, A. } \\
\text { (2016). }\end{array}$ & $\begin{array}{l}\text { Earthquake } \\
\text { (Italy) }\end{array}$ & $\begin{array}{l}\text { Cross- } \\
\text { Sectional }\end{array}$ & $\begin{array}{l}\text { Individual } \\
\text { and } \\
\text { Interpersona } \\
1 \text { and Group }\end{array}$ & $\begin{array}{l}517 \text { children } \\
\text { aged } 7-12 \\
\text { years }\end{array}$ & PTSS & $\begin{array}{l}\text { Inclusion } \\
\text { Of The } \\
\text { Other In } \\
\text { The Self } \\
\text { One-Group } \\
\text { Representa } \\
\text { tion }\end{array}$ & & $\begin{array}{l}6 \text { months } \\
\text { post disaster }\end{array}$ & $\begin{array}{l}\text { Contact } \\
\text { intentions } \\
\text { Helping } \\
\text { intentions }\end{array}$ \\
\hline $\begin{array}{l}\text { Vigil, J.M., } \\
\text { Geary, D.C., } \\
\text { Granger, } \\
\text { D.A., } \\
\text { Flinn, M.V. } \\
(2010) .\end{array}$ & Hurricane & $\begin{array}{l}\text { Experiment } \\
\text { al study } \\
\text { (with } \\
\text { control } \\
\text { group) }\end{array}$ & Individual & $\begin{array}{l}62 \\
\text { adolescents } \\
\text { aged 12-19 } \\
\text { years } \\
52 \\
\text { adolescents } \\
\text { (control } \\
\text { group) }\end{array}$ & $\begin{array}{l}\text { Levels of } \\
\text { cortisol, } \\
\text { gender, } \\
\text { exposed vs } \\
\text { not exposed }\end{array}$ & & SNS activity & $\begin{array}{l}2 \text { months } \\
\text { post disaster }\end{array}$ & $\begin{array}{l}\text { Psychologi } \\
\text { cal } \\
\text { functionin } \\
\mathrm{g} \\
\text { hypothala } \\
\text { mic- } \\
\text { pituitary- } \\
\text { adrenal } \\
\text { activity }\end{array}$ \\
\hline $\begin{array}{l}\text { Vijayakumar, } \\
\text { L., Kannan, } \\
\text { G. K., \& } \\
\text { Daniel, S. J. } \\
\text { (2006). }\end{array}$ & $\begin{array}{l}\text { Tsunami } \\
\text { (Southeast } \\
\text { Asia) }\end{array}$ & $\begin{array}{l}\text { Cross- } \\
\text { Sectional }\end{array}$ & $\begin{array}{l}\text { Individual } \\
\text { and } \\
\text { Interpersona } \\
1\end{array}$ & $\begin{array}{l}230 \text { children } \\
\text { aged 11-14 } \\
\text { years }\end{array}$ & $\begin{array}{l}\text { Disaster } \\
\text { exposure, } \\
\text { tsunami- } \\
\text { related } \\
\text { factors } \\
\text { demographic } \\
\text { s, }\end{array}$ & & & $\begin{array}{l}12 \text { months } \\
\text { post disaster }\end{array}$ & PTSD \\
\hline
\end{tabular}




\begin{tabular}{|c|c|c|c|c|c|c|c|}
\hline & & & & & $\begin{array}{l}\text { pre-existing } \\
\text { psychopatho } \\
\text { logy of the } \\
\text { children, } \\
\text { family } \\
\text { mental } \\
\text { health }\end{array}$ & & \\
\hline $\begin{array}{l}\text { Wang, M., } \\
\text { Armour, C., } \\
\text { Li, X., Dai, } \\
\text { X., Zhu, X., \& } \\
\text { Yao, S. } \\
\text { (2013). }\end{array}$ & $\begin{array}{l}\text { Earthquake } \\
\text { (China) }\end{array}$ & $\begin{array}{l}\text { Cross- } \\
\text { Sectional }\end{array}$ & Individual & $\begin{array}{l}571 \\
\text { adolescent } \\
\text { aged 12-17 } \\
\text { years }\end{array}$ & Gender & & PTSD \\
\hline $\begin{array}{l}\text { Wang, Wei; } \\
\text { Fu, Wei; Wu, } \\
\text { Jin; Ma, Xian- } \\
\text { cang; Sun, } \\
\text { Xue-li; } \\
\text { Huang, Yi; } \\
\text { Hashimoto, } \\
\text { Kenji; Gao, } \\
\text { Cheng-ge; } \\
\text { (2012). }\end{array}$ & $\begin{array}{l}\text { Earthquake } \\
\text { (China) }\end{array}$ & $\begin{array}{l}\text { Cross- } \\
\text { Sectional }\end{array}$ & Individual & $\begin{array}{l}1841 \\
\text { students } \\
\text { aged 11-20 } \\
\text { years }\end{array}$ & $\begin{array}{l}\text { Age, gender, } \\
\text { grade, } \\
\text { disaster } \\
\text { exposure, } \\
\text { impact of } \\
\text { event, } \\
\text { personal } \\
\text { experiences } \\
\text { during the } \\
\text { earthquake, } \\
\text { living } \\
\text { environment }\end{array}$ & $\begin{array}{l}10 \text { months } \\
\text { post disaster }\end{array}$ & $\begin{array}{l}\text { PTSD } \\
\text { Depression }\end{array}$ \\
\hline $\begin{array}{l}\text { Ward, M. E., } \\
\text { Shelley, K., } \\
\text { Kaase, K., \& } \\
\text { Pane, J. F. } \\
(2008) .\end{array}$ & $\begin{array}{l}\text { Hurricane } \\
\text { (USA) }\end{array}$ & $\begin{array}{l}\text { Cross- } \\
\text { Sectional }\end{array}$ & Individual & $\begin{array}{l}\text { Pre- } \\
\text { kindergarten } \\
\text { to } 12^{\text {th }} \text { grade } \\
\text { students }\end{array}$ & $\begin{array}{l}\text { Demographi } \\
\text { c } \\
\text { characteristi } \\
\text { cs, } \\
\text { displaced vs } \\
\text { no displaced }\end{array}$ & $\begin{array}{l}\text { in the first two } \\
\text { years } \\
\text { post disaster }\end{array}$ & $\begin{array}{l}\text { Achieveme } \\
\text { nt and } \\
\text { behavior }\end{array}$ \\
\hline $\begin{array}{l}\text { Wasserstein, } \\
\text { S. B., \& La } \\
\text { Greca, A. M. } \\
\text { (1998). }\end{array}$ & $\begin{array}{l}\text { Hurricane } \\
\text { (USA) }\end{array}$ & $\begin{array}{l}\text { Cross- } \\
\text { Sectional }\end{array}$ & $\begin{array}{l}\text { Individual, } \\
\text { Interpersona } \\
1 \text { and } \\
\text { Intergroup }\end{array}$ & $\begin{array}{l}89 \\
\text { elementary } \\
\text { School } \\
\text { children } 4^{\text {th }} \\
\text { to } 6^{\text {th }} \text { grades }\end{array}$ & $\begin{array}{l}\text { Gender, } \\
\text { ethnicity, } \\
\text { perceived } \\
\text { parental } \\
\text { conflict, } \\
\text { anxiety } \\
\text { level, }\end{array}$ & $\begin{array}{l}3 \text { months } \\
\text { post disaster }\end{array}$ & $\begin{array}{l}\text { PTSD } \\
\text { Anxiety }\end{array}$ \\
\hline
\end{tabular}




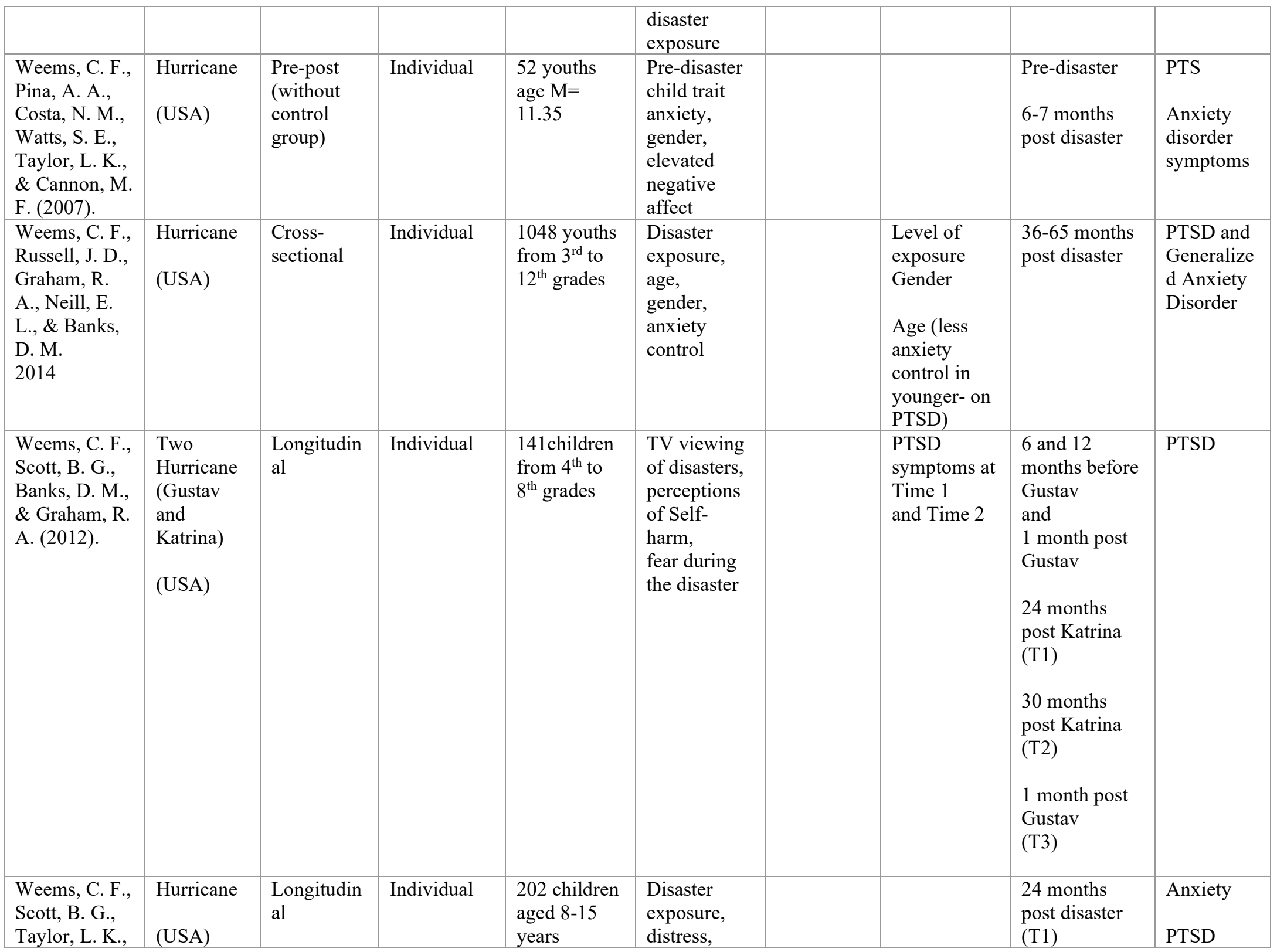




\section{RESILIENCE IN CHILDREN AFTER NATURAL DISASTERS}

\begin{tabular}{|c|c|c|c|c|c|c|c|c|}
\hline $\begin{array}{l}\text { Cannon, M. } \\
\text { F., Romano, } \\
\text { D. M., \& } \\
\text { Perry, A. M. } \\
(2013) .\end{array}$ & & & & & $\begin{array}{l}\text { PTSD } \\
\text { symptoms, } \\
\text { anxiety, } \\
\text { time }\end{array}$ & & $\begin{array}{l}30 \text { months } \\
\text { post disaster } \\
\text { (T2) }\end{array}$ & $\begin{array}{l}\text { Achieveme } \\
\text { nt }\end{array}$ \\
\hline $\begin{array}{l}\text { Weems, C. F., } \\
\text { Taylor, L. K., } \\
\text { Cannon, M. } \\
\text { F., Marino, R. } \\
\text { C., Romano, } \\
\text { D. M., Scott, } \\
\text { B. G., ... \& } \\
\text { Triplett, V. } \\
(2010) .\end{array}$ & $\begin{array}{l}\text { Hurricane } \\
\text { (USA) }\end{array}$ & $\begin{array}{l}\text { Longitudin } \\
\text { al }\end{array}$ & Individual & $\begin{array}{l}191 \text { african } \\
\text { american } \\
\text { aged } 8-15 \\
\text { years }\end{array}$ & $\begin{array}{l}\text { Age, } \\
\text { gender, } \\
\text { exposure to } \\
\text { traumatic } \\
\text { experiences, } \\
\text { time }\end{array}$ & & $\begin{array}{l}24 \text { Months } \\
\text { post disaster } \\
\text { (T1) } \\
30 \text { Months } \\
\text { Post disaster } \\
\text { (T2) }\end{array}$ & PTSD \\
\hline $\begin{array}{l}\text { Xie, Y., Wu, } \\
\text { J., \& Shen, G. } \\
(2019)\end{array}$ & $\begin{array}{l}\text { Earthquake } \\
\text { (Tibet) }\end{array}$ & $\begin{array}{l}\text { Cross- } \\
\text { sectional }\end{array}$ & Indivudual & $\begin{array}{l}850 \text { children } \\
11 \text { - } 20 \text { years } \\
\text { old }\end{array}$ & $\begin{array}{l}\text { Age, gender, } \\
\text { grade, } \\
\text { ethnicity, } \\
\text { level of } \\
\text { exposure, } \\
\text { PTSD, } \\
\text { depression }\end{array}$ & & $\begin{array}{l}6 \text { years after } \\
\text { the earthquake }\end{array}$ & $\begin{array}{l}\text { posttrauma } \\
\text { tic growth } \\
\text { (PTG) and } \\
\text { posttrauma } \\
\text { tic stress } \\
\text { disorder } \\
\text { (PTSD) }\end{array}$ \\
\hline $\begin{array}{l}\text { Xu, J., Xie, } \\
\text { L., Li, B., Li, } \\
\text { N., \& Yang, } \\
\text { Y. } \\
(2012) .\end{array}$ & $\begin{array}{l}\text { Earthquake } \\
\text { (China) }\end{array}$ & $\begin{array}{l}\text { Cross- } \\
\text { Sectional }\end{array}$ & Individual & $\begin{array}{l}21652 \\
\text { children } \\
\text { aged } 7-15 \\
\text { years }\end{array}$ & $\begin{array}{l}\text { Gender, } \\
\text { age, } \\
\text { cultural } \\
\text { differences, } \\
\text { disaster } \\
\text { exposure }\end{array}$ & & $\begin{array}{l}12 \text { months } \\
\text { post disaster }\end{array}$ & $\begin{array}{l}\text { Anxiety } \\
\text { symptoms }\end{array}$ \\
\hline $\begin{array}{l}\text { Yagi, J., } \\
\text { Fujiwara, T., } \\
\text { Yambe, T., } \\
\text { Okuyama, M., } \\
\text { Kawachi, I., } \\
\text { \& Sakai, A. } \\
\text { (2016). }\end{array}$ & $\begin{array}{l}\text { Earthquake } \\
\text { (Japan) }\end{array}$ & $\begin{array}{l}\text { Cross - } \\
\text { Sectional }\end{array}$ & $\begin{array}{l}\text { Individual } \\
\text { and } \\
\text { Interpersona } \\
1\end{array}$ & $\begin{array}{l}94 \text { children, } \\
\text { aged } 5-7 \\
\text { years }\end{array}$ & $\begin{array}{l}\text { Social } \\
\text { capital, } \\
\text { traumatic } \\
\text { events, age }\end{array}$ & $\begin{array}{l}\text { Care givers } \\
\text { mental } \\
\text { health, } \\
\text { Child } \\
\text { PTSD }\end{array}$ & $\begin{array}{l}2 \text { years } \\
\text { post disaster }\end{array}$ & $\begin{array}{l}\text { Behavior } \\
\text { problems }\end{array}$ \\
\hline $\begin{array}{l}\text { Yang, R., } \\
\text { Xiang, Y. T., } \\
\text { Shuai, L., } \\
\text { Qian, Y., Lai, } \\
\text { K. Y., } \\
\text { Ungvari, G. }\end{array}$ & $\begin{array}{l}\text { Earthquake } \\
\text { (China) }\end{array}$ & $\begin{array}{l}\text { Longitudin } \\
\text { al }\end{array}$ & Individual & $\begin{array}{l}100 \text { children } \\
\text { Aged 9-13 } \\
\text { years } \\
\text { and } \\
14-17 \text { years }\end{array}$ & $\begin{array}{l}\text { PTSD group } \\
\text { ( } 34 \\
\text { adopted) } \\
\text { vs no PTSD } \\
\text { group } \\
\text { ( } 66 \text { orphan) }\end{array}$ & & $\begin{array}{l}4 \text { months } \\
\text { post disaster } \\
\text { (T1) } \\
12 \text { months } \\
\text { post disaster }\end{array}$ & $\begin{array}{l}\text { Executive } \\
\text { functions }\end{array}$ \\
\hline
\end{tabular}




\begin{tabular}{|c|c|c|c|c|c|c|c|c|}
\hline $\begin{array}{l}\text { S., ...\& } \\
\text { Wang, Y. F. } \\
\text { (2014). }\end{array}$ & & & & & exposure & & (T2) & \\
\hline $\begin{array}{l}\text { Yeung, N. C., } \\
\text { Lau, J. T., Yu, } \\
\text { N. X., Zhang, } \\
\text { J., Xu, Z., } \\
\text { Choi, K. C., ... } \\
\text { \& Lui, W. W. } \\
\text { (2016). }\end{array}$ & $\begin{array}{l}\text { Earthquake } \\
\text { (China) }\end{array}$ & $\begin{array}{l}\text { Longitudin } \\
\text { al }\end{array}$ & Individual & $\begin{array}{l}3577 \\
\text { students } 5^{\text {th }}- \\
12^{\text {th }} \text { grade }\end{array}$ & $\begin{array}{l}\text { Exposure to } \\
\text { earthquake- } \\
\text { related } \\
\text { imagery, } \\
\text { content, } \\
\text { perceptions } \\
\text { and } \\
\text { emotional } \\
\text { reactions } \\
\text { related to the } \\
\text { disaster, } \\
\text { prior } \\
\text { traumatic } \\
\text { experience, } \\
\text { gender, } \\
\text { age }\end{array}$ & & $\begin{array}{l}1 \text { month } \\
\text { post disaster } \\
\text { (T1) } \\
6 \text { months } \\
\text { after the } \mathrm{T} 1 \\
\text { (T2) }\end{array}$ & PTSD \\
\hline $\begin{array}{l}\text { Ying, L. H., } \\
\text { Wu, X. C., \& } \\
\text { Chen, C. } \\
\text { (2013). }\end{array}$ & $\begin{array}{l}\text { Earthquake } \\
\text { (China) }\end{array}$ & $\begin{array}{l}\text { Cross- } \\
\text { Sectional }\end{array}$ & Individual & $\begin{array}{l}3052 \\
\text { children } \\
\text { aged 8-19 } \\
\text { years }\end{array}$ & $\begin{array}{l}\text { Age, } \\
\text { gender, } \\
\text { post-trauma } \\
\text { experience, } \\
\text { severity of } \\
\text { disaster } \\
\text { exposure }\end{array}$ & & $\begin{array}{l}1 \text { year } \\
\text { post disaster }\end{array}$ & $\begin{array}{l}\text { PTSD and } \\
\text { depression }\end{array}$ \\
\hline $\begin{array}{l}\text { Ying, L., Wu, } \\
\text { X., Lin, C., \& } \\
\text { Jiang, L. } \\
\text { (2014). }\end{array}$ & $\begin{array}{l}\text { Earthquake } \\
\text { (China) }\end{array}$ & $\begin{array}{l}\text { Cross- } \\
\text { Sectional }\end{array}$ & Individual & $\begin{array}{l}788 \\
\text { adolescent } \\
\text { aged 12-19 } \\
\text { years }\end{array}$ & $\begin{array}{l}\text { Severity of } \\
\text { disaster } \\
\text { exposure, } \\
\text { trait } \\
\text { resilience, } \\
\text { age, } \\
\text { gender }\end{array}$ & Resilience & $\begin{array}{l}12 \text { and } 18 \\
\text { months } \\
\text { post disaster } \\
\text { (T1-T2) } \\
24 \text { and } 30 \\
\text { months } \\
\text { Post disaster } \\
\text { (T3-T4) }\end{array}$ & $\begin{array}{l}\text { PTSD } \\
\text { depressive } \\
\text { symptoms }\end{array}$ \\
\hline $\begin{array}{l}\text { Yorbik, O., } \\
\text { Akbiyik, D. I., } \\
\text { Kirmizigul, } \\
\text { P., \& }\end{array}$ & $\begin{array}{l}\text { Earthquake } \\
\text { (Turkey) }\end{array}$ & $\begin{array}{l}\text { Cross- } \\
\text { Sectional }\end{array}$ & Individual & $\begin{array}{l}35 \text { children } \\
\text { aged } 2-16 \\
\text { years }\end{array}$ & Age & & & PTSD \\
\hline
\end{tabular}




\section{RESILIENCE IN CHILDREN AFTER NATURAL DISASTERS}

\begin{tabular}{|c|c|c|c|c|c|c|c|}
\hline $\begin{array}{l}\text { Söhmen, T. } \\
\text { (2004). }\end{array}$ & & & & & & & \\
\hline $\begin{array}{l}\text { Yoshida, H., } \\
\text { Kobayashi, } \\
\text { N., Honda, } \\
\text { N., Matsuoka, } \\
\text { H., } \\
\text { Yamaguchi, } \\
\text { T., Homma, } \\
\text { H., \& Tomita, } \\
\text { H. (2016). }\end{array}$ & $\begin{array}{l}\text { Earthquake } \\
\text { (Japan) }\end{array}$ & $\begin{array}{l}\text { Cross- } \\
\text { Sectional }\end{array}$ & Individual & $\begin{array}{l}3337 \\
\text { children } \\
\text { aged 9-15 } \\
\text { years } \\
\text { Parents } \\
\text { Teachers }\end{array}$ & $\begin{array}{l}\text { Experience } \\
\text { of the } \\
\text { disaster, } \\
\text { prior } \\
\text { traumatic } \\
\text { experience, } \\
\text { children's } \\
\text { attitude } \\
\text { toward } \\
\text { memories of } \\
\text { the disaster } \\
\text { (rumination) } \\
\text { gender, } \\
\text { grade }\end{array}$ & $\begin{array}{l}31 \text { months } \\
\text { post disaster }\end{array}$ & $\begin{array}{l}\text { Posttrauma } \\
\text { tic growth } \\
\text { Children } \\
\text { PTSS }\end{array}$ \\
\hline $\begin{array}{l}\text { Zhang, J., } \\
\text { Zhu, S., Du, } \\
\text { C., \& Zhang, } \\
\text { Y. (2015). }\end{array}$ & $\begin{array}{l}\text { Earthquake } \\
\text { (China) }\end{array}$ & $\begin{array}{l}\text { Longitudin } \\
\text { al }\end{array}$ & Individual & $\begin{array}{l}2299 \\
\text { children } \\
\text { aged 8-19 } \\
\text { years }\end{array}$ & $\begin{array}{l}\text { Age, } \\
\text { ethnicity, } \\
\text { gender, } \\
\text { PTSD, } \\
\text { symptoms, } \\
\text { time }\end{array}$ & $\begin{array}{l}3 \text { months } \\
\text { post disaster } \\
\text { (T1) } \\
6 \text { months } \\
\text { Post disaster } \\
\text { (T2) }\end{array}$ & $\begin{array}{l}\text { PTSD } \\
\text { somatic } \\
\text { symptoms }\end{array}$ \\
\hline $\begin{array}{l}\text { Zhang, W., } \\
\text { Rajendran, K., } \\
\text { Ham, J., } \\
\text { Finik, J., } \\
\text { Buthmann, J., } \\
\text { Davey, K., ... } \\
\text { \& Nomura, Y. } \\
\text { (2018). }\end{array}$ & $\begin{array}{l}\text { Hurricane } \\
\text { (USA) }\end{array}$ & $\begin{array}{l}\text { Longitudin } \\
\text { al }\end{array}$ & $\begin{array}{l}\text { Individual } \\
\text { and } \\
\text { Interpersona } \\
1\end{array}$ & $\begin{array}{l}318 \text { mother- } \\
\text { child dyads }\end{array}$ & $\begin{array}{l}\text { Objective } \\
\text { exposure, } \\
\text { subjective } \\
\text { stress } \\
\text { reaction }\end{array}$ & $\begin{array}{l}6 \text { and } 12 \\
\text { months } \\
\text { post disaster } \\
(\mathrm{T} 1-\mathrm{T} 2) \\
18 \text { and } 24 \\
\text { months } \\
\text { post disaster } \\
(\mathrm{T} 3-\mathrm{T} 4)\end{array}$ & $\begin{array}{l}\text { Developm } \\
\text { ental } \\
\text { trajectory } \\
\text { of } \\
\text { temperame } \\
\text { nt }\end{array}$ \\
\hline $\begin{array}{l}\text { Zhang, X., } \\
\text { Liu, M., Zhu, } \\
\text { M., Shi, J., \& } \\
\text { Cheng, L. } \\
(2010) .\end{array}$ & $\begin{array}{l}\text { Earthquake } \\
\text { (China) }\end{array}$ & $\begin{array}{l}\text { Cross- } \\
\text { Sectional }\end{array}$ & Individual & $\begin{array}{l}196 \text { pre } \\
\text { disaster and } \\
116 \text { post } \\
\text { disaster } \\
\text { orphans }\end{array}$ & $\begin{array}{l}\text { Personality, } \\
\text { impact of } \\
\text { event }\end{array}$ & $\begin{array}{l}6 \text { months } \\
\text { post disaster }\end{array}$ & PTSD \\
\hline
\end{tabular}




\section{RESILIENCE IN CHILDREN AFTER NATURAL DISASTERS}

\begin{tabular}{|c|c|c|c|c|c|c|c|}
\hline & & & & $\begin{array}{l}\text { aged } 8-18 \\
\text { years }\end{array}$ & & & \\
\hline $\begin{array}{l}\text { Zhang, Y., } \\
\text { Kong, F., } \\
\text { Wang, L., } \\
\text { Chen, H., } \\
\text { Gao, X., Tan, } \\
\text { X., ... \& Liu, } \\
\text { Y. (2010). }\end{array}$ & $\begin{array}{l}\text { Earthquake } \\
\text { (China) }\end{array}$ & $\begin{array}{l}\text { Cross- } \\
\text { Sectional }\end{array}$ & $\begin{array}{l}\text { Individual } \\
\text { and } \\
\text { Interpersona } \\
1\end{array}$ & $\begin{array}{l}423 \text { children } \\
\text { and } \\
\text { adolescents, } \\
\text { aged } 11-16 \\
\text { years }\end{array}$ & $\begin{array}{l}\text { Highly } \\
\text { exposed area } \\
\text { and a lowly } \\
\text { exposed } \\
\text { area, } \\
\text { disaster } \\
\text { situation, } \\
\text { exposure } \\
\text { degree, } \\
\text { family } \\
\text { situation, } \\
\text { times of } \\
\text { experiencing } \\
\text { other } \\
\text { stressful } \\
\text { events }\end{array}$ & $\begin{array}{l}12 \text { months } \\
\text { post disaster }\end{array}$ & $\begin{array}{l}\text { Post- } \\
\text { traumatic } \\
\text { psychologi } \\
\text { cal } \\
\text { reactions } \\
\text { Coping } \\
\text { styles }\end{array}$ \\
\hline $\begin{array}{l}\text { Zhang, Y., } \\
\text { Zhang, J., } \\
\text { Zhu, S., Du, } \\
\text { C., \& Zhang, } \\
\text { W. (2015). }\end{array}$ & $\begin{array}{l}\text { Earthquake } \\
\text { (China) }\end{array}$ & $\begin{array}{l}\text { Cross- } \\
\text { Sectional }\end{array}$ & Individual & $\begin{array}{l}3053 \\
\text { children } \\
\text { aged 8-19 } \\
\text { years }\end{array}$ & $\begin{array}{l}\text { Age, } \\
\text { ethnicity, } \\
\text { gender, } \\
\text { disaster } \\
\text { exposure, } \\
\text { impact of } \\
\text { event, } \\
\text { PTSD }\end{array}$ & $\begin{array}{l}3 \text { months } \\
\text { post disaster }\end{array}$ & $\begin{array}{l}\text { Somatic } \\
\text { symptoms }\end{array}$ \\
\hline $\begin{array}{l}\text { Zhou, P., } \\
\text { Zhang, Y., } \\
\text { Wei, C., Liu, } \\
\text { Z., \& Hannak, } \\
\text { W. } \\
(2016) .\end{array}$ & $\begin{array}{l}\text { Earthquake } \\
\text { (China) }\end{array}$ & $\begin{array}{l}\text { Longitudin } \\
\text { al }\end{array}$ & Individual & $\begin{array}{l}197 \text { Students } \\
\text { from } 4^{\text {th }} \text { to } \\
8^{\text {th }} \text { grade }\end{array}$ & $\begin{array}{l}\text { Acute stress } \\
\text { disorder, } \\
\text { disaster } \\
\text { exposure }\end{array}$ & $\begin{array}{l}2 \text { months } \\
\text { post disaster } \\
\text { (T1) } \\
6 \text { months } \\
\text { post disaster } \\
\text { (T2) } \\
12 \text { months } \\
\text { post disaster } \\
\text { (T3) }\end{array}$ & PTSD \\
\hline
\end{tabular}




\begin{tabular}{|c|c|c|c|c|c|c|c|c|}
\hline $\begin{array}{l}\text { Zhou, X., \& } \\
\text { Wu, X. } \\
(2016) .\end{array}$ & $\begin{array}{l}\text { Earthquake } \\
\text { (China) }\end{array}$ & $\begin{array}{l}\text { Longitudin } \\
\text { al }\end{array}$ & Individual & $\begin{array}{l}310 \\
\text { adolescents } \\
\text { aged 12-19 } \\
\text { years }\end{array}$ & $\begin{array}{l}\text { Event- } \\
\text { related } \\
\text { rumination, } \\
\text { time }\end{array}$ & $\begin{array}{l}\text { Intrusive } \\
\text { and } \\
\text { deliberate } \\
\text { rumination } \\
\text { (At T2) }\end{array}$ & $\begin{array}{l}6 \text { months } \\
\text { post disaster } \\
\text { (T1) } \\
12 \text { months } \\
\text { post disaster } \\
\text { (T2) } \\
18 \text { months } \\
\text { post disaster } \\
\text { (T3) }\end{array}$ & $\begin{array}{l}\text { Post- } \\
\text { traumatic } \\
\text { Growth } \\
\text { PTSD }\end{array}$ \\
\hline $\begin{array}{l}\text { Zhou, X., \& } \\
\text { Wu, X., } \\
\text { Wenchao, W., } \\
\text { Tian, Y. } \\
\text { (2017). }\end{array}$ & $\begin{array}{l}\text { Earthquake } \\
\text { (China) }\end{array}$ & $\begin{array}{l}\text { Longitudin } \\
\text { al }\end{array}$ & $\begin{array}{l}\text { Individual } \\
\text { and } \\
\text { Interpersona } \\
1\end{array}$ & $\begin{array}{l}303 \text { children } \\
12-19 \text { years }\end{array}$ & $\begin{array}{l}\text { Social } \\
\text { support, } \\
\text { PTSD, } \\
\text { posttraumati } \\
\text { c growth }\end{array}$ & & $\begin{array}{l}6 \text { months } \\
\text { post disaster } \\
\text { (T1) } \\
12 \text { months } \\
\text { post disaster } \\
\text { (T2) } \\
18 \text { months } \\
\text { post disaster } \\
\text { (T3) }\end{array}$ & $\begin{array}{l}\text { PTSD } \\
\text { Post- } \\
\text { traumatic } \\
\text { Growth }\end{array}$ \\
\hline $\begin{array}{l}\text { Zhou, X., Wu, } \\
\text { X., \& Zhen, } \\
\text { R. (2017). }\end{array}$ & $\begin{array}{l}\text { Earthquake } \\
\text { (China) }\end{array}$ & $\begin{array}{l}\text { Cross- } \\
\text { Sectional }\end{array}$ & $\begin{array}{l}\text { Individual } \\
\text { and } \\
\text { Interpersona } \\
1\end{array}$ & $\begin{array}{l}309 \\
\text { adolescents } \\
\text { aged 12-18 } \\
\text { years }\end{array}$ & $\begin{array}{l}\text { Disaster } \\
\text { exposure, } \\
\text { social } \\
\text { support, } \\
\text { emotion } \\
\text { regulation }\end{array}$ & $\begin{array}{l}\text { Cognitive } \\
\text { reappraisal } \\
\text { Expressive } \\
\text { suppressio } \\
\text { n. }\end{array}$ & $\begin{array}{l}6 \text { months } \\
\text { post disaster }\end{array}$ & $\begin{array}{l}\text { PTSD } \\
\text { Post- } \\
\text { traumatic } \\
\text { Growth }\end{array}$ \\
\hline $\begin{array}{l}\text { Zhou, X., Wu, } \\
\text { X., \& Zhen, } \\
\text { R. (2018) }\end{array}$ & $\begin{array}{l}\text { Earthquake } \\
\text { (China) }\end{array}$ & $\begin{array}{l}\text { Cross- } \\
\text { sectional }\end{array}$ & Individual & $\begin{array}{l}591 \text { children } \\
11-19 \text { years } \\
\text { old }\end{array}$ & $\begin{array}{l}\text { age, sex, } \\
\text { traumatic } \\
\text { exposure. }\end{array}$ & & $\begin{array}{l}1 \text { year after } \\
\text { the } \\
\text { earthquake }\end{array}$ & $\begin{array}{l}\text { Posttrauma } \\
\text { tic stress } \\
\text { disorder } \\
\text { (PTSD) } \\
\text { and } \\
\text { posttrauma } \\
\text { tic growth } \\
\text { (PTG) }\end{array}$ \\
\hline $\begin{array}{l}\text { Zhou, X., Wu, } \\
\text { X., Zhen, R., }\end{array}$ & $\begin{array}{l}\text { Earthquake } \\
\text { (China) }\end{array}$ & $\begin{array}{l}\text { Longitudin } \\
\text { al }\end{array}$ & Individual & $\begin{array}{l}391 \text { youth } \\
\text { aged 12-19 } \\
\text { years }\end{array}$ & $\begin{array}{l}\text { Disaster } \\
\text { exposure, } \\
\text { age }\end{array}$ & & $\begin{array}{l}12 \text { and } 18 \\
\text { months } \\
\text { post disaster }\end{array}$ & PTSD \\
\hline
\end{tabular}




\section{RESILIENCE IN CHILDREN AFTER NATURAL DISASTERS}

\begin{tabular}{|l|l|l|l|l|l|l|l|l|}
\hline $\begin{array}{l}\text { Wang, W., \& } \\
\text { Tian, Y. } \\
\text { (2018). }\end{array}$ & & & & gender & & & \\
& & & & & & & $\begin{array}{l}\text { (T1-T2) } \\
24 \text { and } 30 \\
\text { months } \\
\text { post disaster } \\
\text { (T3-T4) }\end{array}$ \\
\hline $\begin{array}{l}\text { Zhou, X., } \\
\text { Zhen, R., \& } \\
\text { Wu, X. (2017) }\end{array}$ & $\begin{array}{l}\text { Earthquake } \\
\text { (China) }\end{array}$ & $\begin{array}{l}\text { Cross- } \\
\text { sectional }\end{array}$ & Individual & $\begin{array}{l}736 \text { children } \\
12-19 \text { years } \\
\text { old }\end{array}$ & $\begin{array}{l}\text { PTSD and } \\
\text { control } \\
\text { beliefs } \\
\text { Gender } \\
\text { Age }\end{array}$ & & $\begin{array}{l}\text { primary } \\
\text { control year after } \\
\text { beliefs } \\
\text { the } \\
\text { earthquake }\end{array}$ & $\begin{array}{l}\text { Academic } \\
\text { burnout }\end{array}$ \\
\hline
\end{tabular}

Note. Although some studies have been classified as experimental, most of them have a quasi-experimental design, since assignment to conditions was not completely random but determined by exposure or not to a disaster. PTSD= Posttraumatic Stress Disorder; PTSS= Posttraumatic Stress Symptoms; PTS = Posttraumatic Symptoms; GAD= Generalized Anxiety Disorder; ASD= Autistic Spectrum Disorder 


\section{RESILIENCE IN CHILDREN AFTER NATURAL DISASTERS}

Table 2_OSM. Reviews and meta-analyses $(N=28)$

\begin{tabular}{|c|c|c|c|c|c|c|}
\hline Study & Disaster & Type & $\begin{array}{l}\text { Number of } \\
\text { studies } \\
\text { considered }\end{array}$ & Predictor(s) & Moderator(s) & Dependent Variable(s) \\
\hline $\begin{array}{l}\text { Brown, R. C., } \\
\text { Witt, A., Fegert, } \\
\text { J. M., Keller, F., } \\
\text { Rassenhofer, } \\
\text { M., \& Plener, P. } \\
\text { L. } \\
\text { (2017) }\end{array}$ & $\begin{array}{l}\text { Man-made and natural } \\
\text { disasters }\end{array}$ & $\begin{array}{l}\text { Meta-analysis } \\
\text { and systematic } \\
\text { review }\end{array}$ & 36 studies & & $\begin{array}{l}\text { Profession of } \\
\text { treatment providers } \\
\text { Treatment setting } \\
\text { (individual or } \\
\text { group) } \\
\text { Assessment } \\
\text { method of } \\
\text { PTSD }\end{array}$ & $\begin{array}{l}\text { Efficacy of Psychosocial } \\
\text { interventions for children, } \\
\text { (CBT, EMDR, } \\
\text { KIDNET and classroom- } \\
\text { based interventions) }\end{array}$ \\
\hline $\begin{array}{l}\text { Cartwright, C., } \\
\text { Hall, M., \& } \\
\text { Lee, A. C. K. } \\
(2017)\end{array}$ & Earthquake & Review & 152 studies & & & \\
\hline $\begin{array}{l}\text { Drury, S. S., } \\
\text { Scheeringa, M. } \\
\text { S., \& Zeanah, } \\
\text { C. H. } \\
(2008)\end{array}$ & Hurricane & Review & 7 studies & & & \\
\hline $\begin{array}{l}\text { Farooqui, M., } \\
\text { Quadri, S. A., } \\
\text { Suriya, S. S., } \\
\text { Khan, M. A., } \\
\text { Ovais, M., } \\
\text { Sohail, Z., et al. } \\
\text { (2017) }\end{array}$ & Earthquake & Review & 77 studies & & & \\
\hline $\begin{array}{l}\text { Forman- } \\
\text { Hoffman, V. L., } \\
\text { Zolotor, A. J., } \\
\text { McKeeman, J. }\end{array}$ & $\begin{array}{l}\text { Accidents, natural } \\
\text { disasters, } \\
\text { war }\end{array}$ & Review & 25 studies & & & \\
\hline
\end{tabular}




\section{RESILIENCE IN CHILDREN AFTER NATURAL DISASTERS}

\begin{tabular}{|c|c|c|c|c|c|c|}
\hline $\begin{array}{l}\text { L., Blanco, R., } \\
\text { Knauer, S. R., } \\
\text { Lloyd, S. W., \& } \\
\text { Viswanathan, } \\
\text { M. } \\
(2013)\end{array}$ & & & & & & \\
\hline $\begin{array}{l}\text { Fu, C., \& } \\
\text { Underwood, C. } \\
(2015)\end{array}$ & $\begin{array}{l}\text { Natural and/or man-made } \\
\text { disaster. }\end{array}$ & $\begin{array}{l}\text { Meta-analysis } \\
\text { and review }\end{array}$ & $\begin{array}{l}11 \text { studies } \\
\text { (4 from natural } \\
\text { disasters and } 7 \\
\text { from conflict- } \\
\text { affected areas) }\end{array}$ & & & $\begin{array}{l}\text { Effectiveness of school- } \\
\text { based mental-health and } \\
\text { psychosocial interventions } \\
\text { on PTSD }\end{array}$ \\
\hline $\begin{array}{l}\text { Gordon- } \\
\text { Hollingsworth, } \\
\text { A. T., Yao, N., } \\
\text { Chen, H., Qian, } \\
\text { M., \& Chen, S. } \\
(2015)\end{array}$ & Natural Disaster & Meta-analysis & 59 studies & $\begin{array}{l}\text { Age, gender, father } \\
\text { level of education, } \\
\text { urban versus rural } \\
\text { residence, experience } \\
\text { of prior trauma, } \\
\text { bereavement, having a } \\
\text { family } \\
\text { member injured, } \\
\text { knowing someone } \\
\text { other than a family } \\
\text { member injured or } \\
\text { killed, } \\
\text { witnessing someone } \\
\text { get injured or killed, } \\
\text { suffering personal } \\
\text { injury during the } \\
\text { trauma, becoming } \\
\text { trapped or buried, } \\
\text { house damage, loss of } \\
\text { property, trauma } \\
\text { severity, perceived } \\
\text { threat or fear during }\end{array}$ & $\begin{array}{l}\text { Type of disaster, } \\
\text { first evaluative } \\
\text { time-point, disaster } \\
\text { severity }\end{array}$ & PTSD \\
\hline
\end{tabular}


RESILIENCE IN CHILDREN AFTER NATURAL DISASTERS

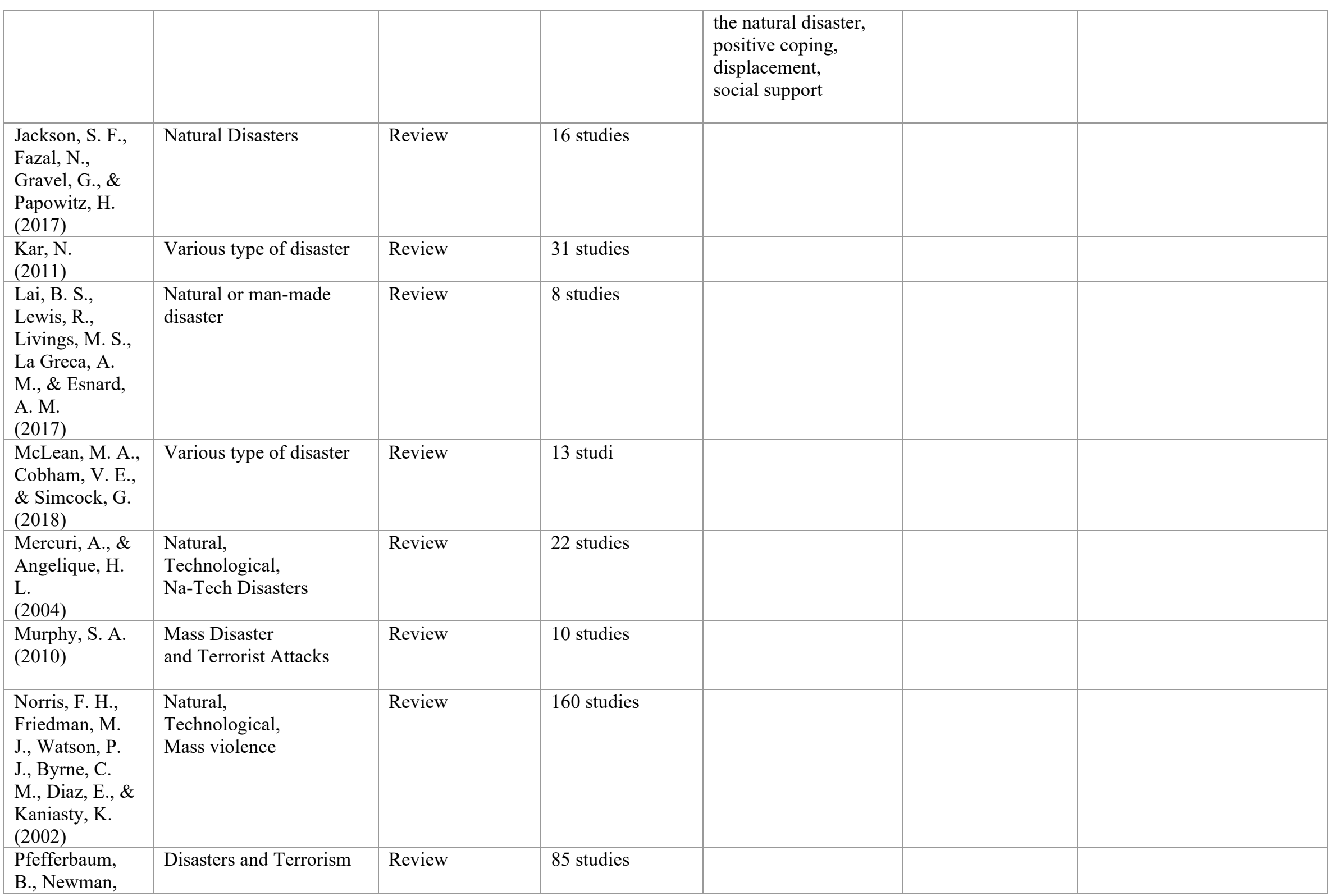




\section{RESILIENCE IN CHILDREN AFTER NATURAL DISASTERS}

\begin{tabular}{|c|c|c|c|c|c|c|}
\hline $\begin{array}{l}\text { E., \& Nelson, S. } \\
\text { D. } \\
(2014)\end{array}$ & & & & & & \\
\hline $\begin{array}{l}\text { Pfefferbaum, } \\
\text { B., Nitiéma, P., } \\
\text { \& Newman, E. } \\
(2019)\end{array}$ & $\begin{array}{l}\text { Accidents, } \\
\text { natural disasters, } \\
\text { terrorism, war }\end{array}$ & Meta-analysis & 24 studies & $\begin{array}{l}\text { Type of disaster } \\
\text { Income } \\
\text { Type of intervention } \\
\text { Lenght of the } \\
\text { intervention }\end{array}$ & & $\begin{array}{l}\text { Intervention Effects on } \\
\text { Depression and/ } \\
\text { or Anxiety }\end{array}$ \\
\hline $\begin{array}{l}\text { Pfefferbaum, } \\
\text { B., Nitiéma, P., } \\
\text { Tucker, P., \& } \\
\text { Newman, E. } \\
(2017) .\end{array}$ & $\begin{array}{l}\text { Natural and Man-Made } \\
\text { Disasters }\end{array}$ & Review & 11 studies & & & \\
\hline $\begin{array}{l}\text { Pfefferbaum, } \\
\text { B., Varma, V., } \\
\text { Nitiéma, P., \& } \\
\text { Newman, E. } \\
\text { (2014) }\end{array}$ & $\begin{array}{l}\text { Disasters and } \\
\text { Terrorism }\end{array}$ & Review & 48 studies & & & \\
\hline $\begin{array}{l}\text { Pfefferbaum, } \\
\text { B., Weems, C. } \\
\text { F., Scott, B. G., } \\
\text { Nitiéma, P., } \\
\text { Noffsinger, M. } \\
\text { A., } \\
\text { Pfefferbaum, R. } \\
\text { L., et al. (2013) }\end{array}$ & $\begin{array}{l}\text { Terrorist attacks, } \\
\text { Tsunami, } \\
\text { Hurricane }\end{array}$ & Review & 165 studies & & & \\
\hline $\begin{array}{l}\text { Pine, D. S., \& } \\
\text { Cohen, J. A. } \\
(2002)\end{array}$ & $\begin{array}{l}\text { Natural and Man-Made } \\
\text { Disasters }\end{array}$ & Review & 32 studies & & & \\
\hline $\begin{array}{l}\text { Rubens, S. L., } \\
\text { Felix, E. D., \& } \\
\text { Hambrick, E. P. } \\
(2018)\end{array}$ & Natural Disasters & Meta-Analysis & 88 studies & Disaster exposure & $\begin{array}{l}\text { Countries with a } \\
\text { medium Human } \\
\text { Development } \\
\text { Index }\end{array}$ & $\begin{array}{l}\text { Internalizing } \\
\text { and Externalizing Problems }\end{array}$ \\
\hline $\begin{array}{l}\text { Saulnier, D. D., } \\
\text { \& Brolin, K. } \\
(2015)\end{array}$ & $\begin{array}{l}\text { Natural and Man-Made } \\
\text { Disasters }\end{array}$ & Review & 47 studies & & & \\
\hline $\begin{array}{l}\text { Tang, B., Liu, } \\
\text { X., Liu, Y., } \\
\text { Xue, C., \& }\end{array}$ & $\begin{array}{l}21 \text { articles on } \\
\text { earthquakes, }\end{array}$ & Meta-analysis & $\begin{array}{l}31 \text { studies on } \\
\text { adults, } 7 \text { on } \\
\text { children }\end{array}$ & $\begin{array}{l}\text { For adults: female; } \\
\text { not married; holding } \\
\text { religious beliefs; poor }\end{array}$ & & Depression \\
\hline
\end{tabular}




\section{RESILIENCE IN CHILDREN AFTER NATURAL DISASTERS}

\begin{tabular}{|c|c|c|c|c|c|}
\hline $\begin{array}{l}\text { Zhang, L. } \\
(2014)\end{array}$ & $\begin{array}{l}7 \text { on } \\
\text { hurricanes/tornadoes/typh } \\
\text { oons, } \\
2 \text { on tsunamis, and } \\
1 \text { on floods. }\end{array}$ & & & $\begin{array}{l}\text { education; prior } \\
\text { trauma; } \\
\text { experiencing fear, } \\
\text { injury, or bereavement } \\
\text { during the disaster; or } \\
\text { losing employment or } \\
\text { property, house } \\
\text { damage } \\
\text { For children: prior } \\
\text { trauma; being trapped; } \\
\text { experiencing injury, } \\
\text { fear, or bereavement } \\
\text { during the disaster; } \\
\text { witnessing } \\
\text { injury/death during the } \\
\text { disaster; } \\
\text { poor social support. }\end{array}$ & \\
\hline $\begin{array}{l}\text { Tolin, D. F., \& } \\
\text { Foa, E. B. } \\
(2006)\end{array}$ & $\begin{array}{l}\text { Natural and Man-Made } \\
\text { Disasters }\end{array}$ & Meta-analyses & 290 studies & Gender & PTSD \\
\hline $\begin{array}{l}\text { Udomratn, P. } \\
\text { (2009) }\end{array}$ & Tsunami & Review & 25 studies & & \\
\hline $\begin{array}{l}\text { Vogel, J. M., \& } \\
\text { Vernberg, E. M. } \\
\text { (1993) }\end{array}$ & $\begin{array}{l}\text { Natural and Man-Made } \\
\text { Disasters }\end{array}$ & Review & 33 studies & & \\
\hline $\begin{array}{l}\text { Wang, C. W., } \\
\text { Chan, C. L. W., } \\
\text { \& Ho, R. T. H. } \\
(2013)\end{array}$ & Natural Disasters & Review & $\begin{array}{l}60 \text { cross sectional } \\
\text { studies; } 25 \\
\text { longitudinal } \\
\text { studies }\end{array}$ & & \\
\hline
\end{tabular}

Note. PTSD = Posttraumatic Stress Disorder; PTS = Posttraumatic Symptoms; CBT = Cognitive-Behavioural Therapy; EMDR = Eye Movement Desensitization and Reprocessing 
Table 3_OSM. Studies on interventions ( $N=29$ studies)

\begin{tabular}{|c|c|c|c|c|c|}
\hline Study & Disaster & Population & Independent variable(s) & $\begin{array}{l}\text { Assessment(s) } \\
\text { (months postdisaster) }\end{array}$ & $\begin{array}{l}\text { Dependent } \\
\text { variable(s) }\end{array}$ \\
\hline $\begin{array}{l}\text { Adúriz, M. E., } \\
\text { Bluthgen, C., \& } \\
\text { Knopfler, C. (2009) }\end{array}$ & $\begin{array}{l}\text { Flood } \\
\text { (Argentina) }\end{array}$ & $\begin{array}{l}124 \text { children } \\
4-17 \text { years }\end{array}$ & $\begin{array}{l}\text { One-session group eye } \\
\text { movement desensitization } \\
\text { and reprocessing (EMDR) }\end{array}$ & $\begin{array}{l}3 \text { months after flood, } \\
3 \text { months follow-up } \\
\text { evaluation session }\end{array}$ & $\begin{array}{l}\text { Distress } \\
\text { Child's Reaction to } \\
\text { Traumatic Events }\end{array}$ \\
\hline $\begin{array}{l}\text { Amin, R., Nadeem, } \\
\text { E., Iqbal, K., } \\
\text { Asadullah, M. A., } \\
\text { \& Hussain (2020) }\end{array}$ & $\begin{array}{l}\text { Flood } \\
\text { (Pakistan) }\end{array}$ & $\begin{array}{l}75 \text { children } \\
7-13 \text { years old }\end{array}$ & $\begin{array}{l}\text { Support for Students } \\
\text { Exposed to Trauma (SSET) } \\
\text { program }\end{array}$ & $\begin{array}{l}2 \text { years after flood, } \\
6 \text { months follow-up } \\
\text { evaluation session }\end{array}$ & $\begin{array}{l}\text { PTSD } \\
\text { resilience } \\
\text { social support }\end{array}$ \\
\hline $\begin{array}{l}\text { Bahar, Z., Ozturk, } \\
\text { M., Beser, A., } \\
\text { Baykara, A., Eker, } \\
\text { G., \& Cakaloz, B. } \\
(2008) .\end{array}$ & $\begin{array}{l}\text { Earthquake } \\
\text { (Turkey) }\end{array}$ & $\begin{array}{l}187 \text { students } \\
7 \text { th grade }\end{array}$ & $\begin{array}{l}\text { Socioeconomic status } \\
\text { Gender } \\
\text { Age } \\
\text { Problem-based group therapy } \\
\text { and occupational therapy }\end{array}$ & $\begin{array}{l}6 \text { months after the } \\
\text { earthquake }\end{array}$ & Depression \\
\hline $\begin{array}{l}\text { Berger R, \& } \\
\text { Gelkopf M. (2009) }\end{array}$ & $\begin{array}{l}\text { Tsunami } \\
\text { (Sri Lanka) }\end{array}$ & $\begin{array}{l}166 \text { students } \\
9-15 \text { years }\end{array}$ & $\begin{array}{l}\text { Intervention (ERASE Stress } \\
\text { Sri Lanka') vs control group } \\
\text { or waiting list } \\
\text { Exposure and previous } \\
\text { traumatic experience } \\
\text { Content of Intervention: } \\
\text { psychoeducational material, } \\
\text { cognitive-behavioral skills, } \\
\text { meditative practices and bio- } \\
\text { energetic exercises utilizing }\end{array}$ & $\begin{array}{l}1 \text { week prior and } 3 \text { months } \\
\text { after the intervention }\end{array}$ & $\begin{array}{l}\text { PTSD, } \\
\text { Functional problems } \\
\text { Somatic complaints } \\
\text { Depression }\end{array}$ \\
\hline
\end{tabular}




\begin{tabular}{|c|c|c|c|c|c|}
\hline & & & $\begin{array}{l}\text { art therapy and narrative } \\
\text { techniques }\end{array}$ & & \\
\hline $\begin{array}{l}\text { Catani C, } \\
\text { Kohiladevy M, Ruf } \\
\text { M, Schauer E, } \\
\text { Elbert T, \& Neuner } \\
\text { F. (2009) }\end{array}$ & $\begin{array}{l}\text { Tsunami } \\
\text { (Sri Lanka) }\end{array}$ & $\begin{array}{l}31 \text { children } \\
8-14 \text { years }\end{array}$ & $\begin{array}{l}6 \text { sessions Narrative } \\
\text { Exposure Therapy for } \\
\text { children (KIDNET) } \\
\text { or } 6 \text { sessions of meditation- } \\
\text { relaxation therapy (MED- } \\
\text { RELAX) }\end{array}$ & $\begin{array}{l}\text { Intervention few weeks } \\
\text { after tsunami } \\
1 \text { month post-test/ } \\
6 \text { months follow-up }\end{array}$ & $\begin{array}{l}\text { PTSD } \\
\text { Level of functioning } \\
\text { Physical health }\end{array}$ \\
\hline $\begin{array}{l}\text { Chemtob, C. M., } \\
\text { Nakashima, J., \& } \\
\text { Carlson, J. G. } \\
\text { (2001) }\end{array}$ & $\begin{array}{l}\text { Hurricane } \\
\text { (Hawaii) }\end{array}$ & $\begin{array}{l}32 \text { children } \\
6-12 \text { years }\end{array}$ & $\begin{array}{l}3 \text { sessions of EMDR } \\
\text { Experienced hurricane- } \\
\text { related symptoms } \\
\text { PTSD }\end{array}$ & $\begin{array}{l}3,5 \text { years after hurricane } \\
\text { Pretreatment, } \\
\text { Posttreatment, and Follow- } \\
\text { Up } 6 \text { months }\end{array}$ & $\begin{array}{l}\text { PTSD } \\
\text { Anxiety } \\
\text { Depression }\end{array}$ \\
\hline $\begin{array}{l}\text { Fernandez, I. } \\
\text { (2007) }\end{array}$ & $\begin{array}{l}\text { Earthquake } \\
\text { (Italy) }\end{array}$ & $\begin{array}{l}22 \text { children, } \\
6 \text { years }\end{array}$ & $\begin{array}{l}3 \text { sessions of EMDR } \\
\text { PTSD }\end{array}$ & $\begin{array}{l}1 \text { month, } 3 \text { months and a } \\
\text { year after } \\
\text { the event }\end{array}$ & PTSD \\
\hline $\begin{array}{l}\text { Field, T. M., } \\
\text { Seligman, S., } \\
\text { Scafidi, F., \& } \\
\text { Schanberg, S. } \\
\text { (1996) }\end{array}$ & $\begin{array}{l}\text { Hurricane } \\
\text { Florida }\end{array}$ & $\begin{array}{l}60 \text { children } \\
1 \text { st }-5 \text { th graders }\end{array}$ & $\begin{array}{l}\text { Massage therapy or video } \\
\text { attention group (control } \\
\text { group) } \\
\text { Hurricane impact } \\
\text { PTSD } \\
\text { Anxiety } \\
\text { Salivary } \\
\text { cortisol levels }\end{array}$ & & $\begin{array}{l}\text { Anxiety } \\
\text { Depression } \\
\text { Mood state } \\
\text { Salivary cortisol levels }\end{array}$ \\
\hline $\begin{array}{l}\text { Fu C, Leoutsakos } \\
\text { JM, \& Underwood } \\
\text { C. (2013) }\end{array}$ & $\begin{array}{l}\text { Earthquake } \\
\text { (Sichuan) }\end{array}$ & $\begin{array}{l}4120 \text { children } \\
6-16 \text { years }\end{array}$ & Sociodemographics factors & 1 year after disaster & PTSD \\
\hline
\end{tabular}




\begin{tabular}{|c|c|c|c|c|c|}
\hline & & & $\begin{array}{l}\text { Earthquake-related risk } \\
\text { exposure } \\
\text { Resilience }\end{array}$ & & \\
\hline $\begin{array}{l}\text { Garfin, D. R., } \\
\text { Silver, R. C., Gil- } \\
\text { Rivas, V., Guzmán, } \\
\text { J., Murphy, J. M., } \\
\text { Cova, F., ... } \\
\text { Guzmán, M. P. } \\
\text { (2014) }\end{array}$ & $\begin{array}{l}\text { Earthquake } \\
\text { (Chile) }\end{array}$ & $\begin{array}{l}117 \text { children } \\
7-8 \text { years, }\end{array}$ & $\begin{array}{l}\text { Exposure } \\
\text { Family Context School- } \\
\text { Based Mental Health } \\
\text { Program } \\
\text { Gender }\end{array}$ & 9 months post-earthquake & $\begin{array}{l}\text { PTS } \\
\text { Earthquake-related } \\
\text { worry }\end{array}$ \\
\hline $\begin{array}{l}\text { Giannopoulou, I., } \\
\text { Dikaiakou, A., \& } \\
\text { Yule, W. (2006) }\end{array}$ & $\begin{array}{l}\text { Earthquake } \\
\text { (Athens) }\end{array}$ & $\begin{array}{l}20 \text { children } \\
8-12 \text { years }\end{array}$ & $\begin{array}{l}6 \text { weeks Group cognitive- } \\
\text { behavioural intervention } \\
\text { Group } 1(\mathrm{~N}=10) \text {, which } \\
\text { started treatment } 2 \text { months } \\
\text { after the earthquake and } \\
\text { Group } 2(\mathrm{~N}=10) \text {, which } \\
\text { started treatment after } 4 \\
\text { months } \\
\text { PTSD }\end{array}$ & $\begin{array}{l}\text { Intervention: } \\
2 \text { months and } 4 \text { months } \\
\text { after earthquake } \\
\text { Assessment: } \\
\text { before treatment (T1), } \\
\text { immediately after } \\
\text { completing the treatment } \\
\text { (T2), at first } \\
\text { follow-up } \\
\text { 18 MONTHS } \\
\text { (T3) and at the second } \\
\text { follow-up } \\
\text { 4-year follow-up. }\end{array}$ & $\begin{array}{l}\text { PTSD } \\
\text { Depression } \\
\text { Psychosocial } \\
\text { functioning. }\end{array}$ \\
\hline $\begin{array}{l}\text { Goenjian, A. } \\
\text { (1993) }\end{array}$ & $\begin{array}{l}\text { Earthquake } \\
\text { (Armenia) }\end{array}$ & $\begin{array}{l}582 \text { children } \\
6-12 \text { years }\end{array}$ & $\begin{array}{l}\text { Mental Health Relief } \\
\text { Programme }\end{array}$ & 3-6 months after earthquake & $\begin{array}{l}\text { PTSD } \\
\text { Major depressive } \\
\text { disorder }\end{array}$ \\
\hline $\begin{array}{l}\text { Graham, R. A., } \\
\text { Osofsky, J. D., } \\
\text { Osofsky, H. J., \& } \\
\text { Hansel, T. C. } \\
\text { (2017) }\end{array}$ & $\begin{array}{l}\text { Hurricane } \\
\text { (Louisiana) }\end{array}$ & $\begin{array}{l}112 \text { students } \\
8-17 \text { years }\end{array}$ & $\begin{array}{l}\text { Cognitive Behavioral } \\
\text { Intervention for Trauma in } \\
\text { Schools (CBITS) } \\
\text { Individual treatment at } \\
\text { school including: relaxation, }\end{array}$ & 4 months after Hurricane & $\begin{array}{l}\text { Anxiety } \\
\text { Depression } \\
\text { Anger } \\
\text { PTS } \\
\text { Dissociation }\end{array}$ \\
\hline
\end{tabular}




\begin{tabular}{|c|c|c|c|c|c|}
\hline & & & $\begin{array}{l}\text { coping, and problem solving } \\
\text { skill development; trauma } \\
\text { narratives; and cognitive } \\
\text { restructuring }\end{array}$ & & \\
\hline $\begin{array}{l}\text { Hansel, T. C., } \\
\text { Osofsky, H. J., } \\
\text { Osofsky, J. D., \& } \\
\text { Speier, A. H. } \\
(2019)\end{array}$ & $\begin{array}{l}\text { Hurricane } \\
\text { (USA) }\end{array}$ & $\begin{array}{l}4.593 \text { children } 7-14 \\
\text { years old }\end{array}$ & $\begin{array}{l}\text { School-based trauma } \\
\text { interventions }\end{array}$ & & PTSD \\
\hline $\begin{array}{l}\text { Ho, R. T. H., Lai, } \\
\text { A. H. Y., Lo, P. H. } \\
\text { Y., Nan, J. K. M., } \\
\text { \& Pon, A. K. L. } \\
(2016)\end{array}$ & $\begin{array}{l}\text { Earthquake } \\
\text { (Sichuan) }\end{array}$ & $\begin{array}{l}112 \text { children } \\
\text { Grade } 4\end{array}$ & $\begin{array}{l}\text { Gender } \\
\text { Self-Efficacy, Peer } \\
\text { Support, and Anxiety } \\
\text { Arts and } \\
\text { play support program } \\
\text { (promotion of self-efficacy, } \\
\text { peer support positive } \\
\text { thinking and problem } \\
\text { solving) } \\
\text { Control group }\end{array}$ & $\begin{array}{l}\text { Baseline: } 1 \text { year after the } \\
\text { earthquake } \\
\text { Post test: } 2 \text { years after }\end{array}$ & $\begin{array}{l}\text { Self-Efficacy } \\
\text { Peer Support } \\
\text { Anxiety }\end{array}$ \\
\hline $\begin{array}{l}\text { Jarero, I., Artigas, } \\
\text { L., \& Hartung, J. } \\
\text { (2006). }\end{array}$ & $\begin{array}{l}\text { Hurricane } \\
\text { (Mexico) }\end{array}$ & $\begin{array}{l}44 \text { children } \\
8-15 \text { years }\end{array}$ & $\begin{array}{l}\text { EMDR Integrative Group } \\
\text { Treatment } \\
\text { Protocol }\end{array}$ & 1 months after hurricane & Distress \\
\hline $\begin{array}{l}\text { Karairmak, Ö., \& } \\
\text { Aydin, G. (2008). }\end{array}$ & $\begin{array}{l}\text { Earthquake } \\
\text { (Turkey) }\end{array}$ & $\begin{array}{l}166 \text { elementary } \\
\text { school students } \\
4^{\text {th }}-8^{\text {th }} \text { grade }\end{array}$ & $\begin{array}{l}\text { Gender } \\
\text { Group (victims vs non } \\
\text { victims) } \\
\text { Activity-based cognitive fear } \\
\text { reduction (ABCF): } \\
\text { understand that natural } \\
\text { disasters would occur and } \\
\text { develop coping skills and } \\
\text { strategies for normalization }\end{array}$ & & $\begin{array}{l}\text { Earthquake-related } \\
\text { fears }\end{array}$ \\
\hline $\begin{array}{l}\text { Klontz, B. T., } \\
\text { Bivens, A., } \\
\text { Michels, S., } \\
\text { DeLeon, P. H., \& } \\
\text { Tom, L. (2015). }\end{array}$ & $\begin{array}{l}\text { Hurricane } \\
\text { (Hawaii) }\end{array}$ & $\begin{array}{l}179 \text { children } \\
5-16 \text { years }\end{array}$ & $\begin{array}{l}\text { School-based behavioral } \\
\text { health services: Mokihana } \\
\text { Program }\end{array}$ & $\begin{array}{l}1 \text { year follow up after } \\
\text { treatment }\end{array}$ & $\begin{array}{l}\text { Behavioral problems } \\
\text { and adaptive } \\
\text { functioning }\end{array}$ \\
\hline
\end{tabular}




\begin{tabular}{|c|c|c|c|c|c|}
\hline & & & $\begin{array}{l}\text { Focus on responding to the } \\
\text { needs of the child and family } \\
\text { Behavioral problems and } \\
\text { adaptive functioning }\end{array}$ & & \\
\hline $\begin{array}{l}\text { Liu, Z., Zhu, Z., } \\
\text { Kao, H. S. R., } \\
\text { Zong, Y., Tang, S., } \\
\text { Xu, M., .. Wang, } \\
\text { R. (2014) }\end{array}$ & $\begin{array}{l}\text { Earthquake } \\
\text { (China) }\end{array}$ & $\begin{array}{l}\text { Experiment } 1 \text { : } \\
210 \text { children } \\
\text { Experiment } 2 \\
80 \text { children } \\
11 \text { years }\end{array}$ & $\begin{array}{l}\text { Group Calligraphy } \\
\text { Intervention } \\
\text { Group Control } \\
\text { Gender } \\
\text { Age } \\
\text { Time }\end{array}$ & $\begin{array}{l}1 \text { year after exposure to } \\
\text { natural disaster } \\
\text { Post intervention } \\
\text { assessment: } \\
\text { after } 15 \text { days } \\
\text { after } 30 \text { days }\end{array}$ & $\begin{array}{l}\text { Intrusion Avoidance } \\
\text { Hyperarousal } \\
\text { symptoms } \\
\text { PTSD } \\
\text { Salivary cortisol }\end{array}$ \\
\hline $\begin{array}{l}\text { Pityaratstian, N., } \\
\text { Piyasil, V., } \\
\text { Ketumarn, P., } \\
\text { Sitdhiraksa, N., } \\
\text { Ularntinon, S., \& } \\
\text { Pariwatcharakul, P. } \\
\text { (2015). }\end{array}$ & Tsunami & $\begin{array}{l}36 \text { children } \\
10-15 \text { years who had } \\
\text { been diagnosed with } \\
\text { PSTD } 4 \text { years after } \\
\text { the tsunami were } \\
\text { randomly allocated } \\
\text { to either }\end{array}$ & $\begin{array}{l}\text { Diagnosis with PSTD } 4 \text { years } \\
\text { after tsunami } \\
\text { CBT (cognitive behavioural } \\
\text { therapy) or wait list }\end{array}$ & $\begin{array}{l}\text { Posttreatment and 1-month } \\
\text { follow up }\end{array}$ & PTSD \\
\hline $\begin{array}{l}\text { Powell, T. M., \& } \\
\text { Bui, T. (2016). }\end{array}$ & $\begin{array}{l}\text { Tornado } \\
\text { (Oklahoma) }\end{array}$ & $\begin{array}{l}110 \text { youth } \\
11-15 \text { years }\end{array}$ & $\begin{array}{l}\text { Journey of Hope Intervention } \\
\text { group } \\
\text { Control group } \\
\text { Coping skills } \\
\text { self-efficacy, } \\
\text { prosocial behaviors, distress. }\end{array}$ & $\begin{array}{l}9 \text { months after the } \\
\text { tornado } \\
1 \text { month after post-test }\end{array}$ & $\begin{array}{l}\text { Coping skills } \\
\text { Self-efficacy } \\
\text { Prosocial behaviors, } \\
\text { Distress }\end{array}$ \\
\hline $\begin{array}{l}\text { Powell, T., \& } \\
\text { Thompson, S. J. } \\
\text { (2016) }\end{array}$ & $\begin{array}{l}\text { Tornado } \\
\text { (Alabama) }\end{array}$ & $\begin{array}{l}102 \text { students } \\
8-12 \text { years }\end{array}$ & $\begin{array}{l}\text { Journey of Hope }(\mathrm{JoH}) \text {, a } \\
\text { school-based intervention } \\
\text { Control group } \\
\text { Coping skills peer } \\
\text { relationships }\end{array}$ & $\begin{array}{l}5-8 \text { months after the } \\
\text { disaster }\end{array}$ & $\begin{array}{l}\text { Coping skills } \\
\text { Peer relationships }\end{array}$ \\
\hline $\begin{array}{l}\text { Rousseau, C., } \\
\text { Benoit, M., } \\
\text { Lacroix, L., \& } \\
\text { Gauthier, M. F. } \\
\text { (2009). }\end{array}$ & $\begin{array}{l}\text { Tsunami } \\
\text { (South Asia) }\end{array}$ & $\begin{array}{l}105 \text { children } \\
4-6 \text { years }\end{array}$ & $\begin{array}{l}\text { Sandplay program } \\
\text { Group } \\
\text { Control Group } \\
\text { Socio-economics factors }\end{array}$ & $\begin{array}{l}\text { Pretest } \\
\text { just before the tsunami } \\
\text { Posttest } 6 \text { months after } \\
\text { disaster }\end{array}$ & $\begin{array}{l}\text { Emotional and } \\
\text { behavioral symptoms } \\
\text { Anxiety Depression } \\
\text { Distress } \\
\text { Social Impairment }\end{array}$ \\
\hline
\end{tabular}




\begin{tabular}{|c|c|c|c|c|c|}
\hline & & & & & Burden for others \\
\hline $\begin{array}{l}\text { Salloum, A., \& } \\
\text { Overstreet, S. } \\
(2008)\end{array}$ & $\begin{array}{l}\text { Hurricane } \\
\text { (New Orleans) }\end{array}$ & $\begin{array}{l}56 \text { children } \\
7-12 \text { years }\end{array}$ & $\begin{array}{l}\text { Group community-based } \\
\text { grief and trauma intervention } \\
\text { Group individual } \\
\text { intervention } \\
\text { disaster-related exposure } \\
\text { Demographic information } \\
\text { Time of assessment } \\
\text { PTSD } \\
\text { Disaster-related } \\
\text { Exposure } \\
\text { Depression } \\
\text { Traumatic grief } \\
\text { Distress }\end{array}$ & $\begin{array}{l}4 \text { months post-Hurricane } \\
\text { preintervention, } \\
\text { postintervention, } \\
3 \text { weeks follow-up }\end{array}$ & $\begin{array}{l}\text { PTSD } \\
\text { Depression } \\
\text { Traumatic grief } \\
\text { Distress }\end{array}$ \\
\hline Shen, Y. J. (2002) & $\begin{array}{l}\text { Earthquake } \\
\text { (Taiwan) }\end{array}$ & $\begin{array}{l}65 \text { children } \\
8-12 \text { years }\end{array}$ & $\begin{array}{l}\text { Child-centered group play } \\
\text { therapy } \\
\text { Control group }\end{array}$ & $\begin{array}{l}\text { Pretests and posttests } \\
\text { within } 2 \text { weeks of the } \\
\text { treatment. }\end{array}$ & $\begin{array}{l}\text { Anxiety } \\
\text { Depression } \\
\text { Adjustment }\end{array}$ \\
\hline $\begin{array}{l}\text { Stasiak, K., Merry, } \\
\text { S. N., Frampton, } \\
\text { C., \& Moor, S. } \\
(2016)\end{array}$ & $\begin{array}{l}\text { Earthquake } \\
\text { (New Zealand) }\end{array}$ & $\begin{array}{l}42 \text { children } 7-18 \\
\text { years old }\end{array}$ & $\begin{array}{l}\text { Online cognitive behaviour } \\
\text { therapy (CBT) }\end{array}$ & $\begin{array}{l}14-20 \text { months after the } \\
\text { earthquake }\end{array}$ & $\begin{array}{l}\text { Anxiety } \\
\text { Depression } \\
\text { Health-related quality } \\
\text { of life }\end{array}$ \\
\hline $\begin{array}{l}\text { Trentini, C., } \\
\text { Lauriola, M., } \\
\text { Giuliani, A., } \\
\text { Maslovaric, G., } \\
\text { Tambelli, R., } \\
\text { Fernandez, I., \& } \\
\text { Pagani, M. (2018) }\end{array}$ & $\begin{array}{l}\text { Earthquake } \\
\text { (Italy) }\end{array}$ & $\begin{array}{l}332 \text { children } 5-13 \\
\text { years old }\end{array}$ & $\begin{array}{l}\text { EMDR Integrative Group } \\
\text { Treatment Protocol } \\
\text { (EMDR-IGTP) } \\
\text { Time } \\
\text { Elapsed } \\
\text { Age } \\
\text { Gender }\end{array}$ & 3 weeks after earthquake & $\begin{array}{l}\text { Distress, } \\
\text { Anxiety, } \\
\text { Depression, } \\
\text { Anger } \\
\text { Need Help }\end{array}$ \\
\hline $\begin{array}{l}\text { Vijayakumar, L., } \\
\text { Kannan, G. K., } \\
\text { Ganesh Kumar, B., } \\
\text { \& Devarajan, P. } \\
\text { (2006). }\end{array}$ & $\begin{array}{l}\text { Tsunami } \\
\text { (Asia) }\end{array}$ & $\begin{array}{l}135 \text { children } \\
11-14 \text { years }\end{array}$ & $\begin{array}{l}\text { Socio-demographic factors } \\
\text { Exposure } \\
\text { Psychopathology of the } \\
\text { children } \\
\text { Family mental health } \\
\text { Psychosocial intervention }\end{array}$ & $\begin{array}{l}1 \text { year after tsunami } \\
\text { before and after } \\
\text { intervention ( } 9 \text { months after } \\
\text { the initial assessment) }\end{array}$ & $\begin{array}{l}\text { PTSD } \\
\text { Hyperactivity }\end{array}$ \\
\hline
\end{tabular}




\begin{tabular}{|c|c|c|c|c|c|}
\hline $\begin{array}{l}\text { Wolmer, L., Laor, } \\
\text { N., Dedeoglu, C., } \\
\text { Siev, J., \& Yazgan, } \\
\text { Y. (2005). }\end{array}$ & $\begin{array}{l}\text { Earthquake } \\
\text { (Turkey) }\end{array}$ & $\begin{array}{l}287 \text { children } \\
9-17 \text { years }\end{array}$ & $\begin{array}{l}\text { School Reactivation Program } \\
\text { Socio-demographic } \\
\text { information } \\
\text { Stressful/traumatic episodes } \\
\text { before and after the } \\
\text { earthquake } \\
\text { Exposure } \\
\text { Academic performance, } \\
\text { Social behavior } \\
\text { PTSD } \\
\text { Time }\end{array}$ & $\begin{array}{l}3.5 \text { years after the } \\
\text { earthquake } \\
\text { post-intervention } \\
\text { assessment } 3 \text { years later }\end{array}$ & $\begin{array}{l}\text { PTSD } \\
\text { Grief } \\
\text { Dissociation }\end{array}$ \\
\hline
\end{tabular}

Note. PTSD = Posttraumatic Stress Disorder; PTS = Posttraumatic Symptoms; CBT = Cognitive-Behavioural Therapy; EMDR = Eye Movement Desensitization and Reprocessing 
RESILIENCE IN CHILDREN AFTER NATURAL DISASTERS

Table 4_OSM. Frequency of natural disasters $(N=294$ studies $)$

\begin{tabular}{|l|r|}
\hline \multicolumn{2}{|c|}{ DISASTER } \\
\hline Earthquake & 1126 \\
\hline Hurricane & 92 \\
\hline Tsunami & 27 \\
\hline Flood & 20 \\
\hline Tornado & 15 \\
\hline Cyclone/ Typhoon & 8 \\
\hline Ice Storm & 4 \\
\hline War/Violence & 3 \\
\hline Lightning Strike & 3 \\
\hline Gulf Oil Spill & 2 \\
\hline Storm & 5 \\
\hline Eruption & 2 \\
\hline
\end{tabular}

Note. The sum is higher than the total number of studies, because some studies included the analysis of two or more types of disasters. 
RESILIENCE IN CHILDREN AFTER NATURAL DISASTERS

Table 5_OSM. Study design $(N=294$ studies $)$

\begin{tabular}{|l|r|}
\hline \multicolumn{2}{|c|}{ DESIGN } \\
\hline Cross-sectional & 182 \\
\hline Longitudinal & 78 \\
\hline Pre-post without control group & 13 \\
\hline Experimental with control group & 11 \\
\hline Pre-post and longitudinal & 6 \\
\hline Experimental without control group & 2 \\
\hline Pre-post with control group & 1 \\
\hline $\begin{array}{l}\text { Experimental (with control group) } \\
\text { longitudinal }\end{array}$ & 1 \\
\hline
\end{tabular}


Table 6_OSM. Level of analysis $(N=294$ studies $)$

\begin{tabular}{|l|r|}
\hline \multicolumn{2}{|c|}{ LEVEL } \\
\hline Individual & 172 \\
\hline Individual and Interpersonal & 108 \\
\hline Individual and Intergroup & 5 \\
\hline Individual, Interpersonal and Intergroup & 6 \\
\hline Individual, Interpersonal and Group & 1 \\
\hline $\begin{array}{l}\text { Individual, Interpersonal, Group and } \\
\text { Intergroup }\end{array}$ & 1 \\
\hline
\end{tabular}

Note. Studies included in one category are not included in other categories. 
RESILIENCE IN CHILDREN AFTER NATURAL DISASTERS

Table 7_OSM. Frequency of main independent variables ( $N=294$ studies) 


\section{RESILIENCE IN CHILDREN AFTER NATURAL DISASTERS}

\begin{tabular}{|c|c|}
\hline \multicolumn{2}{|c|}{ INDEPENDENT VARIABLES } \\
\hline Exposure & 168 \\
\hline Gender & 139 \\
\hline Age & 132 \\
\hline Stress/Distress & 36 \\
\hline Support & 34 \\
\hline Time & 31 \\
\hline Social Level (Status, Living Conditions, Demographic, Socioeconomic factors) & 35 \\
\hline Type Of Disaster & 24 \\
\hline Coping/Appraisal/Resilience & 23 \\
\hline Parental Stress & 25 \\
\hline Family Functioning & 20 \\
\hline Ethnicity & 21 \\
\hline PTSD & 20 \\
\hline Impact of Event & 17 \\
\hline Anxiety & 15 \\
\hline School Grade & 16 \\
\hline Material Loss & 14 \\
\hline Parental Loss & 12 \\
\hline Depression & 13 \\
\hline Prenatal Exposure & 9 \\
\hline Parents Level of Education & 11 \\
\hline Previous Traumatic Experiences & 7 \\
\hline Disaster Relocation & 6 \\
\hline Children Pre disaster disorders & 6 \\
\hline Life Threat & 4 \\
\hline Child Level of education & 5 \\
\hline Rumination & 4 \\
\hline Social Connection & 3 \\
\hline Parents Pre disaster Disorders & 4 \\
\hline Religion & 4 \\
\hline Injury and Illness & 3 \\
\hline Academic Skills & 2 \\
\hline Abuse/Neglect & 1 \\
\hline Sleeping Problems & 2 \\
\hline Verbal Ability & 1 \\
\hline Children's Trauma Recollections & 1 \\
\hline Marital Status & 6 \\
\hline Maternal Prenatal Depression & 5 \\
\hline Maternal Anxiety & 3 \\
\hline Parental Conflict & 1 \\
\hline Maternal Cortisol Concentration & 1 \\
\hline Maternal Prenatal Stress & 4 \\
\hline
\end{tabular}


RESILIENCE IN CHILDREN AFTER NATURAL DISASTERS

Substance Use

Parent-Child Conflict

Maternal Age

Birth Complications

Stressful Life Events

Note. $\mathrm{PTSD}=$ posttraumatic stress disorder. The sum is higher than the total number of studies, because some studies included two or more independent variables. 
Table 8_OSM. Frequency of main dependent variables ( $\mathrm{N}=294$ studies)

\begin{tabular}{|l|r|}
\hline \multicolumn{2}{|c|}{ DEPENDENT VARIABLES } \\
\hline PTSD & 119 \\
\hline Depression/ Thoughts of Suicide & 43 \\
\hline Anxiety (GAD) & 30 \\
\hline Adaptation Score, Adjustment Disorder & 22 \\
\hline PTSS & 21 \\
\hline Internalizing/Externalizing Problems & 15 \\
\hline Post Traumatic Growth & 14 \\
\hline Behavioural and Emotional Problems & 12 \\
\hline Physical Health (Somatic Symptoms, Ecc.) & 12 \\
\hline Coping/Appraisal & 11 \\
\hline Psychological Impact and Reactions & 9 \\
\hline Distress & 9 \\
\hline Mental Disorders & 9 \\
\hline Parental Health & 6 \\
\hline Cognitive Functions & 7 \\
\hline Temperamental and Personal Characteristics & 8 \\
\hline School Performance & 4 \\
\hline Rumination and Intrusive Thoughts & 3 \\
\hline Family Functioning & 3 \\
\hline Memory Functions & 2 \\
\hline Social Problems Risk & 2 \\
\hline Tom and Metacognition & 2 \\
\hline Psychiatric Disorders & 2 \\
\hline Contact and Helping Intentions & 2 \\
\hline Child Cortisol Concentration & 1 \\
\hline
\end{tabular}




\begin{tabular}{|l|r|}
\hline Child Motor Development & 1 \\
\hline Resilience & 1 \\
\hline Neural Reactivity & 1 \\
\hline Electrodermal Activity & 1 \\
\hline
\end{tabular}

Note. $\mathrm{PTSD}=$ Posttraumatic Stress Disorder; PTSS= Posttraumatic Stress Symptoms; GAD= Generalized Anxiety Disorder. The sum is higher than the total number of studies, because some studies included two or more dependent variables. 
Table 9_OSM. Frequency of the geographical distribution of the studies ( $N=294$ studies).

\begin{tabular}{|l|l|}
\hline \multicolumn{2}{|l|}{ GEOGRAPHICAL DISTRIBUTION OF THE STUDIES } \\
\hline USA & 122 \\
\hline China & 50 \\
\hline Turkey & 18 \\
\hline Japan & 17 \\
\hline Southeast Asia & 15 \\
\hline Australia & 10 \\
\hline Italy & 8 \\
\hline Haiti & 7 \\
\hline Armenia & 6 \\
\hline India & 5 \\
\hline Sri Lanka & 5 \\
\hline Puerto Rico & 5 \\
\hline Greece & 5 \\
\hline Canada & 4 \\
\hline Chile & 3 \\
\hline Nepal & 2 \\
\hline Pakistan & \\
\hline Iceland & 3 \\
\hline
\end{tabular}


RESILIENCE IN CHILDREN AFTER NATURAL DISASTERS

\begin{tabular}{|l|l|}
\hline Taiwan & 2 \\
\hline Canada & 4 \\
\hline Chile & 3 \\
\hline Nepal & 3 \\
\hline Pakistan & 2 \\
\hline Iceland & 2 \\
\hline Taiwan & 2 \\
\hline Poland & 1 \\
\hline Bangladesh & 1 \\
\hline New Zealand & 1 \\
\hline Iran & 1 \\
\hline Antilles & 1 \\
\hline Tibet & 1 \\
\hline
\end{tabular}




\section{References of the studies included in the review}

Adebäck, P., Schulman, A., \& Nilsson, D. (2017). Children exposed to a natural disaster: Psychological consequences eight years after 2004 tsunami. Nordic Journal of Psychiatry, 72, 7581. doi:10.1080/08039488.2017.1382569

Adúriz, M. E., Bluthgen, C., \& Knopfler, C. (2009). Helping child flood victims using group EMDR intervention in Argentina: Treatment outcome and gender differences. International Journal of Stress Management, 16, 138-153. doi:10.1037/a0014719

Agustini, E. N., Asniar, I., \& Matsuo, H. (2011). The prevalence of long-term post-traumatic stress symptoms among adolescents after the tsunami in Aceh. Journal of Psychiatric And Mental Health Nursing, 18, 543-549. doi: 10.1111/j.1365-2850.2011.01702.x

Amin, R., Nadeem, E., Iqbal, K., Asadullah, M. A., \& Hussain, B. (2020). Support for Students Exposed to Trauma (SSET) Program: An Approach for Building Resilience and Social Support Among Flood-Impacted Children. School Mental Health, 12, 493-506. doi:10.1007/s12310-020$09373-y$

Andrades, M., García, F. E., Calonge, I., \& Martínez-Arias, R. (2018). Posttraumatic growth in children and adolescents exposed to the 2010 earthquake in Chile and its relationship with rumination and posttraumatic stress symptoms. Journal of Happiness Studies, 19, 1505-1517. doi:10.1007/s10902-017-9885-7

Andrades, M., García, F. E., Reyes-Reyes, A., Martínez-Arias, R., \& Calonge, I. (2016). Psychometric properties of the Posttraumatic Growth Inventory for Children in Chilean population 
RESILIENCE IN CHILDREN AFTER NATURAL DISASTERS

affected by the earthquake of 2010. American Journal of Orthopsychiatry, 86, 686-692. doi:10.1037/ort0000182

Anthony, J. L., Lonigan, C. J., \& Hecht, S. A. (1999). Dimensionality of posttraumatic stress disorder symptoms in children exposed to disaster: results from confirmatory factor analyses. Journal of abnormal psychology, 108, 326-336. doi:10.1037//0021-843X.111.4.637

Arnberg, F. K., Gudmundsdóttir, R., Butwicka, A., Fang, F., Lichtenstein, P., Hultman, C. M., \& Valdimarsdóttir, U. A. (2015). Psychiatric disorders and suicide attempts in Swedish survivors of the 2004 southeast Asia tsunami: a 5 year matched cohort study. The Lancet Psychiatry, 2, 817824. doi:10.1016/S2215-0366(15)00124-8

Asarnow, J., Glynn, S., Pynoos, R. S., Nahum, J., Guthrie, D., Cantwell, D. P., \& Franklin, B. (1999). When the earth stops shaking: Earthquake sequelae among children diagnosed for pre-earthquake psychopathology. Journal of the American Academy of Child \& Adolescent Psychiatry, 38, 10161023.doi:10.1097/00004583-199908000-00018

Austin, M. P., Christl, B., McMahon, C., Kildea, S., Reilly, N., Yin, C., ... \& King, S. (2017). Moderating effects of maternal emotional availability on language and cognitive development in toddlers of mothers exposed to a natural disaster in pregnancy: The QF2011 Queensland Flood Study. Infant Behavior and Development, 49, 296-309.doi:10.1016/j.infbeh.2017.10.005

Ayub, M., Poongan, I., Masood, K., Gul, H., Ali, M., Farrukh, A., ... \& Naeem, F. (2012). Psychological morbidity in children 18 months after Kashmir Earthquake of 2005. Child Psychiatry \& Human Development, 43, 323-336.doi:10.1007/s10578-011-0267-9 
Azarian, A., Miller, T. W., \& Skriptchenko-Gregorian, V. (1996). Baseline assessment of children traumatized by the Armenian earthquake. Child Psychiatry and Human Development, 27, 29-41. doi:10.1007/BF02353444

Azarian, A., Skriptchenko-Gregorian, V., Miller, T. W., \& Kraus, R. F. (1994). Childhood trauma in victims of the Armenian earthquake. Journal of Contemporary Psychotherapy, 24, 77-85. doi:10.1007/BF02310246

Bahar, Z., ÖZtürk, M., Beşer, A., Baykara, A., Eker, G., \& Çakaloz, B. (2008). Evaluation of interventions based on depression sign scores of adolescents. Social Behavior and Personality: An International Journal, 36, 123-134. doi:10.2224/sbp.2008.36.1.123

Bahrick, L. E., Parker, J. F., Fivush, R., \& Levitt, M. (1998). The effects of stress on young children's memory for a natural disaster. Journal of Experimental Psychology: Applied, 4, 308-331. doi:10.1016/S1041-6080(97)90004-2

Bal, A. (2008). Post-traumatic stress disorder in Turkish child and adolescent survivors three years after the Marmara earthquake. Child and Adolescent Mental Health, 13, 134139.doi:10.1111/j.1475-3588.2007.00469.x

Bal, A., \& Jensen, B. (2007). Post-traumatic stress disorder symptom clusters in Turkish child and adolescent trauma survivors. European Child \& Adolescent psychiatry, 16, 449-457. doi:10.1007/s00787-007-0618-z

Bauer, P. J., Burch, M. M., Van Abbema, D. L., \& Ackil, J. K. (2007). Talking about twisters: Relations between mothers' and children's contributions to conversations about a devastating tornado. Journal of Cognition and Development, 8, 371-399. doi:10.1080/15248370701612936 
Bauer, P. J., Stark, E. N., Lukowski, A. F., Rademacher, J., Van Abbema, D. L., \& Ackil, J. K. (2005). Working together to make sense of the past: Mothers' and children's use of internal states language in conversations about traumatic and nontraumatic events. Journal of Cognition and Development, 6, 463-488. doi:10.1207/s15327647jcd0604_2

Belter, R. W., Dunn, S. E., \& Jeney, P. (1991). The psychological impact of Hurricane Hugo on children: A needs assessment. Advances in Behaviour Research and Therapy, 13, 155-161. doi:10.1016/0146-6402(91)90003-S

Berger, R., \& Gelkopf, M. (2009). School-based intervention for the treatment of tsunami-related distress in children: A quasi-randomized controlled trial. Psychotherapy and Psychosomatics, 78, 364-371. doi:10.1159/000235976

Bhushan, B., \& Sathya Kumar, J. (2007). Emotional distress and posttraumatic stress in children surviving the 2004 tsunami. Journal of Loss and Trauma,12, 245-257. doi:10.1080/15325020600945996

Blanc, J., Bui, E., Mouchenik, Y., Derivois, D., \& Birmes, P. (2015). Prevalence of post-traumatic stress disorder and depression in two groups of children one year after the January 2010 earthquake in Haiti. Journal of affective disorders, 172, 121-126. doi:10.1016/j.jad.2014.09.055

Bödvarsdóttir, Í., Elklit, A., \& Gudmundsdóttir, D. B. (2006). Post-traumatic stress reactions in children after two large earthquakes in Iceland. Nordic Psychology, 58, 91-107. doi:10.1027/19012276.58.2.91

Bokszczanin, A. (2008). Parental support, family conflict, and overprotectiveness: Predicting PTSD symptom levels of adolescents 28 months after a natural disaster. Anxiety, Stress, \& Coping, 21, $325-335$. 
RESILIENCE IN CHILDREN AFTER NATURAL DISASTERS

Bradburn, I. S. (1991). After the earth shook: Children's stress symptoms 6-8 months after a disaster. Advances in Behaviour Research and Therapy, 13, 173-179. doi:10.1016/01466402(91)90005-U

Bronfenbrenner, U. (1979). The ecology of human development: Experiments by nature and design. Cambridge, MA: Harvard University Press. ISBN 0-674-22457-4.

Bronfenbrenner, Urie (2005). Making human beings human: Bioecological perspectives on human development. Thousand Oaks, CA: Sage Publications.

Brown, C. S., Mistry, R. S., \& Bigler, R. S. (2007). Hurricane Katrina: African American children's perceptions of race, class, and government involvement amid a national crisis. Analyses of Social Issues and Public Policy, 7, 191-208.doi:10.1111/j.1530-2415.2007.00139.x

Brown, R. C., Witt, A., Fegert, J. M., Keller, F., Rassenhofer, M., \& Plener, P. L. (2017). Psychosocial interventions for children and adolescents after man-made and natural disasters: a meta-analysis and systematic review. Psychological Medicine, 47, 1893-1905. doi:10.1017/s0033291717000496

Bulut, S. (2006). Comparing the earthquake exposed and non-exposed Turkish children's Post Traumatic Stress Reactions. Anales de Psicología/Annals of Psychology, 22, 29-36.

Bulut, S. (2013). Prediction of post-traumatic stress symptoms via comorbid disorders and other social and school problems in earthquake exposed Turkish adolescents. Revista Latinoamericana de Psicología, 45, 47-61.

Bulut, S., Bulut, S., \& Tayli, A. (2005). The dose of exposure and prevalence rates of post traumatic stress disorder in a sample of Turkish children eleven months after the 1999 Marmara earthquakes. School Psychology International, 26, 55-70. doi.org/10.1177/0143034305050893 
Burke jr, J. D., Moccia, P., Borus, J. F., \& Burns, B. J. (1986). Emotional distress in fifth-grade children ten months after a natural disaster. Journal of the American Academy of Child Psychiatry, 25, 536-541. doi:10.1016/S0002-7138(10)60014-3

Burke, J. D., Borus, J. F., Burns, B. J., Millstein, K. H., \& Beasley, M. C. (1982). Changes in children's behavior after a natural disaster. The American Journal of Psychiatry, 139, 1010-1014. doi:10.1176/ajp.139.8.1010

Burnham, J. J., Hooper, L. M., Edwards, E. E., Tippey, J. M., McRaney, A. C., Morrison, M. A., ... \& Woodroof, E. K. (2008). Examining children's fears in the aftermath of hurricane Katrina. Journal of Psychological Trauma, 7, 253-275. doi:10.1080/19322880802492229

Buthmann, J., Finik, J., Ventura, G., Zhang, W., Shereen, A. D., \& Nomura, Y. (2019). The children of Superstorm Sandy: Maternal prenatal depression blunts offspring electrodermal activity. Biological Psychology, 146, Article 107716. https://doi.org/10.1016/j.biopsycho.2019.107716

Buthmann, J., Ham, J., Davey, K., Finik, J., Dana, K., Pehme, P., ... Nomura, Y. (2018). Infant Temperament: Repercussions of Superstorm Sandy-Related Maternal Stress. Child Psychiatry \& Human Development, 50, 150-162. doi:10.1007/s10578-018-0828-2

Cadamuro, A., Versari, A., Vezzali, L., \& Trifiletti, E. (2016). Preventing the detrimental effect of posttraumatic stress in young children: The role of theory of mind in the aftermath of a natural disaster. European Journal of Developmental Psychology, 13, 52-66. doi:10.1080/17405629.2015.1055240

Cadamuro, A., Versari, A., Vezzali, L., Giovannini, D., \& Trifiletti, E. (2015, February). Cognitive performance in the aftermath of a natural disaster: The role of coping strategies, theory of mind and peer social support. Child \& Youth Care Forum, 44, 93-113. doi:10.1007/s10566-014-9272Z 
Cao, X., Laplante, D. P., Brunet, A., Ciampi, A., \& King, S. (2014). Prenatal maternal stress affects motor function in 51/2-year-old children: Project Ice Storm. Developmental psychobiology, 56, 117-125.doi:10.1002/dev.21085

Cartwright, C., Hall, M., \& Lee, A. C. K. (2017). The changing health priorities of earthquake response and implications for preparedness: A scoping review. Public Health, 150, 60-70. doi:10.1016/j.puhe.2017.04.024

Catani, C., Gewirtz, A. H., Wieling, E., Schauer, E., Elbert, T., \& Neuner, F. (2010). Tsunami, war, and cumulative risk in the lives of Sri Lankan schoolchildren. Child Development, 81, 1176-1191. doi: $10.1111 / \mathrm{j} .1467-8624.2010 .01461 . x$

Catani, C., Kohiladevy, M., Ruf, M., Schauer, E., Elbert, T., \& Neuner, F. (2009). Treating children traumatized by war and Tsunami: A comparison between exposure therapy and meditationrelaxation in North-East Sri Lanka. BMC Psychiatry, 9. doi:10.1186/1471-244x-9-22

Celebi Oncu, E., \& Metindogan Wise, A. (2010). The effects of the 1999 Turkish earthquake on young children: Analyzing traumatized children's completion of short stories. Child Development, 81, 1161-1175. doi:10.1111/j.1467-8624.2010.01460.x

Celebi Oncu, E., Akman, B., Guler, T., \& Karaaslan, T. (2009). A report on traumatised and nontraumatised children's human figure drawings reflecting emotional effects of disastrous conditions. Australasian Journal of Trauma Studies, $1 . \quad \mathrm{http}: / / \mathrm{www} . \mathrm{massey}$. ac.nz/ trauma/issues/2009-1/oncu.htm

Cénat, J. M., \& Derivois, D. (2015). Long-term outcomes among child and adolescent survivors of the 2010 Haitian earthquake. Depression and Anxiety, 32, 57-63. doi:10.1002/da.22275. 
Chemtob, C. M., Nakashima, J., \& Carlson, J. G. (2001). Brief treatment for elementary school children with disaster-related posttraumatic stress disorder: A field study. Journal of Clinical Psychology, 58, 99-112. doi:10.1002/jclp.1131

Chen, J., \& Wu, X. (2017). Posttraumatic Stress Symptoms and Posttraumatic Growth in Children and Adolescents Following an Earthquake: A Latent Transition Analysis. Journal of Traumatic Stress, 30, 583-592. doi:10.1002/jts.22238

Chen, W., Wang, L., Zhang, X. L., \& Shi, J. N. (2012). Understanding the impact of trauma exposure on posttraumatic stress symptomatology: A structural equation modeling approach. Journal of Loss and Trauma, 17, 98-110. doi:10.1080/15325024.2011.595293

Cheng, J., Liang, Y., Fu, L., \& Liu, Z. (2018) Posttraumatic stress and depressive symptoms in children after the Wenchuan earthquake. European Journal of Psychotraumatology, 9, 1472992. doi:10.1080/20008198.2018.1472992

Cheng, J., Liang, Y.-M., Zhou, Y.-Y., Eli, B., \& Liu, Z.-K. (2019). Trajectories of PTSD symptoms among children who survived the Lushan earthquake: a four-year longitudinal study. Journal of Affective Disorders, 252, 421-427. doi:10.1016/j.jad.2019.04.047

Cobham, V. E., \& McDermott, B. (2014). Perceived parenting change and child posttraumatic stress following a natural disaster. Journal of Child and Adolescent Psychopharmacology, 24, 18-23. doi:10.1089/cap.2013.0051

Cryder, C. H., Kilmer, R. P., Tedeschi, R. G., \& Calhoun, L. G. (2006). An exploratory study of posttraumatic growth in children following a natural disaster. American Journal of Orthopsychiatry, 76, 65-69. doi:10.1037/0002-9432.76.1.65 
Danielson, C. K., Cohen, J. R., Adams, Z. W., Youngstrom, E. A., Soltis, K., Amstadter, A. B., \& Ruggiero, K. J. (2016). Clinical Decision-Making Following Disasters: Efficient Identification of PTSD Risk in Adolescents. Journal of Abnormal Child Psychology, 45, 117-129.

Danzi, B. A., \& La Greca, A. M. (2017). Optimizing clinical thresholds for PTSD: Extending the DSM-5 preschool criteria to school-age children. International Journal of Clinical and Health Psychology, 17, 234. 241. doi:10.1016/j.ijchp.2017.07.001

Dass-Brailsford, P., Thomley, R. S. H., Talisman, N. W., \& Unverferth, K. (2015). Psychological effects of the 2010 Haitian earthquake on children: An exploratory study. Traumatology, 21, 1421. doi: $10.1037 / \operatorname{trm} 0000015$

Dawson, K. S., Joscelyne, A., Meijer, C., Tampubolon, A., Steel, Z., \& Bryant, R. A. (2014). Predictors of chronic posttraumatic response in Muslim children following natural disaster. Psychological Trauma: Theory, Research, Practice, and Policy, 6, 580-587. doi: $10.1037 / \mathrm{a} 0037140$

Delamater, A. M., \& Applegate, E. B. (1999). Child development and post-traumatic stress disorder after hurricane exposure. Traumatology, 5, 20-27. doi:10.1177/153476569900500303

Demir, T., Demir, D. E., Alkas, L., Copur, M., Dogangun, B., \& Kayaalp, L. (2010). Some clinical characteristics of children who survived the Marmara earthquakes. European Child \& Adolescent Psychiatry, 19, 125-133. doi:10.1007/s00787-009-0048-1

Derivois, D., Cénat, J. M., Joseph, N. E., Karray, A., \& Chahraoui, K. (2017). Prevalence and determinants of post-traumatic stress disorder, anxiety and depression symptoms in street children survivors of the 2010 earthquake in Haiti, four years after. Child Abuse \& Neglect, 67, 174-181. doi:10.1016/j.chiabu.2017.02.034 
Derivois, D., Mérisier, G. G., Cenat, J. M., \& Castelot, V. (2014). Symptoms of posttraumatic stress disorder and social support among children and adolescents after the 2010 Haitian earthquake. Journal of Loss and Trauma, 19, 202-212.doi:10.1080/15325024.2013.789759

Dollinger, S. J. (1985). Lightning-strike disaster among children. British Journal of Medical Psychology, 58, 375-383. doi:10.1111/j.2044-8341.1985.tb02656.x

Dollinger, S. J. (1986). The measurement of children's sleep disturbances and somatic complaints following a disaster. Child Psychiatry and Human Development, 16, 148-153. doi:10.1007/BF00706172

Dollinger, S. J., O'donnell, J. P., \& Staley, A. A. (1984). Lightning-strike disaster: Effects on children's fears and worries. Journal of Consulting and Clinical Psychology, 52, 1028-1038. doi:10.1037/0022-006X.52.6.1028

Drury, S. S., Scheeringa, M. S., \& Zeanah, C. H. (2008). The traumatic impact of hurricane Katrina on children in New Orleans. Child and Adolescent Psychiatric Clinics of North America, 17, 685702. doi:10.1016/j.chc.2008.02.005

Durkin, M. S., Khan, N., Davidson, L. L., Zaman, S. S., \& Stein, Z. A. (1993). The effects of a natural disaster on child behavior: Evidence for posttraumatic stress. American Journal of Public Health, 83, 1549-1553. doi:10.2105/AJPH.83.11.1549

Dyb, G., Jensen, T. K., \& Nygaard, E. (2011). Children's and parents' posttraumatic stress reactions after the 2004 tsunami. Clinical Child Psychology and Psychiatry, 16, 621-634. doi: $10.1177 / 1359104510391048$

Earls, F., Smith, E., Reich, W., \& Jung, K. G. (1988). Investigating psychopathological consequences of a disaster in children: a pilot study incorporating a structured diagnostic interview. Journal of 
RESILIENCE IN CHILDREN AFTER NATURAL DISASTERS

the American Academy of Child \& Adolescent Psychiatry, 27, 90-95. doi:10.1097/00004583198801000-00014

Ekşi, A., Braun, K. L., Ertem-Vehid, H., Peykerli, G., Saydam, R., Toparlak, D., \& Alyanak, B. (2007). Risk factors for the development of PTSD and depression among child and adolescent victims following a 7.4 magnitude earthquake. International Journal of Psychiatry in Clinical Practice, 11, 190-199. doi:10.1080/13651500601017548

Endo, T., Shioiri, T., Someya, T., Toyabe, S., \& Akazawa, K. (2007). Parental mental health affects behavioral changes in children following a devastating disaster: a community survey after the 2004 Niigata-Chuetsu earthquake. General Hospital Psychiatry, 29, 175-176. doi:10.1016/j.genhosppsych.2006.09.006

Evans, L. G., \& Oehler-Stinnett, J. (2006). Structure and prevalence of PTSD symptomology in children who have experienced a severe tornado. Psychology in the Schools, 43, 283-295. doi:10.1002/pits.20150

Evans, L. G., \& Oehler-Stinnett, J. (2008). Validity of the osu post-traumatic stress disorder scale and the behavior assessment system for children self-report of personality with child tornado survivors. Psychology in the Schools, 45, 121-131. doi:10.1002/pits.20285.

Exenberger, S., Ramalingam, P., \& Höfer, S. (2016). Exploring posttraumatic growth in Tamil children affected by the Indian Ocean Tsunami in 2004. International Journal of Psychology: Journal International de Psychologie, 53, 397-401. doi.org/10.1002/ijop.12395. 


\section{RESILIENCE IN CHILDREN AFTER NATURAL DISASTERS}

Exenberger, S., Riedl, D., Rangaramanujam, K., Amirtharaj, V., \& Juen, F. (2019). A cross-sectional study of mother-child agreement on PTSD symptoms in a south Indian post-tsunami sample. $B M C$ Psychiatry, 19, e414. doi:10.1186/s12888-019-2408-9

Fan, F., Long, K., Zhou, Y., Zheng, Y., \& Liu, X. (2015). Longitudinal trajectories of post-traumatic stress disorder symptoms among adolescents after the Wenchuan earthquake in China. Psychological Medicine, 45, 2885-2896. doi:10.1017/S0033291715000884

Farooqui, M., Quadri, S. A., Suriya, S. S., Khan, M. A., Ovais, M., Sohail, Z., ... Hassan, M. (2017). Posttraumatic stress disorder: a serious post-earthquake complication. Trends in Psychiatry and Psychotherapy, 39, 135-143. doi:10.1590/2237-6089-2016-0029

Felix, E. D., You, S. K., \& Canino, G. (2013). School and community influences on the long term postdisaster recovery of children and youth following hurricane Georges. Journal of Community Psychology, 41, 1021-1038. doi.org/10.1002/jcop.21590

Felix, E. D., You, S., \& Canino, G. (2015). Family influences on the relationship between hurricane exposure and ataques de nervios. Journal of Child and Family Studies, 24, 2229-2240. doi:10.1007/s10826-014-0025-2

Felix, E., Hernández, L. A., Bravo, M., Ramirez, R., Cabiya, J., \& Canino, G. (2011). Natural disaster and risk of psychiatric disorders in Puerto Rican children. Journal of Abnormal Child Psychology, 39, 589-600. doi:10.1007/s10802-010-9483-1

Felix, E., Kaniasty, K., You, S., \& Canino, G. (2015). Parent-child relationship quality and gender as moderators of the influence of hurricane exposure on physical health among children and youth. Journal of Pediatric Psychology, 41, 73-85. doi:10.1093/jpepsy/jsv038. 
Felix, E., You, S., Vernberg, E., \& Canino, G. (2013). Family influences on the long term postdisaster recovery of Puerto Rican youth. Journal of Abnormal Child Psychology, 41, 111-124. doi:10.1007/s10802-012-9654-3

Felton, J. W., Cole, D. A., \& Martin, N. C. (2013). Effects of rumination on child and adolescent depressive reactions to a natural disaster: The 2010 Nashville flood. Journal of Abnormal Psychology, 122, 64-73. doi:10.1037/a0029303

Feo, P., Di Gioia, S., Carloni, E., Vitiello, B., Tozzi, A. E., \& Vicari, S. (2014). Prevalence of psychiatric symptoms in children and adolescents one year after the 2009 L'Aquila earthquake. BMC Psychiatry, 14, 270. doi:10.1186/s12888-014-0270-3

Fernandez, I. (2007). EMDR as a treatment of post-traumatic reactions: A field study on child victims of an earthquake. Educational and Child Psychology, 24, 65-72.

Field, T., Seligman, S., Scafidi, F., \& Schanberg, S. (1996). Alleviating posttraumatic stress in children following hurricane Andrew. Journal of Applied Developmental Psychology, 17, 37-50. doi:10.1016/s0193-3973(96)90004-0

Fivush, R., McDermott Sales, J., Goldberg, A., Bahrick, L., \& Parker, J. (2004). Weathering the storm: Children's long-term recall of hurricane Andrew. Memory, 12, 104-118. doi: $10.1080 / 09658210244000397$

Foa, E. B., Johnson, K. M., Feeny, N. C., \& Treadwell, K. R. (2001). The Child PTSD Symptom Scale: A preliminary examination of its psychometric properties. Journal of Clinical Child Psychology, 30, 376-384. doi:10.1207/S15374424JCCP3003_9 
RESILIENCE IN CHILDREN AFTER NATURAL DISASTERS

Forman-Hoffman, V. L., Zolotor, A. J., McKeeman, J. L., Blanco, R., Knauer, S. R., Lloyd, S. W., \& Viswanathan, M. (2013). Comparative effectiveness of interventions for children exposed to nonrelational traumatic events. Pediatrics, 131, 526-539. doi:10.1542/peds.2012-3846

Fu, C., \& Underwood, C. (2015). A meta-review of school-based disaster interventions for child and adolescent survivors. Journal of Child \& Adolescent Mental Health, 27, 161-171. doi:10.2989/17280583.2015.1117978

Fu, C., Leoutsakos, J.-M., \& Underwood, C. (2013). Moderating effects of a postdisaster intervention on risk and resilience factors associated with Posttraumatic Stress Disorder in Chinese children. Journal of Traumatic Stress, 26, 663-670. doi:10.1002/jts.21871

Fujiwara, T., Mizuki, R., Miki, T., \& Chemtob, C. (2015). Association between facial expression and PTSD symptoms among young children exposed to the Great East Japan earthquake: a pilot study. Frontiers in Psychology, 6, 1534. doi:10.3389/fpsyg.2015.01534.

Fujiwara, T., Yagi, J., Homma, H., Mashiko, H., Nagao, K., \& Okuyama, M. (2017). Symptoms of post-traumatic stress disorder among young children 2 years after the Great East Japan earthquake. Disaster Medicine and Public Health Preparedness, 11, 207-215. doi:10.1017/dmp.2016.101

Fujiwara, T., Yagi, J., Homma, H., Mashiko, H., Nagao, K., Okuyama, M., ... \& Children Study Team. (2017). Suicide risk among young children after the Great East Japan earthquake: A followup study. Psychiatry Research, 253, 318-324. doi:10.1016/j.psychres.2017.04.018

Furr, J. M., Comer, J. S., Edmunds, J. M., \& Kendall, P. C. (2010). Disasters and youth: A metaanalytic examination of posttraumatic stress. Journal of Consulting and Clinical Psychology, 78, 765-780. doi:10.1037/a0021482 
Galante, R., \& Foa, D. (1986). An epidemiological study of psychic. Annual Progress in Child Psychiatry and Child Development, 25, 357-363. doi:10.1192/S0007125000175208

Garfin, D. R., Silver, R. C., Gil-Rivas, V., Guzmán, J., Murphy, J. M., Cova, F., ... Guzmán, M. P. (2014). Children's reactions to the 2010 Chilean earthquake: The role of trauma exposure, family context, and school-based mental health programming. Psychological Trauma: Theory, Research, Practice, and Policy, 6, 563-573. doi:10.1037/a0036584

Garrison, C. Z., Bryant, E. S., Addy, C. L., Spurrier, P. G., Freedy, J. R., \& Kilpatrick, D. G. (1995). Posttraumatic stress disorder in adolescents after hurricane Andrew. Journal of the American Academy of Child \& Adolescent Psychiatry, 34, 1193-1201. doi:10.1097/00004583-199509000$\underline{00017}$

Ge, F., Yuan, M., Li, Y., Zhang, J., \& Zhang, W. (2019). Changes in the network structure of posttraumatic stress disorder symptoms at different time points among youth survivors: A network analysis. Journal of Affective Disorders, 259, 288-295. https://doi.org/10.1016/j.jad.2019.08.065

Giannopoulou, I., Dikaiakou, A., \& Yule, W. (2006). Cognitive-behavioural group intervention for PTSD Symptoms in children following the Athens 1999 earthquake: A pilot study. Clinical Child Psychology and Psychiatry, 11, 543-553. doi:10.1177/1359104506067876

Giannopoulou, I., Smith, P., Ecker, C., Strouthos, M., Dikaiakou, A., \& Yule, W. (2006). Factor structure of the Children's Revised Impact of Event Scale (CRIES) with children exposed to earthquake. Personality and Individual Differences, 40, 1027-1037. doi:10.1016/j.paid.2005.11.002 


\section{RESILIENCE IN CHILDREN AFTER NATURAL DISASTERS}

Giannopoulou, I., Strouthos, M., Smith, P., Dikaiakou, A., Galanopoulou, V., \& Yule, W. (2006). Post-traumatic stress reactions of children and adolescents exposed to the Athens 1999 earthquake. European Psychiatry, 21, 160-166. doi:10.1016/j.eurpsy.2005.09.005

Gil-Rivas, V., \& Kilmer, R. P. (2013). Children's adjustment following Hurricane Katrina: the role of primary caregivers. American Journal of Orthopsychiatry, 83,413-421. doi:10.1111/ajop.12016

Goenjian, A. (1993). A mental health relief programme in Armenia after the 1988 earthquake. British Journal of Psychiatry, 163, 230-239. doi:10.1192/bjp.163.2.230

Goenjian, A. K., Pynoos, R. S., Steinberg, A. M., Najarian, L. M., Asarnow, J. R., Karayan, I., ... \& Fairbanks, L. A. (1995). Psychiatric comorbidity in children after the 1988: Earthquake in Armenia. Journal of the American Academy of Child \& Adolescent Psychiatry, 34, 1174-1184. doi:10.1097/00004583-199509000-00015

Goenjian, A. K., Walling, D., Steinberg, A. M., Roussos, A., Goenjian, H. A., \& Pynoos, R. S. (2009). Depression and PTSD symptoms among bereaved adolescents $6 \frac{1}{2}$ years after the 1988 spitak earthquake. Journal of Affective Disorders, 112, 81-84. doi:10.1016/j.jad.2008.04.006

Gökçen, C., Şahingöz, M., \& Annagür, B. B. (2013). Does a non-destructive earthquake cause posttraumatic stress disorder? A cross-sectional study. European Child \& Adolescent Psychiatry, 22, 295-299. doi:10.1007/s00787-012-0348-8

Gomez, C. J., \& Yoshikawa, H. (2017). Earthquake effects: Estimating the relationship between exposure to the 2010 Chilean earthquake and preschool children's early cognitive and executive function skills. Early Childhood Research Quarterly, 38, 127-136. doi:10.1016/j.ecresq.2016.08.004 
RESILIENCE IN CHILDREN AFTER NATURAL DISASTERS

Graham, R. A., Osofsky, J. D., Osofsky, H. J., \& Hansel, T. C. (2017). School based post disaster mental health services: Decreased trauma symptoms in youth with multiple traumas. Advances in School Mental Health Promotion, 10, 161-175. doi:10.1080/1754730x.2017.1311798

Green, B. L., Korol, M., Grace, M. C., Vary, M. G., Leonard, A. C., Gleser, G. C., \& Smitson-Cohen, S. (1991). Children and disaster: Age, gender, and parental effects on PTSD symptoms. Journal of the American Academy of Child \& Adolescent Psychiatry, 30, 945-951. doi:10.1097/00004583199111000-00012

Groome, D., \& Soureti, A. (2004). Post-traumatic stress disorder and anxiety symptoms in children exposed to the 1999 Greek earthquake. British Journal of Psychology, 95, 387-397. doi: $10.1348 / 0007126041528149$

Hafstad, G. S., Gil-Rivas, V., Kilmer, R. P., \& Raeder, S. (2010). Parental adjustment, family functioning, and posttraumatic growth among Norwegian children and adolescents following a natural disaster. American Journal of Orthopsychiatry, 80, 248-257. doi:10.1111/j.19390025.2010 .0102

Hafstad, G. S., Kilmer, R. P., \& Gil-Rivas, V. (2011). Posttraumatic growth among Norwegian children and adolescents exposed to the 2004 tsunami. Psychological Trauma: Theory, Research, Practice, and Policy, 3, 130-138. doi:10.1037/a0023236

Hall, A., \& Kirby, H. (2010). The numbers, educational status and health of enrolled and non-enrolled school-age children in the Allai Valley, Northwest Frontier Province, Pakistan. Social Science \& Medicine, 70, 1131-1140. doi:10.1016/j.socscimed.2009.12.021

Hambrick, E. P., O'connor, B. M., \& Vernberg, E. M. (2016). Interview and recollection-based research with child disaster survivors: Participation-related changes in emotion and perceptions of 
RESILIENCE IN CHILDREN AFTER NATURAL DISASTERS

participation. Psychological Trauma: theory, research, practice, and policy, 8, 165-171. doi: $10.1037 /$ tra0000071

Hambrick, E. P., Vernberg, E. M., Greenhoot, A. F., \& Hendrickson, M. L. (2017). Recalling a Devastating Tornado: Qualities of Child Recollections and Associations with Mental Health Symptoms. Journal of Child \& Adolescent Trauma, 11, 227-239. doi:10.1007/s40653-017-0199Z

Han, L., Zhang, Y., \& Zheng, Y. (2012). Responses over time of child and adolescent survivors to the 2008 Wenchuan, China earthquake. Social Behavior and Personality: an international journal, 40, 1147-1152. doi:10.2224/sbp.2012.40.7.1147

Hansel, T. C., Osofsky, H. J., Osofsky, J. D., \& Speier, A. H. (2019). Katrina inspired disaster screenings and services: School-based trauma interventions. Traumatology, 25, 133-141. https://doi.org/10.1037/trm0000178

Hansel, T. C., Osofsky, J. D., Osofsky, H. J., \& Friedrich, P. (2013). The effect of long-term relocation on child and adolescent survivors of hurricane Katrina. Journal of Traumatic Stress, 26, 613-620. doi.org/10.1002/jts.21837

Hausman, E. M., Black, S. R., Bromet, E., Carlson, G., Danzig, A., Kotov, R., \& Klein, D. N. (2020). Reciprocal effects of maternal and child internalizing symptoms before and after a natural disaster. Journal of family psychology. Advance online publication. https://doi.org/10.1037/fam0000653

Hensley-Maloney, L., \& Varela, R. E. (2009). The influence of hurricane exposure and anxiety sensitivity on panic symptoms. Child and Youth Care Forum, 38, 135-149. doi:10.1007/s10566009-9072-z 
RESILIENCE IN CHILDREN AFTER NATURAL DISASTERS

Hizli, F. G., Taskintuna, N., Isikli, S., Kilic, C., \& Zileli, L. (2009). Predictors of posttraumatic stress in children and adolescents. Children and Youth Services Review, 31, 349-354. doi:10.1016/j.childyouth.2008.08.008.

Hlodversdottir, H., Thorsteinsdottir, H., Thordardottir, E. B., Njardvik, U., Petursdottir, G., \& Hauksdottir, A. (2018). Long-term health of children following the Eyjafjallajökull volcanic eruption: a prospective cohort study. European Journal of Psychotraumatology, 9 (sup2), 1442601

Ho, R. T. H., Lai, A. H. Y., Lo, P. H. Y., Nan, J. K. M., \& Pon, A. K. L. (2016). A strength-based arts and play support program for young survivors in post-quake china: effects on self-efficacy, peer support, and anxiety. The Journal of Early Adolescence, 37, 805-824. doi: $10.1177 / 0272431615624563$

Houlihan, D., Ries, B. J., Polusny, M. A., \& Hanson, C. N. (2008). Predictors of behavior and level of life satisfaction of children and adolescents after a major tornado. Journal of Psychological Trauma, 7, 21-36.

Hsu, C. C., Chong, M. Y., Yang, P., \& Yen, C. F. (2002). Posttraumatic stress disorder among adolescent earthquake victims in Taiwan. Journal of the American Academy of Child \& Adolescent Psychiatry, 41, 875-881. doi:10.1097/00004583-200207000-00022

Huzziff, C. A., \& Ronan, K. R. (1999). Prediction of children's coping following a natural disasterthe Mount Ruapehu eruptions: A prospective study. Australasian Journal of Disaster and Trauma Studies, 1, 1999-1. http://massey.ac.nz/ trauma/

Itagaki, S., Harigane, M., Maeda, M., Yasumura, S., Suzuki, Y., Mashiko, H., ... \& Mental Health Group of the Fukushima Health Management Survey. (2017). Exercise habits are important for the mental health of children in Fukushima after the Fukushima Daiichi disaster: The Fukushima 
RESILIENCE IN CHILDREN AFTER NATURAL DISASTERS

Health Management Survey. Asia Pacific Journal of Public Health, 29, 171-181. doi: $10.1177 / 1010539516686163$

Iwadare, Y., Usami, M., Suzuki, Y., Ushijima, H., Tanaka, T., Watanabe, K., ... \& Saito, K. (2014). Posttraumatic symptoms in elementary and junior high school children after the 2011 Japan earthquake and tsunami: Symptom severity and recovery vary by age and sex. The Journal of Pediatrics, 164, 917-921. doi: 10.1016/j.jpeds.2013.11.061

Jackson, S. F., Fazal, N., Gravel, G., \& Papowitz, H. (2017). Evidence for the value of health promotion interventions in natural disaster management. Health Promotion International, 32, 1057-1066. doi:10.1093/heapro/daw029

Jarero, I., Artigas, L., \& Hartung, J. (2006). EMDR integrative group treatment protocol: A postdisaster trauma intervention for children and adults. Traumatology, 12, 121-129. doi: $10.1177 / 1534765606294561$

Jeney-Gammon, P., Daugherty, T. K., Finch Jr, A. J., Belter, R. W., \& Foster, K. Y. (1993). Children's coping styles and report of depressive symptoms following a natural disaster. The Journal of Genetic Psychology, 154, 259-267. doi:10.1080/00221325.1993.9914739

Jensen, T. K., Ellestad, A., \& Dyb, G. (2013). Children and adolescents' self-reported coping strategies during the Southeast Asian Tsunami. British Journal of Clinical Psychology, 52, 92106. doi:10.1111/bjc. 12003 
RESILIENCE IN CHILDREN AFTER NATURAL DISASTERS

Jia, Z., Tian, W., He, X., Liu, W., Jin, C., \& Ding, H. (2010). Mental health and quality of life survey among child survivors of the 2008 Sichuan earthquake. Quality of Life Research, 19, 1381-1391. doi:10.1007/s11136-010-9703-8

Jieling, C., \& Xinchun, W. (2017). Post-traumatic stress symptoms and post-traumatic growth among children and adolescents following an earthquake: A latent profile analysis. Child and Adolescent Mental Health, 22, 23-29. doi:10.1111/camh.12175

Jones, R. T., Frary, R., Cunningham, P., Weddle, J. D., \& Kaiser, L. (2001). The psychological effects of Hurricane Andrew on ethnic minority and Caucasian children and adolescents: A case study. Cultural Diversity and Ethnic Minority Psychology, 7, 103. doi:10.1037/1099-9809.7.1.103

Jurgens, J. J., Houlihan, D., \& Schwartz, C. (1996). Behavioral manifestations of adolescent school relocation and trauma. Child \& family behavior therapy, 18, 1-8. doi:10.1300/J019v18n01_01

Juth, V., Silver, R. C., Seyle, D. C., Widyatmoko, C. S., \& Tan, E. T. (2015). Post-disaster mental health among parent-child dyads after a major earthquake in Indonesia. Journal of Abnormal Child Psychology, 43, 1309-1318. doi:10.1080/01650250042000113

Kalantari, M., \& Vostanis, P. (2010). Behavioural and emotional problems in Iranian children four years after parental death in an earthquake. International Journal of Social Psychiatry, 56, 158167. doi:10.1177/0020764008101854

Kar, N. (2011). Cognitive behavioral therapy for the treatment of post-traumatic stress disorder: A review. Neuropsychiatric Disease and Treatment, 7, 167-181. doi:10.2147/ndt.s10389

Kar, N., Mohapatra, P. K., Nayak, K. C., Pattanaik, P., Swain, S. P., \& Kar, H. C. (2007). Posttraumatic stress disorder in children and adolescents one year after a super-cyclone in Orissa, India: 
RESILIENCE IN CHILDREN AFTER NATURAL DISASTERS

Exploring cross-cultural validity and vulnerability factors. BMC Psychiatry, 7, 8. doi:10.1186/1471-244X-7-8

Karairmak, Ö., \& Aydin, G. (2008). Brief report: Reducing earthquake-related fears in victim and nonvictim children. The Journal of Genetic Psychology, 169, 177-186. doi:10.3200/gntp.169.2.177-186

Kelley, M. L., Self-Brown, S., Le, B., Bosson, J. V., Hernandez, B. C., \& Gordon, A. T. (2010). Predicting posttraumatic stress symptoms in children following Hurricane Katrina: A prospective analysis of the effect of parental distress and parenting practices. Journal of Traumatic Stress, 23, 582-590. doi:10.1002/jts.20573

Kessel, E. M., Nelson, B. D., Finsaas, M., Kujawa, A., Meyer, A., Bromet, E., ... Klein, D. N. (2019). Parenting style moderates the effects of exposure to natural disaster-related stress on the neural development of reactivity to threat and reward in children. Development and Psychopathology, 110. doi: $10.1017 / \mathrm{s} 0954579418001347$

Kessel, E. M., Nelson, B. D., Kujawa, A., Hajcak, G., Kotov, R., Bromet, E. J., ... \& Klein, D. N. (2018). Hurricane Sandy exposure alters the development of neural reactivity to negative stimuli in children. Child Development, 89, 339-348. doi:10.1111/cdev.12691

Kiliç, C., Kiliç, E. Z., \& Aydin, I. O. (2011). Effect of relocation and parental psychopathology on earthquake survivor-children's mental health. The Journal of Nervous and Mental Disease, 199, 335-341. doi:10.1097/NMD.0b013e3182174ffa

Kiliç, E. Z., Kiliç, C., \& Yılmaz, S. (2008). Is anxiety sensitivity a predictor of PTSD in children and $\begin{array}{lllll}\text { adolescents? } & \text { Journal } & \text { 8f }\end{array}$ doi:10.1016/j.jpsychores.2008.02.013 
Kiliç, E. Z., Özgüven, H. D., \& Sayil, I. (2003). The psychological effects of parental mental health on children experiencing disaster: The experience of Bolu earthquake in Turkey. Family Process, 42, 485-495. doi:10.1111/j.1545-5300.2003.00485.x

King, L. S., Osofsky, J. D., Osofsky, H. J., Weems, C. F., Hansel, T. C., \& Fassnacht, G. M. (2015). Perceptions of trauma and loss among children and adolescents exposed to disasters a mixedmethods study. Current Psychology, 34, 524-536. doi.org/10.1007/s12144-015-9348-4

King, S., \& Laplante, D. P. (2005). The effects of prenatal maternal stress on children's cognitive development: Project Ice Storm. Stress, 8, 35-45. doi 10.1080/10253890500108391

Kiser, L., Heston, J., Hickerson, S., Millsap, P., Nunn, W., \& Pruitt, D. (1993). Anticipatory stress in children and adolescents. American Journal of Psychiatry, 150, 87-87. doi:10.1176/ajp.150.1.87

Klontz, B. T., Bivens, A., Michels, S., DeLeon, P. H., \& Tom, L. (2015). The Mokihana Program: The effectiveness of an integrated department of education and department of health school-based behavioral health approach. Psychological Services, 12, 101-111. doi:10.1037/a0038015

Knight, L. A., \& Sullivan, M. A. (2006). Preliminary development of a measure to assess children's trauma attributions. Journal of Aggression, Maltreatment \& Trauma, 13, 65-78. doi.org/10.1300/J146v13n02_05

Kolaitis, G., Kotsopoulos, J., Tsiantis, J., Haritaki, S., Rigizou, F., Zacharaki, L., ... \& Liakopoulou, M. (2003). Posttraumatic stress reactions among children following the Athens earthquake of September 1999. European Child \& Adolescent Psychiatry, 12, 273-280. doi.org/10.1007/s00787-003-0339-x

Kopala-Sibley, D. C., Danzig, A. P., Kotov, R., Bromet, E. J., Carlson, G. A., Olino, T. M., ... \& Klein, D. N. (2016). Negative emotionality and its facets moderate the effects of exposure to 
RESILIENCE IN CHILDREN AFTER NATURAL DISASTERS

Hurricane Sandy on children's postdisaster depression and anxiety symptoms. Journal of Abnormal Psychology, 125, 471-481. doi.org/10.1037/abn0000152

Kreuger, L., \& Stretch, J. (2003). Identifying and helping long term child and adolescent disaster victims: Model and method, 30, 93-108. Journal of Social Service Research. doi.org/10.1300/J079v30n02_06

Kronenberg, M. E., Hansel, T. C., Brennan, A. M., Osofsky, H. J., Osofsky, J. D., \& Lawrason, B. (2010). Children of Katrina: Lessons learned about postdisaster symptoms and recovery patterns. Child Development, 81, 1241-1259. doi:10.1111/j.1467-8624.2010.01465.x

Kroska, E. B., O’Hara, M. W., Elgbeili, G., Hart, K. J., Laplante, D. P., Dancause, K. N., \& King, S. (2018). The impact of maternal flood-related stress and social support on offspring weight in early childhood. Archives of Women's Mental Health, 21, 225-233. doi.org/10.1007/s00737-017-0786X

Küçükoğlu, S., Yıldırım, N., \& Dursun, O. B. (2015). Posttraumatic stress symptoms seen in children within the 3-month period after the Van earthquake in Turkey. International Journal of Nursing Practice, 21, 542-549. doi.org/10.1111/ijn.12305

Kujawa, A., Hajcak, G., Danzig, A. P., Black, S. R., Bromet, E. J., Carlson, G. A., ... \& Klein, D. N. (2016). Neural reactivity to emotional stimuli prospectively predicts the impact of a natural disaster on psychiatric symptoms in children. Biological Psychiatry,80, 381-389. doi:10.1016/j.biopsych.2015.09.008

Kumar, M., \& Fonagy, P. (2012). Conceptualizing attachment trauma: Exploring emotional vulnerabilities among disaster affected children of Gujarat. Psychological Studies, 57, 9-21. doi:10.1007/s12646-011-0114-X 
RESILIENCE IN CHILDREN AFTER NATURAL DISASTERS

Kumar, M., \& Fonagy, P. (2013). Differential effects of exposure to social violence and natural disaster on children's mental health. Journal of traumatic stress, 26, 695-702. doi:10.1002/jts. 21874

Kuwabara, H., Araki, T., Yamasaki, S., Ando, S., Kano, Y., \& Kasai, K. (2015). Regional differences in post-traumatic stress symptoms among children after the 2011 tsunami in Higashi-Matsushima, Japan. Brain and Development, 37, 130-136. doi:10.1016/j.braindev.2014.02.003

La Greca, A. M., Danzi, B. A., \& Chan, S. F. (2017). DSM-5 and ICD-11 as competing models of PTSD in preadolescent children exposed to a natural disaster: Assessing validity and co-occurring symptomatology. European Journal of Psychotraumatology, 8, 1310591. doi:10.1080/20008198.2017.1310591

La Greca, A. M., Lai, B. S., Joormann, J., Auslander, B. B., \& Short, M. A. (2013). Children's risk and resilience following a natural disaster: Genetic vulnerability, posttraumatic stress, and depression. Journal of Affective Disorders, 151, 860-867. doi:10.1016/j.jad.2013.07.024

La Greca, A. M., Lai, B. S., Llabre, M. M., Silverman, W. K., Vernberg, E. M., \& Prinstein, M. J. (2013, August). Children's postdisaster trajectories of PTS symptoms: Predicting chronic distress. Child \& Youth Care Forum, 2, 351-369. doi:10.1007/s10566-013-9206-1

La Greca, A. M., Silverman, W. K., \& Wasserstein, S. B. (1998). Children's predisaster functioning as a predictor of posttraumatic stress following Hurricane Andrew. Journal of consulting and clinical psychology, 66, 883-892. doi:10.1037//0022-006X.66.6.883

La Greca, A. M., Silverman, W. K., Lai, B., \& Jaccard, J. (2010). Hurricane-related exposure experiences and stressors, other life events, and social support: Concurrent and prospective impact on children's persistent posttraumatic stress symptoms. Journal of Consulting and Clinical Psychology, 78, 794. doi.org/10.1037/a0020775. 
La Greca, A. M., Silverman, W. K., Vernberg, E. M., \& Prinstein, M. J. (1996). Symptoms of posttraumatic stress in children after Hurricane Andrew: A prospective study. Journal of Consulting and Clinical Psychology, 64, 712-723. doi:10.1037/0022-006X.64.4.712

Lack, C. W., Sullivan, M. A., \& Knight, L. A. (2008). Assessing posttraumatic stress in children: A review and further examination of the psychometrics of Frederick's Reaction Index. Journal of Child \& Adolescent Trauma, 1, 225-232. doi:10.1080/19361520802279141

Lai, B. S., Beaulieu, B., Ogokeh, C. E., Self-Brown, S., \& Kelley, M. L. (2015). Mother and child reports of hurricane related stressors: Data from a sample of families exposed to Hurricane Katrina. Child \& youth Care Forum, 4, 549-565. doi:10.1007/s10566-014-9289-3

Lai, B. S., Kelley, M. L., Harrison, K. M., Thompson, J. E., \& Self-Brown, S. (2015). Posttraumatic stress, anxiety, and depression symptoms among children after Hurricane Katrina: A latent profile analysis. Journal of Child and Family Studies, 24, 1262-1270. doi:10.1007/s10826-014-9934-3

Lai, B. S., La Greca, A. M., \& Llabre, M. M. (2014). Children's sedentary activity after hurricane exposure. Psychological Trauma: Theory, Research, Practice, and Policy, 6, 280-289. doi:10.1037/a0033331

Lai, B. S., La Greca, A. M., Auslander, B. A., \& Short, M. B. (2013). Children's symptoms of posttraumatic stress and depression after a natural disaster: Comorbidity and risk factors. Journal of Affective Disorders, 146, 71-78. doi:10.1016/j.jad.2012.08.041

Lai, B. S., Lewis, R., Livings, M. S., La Greca, A. M., \& Esnard, A.-M. (2017). Posttraumatic stress symptom trajectories among children after disaster exposure: A review. Journal of Traumatic Stress, 30, 571-582. doi:10.1002/jts.22242 
Lai, B. S., Osborne, M. C., Piscitello, J., Self-Brown, S., \& Kelley, M. L. (2018). The relationship between social support and posttraumatic stress symptoms among youth exposed to a natural $\begin{array}{llll}\text { disaster. European } \quad \text { Journal of } & \text { Psychotraumatology, 9, } & 1450042 .\end{array}$ doi:10.1080/20008198.2018.1450042

Lai, B. S., Tiwari, A., Beaulieu, B. A., Self-Brown, S., \& Kelley, M. L. (2015). Hurricane Katrina: Maternal depression trajectories and child outcomes. Current Psychology, 34, 515-523. doi:10.1007/s12144-015-9338-6.

Laor, N., Wolmer, L., Kora, M., Yucel, D., Spirman, S., \& Yazgan, Y. (2002). Posttraumatic, dissociative and grief symptoms in Turkish children exposed to the 1999 earthquakes. The Journal of Nervous and Mental Disease, 190, 824-832. doi:10.1097/01.NMD.0000041959.54021.A7

Laplante, D. P., Brunet, A., Schmitz, N., Ciampi, A., \& King, S. (2008). Project Ice Storm: Prenatal maternal stress affects cognitive and linguistic functioning in 51/2-year-old children. Journal of the American Academy of Child \& Adolescent Psychiatry, 47, 1063-1072. doi:10.1097/CHI.0b013e31817eec80

Laplante, D. P., Zelazo, P. R., Brunet, A., \& King, S. (2007). Functional play at 2 years of age: Effects of prenatal maternal stress. Infancy, 12, 69-93. doi:10.1111/j.1532-7078.2007.tb00234.x

Lee, O. (1999). Science knowledge, world views, and information sources in social and cultural contexts: Making sense after a natural disaster. American Educational Research Journal, 36, 187219. doi: $10.3102 / 00028312036002187$

Li, G., Wang, L., Cao, C., Fang, R., Cao, X., Chen, C., Elhai, J. D., \& Hall, B. J. (2019). Posttraumatic stress disorder and executive dysfunction among children and adolescents: A Latent Profile Analysis. International Journal of Clinical and Health Psychology, 19, 228-236. https://doi.org/10.1016/j.ijchp.2019.07.001 
RESILIENCE IN CHILDREN AFTER NATURAL DISASTERS

Li, X., Huang, X., Tan, H., Liu, A., Zhou, J., \& Yang, T. (2010). A study on the relationship between posttraumatic stress disorder in flood victim parents and children in Hunan, China. Australian and New Zealand Journal of Psychiatry, 44, 543-550. doi:10.3109/00048671003601400

Li, Y., Li, H., Decety, J., \& Lee, K. (2013). Experiencing a natural disaster alters children's altruistic giving. Psychological Science, 24, 1686-1695. doi:10.1177/0956797613479975

Liang, Y., Cheng, J., Zhou, Y., \& Liu, Z. (2019). Trajectories of posttraumatic stress disorders among children after the Wenchuan earthquake: a four-year longitudinal study. European Journal of Psychotraumatology, 10, 1586266. doi:10.1080/20008198.2019.1586266

Liao, T. L., Chen, Y. S., Chen, C. Y., \& Chien, L. Y. (2014). Self-reported internalizing and externalizing behaviours among junior high school students at 2 and 4 years after the 921 earthquake in Taiwan. Stress and Health, 30, 265-271. doi:10.1002/smi.2506

Liu, K., Liang, X., Guo, L., Li, Y., Li, X., Xin, B., ... \& Li, Y. (2010). Acute stress disorder in the paediatric surgical children and adolescents injured during the Wenchuan earthquake in China. Stress and Health, 26, 262-268. doi:10.1002/smi.1288

Liu, M., Wang, L., Shi, Z., Zhang, Z., Zhang, K., \& Shen, J. (2011). Mental health problems among children one-year after Sichuan earthquake in China: A follow-up study. PloS one, 6, e14706. doi:10.1371/journal.pone.0014706

Liu, X., Yang, H., Tang, B., Liu, Y., \& Zhang, L. (2017). Health status of adolescents in the Tibetan plateau area of western China: 6 years after the Yushu earthquake. Health and Quality of Life Outcomes, 15, 152. doi: 10.1186/s12955-017-0727-4

Liu, Z., Zhu, Z., Kao, H. S. R., Zong, Y., Tang, S., Xu, M., ... Wang, R. (2014). Effect of calligraphy training on hyperarousal symptoms for childhood survivors of the 2008 China earthquakes. Neuropsychiatric Disease and Treatment, 10, 977-985. doi:10.2147/ndt.s55016 
Lochman, J. E., Vernberg, E., Powell, N. P., Boxmeyer, C. L., Jarrett, M., McDonald, K., ... \& Kassing, F. (2017). Pre-post tornado effects on aggressive children's psychological and behavioral adjustment through one-year postdisaster. Journal of Clinical Child \& Adolescent Psychology, 46, 136-149. doi:10.1080/15374416.2016.1228460

Lonigan, C. J., Shannon, M. P., Finch Jr, A. J., Daugherty, T. K., \& Taylor, C. M. (1991). Children's reactions to a natural disaster: Symptom severity and degree of exposure. Advances in Behaviour Research and Therapy, 13, 135-154. doi:10.1016/0146-6402(91)90002-R

Lonigan, C. J., Shannon, M. P., Taylor, C. M., Finch Jr, A. J., \& Sallee, F. R. (1994). Children exposed to disaster: II. Risk factors for the development of post-traumatic symptomatology. Journal of the American Academy of Child \& Adolescent Psychiatry, 33, 94-105. doi:10.1097/00004583199401000-00013

Lowe, S. R., Godoy, L., Rhodes, J. E., \& Carter, A. S. (2013). Predicting mothers' reports of children's mental health three years after Hurricane Katrina. Journal of Applied Developmental Psychology, 34, 17-27. doi:10.1016/j.appdev.2012.09.002

Ma, X., Liu, X., Hu, X., Qiu, C., Wang, Y., Huang, Y., ... \& Li, T. (2011). Risk indicators for posttraumatic stress disorder in adolescents exposed to the 5.12 Wenchuan earthquake in China. Psychiatry Research, 189, 385-391. doi:10.1016/j.psychres.2010.12.016

Madkour, A. S., Johnson, C. C., Clum, G. A., \& Brown, L. (2011). Disaster and youth violence: The experience of school-attending youth in New Orleans. Journal of Adolescent Health, 49, 213-215. doi:10.1016/j.jadohealth.2011.06.005 
RESILIENCE IN CHILDREN AFTER NATURAL DISASTERS

Martin, N. C., Felton, J. W., \& Cole, D. A. (2016). Predictors of youths' posttraumatic stress symptoms following a natural disaster: The 2010 Nashville, Tennessee, flood. Journal of Clinical Child \& Adolescent Psychology, 45, 335-347. doi:10.1080/15374416.2014.982279

Mashiko, H., Yabe, H., Maeda, M., Itagaki, S., Kunii, Y., Shiga, T., .. \& Niwa, S. I. (2017). Mental health status of children after the Great East Japan Earthquake and Fukushima Daiichi nuclear power plant accident. Asia Pacific Journal of Public Health,29, 131-138. doi:10.1177/1010539516675702

McDermott, B. M., \& Cobham, V. E. (2012). Family functioning in the aftermath of a natural disaster. BMC Psychiatry, 12, 55. doi:10.1186/1471-244X-12-55

McDermott, B. M., Cobham, V. E., Berry, H., \& Stallman, H. M. (2010). Vulnerability factors for disaster-induced child post-traumatic stress disorder: The case for low family resilience and previous mental illness. Australian and New Zealand Journal of Psychiatry, 44, 384-389. doi: $10.3109 / 00048670903489916$

McDermott, B., Berry, H., \& Cobham, V. (2012). Social connectedness: A potential aetiological factor in the development of child post-traumatic stress disorder. Australian \& New Zealand Journal of Psychiatry, 46, 109-117. doi:10.1177/0004867411433950

McDermott, B., Cobham, V., Berry, H., \& Kim, B. (2014). Correlates of persisting posttraumatic symptoms in children and adolescents 18 months after a cyclone disaster. Australian \& New Zealand Journal of Psychiatry, 48, 80-86. doi:10.1177/0004867413500349

McDonald, K. L., Vernberg, E. M., Lochman, J. E., Abel, M. R., Jarrett, M. A., Kassing, F., Powell, N., \& Qu, L. (2019). Trajectories of tornado-related posttraumatic stress symptoms and preexposure predictors in a sample of at-risk youth. Journal of Consulting and Clinical Psychology, 87, 1003-1018. https://doi.org/10.1037/ccp0000432 
McFarlane, A. C. (1987). The relationship between patterns of family interaction and psychiatric disorder in children. Australian \& New Zealand Journal of Psychiatry, 21, 383-390. doi:10.1080/00048678709160935

McLaughlin, K. A., Fairbank, J. A., Gruber, M. J., Jones, R. T., Osofsky, J. D., Pfefferbaum, B., ... \& Kessler, R. C. (2010). Trends in serious emotional disturbance among youths exposed to Hurricane Katrina. Journal of the American Academy of Child \& Adolescent Psychiatry, 49, 9901000. doi.org/10.1016/j.jaac.2010.06.012

McLaughlin, K. A., Fairbank, J. A., Gruber, M. J., Jones, R. T., Lakoma, M. D., Pfefferbaum, B., ... \& Kessler, R. C. (2009). Serious emotional disturbance among youths exposed to Hurricane Katrina 2 years postdisaster. Journal of the American Academy of Child \& Adolescent Psychiatry, 48, 1069-1078.doi:10.1097/CHI.0b013e3181b76697

McLean, M. A., Cobham, V. E., \& Simcock, G. (2018). Prenatal Maternal Distress: A Risk Factor for Child Anxiety? Clinical Child and Family Psychology Review, 21, 203-223. doi:10.1007/s10567017-0251-4

McLean, M. A., Cobham, V. E., Simcock, G., Elgbeili, G., Kildea, S., \& King, S. (2018). The role of prenatal maternal stress in the development of childhood anxiety symptomatology: The QF2011 Queensland Flood Study. Development and Psychopathology, 30, 995-1007. doi:10.1017/s0954579418000408

Mercuri, A., \& Angelique, H. L. (2004). Children's responses to natural, technological, and na-tech disasters. Community Mental Health Journal, 40, 167-175. doi:10.1023/b:comh.0000022735.38750 
Meyer, A., Danielson, C. K., Danzig, A. P., Bhatia, V., Black, S. R., Bromet, E., ... \& Klein, D. N. (2017). Neural biomarker and early temperament predict increased internalizing symptoms after a natural disaster. Journal of the American Academy of Child \& Adolescent Psychiatry, 56, 410-416. doi:10.1016/j.jaac.2017.02.005

Mikolajewski, A. J., \& Scheeringa, M. S. (2018). Examining the Prospective Relationship between Pre-Disaster Respiratory Sinus Arrhythmia and Post-Disaster Posttraumatic Stress Disorder Symptoms in Children. Journal of Abnormal Child Psychology, 46, 15351545.doi:10.1007/s10802-017-0396-0

Mikyung Jang, Se-hwa Lee \& Lee-jin Kim (2020) Post-traumatic stress disorder and behavioral problems of parents and children after the 2015 Nepal earthquakes. International Journal of Mental Health, 49, 3-16, DOI: 10.1080/00207411.2020.1725719

Miller, P. A., Roberts, N. A., Zamora, A. D., Weber, D. J., Burleson, M. H., Robles, E., \& Tinsley, B. J. (2012). Families coping with natural disasters: Lessons from wildfires and tornados. Qualitative Research in Psychology, 9, 314-336. doi:10.1080/14780887.2010.500358

Milne, G. (1977). Cyclone Tracy: II the effects on Darwin children. Australian Psychologist, 12, 5562. doi:10.1080/00050067708255860

Mooney, M., Tarrant, R., Paton, D., Johnston, D., \& Johal, S. (2020). The school community contributes to how children cope effectively with a disaster. Pastoral Care in Education, 1-24. doi:10.1080/02643944.2020.1774632

Moore, K. W., \& Varela, R. E. (2010). Correlates of long-term posttraumatic stress symptoms in children following Hurricane Katrina. Child Psychiatry \& Human Development, 41, 239250.doi:10.1007/s10578-009-0165-6 
RESILIENCE IN CHILDREN AFTER NATURAL DISASTERS

Mordeno, I. G., Galela, D. S., Nalipay, M. J. N., \& Cue, M. P. (2018). Centrality of Event and Mental Health Outcomes in Child and Adolescent Natural Disaster Survivors. The Spanish Journal of Psychology, 21. doi:10.1017/sjp.2018.58

Moss, K. M., Simcock, G., Cobham, V., Kildea, S., Elgbeili, G., Laplante, D. P., \& King, S. (2017). A potential psychological mechanism linking disaster-related prenatal maternal stress with child cognitive and motor development at 16 months: The QF2011 Queensland Flood Study. Developmental Psychology, 53, 629-641. doi:10.1037/dev0000272

Mouchenik, Y., Marty-Chevreuil, A., Marquer, C., Joseph, N. E., Ducasse, J. W., Ryswick, C., ... \& Baubet, T. (2014). Indicative evaluation of psychological disturbance amongst young children affected by the January 2010 Haiti earthquake, in Port-au-Prince. Vulnerable Children and Youth Studies, 9, 247-257. doi:10.1080/17450128.2014.901589

Mumper, E. E., Dyson, M. W., Finsaas, M. C., Olino, T. M., \& Klein, D. N. (2019). Life stress moderates the effects of preschool behavioral inhibition on anxiety in early adolescence. Journal of Child Psychology and Psychiatry, 61, 167-174. doi:10.1111/jcpp.13121

Muris, P., Meesters, C., Merckelbach, H., Verschuren, M., Geebelen, E., \& Aleva, E. (2002). Fear of storms and hurricanes in Antillean and Belgian children. Behaviour Research and Therapy, 40, 459-469. doi:10.1016/S0005-7967(01)00058-4

Murphy, S. A. (2010). Women's and Children's Exposure to Mass Disaster and Terrorist Attacks. Issues in Mental Health Nursing, 31, 45-53. doi:10.3109/01612840903200035

Najarian, L. M., Goenjian, A. K., Pelcovttz, D., Mandel, F., \& Najarian, B. (1996). Relocation after a disaster: Posttraumatic stress disorder in Armenia after the earthquake. Journal of the American 
RESILIENCE IN CHILDREN AFTER NATURAL DISASTERS

Academy of Child \& Adolescent Psychiatry, 35, 374-383. doi:10.1097/00004583-19960300000020

Navarro, J., Pulido, R., Berger, C., Arteaga, M., Osofsky, H. J., Martinez, M., ... \& Hansel, T. C. (2016). Children's disaster experiences and psychological symptoms: An international comparison between the Chilean earthquake and tsunami and Hurricane Katrina. International Social Work, 59, 545-558. doi:10.1177/0020872814537850

Neuner, F., Schauer, E., Catani, C., Ruf, M., \& Elbert, T. (2006). Post-tsunami stress: A study of posttraumatic stress disorder in children living in three severely affected regions in Sri Lanka. Journal of Traumatic Stress: Official Publication of The International Society for Traumatic Stress Studies, 19, 339-347. doi:10.1002/jts.20121

Nomura, Y., Davey, K., Pehme, P. M., Finik, J., Glover, V., Zhang, W., ... Ham, J. (2019). Influence of in utero exposure to maternal depression and natural disaster-related stress on infant temperament at 6 months: The children of Superstorm Sandy. Infant Mental Health Journal, 40, 204-216.doi:10.1002/imhj.21766

Norris, F. H., Friedman, M. J., Watson, P. J., Byrne, C. M., Diaz, E., \& Kaniasty, K. (2002). 60,000 disaster victims speak: Part I. an empirical review of the empirical literature, 1981-2001. Psychiatry: Interpersonal and Biological Processes, 65, 207-239. doi:10.1521/psyc.65.3.207.20173

Nygaard, E., Jensen, T. K., \& Dyb, G. (2010). Posttraumatic stress reactions in siblings after mutual disaster: Relevance of family factors. Journal of Traumatic Stress: Official Publication of The International Society for Traumatic Stress Studies, 23, 278-281. doi:10.1002/jts.20511 
Nygaard, E., Jensen, T. K., \& Dyb, G. (2012). Stability of posttraumatic stress reaction factors and their relation to general mental health problems in children: A longitudinal study. Journal of Clinical Child \& Adolescent Psychology, 41, 15-26. doi:10.1080/15374416.2012.632344

Ortiz, C. D., Silverman, W. K., Jaccard, J., \& La Greca, A. M. (2011). Children's state anxiety in reaction to disaster media cues: A preliminary test of a multivariate model. Psychological Trauma: Theory, Research, Practice, and Policy, 3, 157-164. doi:10.1037/a0020098

Osofsky, H. J., Osofsky, J. D., Kronenberg, M., Brennan, A., \& Hansel, T. C. (2009). Posttraumatic stress symptoms in children after Hurricane Katrina: Predicting the need for mental health services. American Journal of Orthopsychiatry, 79, 212-220. doi.org/10.1037/a0016179

Osofsky, J. D., Osofsky, H. J., Weems, C. F., Hansel, T. C., \& King, L. S. (2014). Effects of stress related to the gulf oil spill on child and adolescent mental health. Journal of Pediatric Psychology, 41, 65-72. doi.org/10.1093/jpepsy/jsu085

Osofsky, J., Kronenberg, M., Bocknek, E., \& Hansel, T. C. (2015, August). Longitudinal impact of attachment-related risk and exposure to trauma among young children after Hurricane Katrina. Child \& Youth Care Forum, 44, 493-510. doi:10.1007/s10566-015-9300-7

Pfefferbaum, B., Newman, E., \& Nelson, S. D. (2014). Mental health interventions for children exposed to disasters and terrorism. Journal of Child and Adolescent Psychopharmacology, 24, 2431. doi:10.1089/cap.2013.0061

Pfefferbaum, B., Nitiéma, P., Tucker, P., \& Newman, E. (2017). Early child disaster mental health interventions: A review of the empirical evidence. Child \& Youth Care Forum, 46, 621-642. doi:10.1007/s10566-017-9397-y 
Pfefferbaum, B., Varma, V., Nitiéma, P., \& Newman, E. (2014). Universal preventive interventions for children in the context of disasters and terrorism. Child and Adolescent Psychiatric Clinics of North America, 23, 363-382. doi:10.1016/j.chc.2013.12.006

Pfefferbaum, B., Weems, C. F., Scott, B. G., Nitiéma, P., Noffsinger, M. A., Pfefferbaum, R. L., ,.. Chakraburtty, A. (2013). Research methods in child disaster studies: A review of studies generated by the september 11, 2001, terrorist attacks; the 2004 Indian Ocean Tsunami; and Hurricane Katrina. Child \& Youth Care Forum, 42, 285-337. doi:10.1007/s10566-013-9211-4

Pina, A. A., Villalta, I. K., Ortiz, C. D., Gottschall, A. C., Costa, N. M., \& Weems, C. F. (2008). Social support, discrimination, and coping as predictors of posttraumatic stress reactions in youth survivors of Hurricane Katrina. Journal of Clinical Child \& Adolescent Psychology, 37, 564-574. doi: $10.1080 / 15374410802148228$

Pine, D. S., \& Cohen, J. A. (2002). Trauma in children and adolescents: Risk and treatment of psychiatric sequelae. Biological Psychiatry, 51, 519-531. doi:10.1016/s0006-3223(01)01352-x

Piotrowski, C., \& Dunham, F. Y. (1983). Locus of control orientation and perception of "hurricane" in fifth graders. The Journal of general psychology, 109, 119-127. doi:10.1080/00221309.1983.9711518

Pityaratstian, N., Piyasil, V., Ketumarn, P., Sitdhiraksa, N., Ularntinon, S., \& Pariwatcharakul, P. (2014). Randomized controlled trial of Group Cognitive Behavioural Therapy for Post-Traumatic 
RESILIENCE IN CHILDREN AFTER NATURAL DISASTERS

Stress Disorder in children and adolescents exposed to tsunami in Thailand. Behavioural and Cognitive Psychotherapy, 43, 549-561. doi:10.1017/s1352465813001197

Ponnamperuma, T., \& Nicolson, N. A. (2016). Negative trauma appraisals and PTSD symptoms in Sri Lankan adolescents. Journal of abnormal child psychology, 44, 245-255. doi.org/10.1007/s10802-015-9985-y

Powell, T. M., \& Bui, T. (2016). Supporting social and emotional skills after a disaster: Findings from a mixed methods study. School Mental Health, 8, 106-119. doi:10.1007/s12310-016-9180-5

Powell, T. M., \& Thompson, S. J. (2016). Enhancing coping and supporting protective factors after a disaster. Research on Social Work Practice, 26, 539-549. doi:10.1177/1049731514559422

Prinstein, M. J., La Greca, A. M., Vernberg, E. M., \& Silverman, W. K. (1996). Children's coping assistance: How parents, teachers, and friends help children cope after a natural disaster. Journal of Clinical Child Psychology, 25, 463-475. doi:10.1207/s15374424jccp2504_11

Proctor, L. J., Fauchier, A., Oliver, P. H., Ramos, M. C., Rios, M. A., \& Margolin, G. (2007). Family context and young children's responses to earthquake. Journal of Child Psychology and Psychiatry, 48, 941-949. doi:10.1111/j.1469-7610.2007.01771.x

Pullins, L. G., McCammon, S. L., Lamson, A. S., Wuensch, K. L., \& Mega, L. (2005). School-based post-flood screening and evaluation: Findings and challenges in one community. Stress, Trauma, and Crisis, 8, 229-249. doi:10.1080/1543461050 0406343

Pynoos, R. S., Goenjian, A., Tashjian, M., Karakashian, M., Manjikian, R., Manoukian, G., ... \& Fairbanks, L. A. (1993). Post-trauatic stress reactions in children after the 1988 Armenian earthquake. The British Journal of Psychiatry, 163, 239-247. doi:10.1192/bjp.163.2.239 


\section{RESILIENCE IN CHILDREN AFTER NATURAL DISASTERS}

Raccanello, D., Burro, R., \& Hall, R. (2017). Children's emotional experience two years after an earthquake: An exploration of knowledge of earthquakes and associated emotions. PLoS one, 12, e0189633.doi: 10.1371/journal.pone.0189633

Roberts, Y. H., Mitchell, M. J., Witman, M., \& Taffaro, C. (2010). Mental health symptoms in youth affected by Hurricane Katrina. Professional Psychology: Research and Practice, 41, 10-18. doi:10.1037/a0018339

Rousseau, C., Benoit, M., Lacroix, L., \& Gauthier, M.-F. (2009). Evaluation of a sandplay program for preschoolers in a multiethnic neighborhood. Journal of Child Psychology and Psychiatry, 50, 743-750. doi:10.1111/j.1469-7610.2008.02003.x

Roussos, A., Goenjian, A. K., Steinberg, A. M., Sotiropoulou, C., Kakaki, M., Kabakos, C., ... \& Manouras, V. (2005). Posttraumatic stress and depressive reactions among children and adolescents after the 1999 earthquake in Ano Liosia, Greece. American Journal of Psychiatry, 162, 530-537. doi: 10.1176/appi.ajp.162.3.530

Roysircar, G., Colvin, K. F., Afolayan, A. G., Thompson, A., \& Robertson, T. W. (2017). Haitian children's resilience and vulnerability assessed with house-tree-person (HTP) drawings. Traumatology, 23, 68. doi:10.1037/trm0000090

Rubens, S. L., Felix, E. D., \& Hambrick, E. P. (2018). A meta-analysis of the impact of natural disasters on internalizing and externalizing problems in youth. Journal of Traumatic Stress, 31, 332-341. doi:10.1002/jts.22292

Russell, J. D., Neill, E. L., Carrión, V. G., \& Weems, C. F. (2017). The network structure of posttraumatic stress symptoms in children and adolescents exposed to disasters. Journal of the 
RESILIENCE IN CHILDREN AFTER NATURAL DISASTERS

American Academy of Child \& Adolescent Psychiatry, 56, 669-677. doi.org/10.1016/j.jaac.2017.05.021

Russoniello, C. V., Skalko, T. K., O'brien, K., McGhee, S. A., Bingham-Alexander, D., \& Beatley, J. (2002). Childhood posttraumatic stress disorder and efforts to cope after Hurricane Floyd. Behavioral Medicine, 28, 61-71. doi:10.1080/08964280209596399

Şahin, N. H., Batıgün, A. D., \& Yılmaz, B. (2007). Psychological symptoms of Turkish children and adolescents after the 1999 earthquake: Exposure, gender, location, and time duration. Journal of Traumatic Stress: Official Publication of The International Society for Traumatic Stress Studies, 20, 335-345. doi.org/10.1002/jts.20217

Sales, J. M., Fivush, R., Parker, J., \& Bahrick, L. (2005). Stressing memory: Long-term relations among children's stress, recall and psychological outcome following hurricane Andrew. Journal of Cognition and Development, 6, 529-545. doi: 10.1207/s15327647jcd0604_5

Salloum, A., \& Lewis, M. L. (2010). An exploratory study of African American parent-child coping strategies post-Hurricane Katrina. Traumatology, 16, 31-41. doi:10.1177/1534765609348240

Salloum, A., \& Overstreet, S. (2008). Evaluation of individual and group grief and trauma interventions for children post disaster. Journal of Clinical Child \& Adolescent Psychology, 37, 495-507. doi:10.1080/15374410802148194

Salloum, A., Carter, P., Burch, B., Garfinkel, A., \& Overstreet, S. (2011). Impact of exposure to community violence, Hurricane Katrina, and Hurricane Gustav on posttraumatic stress and depressive symptoms among school age children. Anxiety, Stress, \& Coping, 24, 27-42. doi:10.1080/10615801003703193

Saulnier, D. D., \& Brolin, K. (2015). A systematic review of the health effects of prenatal exposure to disaster. International Journal of Public Health, 60, 781-787. doi:10.1007/s00038-015-0699-2 
Saylor, C. F., Swenson, C. C., Stokes Reynolds, S., \& Taylor, M. (1999). The Pediatric Emotional Distress Scale: A brief screening measure for young children exposed to traumatic events. Journal of Clinical Child Psychology, 28, 70-81.doi:10.1207/s15374424jccp2801_6

Scaramella, L. V., Sohr-Preston, S. L., Callahan, K. L., \& Mirabile, S. P. (2008). A test of the Family Stress Model on toddler-aged children's adjustment among Hurricane Katrina impacted and nonimpacted low-income families. Journal of Clinical Child \& Adolescent Psychology, 37, 530541. doi:10.1080/15374410802148202

Scheeringa, M. S. (2015, August). Untangling psychiatric comorbidity in young children who experienced single, repeated, or Hurricane Katrina traumatic events. Child \& Youth Care Forum, 44, 475-492. doi:10.1007/s10566-014-9293-7

Scheeringa, M. S., \& Zeanah, C. H. (2008). Reconsideration of harm's way: Onsets and comorbidity patterns of disorders in preschool children and their caregivers following Hurricane Katrina. Journal of Clinical Child \& Adolescent Psychology,37, 508-518. doi:10.1080/15374410802148178

Schwind, J. S., Formby, C. B., Santangelo, S. L., Norman, S. A., Brown, R., Hoffman Frances, R., ... Karmacharya, D. (2018). Earthquake exposures and mental health outcomes in children and adolescents from Phulpingdanda village, Nepal: a cross-sectional study. Child and Adolescent Psychiatry and Mental Health, 12, 54. doi:10.1186/s13034-018-0257-9

Self-Brown, S., Lai, B. S., Harbin, S., \& Kelley, M. L. (2014). Maternal posttraumatic stress disorder symptom trajectories following Hurricane Katrina: An initial examination of the impact of 
RESILIENCE IN CHILDREN AFTER NATURAL DISASTERS

maternal trajectories on the well-being of disaster-exposed youth. International Journal of Public Health, 59, 957-965. doi:10.1007/s00038-014-0596-0

Shannon, M. P., Lonigan, C. J., Finch Jr, A. J., \& Taylor, C. M. (1994). Children exposed to disaster: I. Epidemiology of post-traumatic symptoms and symptom profiles. Journal of the American Academy of Child \& Adolescent Psychiatry, 33, 80-93. doi:10.1097/00004583-199401000-00012

Shaw, J. A., Applegate, B., \& Schorr, C. (1996). Twenty-one-month follow-up study of school-age children exposed to Hurricane Andrew. Journal of the American Academy of Child \& Adolescent Psychiatry, 35, 359-364. doi:10.1097/00004583-199603000-00018.

Shaw, J. A., Applegate, B., Tanner, S., Perez, D., Rothe, E., Campo-Bowen, A. E., \& Lahey, B. L. (1995). Psychological effects of Hurricane Andrew on an elementary school population. Journal of the American Academy of Child \& Adolescent Psychiatry, 34, 1185-1192. doi:10.1097/00004583-199509000-00016

Shen, Y.-J. (2002). Short-term group play therapy with Chinese earthquake victims: Effects on anxiety, depression and adjustment. International Journal of Play Therapy, 11, 43-63. doi:10.1037/h0088856

Silwal, S., Dybdahl, R., Chudal, R., Sourander, A., \& Lien, L. (2018). Psychiatric symptoms experienced by adolescents in Nepal following the 2015 earthquakes. Journal of affective disorders, 234, 239-246. doi:10.1016/j.jad.2018.03.002

Simcock, G., Cobham, V. E., Laplante, D. P., Elgbeili, G., Gruber, R., Kildea, S., \& King, S. (2019). A cross-lagged panel analysis of children's sleep, attention, and mood in a prenatally stressed cohort: The QF2011 Queensland flood study. Journal of Affective Disorders, 255, 96-104. doi:10.1016/j.jad.2019.05.041 
RESILIENCE IN CHILDREN AFTER NATURAL DISASTERS

Simcock, G., Elgbeili, G., Laplante, D. P., Kildea, S., Cobham, V., Stapleton, H., ... King, S. (2017).

The Effects of Prenatal Maternal Stress on Early Temperament. Journal of Developmental \& Behavioral Pediatrics, 38, 310-321. doi:10.1097/dbp.0000000000000444

Simcock, G., Laplante, D. P., Elgbeili, G., Kildea, S., \& King, S. (2018). A trajectory analysis of childhood motor development following stress in pregnancy: The QF2011 flood study. Developmental Psychobiology, 60, 836-848. doi:10.1002/dev.21767

Siqveland, J., Hafstad, G. S., \& Tedeschi, R. G. (2012). Posttraumatic growth in parents after a natural disaster. Journal of Loss and Trauma, 17, 536-544. doi:10.1080/15325024.2012.678778

Siswa Widyatmoko, C., Tan, E. T., Conor Seyle, D., Haksi Mayawati, E., \& Cohen Silver, R. (2011). Coping with natural disasters in Yogyakarta, Indonesia: The psychological state of elementary school children as assessed by their teachers. School Psychology International, 32, 484-497. doi:10.1177/0143034311402919

Soysa, C. K. (2013). War and tsunami PTSD responses in Sri Lankan children: Primacy of reexperiencing and arousal compared to avoidance-numbing. Journal of Aggression, Maltreatment \& Trauma, 22, 896-915. doi:10.1080/10926771.2013.824056

Spell, A. W., Kelley, M. L., Wang, J., Self-Brown, S., Davidson, K. L., Pellegrin, A., ... \& Baumeister, A. (2008). The moderating effects of maternal psychopathology on children's adjustment posthurricane Katrina. Journal of Clinical Child \& Adolescent Psychology, 37, 553-563. doi:10.1080/15374410802148210

Sprung, M. (2008). Unwanted intrusive thoughts and cognitive functioning in kindergarten and young elementary school-age children following Hurricane Katrina. Journal of Clinical Child \& Adolescent Psychology, 37, 575-587. doi:10.1080/15374410802148236 
Sprung, M., \& Harris, P. L. (2010). Intrusive thoughts and young children's knowledge about thinking following a natural disaster. Journal of Child Psychology and Psychiatry, 51, 1115-1124. doi:10.1111/j.14697610.2010.02273.x

Sriskandarajah, V., Neuner, F., \& Catani, C. (2015). Predictors of violence against children in Tamil families in northern Sri Lanka. Social Science \& Medicine, 146, 257-265. doi:10.1016/j.socscimed.2015.10.010

Stoppelbein, L., \& Greening, L. (2000). Posttraumatic stress symptoms in parentally bereaved children and adolescents. Journal of the American Academy of Child \& Adolescent Psychiatry, 39, 1112-1119. doi:10.1097/00004583-200009000-00010

Strahm, A. M., Bagne, A. G., Rued, H. A., Larson, K. J., Roemmich, J. N., \& Hilmert, C. J. (2020). Prenatal traumatic stress and offspring hair cortisol concentration: A nine year follow up to the Red River flood pregnancy study. Psychoneuroendocrinology, 113, 104579. doi:10.1016/j.psyneuen.2019.104579

Sullivan, M. A., Saylor, C. F., \& Foster, K. Y. (1991). Post-hurricane adjustment of preschoolers and their families. Advances in Behaviour Research and Therapy, 13, 163-171. doi:10.1016/01466402(91)90004-T

Sun, X. Y., Fan, H. M., Bai, B., Song, H. T., Tao, F. Y., Song, Z. X., ... \& Zhang, L. Y. (2014). Psychosomatic conditions of the children and adolescents exposed to 5.12 Wenchuan earthquake. International Journal of Behavioral Medicine, 21, 730-735. doi:10.1007/s12529$013-9377-z$ 
Sun, Y., \& Yan, T. (2020). Relationship between household composition and intergenerational postdisaster mental health in two-parent families: Evidence from Japan. Journal of affective disorders, 270, 22-29. doi.org/10.1016/j.jad.2020.03.052

Swenson, C. C., Saylor, C. F., Powell, M. P., Stokes, S. J., Foster, K. Y., \& Belter, R. W. (1996). Impact of a natural disaster on preschool children: Adjustment 14 months after a hurricane. American Journal of Orthopsychiatry, 66, 122-130. doi:10.1037/h0080162

Takada, S. (2013). Post-Traumatic Stress Disorders and mental health care (lessons learned from the Hanshin-Awaji Earthquake, Kobe, 1995). Brain and Development, 35, 214-219. doi:10.1016/j.braindev.2012.09.013

Tang, B., Liu, X., Liu, Y., Xue, C., \& Zhang, L. (2014). A meta-analysis of risk factors for depression in adults and children after natural disasters. Bio Med Central Public Health, 14, 623. doi:10.1186/1471-2458-14-623

Tang, W., Lu, Y., \& Xu, J. (2018). Post-traumatic stress disorder, anxiety and depression symptoms among adolescent earthquake victims: comorbidity and associated sleep-disturbing factors. Social Psychiatry and Psychiatric Epidemiology, 53, 1241-125doi:10.1007/s00127-018-1576-0

Tang, W., Xu, D., Li, B., Lu, Y., \& Xu, J. (2018). The relationship between the frequency of suicidal ideation and sleep disturbance factors among adolescent earthquake victims in China. General Hospital Psychiatry, 55, 90-97. doi:10.1016/j.genhosppsych.2018.09.013

Tang, W., Zhao, J., Lu, Y., Yan, T., Wang, L., Zhang, J., \& Xu, J. (2017). Mental health problems among children and adolescents experiencing two major earthquakes in remote mountainous $\begin{array}{llll}\text { regions: a longitudinal study. Comprehensive } & \text { Psychiatry, 72, 66-73. }\end{array}$ doi:10.1016/j.comppsych.2016.09.004 


\section{RESILIENCE IN CHILDREN AFTER NATURAL DISASTERS}

Tang, W., Zhao, J., Lu, Y., Zha, Y., Liu, H., Sun, Y., ... \& Xu, J. (2018). Suicidality, posttraumatic stress, and depressive reactions after earthquake and maltreatment: a cross-sectional survey of a random sample of 6132 Chinese children and adolescents. Journal of Affective Disorders, 232, 363-369. doi:10.1016/j.jad.2018.02.081

Tao, T., Duan, X., \& Shi, J. (2014). Posttraumatic stress symptoms of Chinese rural children and adolescents surviving the 2008 Wenchuan earthquake assessed using CRIES. Journal of Loss and Trauma, 19, 1-11. doi:10.1080/15325024.2012.734201

Tatsuta, N., Nakai, K., Satoh, H., \& Murata, K. (2015). Impact of the great east Japan earthquake on child's IQ. The Journal of Pediatrics, 167, 745-751. doi:10.1016/j.jpeds.2015.06.033

Tees, M. T., Harville, E. W., Xiong, X., Buekens, P., Pridjian, G., \& Elkind-Hirsch, K. (2010). Hurricane Katrina-related maternal stress, maternal mental health, and early infant temperament. Maternal and Child Health Journal, 14, 511-518. doi:10.1007/s10995-009-0486$\underline{x}$

Terranova, A. M., Boxer, P., \& Morris, A. S. (2009). Factors influencing the course of posttraumatic stress following a natural disaster: Children's reactions to Hurricane Katrina. Journal of Applied Developmental Psychology, 30, 344-355. doi:10.1016/j.appdev.2008.12.017

Terranova, A. M., Morris, A. S., Myers, S., Kithakye, M., \& Morris, M. D. (2015). Preschool children's adjustment following a hurricane: Risk and resilience in the face of adversity. Early Education and Development, 26, 534-548. doi:10.1080/10409289.2015.994463

Thienkrua, W., Cardozo, B. L., Chakkraband, M. S., Guadamuz, T. E., Pengjuntr, W., Tantipiwatanaskul, P., ... \& Tappero, J. W. (2006). Symptoms of posttraumatic stress disorder and depression among children in tsunami-affected areas in southern Thailand. Jama, 296, 549-559. doi:10.1001/jama.296.5.549 
Thomson, J., Seers, K., Frampton, C., Hider, P., \& Moor, S. (2016). Sequential population study of the impact of earthquakes on the emotional and behavioural well-being of 4-year-olds in Canterbury, New Zealand. Journal of Paediatrics and Child Health, 52, 18-24. doi:10.1111/jpc. 12988

Tian W, Jia Z, Duan G, Liu W, Pan X, Guo Q, Chen R, Zhang X. (2013). Longitudinal study on health-related quality of life among child and adolescent survivors of the 2008 Sichuan earthquake. Quality of Life Research: An International Journal of Quality of Life Aspects of Treatment, Care \& Rehabilitation, 22, 745-752.doi:10.1007/s11136-012-0201-z

Tolin, D. F., \& Foa, E. B. (2006). Sex differences in trauma and posttraumatic stress disorder: A quantitative review of 25 years of research. Psychological Bulletin, 132, 959-992. doi:10.1037/0033-2909.132.6.959

Udomratn, P. (2009). Prevalence of tsunami-related PTSD and MDD in Thailand. Asian Journal of Psychiatry, 2, 124-127. doi:10.1016/j.ajp.2009.10.008

Uemoto, M., Asakawa, A., Takamiya, S., Asakawa, K., \& Inui, A. (2012). Kobe earthquake and posttraumatic stress in school-aged children. International Journal of Behavioral Medicine, 19, 243251. doi:10.1007/s12529-011-9184-3

Usami, M., Iwadare, Y., Kodaira, M., Watanabe, K., Aoki, M., Katsumi, C., ... \& Tanaka, H. (2012). Relationships between traumatic symptoms and environmental damage conditions among children 8 months after the 2011 Japan earthquake and tsunami. PLoS One, 7, e50721. doi:10.1371/journal.pone.0050721 
Usami, M., Iwadare, Y., Ushijima, H., Inazaki, K., Tanaka, T., Kodaira, M., ... Saito, K. (2019). Did kindergarteners who experienced the Great East Japan earthquake as infants develop traumatic symptoms? Series of questionnaire-based cross-sectional surveys. Asian Journal of Psychiatry, 44, 38-44. doi:10.1016/j.ajp.2019.07.011

Usami, M., Iwadare, Y., Watanabe, K., Kodaira, M., Ushijima, H., Tanaka, T., ... \& Saito, K. (2014). Decrease in the traumatic symptoms observed in child survivors within three years of the 2011 Japan earthquake and tsunami. PLoS one, 9, e110898. doi:10.1371/journal.pone.0110898

Valenti, M., Ciprietti, T., Di Egidio, C., Gabrielli, M., Masedu, F., Tomassini, A. R., \& Sorge, G. (2012). Adaptive response of children and adolescents with autism to the 2009 earthquake in L'Aquila, Italy. Journal of Autism and Developmental Disorders, 42, 954-960. doi:10.1007/s10803-011-1323-9

Vernberg, E. M., La Greca, A. M., Silverman, W. K., \& Prinstein, M. J. (1996). Prediction of posttraumatic stress symptoms in children after Hurricane Andrew. Journal of Abnormal Psychology, 105, 237-248. doi:10.1037/0021-843X.105.2.237

Vezzali, L., Cadamuro, A., Versari, A., Giovannini, D., \& Trifiletti, E. (2015). Feeling like a group after a natural disaster: Common ingroup identity and relations with outgroup victims among majority and minority young children. British Journal of Social Psychology, 54, 519-538. doi:10.1111/bjso.12091

Vezzali, L., Drury, J., Versari, A., \& Cadamuro, A. (2016). Sharing distress increases helping and contact intentions via social identification and inclusion of the other in the self: Children's prosocial behaviour after an earthquake. Group Processes \& Intergroup Relations, 19, 314-327. doi: $10.1177 / 1368430215590492$ 
Vigil, J. M., Geary, D. C., Granger, D. A., \& Flinn, M. V. (2010). Sex differences in salivary cortisol, alpha-amylase, and psychological functioning following Hurricane Katrina. Child Development, 81, 1228-1240. doi:10.1111/j.1467-8624.2010.01464.x

Vijayakumar, L., Kannan, G. K., \& Daniel, S. J. (2006). Mental health status in children exposed to tsunami. International Review of Psychiatry, 18, 507-513. doi:10.1080/09540260601037581.

Vijayakumar, L., Kannan, G. K., Ganesh Kumar, B., \& Devarajan, P. (2006). Do all children need intervention after exposure to tsunami? International Review of Psychiatry, 18, 515-522. doi:10.1080/09540260601039876

Vogel, J. M., \& Vernberg, E. M. (1993). Part 1: Children's psychological responses to disasters. Journal of Clinical Child Psychology, 22, 464-484. doi:10.1207/s15374424jccp2204_7

Wang, C.-W., Chan, C. L. W., \& Ho, R. T. H. (2013). Prevalence and trajectory of psychopathology among child and adolescent survivors of disasters: A systematic review of epidemiological studies across 1987-2011. Social Psychiatry and Psychiatric Epidemiology, 48, 1697-1720. doi:10.1007/s00127-013-0731-X

Wang, M., Armour, C., Li, X., Dai, X., Zhu, X., \& Yao, S. (2013). The factorial invariance across gender of three well-supported models: Further evidence for a five-factor model of posttraumatic stress disorder. The Journal of Nervous and Mental Disease,201, 145-152. doi:10.1097/NMD.0b013e31827f627d.

Wang, W., Fu, W., Wu, J., Ma, X. C., Sun, X. L., Huang, Y., ... \& Gao, C. G. (2012). Prevalence of PTSD and depression among junior middle school students in a rural town far from the epicenter of the Wenchuan earthquake in China. PLoS One, 7, e41665. doi:10.1371/journal.pone.0041665 
Ward, M. E., Shelley, K., Kaase, K., \& Pane, J. F. (2008). Hurricane Katrina: A longitudinal study of the achievement and behavior of displaced students. Journal of Education for Students Placed at Risk, 13(2-3), 297-317. doi:10.1080/10824660802350391

Wasserstein, S. B., \& La Greca, A. M. (1998). Hurricane Andrew: Parent conflict as a moderator of children's adjustment. Hispanic Journal of Behavioral Sciences, 20, 212-224. doi: $10.1177 / 07399863980202005$

Weems, C. F., Pina, A. A., Costa, N. M., Watts, S. E., Taylor, L. K., \& Cannon, M. F. (2007). Predisaster trait anxiety and negative affect predict posttraumatic stress in youths after Hurricane Katrina. Journal of Consulting and Clinical Psychology, 75, 154-159. doi:10.1037/0022006X.75.1.154

Weems, C. F., Russell, J. D., Graham, R. A., Neill, E. L., \& Banks, D. M. (2014). Developmental differences in the linkages between anxiety control beliefs and posttraumatic stress in youth. Depression and Anxiety, 32, 356-363. doi:10.1002/da.22319

Weems, C. F., Scott, B. G., Banks, D. M., \& Graham, R. A. (2012). Is TV traumatic for all youths? The role of preexisting posttraumatic-stress symptoms in the link between disaster coverage and stress. Psychological Science, 23, 1293-1297. doi:10.1177/0956797612446952

Weems, C. F., Scott, B. G., Taylor, L. K., Cannon, M. F., Romano, D. M., \& Perry, A. M. (2013). A theoretical model of continuity in anxiety and links to academic achievement in disaster-exposed school children. Development and Psychopathology, 25, 729-737. doi: $10.1017 / \mathrm{S} 0954579413000138$ 
RESILIENCE IN CHILDREN AFTER NATURAL DISASTERS

Weems, C. F., Taylor, L. K., Cannon, M. F., Marino, R. C., Romano, D. M., Scott, B. G., ... \& Triplett, V. (2010). Post traumatic stress, context, and the lingering effects of the Hurricane Katrina disaster among ethnic minority youth. Journal of Abnormal Child Psychology,38, 49-56. doi:10.1007/s10802-009-9352-y

Wolmer, L., Laor, N., Dedeoglu, C., Siev, J., \& Yazgan, Y. (2005). Teacher-mediated intervention after disaster: A controlled three-year follow-up of children's functioning. Journal of Child Psychology and Psychiatry, 46, 1161-1168. doi:10.1111/j.1469-7610.2005.00416.x

Xie, Y., Wu, J., \& Shen, G. (2019). Posttraumatic Growth in Tibetan Adolescent Survivors 6 Years After the 2010 Yushu Earthquake: Depression and PTSD as Predictors. Child Psychiatry \& Human Development, 51, 94-103doi:10.1007/s10578-019-00913-5

Xu, J., Xie, L., Li, B., Li, N., \& Yang, Y. (2012). Anxiety symptoms among children after the Wenchuan earthquake in China. Nordic Journal of Psychiatry,66, 349-354. doi:10.3109/08039488.2011.650197

Yagi, J., Fujiwara, T., Yambe, T., Okuyama, M., Kawachi, I., \& Sakai, A. (2016). Does social capital reduce child behavior problems? Results from the Great East Japan Earthquake follow-up for Children Study. Social Psychiatry and Psychiatric Epidemiology, 51, 1117-1123. doi:10.1007/s00127-016-1227-2

Yang, R., Xiang, Y. T., Shuai, L., Qian, Y., Lai, K. Y., Ungvari, G. S., ... \& Wang, Y. F. (2014). Executive function in children and adolescents with posttraumatic stress disorder 4 and 12 months after the Sichuan earthquake in China. Journal of Child Psychology and Psychiatry, 55, 31-38. doi:10.1111/jcpp.12089 
Yeung, N. C., Lau, J. T., Yu, N. X., Zhang, J., Xu, Z., Choi, K. C., ... \& Lui, W. W. (2016). Media exposure related to the 2008 Sichuan Earthquake predicted probable PTSD among Chinese adolescents in Kunming, China: A longitudinal study, Jurnal of Psychological Trauma, 2, 253262. doi: $10.1037 / \operatorname{tra} 0000121$

Ying, L. H., Wu, X. C., \& Chen, C. (2013). Prevalence and predictors of posttraumatic stress disorder and depressive symptoms among child survivors 1 year following the Wenchuan earthquake in China. European Child \& Adolescent Psychiatry, 22, 567-575. doi:10.1007/s00787-013-0400-3

Ying, L., Wu, X., Lin, C., \& Jiang, L. (2014). Traumatic severity and trait resilience as predictors of posttraumatic stress disorder and depressive symptoms among adolescent survivors of the Wenchuan earthquake. PLoS One, 9, e89401. doi:10.1371/journal.pone.0089401

Yorbik, O., Akbiyik, D. I., Kirmizigul, P., \& Söhmen, T. (2004). Post-traumatic stress disorder symptoms in children after the 1999 Marmara earthquake in Turkey. International Journal of Mental Health, 33, 46-58. doi:10.1080/00207411.2004.11043360

Yoshida, H., Kobayashi, N., Honda, N., Matsuoka, H., Yamaguchi, T., Homma, H., \& Tomita, H. (2016). Post-traumatic growth of children affected by the Great East Japan Earthquake and their attitudes to memorial services and media coverage. Psychiatry and Clinical Neurosciences, 70 , 193-201. doi:10.1111/pcn.12379

Zhang, J., Zhu, S., Du, C., \& Zhang, Y. (2015). Posttraumatic stress disorder and somatic symptoms among child and adolescent survivors following the Lushan earthquake in China: A six-month longitudinal study. Journal of Psychosomatic Research, 79, 100-106. doi:10.1016/j.jpsychores.2015.06.001

Zhang, W., Rajendran, K., Ham, J., Finik, J., Buthmann, J., Davey, K., ... \& Nomura, Y. (2018). Prenatal exposure to disaster-related traumatic stress and developmental trajectories of 
RESILIENCE IN CHILDREN AFTER NATURAL DISASTERS

temperament in early childhood: Superstorm Sandy pregnancy study. Journal of Affective Disorders, 234, 335-345. doi:10.1016/j.jad.2018.02.067

Zhang, X., Liu, M., Zhu, M., Shi, J., \& Cheng, L. (2010). Personality predictors of posttraumatic stress disorder in orphaned survivors of the Sichuan earthquake. Social Behavior and Personality: An International Journal, 38, 1057-1060. doi:10.2224/sbp.2010.38.8.1057

Zhang, Y., Kong, F., Wang, L., Chen, H., Gao, X., Tan, X., ... \& Liu, Y. (2010). Mental health and coping styles of children and adolescent survivors one year after the 2008 Chinese earthquake. Children and Youth Services Review, 32, 1403-1409. doi:10.1016/j.childyouth.2010.06.009

Zhang, Y., Zhang, J., Zhu, S., Du, C., \& Zhang, W. (2015). Prevalence and predictors of somatic symptoms among child and adolescents with probable posttraumatic stress disorder: A crosssectional study conducted in 21 primary and secondary schools after an earthquake. PloS one, 10, e0137101. doi:10.1371/journal.pone.0137101

Zhou, P., Zhang, Y., Wei, C., Liu, Z., \& Hannak, W. (2016). Acute stress disorder as a predictor of posttraumatic stress: A longitudinal study of Chinese children exposed to the Lushan earthquake. PsyCh Journal, 5, 206-214. doi:10.1002/pchj.136

Zhou, X., \& Wu, X. (2016). The relationship between rumination, posttraumatic stress disorder, and posttraumatic growth among Chinese adolescents after earthquake: A longitudinal study. Journal of Affective Disorders, 193, 242-248. doi:10.1016/j.jad.2015.12.076

Zhou, X., Wu, X., \& Zhen, R. (2017). Understanding the relationship between social support and posttraumatic stress disorder/posttraumatic growth among adolescents after Ya'an earthquake: The role of emotion regulation. Psychological Trauma: Theory, Research, Practice, and Policy, 9, 214-221. doi:10.1037/tra0000213 
Zhou, X., Wu, X., \& Zhen, R. (2018). Patterns of Posttraumatic Stress Disorder and Posttraumatic Growth Among Adolescents After the Wenchuan Earthquake in China: A Latent Profile Analysis. Journal of Traumatic Stress, 31, 57-63. doi:10.1002/jts.22246

Zhou, X., Wu, X., Wang, W., \& Tian, Y. (2017). Longitudinal linkages between social support, posttraumatic stress disorder, and posttraumatic growth among primary school students after the Ya'an earthquake. Acta Psychologica Sinica, 49, 1428-1438. doi:10.3724/SP.J.1041.2017.01428

Zhou, X., Wu, X., Zhen, R., Wang, W., \& Tian, Y. (2018). Trajectories of posttraumatic stress disorders among adolescents in the area worst-hit by the Wenchuan earthquake. Journal of Affective Disorders, 235, 303-307. doi:10.1016/j.jad.2018.04.032.

Zhou, X., Zhen, R., \& Wu, X. (2017). Posttraumatic stress disorder symptom severity and control beliefs as the predictors of academic burnout amongst adolescents following the Wenchuan Earthquake. European Journal of Psychotraumatology, 8, 1412227. doi:10.1080/20008198.2017.1412227 